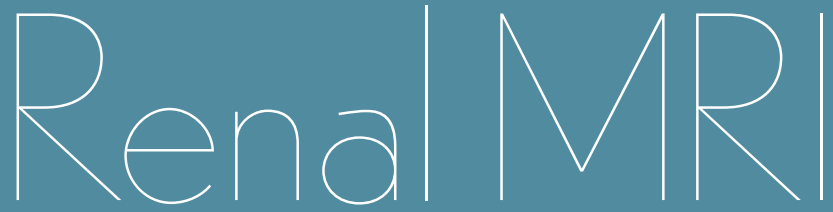

hh Lrom Niche Application to New Tool in Nephrology
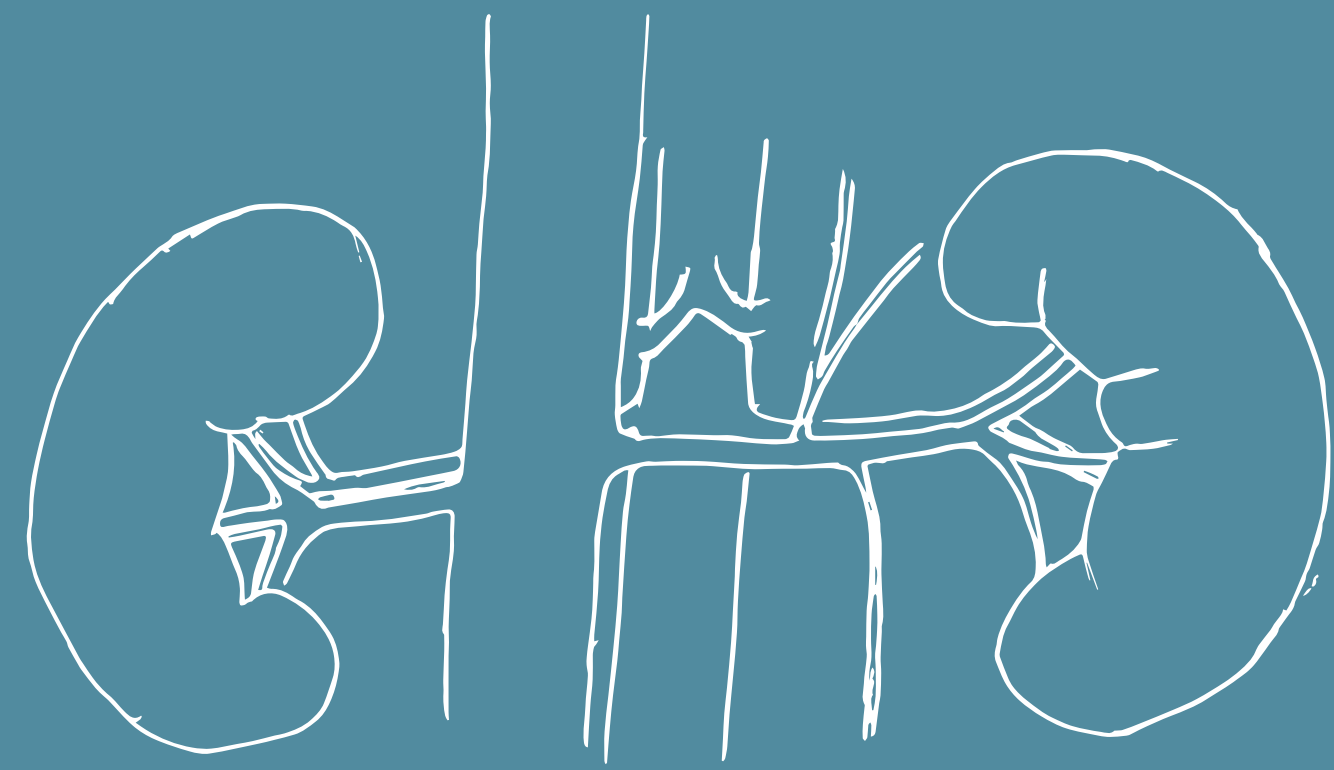

$$
\frac{d}{d t}\left(\begin{array}{l}
M_{x} \\
M_{y} \\
M_{2}
\end{array}\right)=\left(\begin{array}{ccc}
-\frac{1}{T_{2}} & \gamma B_{2} & -\gamma B_{y} \\
-\gamma B_{2} & -\frac{1}{T_{2}} & \gamma B_{x} \\
\gamma B_{y} & -\gamma B_{x} & -\frac{1}{T_{1}}
\end{array}\right)\left(\begin{array}{c}
M_{x} \\
M_{y} \\
M_{a}
\end{array}\right)+\left(\begin{array}{c}
0 \\
0 \\
\frac{M_{0}}{T_{1}}
\end{array}\right)
$$

Anneloes de Boer 



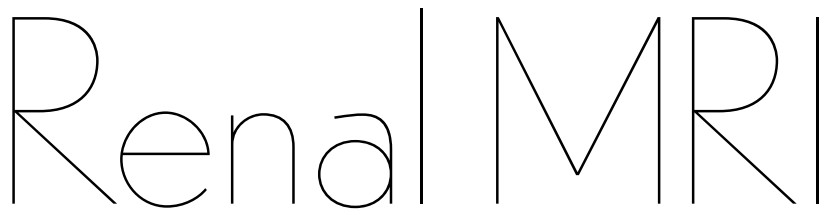

from Niche Application to New Tool in Nephrology

Anneloes de Boer 
Renal MRI - from Niche Application to New Tool in Nephrology

ISBN:

978-94-6423-020-8

Cover design:

Pim Jasper van den Berg

Layout:

Dennis Hendriks, ProefschriftMaken.nl

Printed:

ProefschriftMaken.nl

No part of this publication may be reproduced or transmitted in any form or by means, electronic or mechanical, including photocopy, recording or any other information storage or retrieval system without the prior written permission of the author.

All rights reserved.

Copyright (C) 2020 Anneloes de Boer 


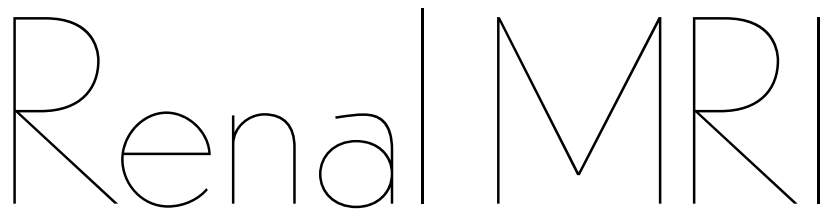

\title{
from Niche Application to New Tool in Nephrology
}

Nier MRI

van Niche tot Nieuw Diagnosticum binnen de Nefrologie (met een samenvatting in het Nederlands)

\section{Proefschrift}

ter verkrijging van de graad van doctor aan de Universiteit Utrecht op gezag van de rector magnificus, prof.dr. H.R.B.M. Kummeling, ingevolge het besluit van het college voor promoties in het openbaar te verdedigen op

woensdag 25 november 2020 des morgens te 11.00 uur

\author{
door \\ Anneloes de Boer \\ geboren op 26 oktober 1990 \\ te Papendrecht
}


Promotoren:

Prof. dr. T. Leiner

Prof. dr. M.C. Verhaar

Copromotoren: Dr. ir. J.M. Hoogduin

Dr. P.J. Blankestijn 


\section{Table of contents}

$\begin{array}{ll}\text { Preface } & 7\end{array}$

$\begin{array}{lll}\text { Chapter } 1 \text { Introduction } & 11\end{array}$

Part I Technical Development 31

Chapter 2 Multi-Parametric Renal MRI - an Intra-Subject 33

Test-Retest Repeatability Study

Chapter 3 Modified Dixon Based Renal Dynamic Contrast Enhanced 69 MRI Facilitates Automated Registration and Perfusion Analysis

Part II Clinical Applications $\quad 95$

Chapter 4 Decreased Native Renal $\mathrm{T}_{1}$ up to One Week after 97

Chapter 5 The Effect of Renal Denervation on Kidney Oxygenation 117 as Determined by BOLD MRI in Patients With Hypertension

Chapter 6 Renal BOLD MRI Relates to Kidney Function and 135 Activity of the Renin-Angiotensin-Aldosterone System in Hypertensive Patients

$\begin{array}{lll}\text { Chapter } 7 & \text { Validation of Multiparametric MRI by Histopathology } & 153\end{array}$ after Nephrectomy: a Case Study

$\begin{array}{lll}\text { Part III Outlook } & 173\end{array}$

Chapter 87 T Renal MRI: Challenges and Promises $\quad 175$

$\begin{array}{lll}\text { Chapter } 9 & \text { Discussion and Conclusion } & 201\end{array}$

$\begin{array}{lll}\text { Appendices Nederlandse Samenvatting } & 219\end{array}$

(English) Summary 223

List of Publications and Presentations $\quad 226$

Dankwoord (Acknowledgements) 230

Curriculum Vitae $\quad 234$ 



\section{Preface}

A PubMed search on "MRI" results in 590,801 hits, and if we refine our search to "brain AND MRI" we find out that 233,623 of those papers (almost half of all papers on MRI) are about the brain. In comparison, a search for "kidney AND MRI" leads to 12,522 results, which is around $2 \%$ of all papers on MRI. Actually, this is a nice reflection of the high-field group in which I spent the last three-and-a-bit years of my life. This group consists of around 5o people, of whom 1.1 (me and my supervisor for $10 \%$ of his time) was doing research on kidney MRI, and almost half were mainly interested in the brain.

Since both kidneys together weigh around 200-300g, only 0.3-0.4\% of body weight, you might argue that the kidneys are overrepresented in MRI research. ${ }^{(1)}$ Following the same argument, the brain would be even more overrepresented since a brain weighs $1300-1400 \mathrm{~g}$, around $2 \%$ of body weight. ${ }^{(1)}$ However, the kidney receives around $17-19 \%$ of cardiac output (the amount of blood exiting the heart per unit of time) while the brain only receives $\sim 12 \%,{ }^{(2)}$ so in terms of cardiac output the kidneys would deserve a little more attention from MR researchers.

If you are a nephrologist, you might think: no wonder that only $2 \%$ of MR papers are about kidneys, whoever makes an MRI of a kidney? In the current clinical practice there is only one direct indication for a kidney MRI apart from assessment of malignancies, which is an MRI of the renal arteries. Another indication might be a contraindication for CT, in which case an MRI is used to detect anatomical deviations.

MR researchers, especially those interested in brain, may wonder: why bother, kidneys move and they are close to bowels with air bubbles inside, so the magnetic field is not homogeneous, and the kidneys are not that interesting anyway, so save yourself the effort... But you are wrong! Kidneys are interesting!

And indeed, kidneys move. A lot. And the magnetic field is inhomogeneous. Just call it a challenge.

However, kidney research is worth the effort - just imagine someone without kidneys: in a few days she ends up in a nephrotoxic coma. And, more importantly, more than $10 \%$ of the general population suffers from some form of kidney disease! (3)

Apart from being very common, kidney disease is a silent killer. Symptoms in the early phase are limited (high blood pressure, aka hypertension) or absent. By the time the patient visits a doctor with vague symptoms like fatigue, the kidney damage is usually in an advanced stage. And kidney function which is lost, cannot be recovered other than with a kidney transplantation.

On the other hand, there is a window of opportunity to act. A large group of patients is known to be at increased risk of kidney disease: people with diabetes 
or hypertension. Therefore, renal function of those patients is generally monitored by their physician, by measuring creatinine in blood and protein in urine in order to timely initiate treatment. Unfortunately, those tests only become abnormal by the time a significant part of renal tissue is already irreversibly damaged. To make optimal use of this window of opportunity, we have to detect renal damage earlier.

In a time of wireless brain sensors, artificial intelligence, CRISPR-cas9, submillimeter mapping of brain activity and so on, the creatinine and urinary albumin-creatinine ratio seem a bit out of date. And yet, it is the best we have. It is good, and cheap, but late. Since we only can slow down progression, since we cannot stop or even reverse it, late is bad. We have to prevent patients ending up at dialysis or with a kidney transplant, and to do that, we have to act in time.

Multiparametric renal MRI can help with this problem. Using MRI, early changes in renal hemodynamics ${ }^{(4)}$ and microstructure ${ }^{(5)}$ can be detected. In general, renal function decline is accompanied by defects in perfusion and oxygenation, which can be detected by arterial spin labelling (ASL) or dynamic contrast enhanced (DCE) MRI and blood oxygenation level dependent (BOLD) MRI, respectively. Alterations in tissue microstructure might be detected with mapping of magnetic properties like $\mathrm{T}_{1}, \mathrm{~T}_{2}$ and diffusion MRI. In this thesis, all those techniques are evaluated. 


\section{References}

1. Molina DK, DiMaio VJ. Normal organ weights in men: part II-the brain, lungs, liver, spleen, and kidneys. Am J Forensic Med Pathol. 2012;33(4):368-72.

2. Williams LR, Leggett RW. Reference values for resting blood flow to organs of man. Clin Phys Physiol Meas. 1989;10(3):187-217.

3. Hill NR, Fatoba ST, Oke JL, Hirst JA, O'Callaghan CA, Lasserson DS, et al. Global Prevalence of Chronic Kidney Disease - A Systematic Review and Meta-Analysis. PLoS One. 2016;11(7):e0158765.

4. Pruijm M, Hofmann L, Piskunowicz M, Muller ME, Zweiacker C, Bassi I, et al. Determinants of renal tissue oxygenation as measured with BOLD-MRI in chronic kidney disease and hypertension in humans. PLoS One. 2014;9(4):e95895.

5. $\quad$ Feng YZ, Chen XQ, Yu J, Liu XL, Cheng ZY, Ren WW, et al. Intravoxel incoherent motion (IVIM) at 3.0 T: evaluation of early renal function changes in type 2 diabetic patients. Abdom Radiol (NY). 2018;43(10):2764-73. 



\section{Chapter 1}

Introduction

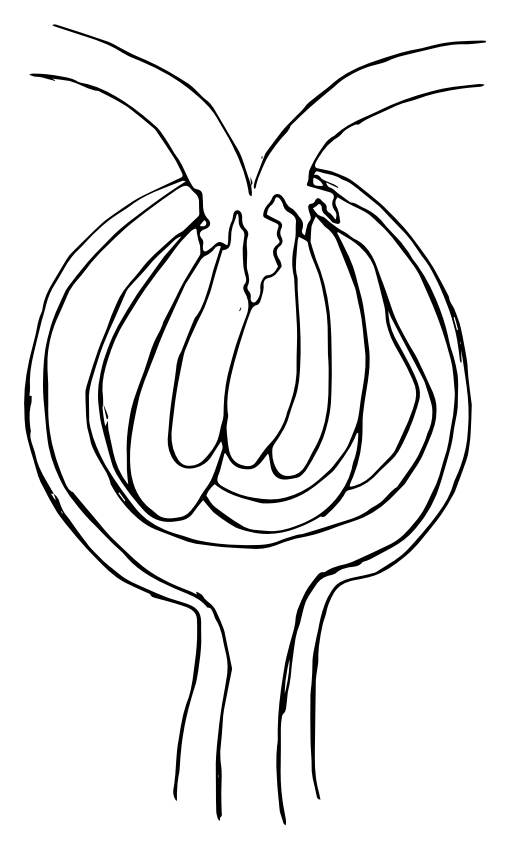




\section{Outline of this Thesis}

The first part of this thesis is focused on the technical development of renal MRI protocols. Chapter 2 describes the results of a repeatability study where a group of healthy volunteers were scanned twice with a comprehensive, multiparametric kidney MRI protocol to assess variability between the scans and to obtain normative values for this protocol. Chapter 3 focuses on a general problem in renal imaging: handling of respiratory motion. In techniques like $\mathrm{T}_{1}$ mapping and DCE imaging contrast changes within the kidney are pronounced, which complicates post-hoc motion correction. In chapter 3 a potential solution for this problem is described. The second part of the thesis focuses on how renal MRI can be used clinically. In chapter 4, a chance finding is described: in the repeatability study (chapter 2) we found residual MR contrast agent up to a week after the scan, a heretofore unknown phenomenon. Chapter 5 and 6 focus on BOLD MRI in hypertensive patients. Chapter 7 describes the multiparametric MRI evaluation of a kidney transplant which was explanted shortly after the scan, which gave us the opportunity to compare the MRI results with pathology. In the last chapter, the opportunities and challenges of renal MRI at 7 Tesla are discussed.

Since this thesis might attract readers from different disciplines, the remainder of this chapter is meant to provide a short introduction to the unknown: for MR physicists to the kidney and for physicians/nephrologists to the wondrous world of MR imaging. ${ }^{1}$

\section{Nephrology for Physicists}

\section{Clinical Practice and Potential of Renal Functional MRI}

(from: "7T Renal MRI: Challenges and Promises" (1))

Currently, diagnostic possibilities in nephrology are limited. Blood plasma creatinine concentration is the most commonly used test to assess renal function. However, at best this offers a rough estimate of glomerular filtration rate (GFR, the clinical measure of renal function) of both kidneys combined. Comparison of plasma and urine osmolality provides some information on renal concentrating capability. In addition, the presence of erythrocytes, protein, and glucose in urine provide information on renal pathology. To obtain detailed information on renal disease, a renal biopsy is required. However, this invasive procedure occasionally leads to major hemorrhagic complications ranging from transient hematuria, occurring in 1-10\% of the cases, to requirement of blood transfusion or surgery in $0.3-7.4$ and

$1 \quad$ The grey parts are for anyone who likes to go a little deeper into the physics. You might want to skip them and save them for when a specific technique crosses your path. 
$0.2-0.5 \%$ of the cases, respectively. ${ }^{(2)}$ Furthermore, there is the risk of sampling error: the tiny bit of tissue that is obtained might not be representative for the entire kidney. Imaging is used when renal artery stenosis or renal masses are suspected, and is limited to anatomical imaging or angiography. ${ }^{(3,4)}$ Functional renal MRI offers the opportunity of expanding the diagnostic possibilities in renal disease, and potentially may decrease the need for renal biopsy.

\section{Renal Anatomy and Physiology}

(from: "7T renal MRI: Challenges and Promises" (1))

The kidneys measure approximately $10-11 \mathrm{~cm}$ in the craniocaudal direction and about $4 \mathrm{~cm}$ in the anteroposterior direction, but renal size strongly depends on body size. ${ }^{(5,6)}$ They consist of an inner medullary part and a superficial renal cortex, ${ }^{(5)}$ with a thickness of about $1 \mathrm{~cm}$ (Figure 1a). ${ }^{(7)}$
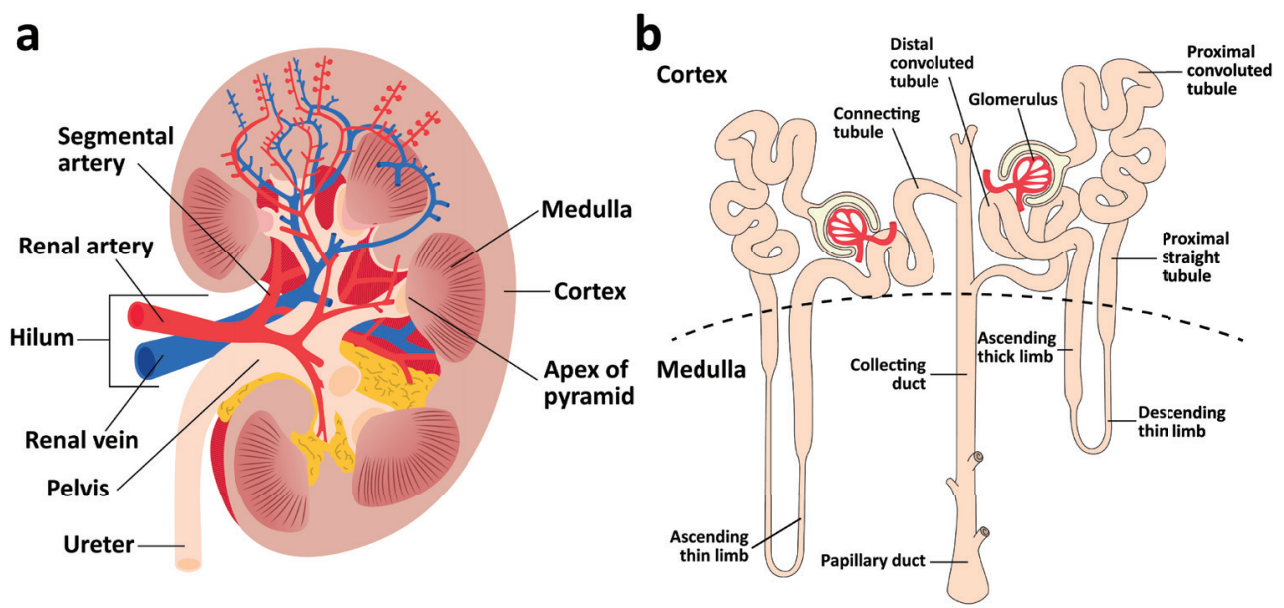

In the cortex, the glomeruli are located, where arterial blood is filtered and pre-urine is created. This pre-urine travels through the proximal tubules to the medulla, and will return to the cortex later on in the distal tubules. The renal medulla consists of the renal pyramids, the apices of which point towards the renal hilum. ${ }^{(5)}$ The pyramids also contain tubules, which empty into the calyces surrounding the apices of the pyramids. The calyces merge into the renal pelvis, from which the ureter originates. Through the hilum run the ureter and the renal artery and vein. The functional unit of the kidney is the nephron, which itself consists of a glomerulus, the proximal convoluted tubule, the loop of Henle and the distal convoluted tubule (Figure 1b). Ultimately, collecting ducts lead the urine to the renal pelvis (Figure $1 \mathrm{~b}$ ). ${ }^{(8)}$

The renal artery enters the renal hilum between the renal pelvis and the renal vein, the renal pelvis lying posteriorly. ${ }^{(9)}$ Thereafter, the artery divides in multiple 
branches: usually one posterior and four anterior segmental arteries. Branches of the segmental renal arteries run in between the medullary pyramids to the cortex. There, they give rise to the afferent arteries supplying the glomeruli. ${ }^{(8)}$ The efferent arteries leaving the glomeruli supply two vascular networks: cortical peritubular capillaries and the vasa recta which descend into the medulla. The descending and ascending vasa recta are closely bundled, which allows the exchange of solutes-for example, oxygen.

Renal filtration occurs in the glomeruli. ${ }^{(8)}$ The filtrate flows through the tubules, where ions, e.g., sodium, are reabsorbed and other substances are secreted into the urine. Due to the renal concentrating mechanism, solutes-mostly sodium and chloride ions-are trapped inside the medulla, resulting in an increase in sodium concentration towards the medulla. Changes in renal tissue sodium concentration potentially yield valuable information on renal function.

Sodium reabsorption occurs in almost all parts of the nephron, but mainly in the medulla. ${ }^{(8,10)}$ Here, in the thick ascending limb of Henle's loop, it is an active process, which largely accounts for the high medullary oxygen demand. However, oxygen delivery is limited in the medulla, both due to limited perfusion (medullary perfusion is about one-fifth of cortical perfusion) and due to the abovementioned counter-current exchange of oxygen in favor of the ascending vasa recta. This leaves the medulla in hypoxic conditions, susceptible to hypoxic damage. ${ }^{(10)}$ Medullary oxygenation can be increased by pharmacological inhibition of oxygen-demanding processes such as sodium reabsorption. An example is furosemide, which inhibits the reabsorption of sodium in the thick ascending limb of the loop of Henle, an effect that can be measured with BOLD MRI. ${ }^{(11)}$

\section{MRI for Physicians}

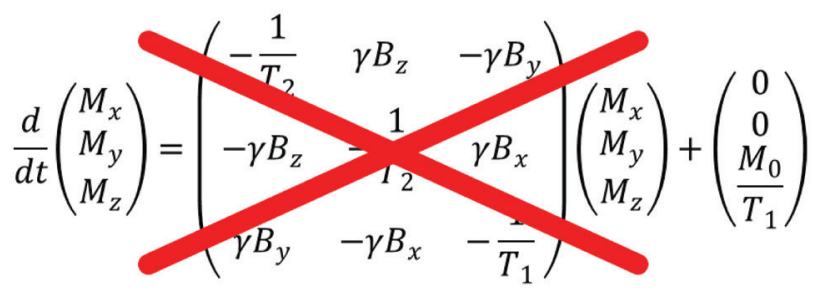

You do not have to understand the physical model of MRI, as represented by the Bloch equation above, to be able to appreciate its capabilities and limitations (hence the red cross). MRI is all about magnetization, as the name already implies (Magnetic Resonance Imaging). And of course, resonance is also important. In the Bloch equation you will notice a few returning variables: the $\mathrm{M}, \mathrm{B}$ and the $\mathrm{T}$ (and $\gamma$, which has to do with resonance). The $\mathrm{M}$ stands for magnetization and the $\mathrm{B}$ 
for magnetic field. ${ }^{(12) 2}$ The subscripts just indicate the direction. However: the $\mathrm{T}$ is important, it stands for an important magnetic property of, in our case, tissue. Here, the subscripts are important since there are two: poetically named $\mathrm{T}_{1}$ and $\mathrm{T}_{2}$.

\section{T, as a Measure of Tissue Fibrosis}

The MR scanner essentially is a big cylindrical magnet - generating a magnetic field. As soon as someone (the patient) is put into this magnetic field he is magnetized, a bit like iron when you put it near a magnet. Iron will behave as a magnet when it is magnetized, attracting other pieces of iron. Something similar happens to the human body: as soon as it is placed inside a magnetic field, it will become magnetized,

creating a second magnetic field (apart from the static field of the scanner). Imagine that the human body is composed of millions of thousands tiny magnets which have the peculiar property that they can either point up or down - anything in between is impossible. Without magnetic field, half of them will point up and the other half down, but when placed in a magnetic field, one of the two (up or down) becomes dominant: the spins are magnetized. The stronger the magnetic field, the more tiny magnets point in the same direction, the stronger the magnetization. So - unless the magnetic field is very strong - not all tiny magnets will point in the same direction. There will be a certain equilibrium with, for example, some pointing down and most pointing up. If we now turn some of those tiny magnets around and release them, they would start to return to the equilibrium. How quickly would they arrive there? Here the time-constants come into play: the $\mathrm{T}_{1}$ defines how long it takes for the magnetization to return to $63 \%$ of its original value. In human kidneys, $\mathrm{T}_{1}$ ranges from 827 and $1676 \mathrm{~ms}$ with lower values at $1.5 \mathrm{~T}$ and higher values at $3 \mathrm{~T},{ }^{(13)}$ let's say 1.5 seconds for now. If the original magnetization is 1 , and we turn just enough magnets around to make sure that half of them point up and the other half down a magnetization of o because they all cancel out - it would take 1.5 seconds for the magnetization to return to 0.63 . And this we can measure.

$\mathrm{T}_{1}$ is an actual, physical property of tissue. For kidneys, the value is between 827 and $1676 \mathrm{~ms}$ with lower values at $1.5 \mathrm{~T}$ and higher values at $3 \mathrm{~T} .{ }^{(13)} \mathrm{In}$ the cortex it is smaller than in the medulla, but this difference disappears in chronic kidney disease. From histology we know that tissue differentiation disappears in chronic kidney disease: ultimately, only fibrosis is left. Apparently, $\mathrm{T}_{1}$ is sensitive to tissue microstructure - in a number of papers it is used as a marker of fibrosis. ${ }^{(13)}$ That

2 In case you wonder why the magnetic field is abbreviated to "B": Maxwell, who first described the magnetic field equations named his variables in alphabetical order, and the magnetic field happened to be second 
makes it a potentially useful marker of disease. However: $\mathrm{T}_{1}$ is very sensitive to a lot of things. Consider it the C-reactive protein of MRI: it is very sensitive but it is not very specific. It depends on the strength of the magnetic field as generated by the scanner: typically this is 1.5 or 3.0 Tesla ( $\mathrm{T}$, the unit to measure magnetic field strength), but there are also $7 \mathrm{~T}$ scanners. In response to oxygen, it shortens. It increases with increasing fluid content. It changes depending on temperature. All those interactions result in fluctuations of $\mathrm{T}_{1}$, from patient to patient, scanner to scanner, time to time. So if we state that $\mathrm{T}_{1}$ is sensitive to microstructure, to tissue fibrosis even, this is not a perfect representation of the truth. But it might very well be good enough - keeping in mind that it is a simplification.

\section{$T_{2}, T_{2}^{*}$ and the BOLD Effect to Measure Oxygenation}

If $\mathrm{T}_{1}$ is about the magnetization in the direction of the main (scanner) magnetic field, $\mathrm{T}_{2}$ has to do with magnetization perpendicular to the main magnetic field. Imagine a bunch of those child's spinning tops? They spin around their axis, but also make a slower swinging motion called "precessing” in physics terms. That's exactly what the millions of thousands tiny magnets in the human body do when they are placed in a magnetic field. When all those magnets precess together, in a synchronized way, we say that the transverse magnetization is maximal. But if some are made to precess a little faster, for example due to a difference in local magnetic field, they are not synchronized anymore and the transverse magnetization decreases. In reality, if someone is placed inside the MR scanner, those magnets never precess in synch and no transverse magnetization is measured. But if we the net magnetization of all magnets by an angle of 90 degrees to the right, you can imagine that all magnets do precess in synch for a while. But some tiny magnets will precess a bit faster and others a bit slower, and therefore after a while the synchronization disappears: the transverse magnetization disappeared. How quickly this happens, is described by the $\mathrm{T}_{2}$ relaxation time constant.

$\mathrm{T}_{2}$ is typically a few hundred milliseconds. For the kidney, it ranges from 76 to $143 \mathrm{~ms}$ at 1.5 and $3 \mathrm{~T}$ (actually that is pretty long compared to other organs). ${ }^{(13)}$ Unlike $\mathrm{T}_{1}, \mathrm{~T}_{2}$ does not depend very heavily on field-strength. Like $\mathrm{T}_{1}$, it is influenced by a lot of parameters, but $\mathrm{T}_{2}$ is especially influenced by the water content of tissue. Apparently, water makes that the tiny magnets remain in synch longer, so water increases $\mathrm{T}_{2}$. This property makes renal $\mathrm{T}_{2}$ a very interesting marker for polycystic kidney disease. You can imagine that within the cysts, $\mathrm{T}_{2}$ is very long - but it appears that $\mathrm{T}_{2}$ is already increased in kidneys with autosomal dominant polycystic kidney disease even before the kidney is enlarged. ${ }^{(14)}$

$\mathrm{T}_{2}$ might also be a relevant measure for other renal diseases. In clinical practice it is important to distinguish inflammation from fibrosis. A lot of MR markers react 
exactly the same to inflammation and to fibrosis ( $\mathrm{T}_{1}$ does, and the apparent diffusion coefficient as measured with diffusion weighted imaging which will be discussed later also), so that is not very helpful for diagnostic purposes (although it might be helpful in monitoring severity of disease). However, in cardiac applications, $\mathrm{T}_{2}$ has been shown to increase in response to inflammation, due to increased water content related to edema, and has the tendency to decrease in response to fibrosis due to a reduced water content. ${ }^{(15,16)}$ Unfortunately, $\mathrm{T}_{2}$ is rarely included in renal MRI studies.

A second reason to measure $T_{2}$ is, that $T_{2}$ is crucial in the interpretation of BOLD (blood oxygen level-dependent) MRI data. BOLD MRI is one of the most famous MRI measures thanks to its use in brain activation studies (functional MRI). In the kidneys it is widely used as a measure of tissue oxygenation, but technically it is not. Like $\mathrm{T}_{1}$ and $\mathrm{T}_{2}, \mathrm{R}_{2}^{*}$ (the actual thing that is measured in BOLD MRI) is sensitive to a lot of things, including blood (not tissue) oxygenation.

$\mathrm{R}_{2}{ }^{*}$ is closely related to $\mathrm{T}_{2}$ and the mathematical description of this is simple if you remember that $T_{2}=1 / R_{2}\left(\mathrm{R}_{2}\right.$ is called the transverse relaxation rate and MR physicists have the tendency to use $\mathrm{T}_{2}$ and $\mathrm{R}_{2}$ interchangeably to save them the effort of writing “1/"):

$$
\begin{gathered}
R_{2}^{*}=R_{2}+R_{2}^{\prime} \\
\text { Or: } \\
R_{2}^{*}=\frac{1}{T_{2}}+R_{2}^{\prime}
\end{gathered}
$$

As outlined above, $\mathrm{T}_{2}$ is a very sensitive though not very specific measure of pathology. $\mathrm{T}_{2}{ }^{*}$ directly depends on $\mathrm{T}_{2}$. So when you want to use BOLD or $\mathrm{R}_{2}{ }^{*}$ in clinical reality as opposed to a research setting, and you cannot be absolutely sure that no other pathologies are in play, you have to measure $\mathrm{T}_{2}$ in addition!

$\mathrm{R}_{2}^{\prime}$ is the relevant part of $\mathrm{R}_{2}^{*}$ : the part that actually is sensitive to blood oxygenation. This sensitivity arises from the difference in magnetic properties of oxygenated hemoglobin (oxyHb) and deoxygenated $\mathrm{Hb}$ (deoxyHb). deoxyHb disturbs the magnetic field surrounding veins which leads to an increase in $\mathrm{R}_{2}{ }^{*}$, but it does not influence $\mathrm{T}_{2}$. So an increase in $\mathrm{R}_{2}^{*}$ is generally interpreted as hypoxia. OxyHb itself does not influence $R_{2}^{*}$ that much. This leads to some counterintuitive behavior of $\mathrm{R}_{2}^{*}$ if you consider it "a measure of tissue oxygenation". If the fractional blood volume within the tissue declines, also the amount of deoxyHb will decrease, which might lead to a decrease in $\mathrm{R}_{2}{ }^{*}$ (or $\mathrm{R}_{2}{ }^{\prime}$ ). This you might erroneously interpret as an increase in oxygenation. ${ }^{(17)}$ 


\section{MRI Techniques}

Diffusion Weighted Imaging (DWI)

Diffusion is the random motion of (water) molecules inside a medium, in our case the kidneys. In the pelvis for example, the molecules can move freely in all directions. But the motion might be restricted in for example the medulla, where most motion will be in the direction of the loop of Henle or collecting ducts. The motion is said to be anisotropic (as opposed to isotropic in the pelvis). This directionality of motion of water molecules can be measured using MRI with a technique called diffusion tensor imaging (DTI), which is a form of diffusion weighted imaging (DWI). The degree of anisotropy is denoted by the "fractional anisotropy" or FA, which ranges from 0-1. As expected, this number is low inside the pelvis and high in the medulla. The renal cortex does not favor one particular direction for diffusion, thanks to the tortuosity of the vessels and tubules there. Therefore, FA is lower in the cortex than in the medulla.

A diffusion constant is defined as a measure of how far a water molecule on average moves. In MRI, this might be called the apparent diffusion constant (ADC), the mean diffusivity (MD) or just the diffusion coefficient (D). Those parameters differ slightly from each other. In short, the MD is a more precise approximation of the ADC, because more diffusion directions are used in its calculation. It arises together with the FA from a DTI analysis. D arises from an "intravoxel incoherent motion" (IVIM) analysis. This method aims to eliminate the contribution of flow in small vessels from the diffusion. Therefore D does not include flow in small vessels, while the other two measures do. So if a lot of vessels are present, D will be lower than ADC and MD. The contribution of small vessels is also quantified separately in IVIM and denoted as perfusion fraction (PF). Annoyingly, those naming conventions are not set in stone. When the methods section of a paper says they performed DWI, they will report ADC and sometimes FA. If it mentions IVIM either in the acquisition or in the post-processing, they will report D and FP. For DTI, MD and FA will be reported, and sometimes beautiful tensor images will be shown (Figure 2). 


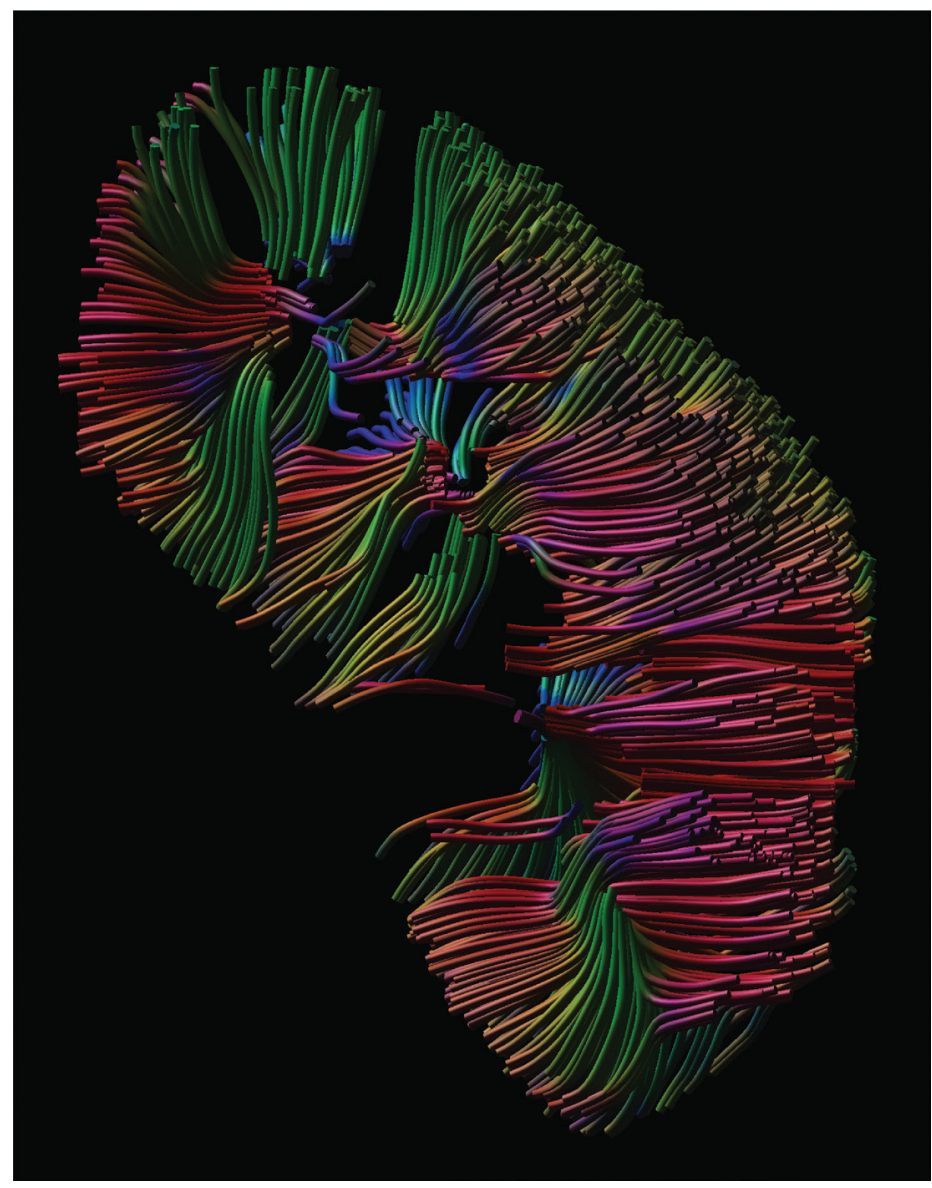

To measure this directionality of diffusion, in MRI gradients are used. With "gradient" we mean a magnetic field which depends on the location inside the body: it is, for example, stronger on the right side than on the left side. The tiny magnets we discussed earlier, will then rotate (precess) faster on the right side compared to the left side. As long as they don't move, they will precess just as fast as their immediate neighbors (we are thinking on a molecular scale right now). That also means that on the short term (milliseconds) they all precess in synchrony. Imagine now that due to diffusion one of those tiny magnets starts to move to the right. Thanks to the gradient, the magnetic field is increasing and the tiny magnet starts to precess quicker. However, the new neighbors of the tiny magnet we are studying were precessing at this speed from the beginning, so they are pointing already at 6 o'clock while our own tiny magnet is only at 4 o'clock (Figure 3). So the tiny magnets are not synchronized anymore due to diffusion and that we can measure as a decrease in signal. The more of those tiny magnets are moving along the direction of the gradient (from left to right and vice-versa) the larger is the decrease in signal. We can repeat this in a lot of different directions to measure diffusion in each direction. If there are 
large differences in the directions, like in the medulla, the fractional anisotropy (FA) is large. And if the signal decay is very large, a lot of diffusion is happening and D, $\mathrm{ADC}$ and $\mathrm{MD}$ are large. We can also repeat it with a lot of different gradient strengths (for a strong gradient, the difference in precession speed will be large between left and right). In that way, we can tune for large displacements (small gradients) or small displacements (large gradients). The strength of the gradients is given by the b-value and should be reported in the methods section. Generally, more b-values result in a better approximation of the diffusion coefficient.

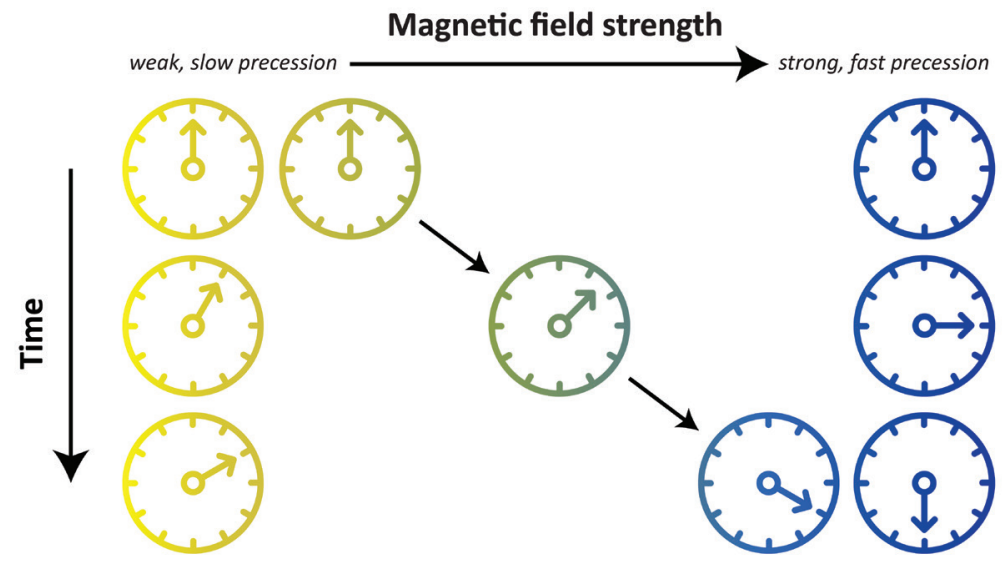

So, what can those constants be used for? Numerous diseases restrict diffusion. During inflammation, there is an influx of leukocytes leading to a reduction of the free path of water molecules - so the diffusion decreases. The same is true for fibrosis, due to an increase in extracellular matrix. Also the microstructure of the kidney will become disrupted, leading to a reduced difference in FA between cortex and medulla. The perfusion fraction FP might also be of interest to quantify microvascular perfusion, but it is difficult to measure and high coefficients of variation are reported in repeatability studies (see chapter 3 ).

\section{Phase Contrast (PC) MRI to Measure Flow Velocity}

Phase contrast MRI and DWI are not that different. They both use gradients to measure motion. In two dimensional phase contrast (2D PC), those gradients are only applied in one direction. Recall the tiny magnet which moved from left to right in the previous section? When it arrived there, it's neighbors were pointing to 6 o'clock while our magnet only was at 4 o'clock. The position on the clock is called the phase in MR terminology, so the neighbors gained more phase then our own magnet (they are at 6 and she is only at 4). Now it turns out that the difference in phase gain of our magnet and its neighbors is proportional to its velocity, if you choose the gradients in a smart way. This means you can calculate the velocity by simply multiplying the 
phase with some number, and this number, called the "encoding velocity" or venc, you can choose on the MR system.

Using this approach we can map the velocity in all voxels (MRI pixels) inside a vessel, in our case usually the renal artery. Thanks to the pulsatility of flow inside the renal artery, it is crucial to do this at different points in the cardiac phase (usually 15-25). ${ }^{(18)}$ One then obtains a graph with a peak for the high-velocity systolic flow (see chapter 3). By multiplying the mean flow over time in all voxels within the renal artery with the area of the cross section of the artery, one obtains the renal blood flow (RBF).

Phase contrast (PC) MRI can be used to measure renal blood flow (RBF) by measuring the velocity of the blood through the renal artery. Sometimes instead of the renal artery the renal vein is measured. Strictly the flow through the renal vein does not give the RBF because some urine is produced as well, but this of course only is a small fraction of total RBF. Alternatively, the flow through the aorta might be measured just above and just below the renal arteries. Subtracting the latter from the first also yields RBF. If RBF is known, renal vascular resistance (RVR) can be calculated if the systemic blood pressure is measured. The RVR is not used much in clinical practice, because its measurement usually involves a lengthy paraaminohippurate clearance procedure to quantify RBF. It however is promising as an early marker of renal disease, as illustrated by some very old papers showing that healthy subjects at risk for development of hypertension due to genetic predisposition already had increased RVR. ${ }^{(19)}$ With MRI, RBF can be quantified in a few minutes.

\section{Arterial Spin Labeling (ASL) to Measure Renal Cortical Perfusion}

Arterial spin labeling can be used to measure perfusion in the renal cortex locally, without the use of any exogenous contrast agent. The latter is relevant since another technique to measure perfusion locally, dynamic contrast enhanced imaging or DCE MRI, does use exogenous gadolinium based contrast agent (GBCA). The noninvasiveness is mainly advantageous for patients with advanced kidney disease, in whom the use of exogenous contrast agents is considered unsafe. Unfortunately, measurement of medullary perfusion is not reliable with ASL. ${ }^{(20)}$

In arterial spin labeling the spins (which is the official name for the tiny magnets) inside the feeding artery are magnetically labelled, pretty much as the name implies. "Magnetically labelling spins" just means that they are flipped around by 180 degrees, so if the tiny magnets initially pointed to the head of the patient, they now point to the feet. After some delay, called the "inflow time" or the "post labeling delay", a quick image of the kidneys is made. The delay is needed to give the labeled blood some time to travel into the kidneys. Next, this whole process is repeated, only now without the labeling - which yields the control image. The label and control image are slightly different, and this difference is caused exclusively by the different magnetization 
of the inflowing blood. Hence, the image which is obtained by subtracting the label image from the control image is called the perfusion weighted image.

In reality, the difference between the label and control image is so small that we need to repeat this process for around 15-20 times and average the results to obtain a perfusion weighted image which does not look like random noise. This causes some issues, because living patients breath, and breathing causes motion in between the images, which can be as much as a few centimeters. Subtraction of images which are misaligned by a few centimeters generally does not give good-looking results, so this has to be corrected for. The realignment of the images is called image registration. Finally, to actually quantify the perfusion, some complicated modelling is needed which incorporates amongst other measures the $\mathrm{T}_{1}$ of the kidney. ${ }^{(21)}$ Therefore, also $\mathrm{T}_{1}$ has to be measured for accurate perfusion measurement with ASL.

Unfortunately, the time needed for blood to reach the renal medulla is a few seconds, and the magnetization of labeled blood has returned to normal by then. Therefore, measurement of medullary perfusion can currently not be performed in a reliable way using ASL. ${ }^{(20)}$

\section{Dynamic Contrast Enhanced MRI}

In DCE MRI, a quick series of images of the kidney is made during the infusion of a gadolinium based contrast agent. The images are taken every few seconds during around 5-7 minutes. Using those images, for every location in the kidneys a curve is generated showing an initial increase in renal signal, followed by a gradual decrease (the time intensity curve, TIC). The exact shape of this curve differs per tissue type (Figure 4): in the cortex, arterial flow is pretty high so the signal goes up fast when the contrast agent enters.

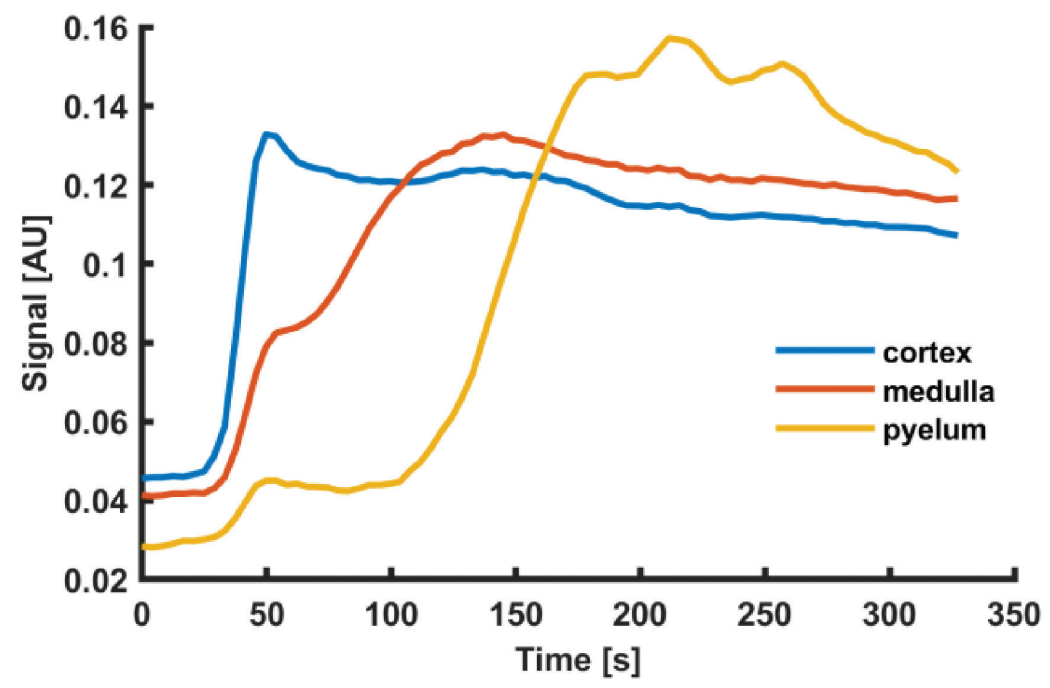


In the medulla, the signal gradually increases, followed by a gradual decrease when the contrast agent is slowly excreted. These curves can be used in a mathematical model to obtain measurements of perfusion, but also of glomerular filtration. ${ }^{(22)}$ This enables measurement of single kidney GFR. With the current models, usually time intensity curves obtained from either the entire renal parenchyma or the entire cortex are used. Therefore, per kidney one number is obtained for perfusion and one for filtration, and perfusion or filtration maps are not made. Since the most used models are not valid in the renal medulla, this technique is unable to measure perfusion in the medulla.

\section{Dixon Based Fat Quantification}

A Dixon ${ }^{3}$ scan can be used to quantify the amount of fat in tissue. The technique is mostly used in liver and muscle and is not yet validated in kidneys. ${ }^{(23)}$ Clinically, fat content in the renal parenchyma and pelvis both correlates with and contributes to renal disease. ${ }^{(24,25)}$

The tiny magnets in water and fat molecules precess on slightly different frequencies when a magnetic field is applied. In the Dixon technique, this difference is employed to achieve near-complete fat suppression on MR images (radiologists typically are not interested in fat but it tends to have a very high signal on MR images). The difference also can be used to measure the fat percentage within a voxel. (26)

\section{Field Strength: $1.5,3$ or 7 T?}

In every MRI paper it is mentioned, somewhere in the "Materials and Methods" section: what MRI system was used? Was it a 1.5, 3 or $7 \mathrm{~T}$ scanner? The "T" stands for Tesla, which is the unit in which strength of a magnetic field is measured. For comparison, the Earth's magnetic field ranges from 25-65 $\mu \mathrm{T}$ and a fridge magnet is around $5 \mathrm{mT}$. Ten years ago, $3 \mathrm{~T}$ typically was considered to be "high field", since most clinical scanners were $1.5 \mathrm{~T}$ or less. $7 \mathrm{~T}$ might be characterized as "ultrahigh field". Currently, human MRI scanners exist up to field strengths of $11.7 \mathrm{~T}$, so the term "(ultra)high field" becomes increasingly relative.

For clinical MR systems, there is usually a " 5 gauss line" drawn on the floor around the scanner. "Gauss" is just another unit to measure magnetic fields, ${ }^{4}$ and one gauss equals $0.1 \mathrm{mT}$. The space outside the 5 gauss line is considered safe for

$3 \quad$ Named after the inventor.

4 The unit "gauss" is actually named after the same person who invented the normal, or Gaussian, distribution, the German mathematician and physicist Johann Carl Friedrich Gauss. 
the general public (for example, people with pacemakers). The MR room should be designed such that it fully contains the 5 gauss line.

A fridge magnet is made from ferromagnetic material, which retains its magnetization when it is magnetized (placed in a magnetic field). Unlike fridge magnets, the magnet of a typical MRI scanner is not permanent. Most clinical MRI systems use electromagnets to create their main magnetic field. An electromagnet is nothing more than a coil of some conducting material, copper for example, carrying an electric current. Every electric current generates a magnetic field in its surroundings, as you might remember the 'right-hand rule' from high-school physics. The strength of the magnetic field scales with the strength of the electric current and the distance to the electric current. To generate magnetic fields in the range of the field strengths of clinical MRI systems, huge currents are needed. When copper wires would be used for those magnets, the electrical resistance of the wire would result in huge energy losses, resulting in heating of the wires. Therefore, superconducting materials are used. Superconductivity was invented in 1911 by a Dutchman, Heike Kamerling Onnes, who received a Nobel Prize for this work. ${ }^{(27)}$ He found that when he cooled a mercury wire to $4.2 \mathrm{~K}\left(-269^{\circ} \mathrm{C}\right)$ using liquid helium the electrical resistance suddenly dropped to zero. Using superconducting materials, the huge currents needed for clinical MRI systems become achievable. The only disadvantage is that those MRI systems have to be cooled with liquid helium, which is costly in terms of the helium itself and the energy needed for the cooling system.

So, what is the difference? The reason why it is tempting to build (ultra)high field scanners, is that theoretically, the MR signal increases with the square of the field strength. This is partly because the speed at which the spins (tiny magnets in the human body) precess, scales linearly with the magnetic field strength, and partly because of the increased magnetization at higher fields. ${ }^{(28)}$ (Remember the tiny magnets which could either point up or down? At higher fields, more will point in the same direction which increases the magnetization.) This increase in signal can be used to enhance spatial resolution or to decrease scan time. However, there are also disadvantages associated with "high" fields. For example, the magnetic field is typically less homogeneous. This especially is problematic in the abdomen, where air in the gastrointestinal tract induces local variations in the magnetic field - which is more of a problem at $3 \mathrm{~T}$ compared to $1.5 \mathrm{~T}$. When moving to even higher fields, $7 \mathrm{~T}$ for example, more factors come into play (see chapter 8 ). Regarding clinical use, some implants might be contraindicated at $3 \mathrm{~T}$, while they can be scanned at $1.5 \mathrm{~T}$. Side effects of MRI scans also increase with field strength. In general, side effects of MRI are temporary, and resolve quickly after the examination. Common side effects include vertigo, a metallic taste, nausea and muscle twitching. Apart from muscle twitching, those side-effects are mostly induced by the movement of the patient 
in and out of the bore, because during this movement a changing magnetic field is experienced. Since the change in magnetic field is larger at high fields, those sideeffects are more pronounced at $7 \mathrm{~T} .{ }^{(29)}$ Muscle twitching can be induced during the examination due to the rapid switching of the gradients, but it does not appear to be more severe nor frequent at higher field strengths. ${ }^{(29)}$ 


\section{References}

1. de Boer A, Hoogduin JM, Blankestijn PJ, Li X, Luijten PR, Metzger GJ, et al. 7 T renal MRI: challenges and promises. Magma. 2016;29(3):417-33.

2. Brachemi S, Bollee G. Renal biopsy practice: What is the gold standard? World J Nephrol. 2014;3(4):287-94.

3. Ljungberg B., Bensalah K., A. B. Guidelines on renal cell carcinoma. 2015 [cited $202031 \mathrm{Jan}$ ]:[58 p.]. Available from: http://uroweb.org/wp-content/uploads/EAUGuidelines-Renal-Cell-Cancer-2015-v2.pdf.

4. Tendera M, Aboyans V, Bartelink ML, Baumgartner I, Clement D, Collet JP, et al. ESC Guidelines on the diagnosis and treatment of peripheral artery diseases: Document covering atherosclerotic disease of extracranial carotid and vertebral, mesenteric, renal, upper and lower extremity arteries: the Task Force on the Diagnosis and Treatment of Peripheral Artery Diseases of the European Society of Cardiology (ESC). Eur Heart J. 2011;32(22):2851-906.

5. Moore K.L., Dalley A.F., A.M.R. A. Clinically oriented antomy. 6 ed. Philadelphia: Wolters Kluwer Lippincot Williams and Wilkins; 2010.

6. De Jong P.E., Koomans H.A., J.J. W. Klinische Nefrologie. Amsterdam: Reed Business; 2011.

7. El-Reshaid W, Abdul-Fattah H. Sonographic assessment of renal size in healthy adults. Med Princ Pract. 2014;23(5):432-6.

8. Rhoades R.A., D.R. B. Medical Physiology: priniciples for clinical medicine. 4 ed. Baltimore: Lippincott Williams and Wilkins; 2013.

9. el-Galley RE, Keane TE. Embryology, anatomy, and surgical applications of the kidney and ureter. Surg Clin North Am. 2000;80(1):381-401, xiv.

10. Brezis M, Rosen S. Hypoxia of the renal medulla--its implications for disease. N Engl J Med. 1995;332(10):647-55.

11. Prasad PV, Edelman RR, Epstein FH. Noninvasive evaluation of intrarenal oxygenation with BOLD MRI. Circulation. 1996;94(12):3271-5.

12. Mahon B. The man who changed everything. Hoboken, NJ, USA: John Wiley \& Sons Ltd; 2004.

13. Wolf M, de Boer A, Sharma K, Boor P, Leiner T, Sunder-Plassmann G, et al. Magnetic resonance imaging $\mathrm{T} 1$ - and T2-mapping to assess renal structure and function: a systematic review and statement paper. Nephrol Dial Transplant. 2018;33(suppl_2):ii41-ii5o.

14. Franke M, Baessler B, Vechtel J, Dafinger C, Hohne M, Borgal L, et al. Magnetic resonance T2 mapping and diffusion-weighted imaging for early detection of cystogenesis and response to therapy in a mouse model of polycystic kidney disease. Kidney Int. 2017;92(6):1544-54. 
15. Matsumoto S, Mori H, Miyake H, Yamada Y, Ueda S, Oga M, et al. MRI signal characteristics of progressive massive fibrosis in silicosis. Clin Radiol. 1998;53(7):510-4.

16. Kim PK, Hong YJ, Im DJ, Suh YJ, Park CH, Kim JY, et al. Myocardial T1 and T2 Mapping: Techniques and Clinical Applications. Korean J Radiol. 2017;18(1):113-31.

17. Niendorf T, Pohlmann A, Arakelyan K, Flemming B, Cantow K, Hentschel J, et al. How bold is blood oxygenation level-dependent (BOLD) magnetic resonance imaging of the kidney? Opportunities, challenges and future directions. Acta Physiol (Oxf). 2015;213(1):19-38.

18. Villa G, Ringgaard S, Hermann I, Noble R, Brambilla P, Khatir DS, et al. Phasecontrast magnetic resonance imaging to assess renal perfusion: a systematic review and statement paper. MAGMA2019.

19. Textor SC, Turner ST. Renal vascular response to sodium loading in sons of hypertensive parents. Hypertension. 1991;17(6 Pt 2):982-8.

20. Nery F, Buchanan CE, Harteveld AA, Odudu A, Bane O, Cox EF, et al. Consensus-based technical recommendations for clinical translation of renal ASL MRI. MAGMA2019.

21. Buxton RB, Frank LR, Wong EC, Siewert B, Warach S, Edelman RR. A general kinetic model for quantitative perfusion imaging with arterial spin labeling. Magn Reson Med. 1998;40(3):383-96.

22. Annet L, Hermoye L, Peeters F, Jamar F, Dehoux JP, Van Beers BE. Glomerular filtration rate: assessment with dynamic contrast-enhanced MRI and a corticalcompartment model in the rabbit kidney. J Magn Reson Imaging. 2004;20(5):843-9.

23. Kukuk GM, Hittatiya K, Sprinkart AM, Eggers H, Gieseke J, Block W, et al. Comparison between modified Dixon MRI techniques, MR spectroscopic relaxometry, and different histologic quantification methods in the assessment of hepatic steatosis. Eur Radiol. 2015;25(10):2869-79.

24. Spit KA, Muskiet MHA, Tonneijck L, Smits MM, Kramer MHH, Joles JA, et al. Renal sinus fat and renal hemodynamics: a cross-sectional analysis. MAGMA2019.

25. de Vries AP, Ruggenenti P, Ruan XZ, Praga M, Cruzado JM, Bajema IM, et al. Fatty kidney: emerging role of ectopic lipid in obesity-related renal disease. Lancet Diabetes Endocrinol. 2014;2(5):417-26.

26. Hussain HK, Chenevert TL, Londy FJ, Gulani V, Swanson SD, McKenna BJ, et al. Hepatic fat fraction: MR imaging for quantitative measurement and display--early experience. Radiology. 2005;237(3):1048-55.

27. Heike Kamerlingh Onnes - Facts: Nobel Media AB; 2020 [updated 2020; cited 2020 31 Jan]. Available from: https://www.nobelprize.org/prizes/physics/1913/onnes/ facts/.

28. Redpath TW. Signal-to-noise ratio in MRI. The British journal of radiology. 1998;71(847):704-7.

29. Heilmaier C, Theysohn JM, Maderwald S, Kraff O, Ladd ME, Ladd SC. A large-scale study on subjective perception of discomfort during 7 and 1.5 T MRI examinations. Bioelectromagnetics. 2011;32(8):610-9. 


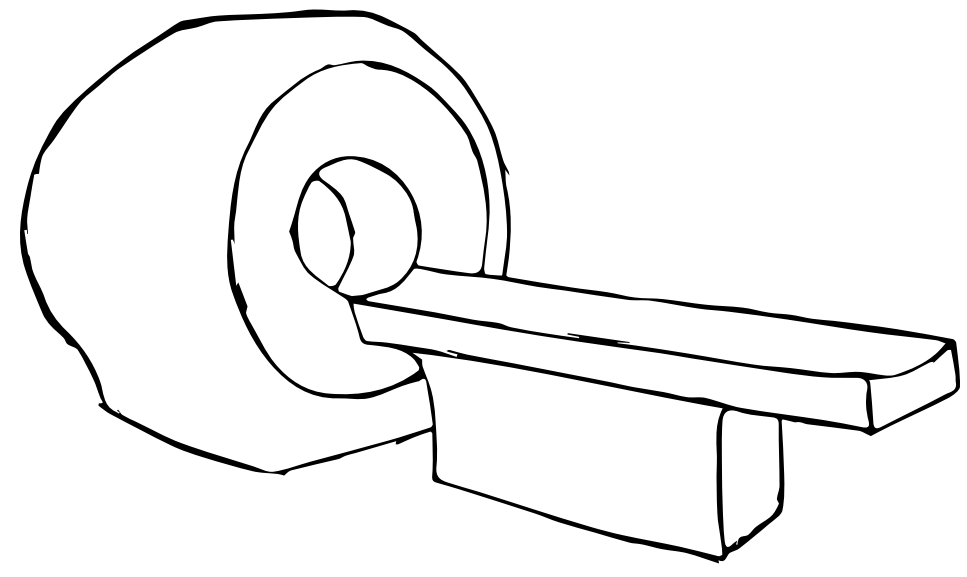




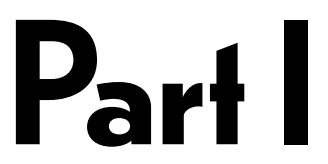

Technical Development 



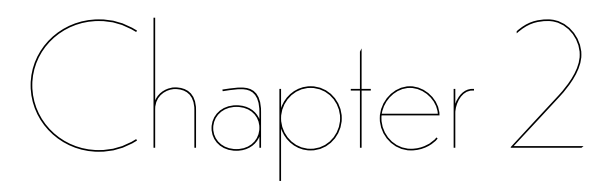

\section{Multi-Parametric Renal MRI - an Intra-Subject Test-Retest Repeatability Study}

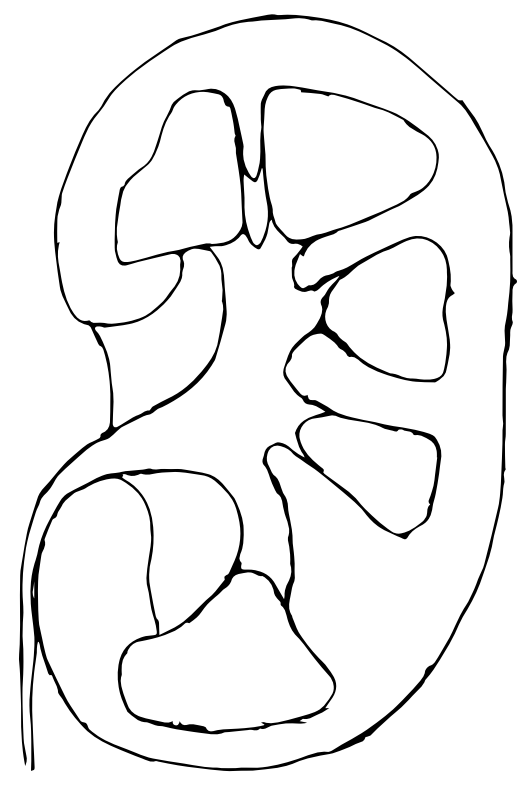

\section{Author contributions}

de Boer A, HarteveldAA, Stemkens B, Blankestijn PJ, Bos C, Franklin SL, Froeling M, Joles JA, Verhaar MC, van den Berg N, Hoogduin H, Leiner T

$$
\text { Published in }
$$

J Magn Reson Imaging 2020; doi:10.1002/jmri.27167 


\section{Abstract}

Background Renal multi-parametric magnetic resonance imaging (MRI) is a promising tool for diagnosis, prognosis and treatment monitoring in kidney disease.

Purpose To determine intra-subject test-retest repeatability of renal MR measurements

Study type Prospective

Population 19 healthy subjects aged over 40 years

Fieldstrength and sequences $T_{1}$ and $T_{2}$ mapping, $R_{2}^{*}$ mapping or blood oxygenation level-dependent (BOLD) MRI, diffusion tensor imaging (DTI) and intravoxel incoherent motion (IVIM) diffusion weighted imaging (DWI), 2D phase contrast, arterial spin labelling (ASL), dynamic contrast enhanced (DCE) MRI and quantitative Dixon for fat quantification at $3 \mathrm{~T}$.

Assesment Subjects were scanned twice with approximately one week between visits. Total scan time was $\sim 1$ hour. Post-processing included motion correction, semi-automated segmentation of cortex and medulla and fitting of the appropriate signal model.

Statistical tests To assess repeatability, a Bland-Altman analysis was performed and coefficients of variation (CoVs), repeatability coefficients and intra-class correlation coefficients were calculated.

Results CoVs for relaxometry $\left(\mathrm{T}_{1}, \mathrm{~T}_{2}, \mathrm{R}_{2}^{*} / \mathrm{BOLD}\right)$ were below $6.1 \%$, with lowest CoVs for $\mathrm{T}_{2}$ maps and highest for $\mathrm{R}_{2}^{*} / \mathrm{BOLD}$. CoVs for all diffusion analyses were below $7.2 \%$, except for perfusion fraction $\left(\mathrm{F}_{\mathrm{p}}\right)$, with CoVs ranging from $18-24 \%$. The $\mathrm{CoV}$ for renal sinus fat volume and percentage were both around $9 \%$. Perfusion measurements were most repeatable with ASL (cortical perfusion only) and 2D phase contrast with CoVs of 10 and $13 \%$, respectively. DCE perfusion had a CoV of $16 \%$, while single kidney GFR had a CoV of $13 \%$. RCs ranged from 7.7-87\% (lowest/highest values for medullary mean diffusivity and cortical FP, respectively) and ICCs ranged from -0.010.98 (lowest/highest values for cortical FP and renal sinus fat volume, respectively).

Data conclusion CoVs of most MRI measures of renal function and structure (with the exception of $\mathrm{F}_{\mathrm{p}}$ and perfusion as measured by DCE) were below $13 \%$, which is comparable to standard clinical tests in nephrology. 


\section{Introduction}

Multi-parametric magnetic resonance imaging (MRI) of the kidneys is a promising tool for diagnosis, prognosis and treatment monitoring in kidney disease. Contrary to anatomic imaging, functional imaging allows for quantitative measures of oxygenation, perfusion, tissue microstructure and water content. Such variables are likely to change in the course of various conditions and are therefore sensitive measures of pathology. ${ }^{(1)}$ Rising interest in renal MRI is driven in Europe by the COST action PARENCHIMA, dedicated to magnetic resonance imaging biomarkers in kidney disease (www.renalmri.org). The ultimate goal of this collaboration of kidney MRI researchers is the initiation of large-scale clinical studies needed to confirm the value of kidney MRI as a clinical biomarker. ${ }^{(1)}$

In various diseases, functional MRI has already been successfully applied to detect pathologic changes. For example diffusion weighted imaging (DWI), an often used MRI method, shows a decrease in the apparent diffusion constant (ADC) with increasing fibrosis in chronic kidney disease (CKD). ${ }^{(2)}$ In kidney transplants, the ADC is consistently decreased in patients with acute tubular necrosis, acute rejection and immunosuppressive toxicity, but the ADC is not able to differentiate between these pathologies. ${ }^{(3)}$ In comparison, tissue $\mathrm{T}_{2}$ for example is known to increase in response to inflammation, ${ }^{(4)}$ while it tends to decrease in reaction to severe fibrosis, ${ }^{(5)}$ which enables discrimination between those conditions.

Variants of DWI include diffusion tensor imaging, focused on the directionality of diffusion, and intravoxel incoherent motion (IVIM), which corrects for contribution of microperfusion to the diffusion coefficient and additionally estimates a perfusion fraction. With ASL, cortical perfusion can be mapped, which is known to be impaired in for example chronic kidney disease (CKD). ${ }^{(6)}$ Using PC MRI, renal blood flow can be measured. BOLD MRI is sensitive oxygenation and can detect renal hypoxia, which is thought to be the driving factor behind the progression of CKD. ${ }^{(7)} \mathrm{T}_{1}$ and $\mathrm{T}_{2}$ are both sensitive to changes in microstructure, water content and oxygenation and are therefore sensitive, though not very specific markers of pathology. ${ }^{(8)}$ Introduction of other MRI sequences in patient care might increase the specificity of MRI as a diagnostic tool. Ultimately, multiparametric evaluation could allow for recognition of patterns in MR parameters characteristic for different pathologies.

As a first step towards large diagnostic and prognostic studies, the repeatability of kidney MRI has to be determined.(1) Although for most of those techniques repeatability studies have been performed, repeatability data of multiparametric MRI in a single group of subjects is scarce as is data on how repeatability compares between different sequences and methods. Furthermore, as far as we are aware, repeatability data on renal quantitative Dixon and renal $\mathrm{T}_{2}$ mapping are not yet 
available apart from a conference proceeding for measurement of $\mathrm{T}_{2}{ }^{(9)}$

In this multiparametric study, we additionally included Dixon based fat quantification and dynamic contrast enhanced (DCE) imaging. Fat content in both the renal parenchyma and the renal sinus is thought to contribute to progression of kidney disease. ${ }^{(10)}$ DCE MRI can be used to quantify perfusion and to measure glomerular filtration rate (GFR), the main clinical measure of kidney function. ${ }^{(11)}$

The aim of this study was to determine the test-retest repeatability of multiparametric kidney MR in healthy subjects. A secondary aim was to compare the repeatability of different MR perfusion techniques, i.e. phase contrast MRI, arterial spin labeling (ASL) and dynamic contrast enhanced (DCE) MRI).

\section{Methods}

\section{Subjects}

This study was approved by the local institutional review board and all subjects signed informed consent prior to inclusion. For subjects to be included, they had to be aged 40 years or older. Exclusion cirteria were a history of renal or cardiovascular disease and contra-indications for MRI, including incompatible implants, claustrophobia or an allergy to gadolinium based contrast agents. Subjects were imaged twice with approximately one week between visits. Data of insufficient image quality on visual assessment were excluded. Both visits of each subject were planned around the same time of the day. Subjects were asked to avoid salt and protein rich meals and to drink 2L per 24 hours of non-alcoholic liquids on the day of the scan to roughly standardize hydration and dietary conditions. Prior to one imaging session, usually the first, blood was sampled to measure creatinine, cystatin $\mathrm{C}$ and hematocrit, to allow calculation of estimated glomerular filtration rate (eGFR) using the CKD-EPI formula ${ }^{(12)}$ (based on creatinine and cystatin C).

\section{Imaging Protocol}

All subjects were scanned on a 3 T MR system (Ingenia, Philips Healthcare, Best, The Netherlands; software release 5.3.1) with a 12-channel posterior and a 16-channel anterior receive coil array. The imaging protocol started with a localizer scan. $\mathrm{B}_{\mathrm{o}}$ and $\mathrm{B}_{1}$ shimming was performed for all acquisitions. All scans were acquired in the coronal plane with a Cartesian readout, except for the DCE, which was acquired in the transverse plane with a radial readout. A detailed overview of the imaging parameters is listed in Table 1. 


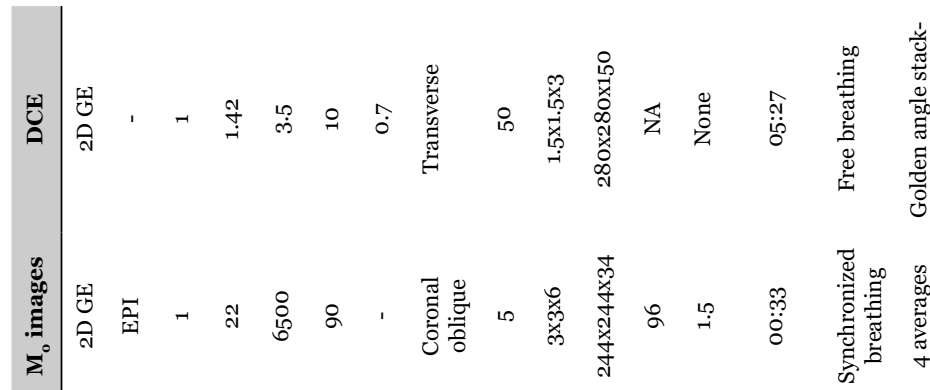

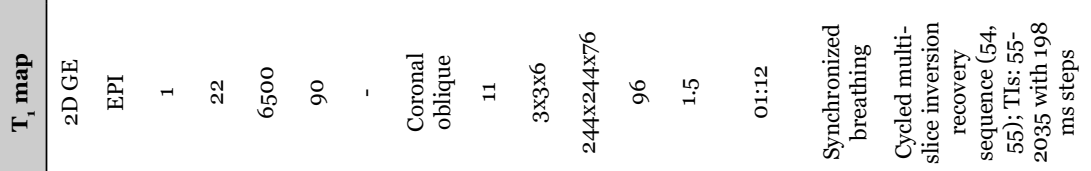

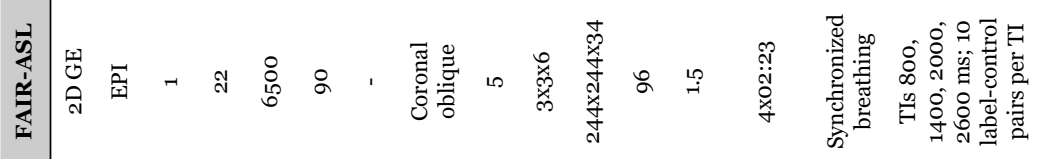

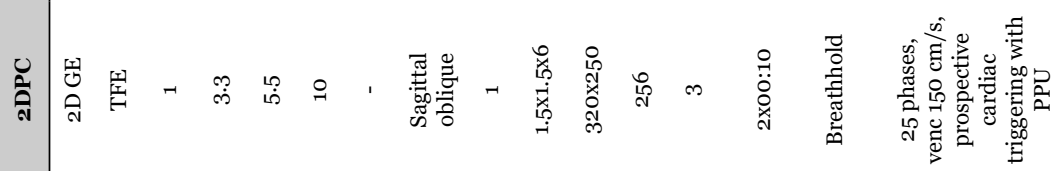

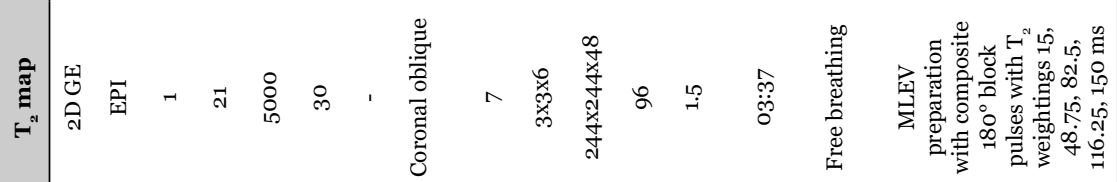

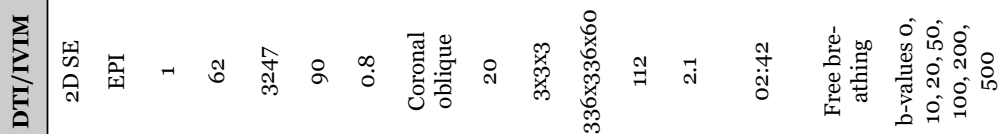

*

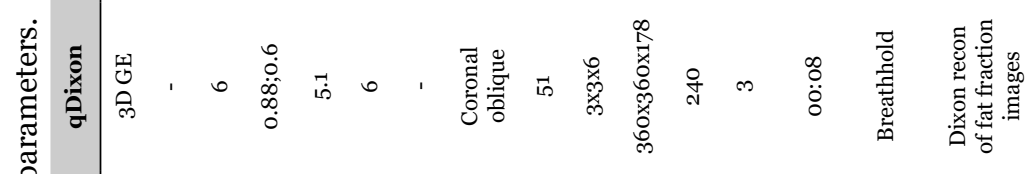

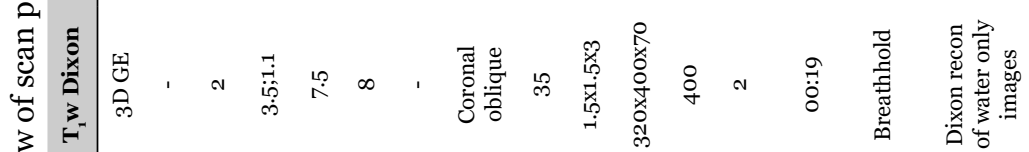

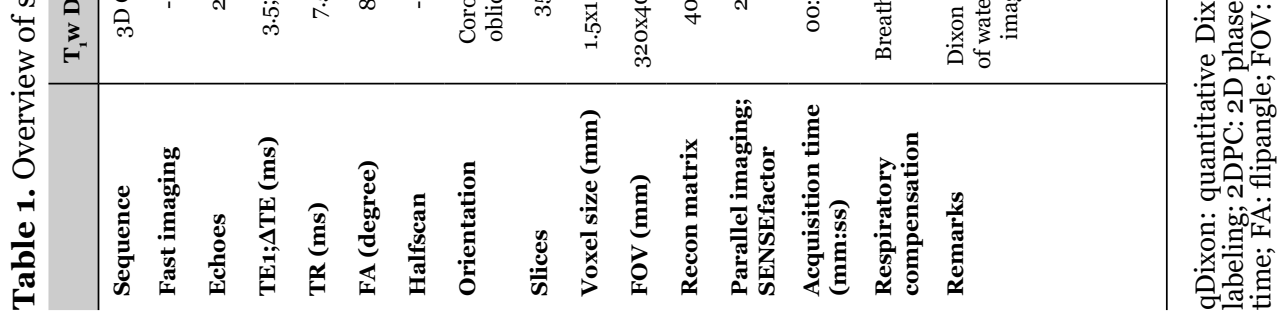


Data was acquired either during breath-hold (PC, BOLD MRI, the anatomical and quantitative Dixon), synchronized breathing (the subject was asked to breath in between the single-shot acquisitions, ASL, Mo and $\left.\mathrm{T}_{1}\right)$ or free breathing $\left(\mathrm{T}_{2}\right.$ mapping, DWI, DCE MRI). Total scan time was approximately 1 hour. During the last sequence (DCE MRI), $0.05 \mathrm{mmol} / \mathrm{kg}$ of gadobutrol (Bayer Healthcare, Berlin, Germany) was infused at a rate of $1 \mathrm{~mL} / \mathrm{s}$, followed by a saline flush of $20 \mathrm{~mL}$. DCE MRI had a temporal resolution of $4.1 \mathrm{~s}$ and images were acquired continuously during 5:27 minutes. Care was taken to acquire $\mathrm{T}_{1}$ maps, ASL images and $\mathrm{M}_{\mathrm{o}}$ images using the same field of view and voxel size, since these images had to be combined for ASL quantification. For timing of the QUIPPS settings in the FAIR-ASL acquisition, please refer to ${ }^{(13)}$. For the first four patients a slightly different scan protocol for $\mathrm{T}_{1}$ mapping and FAIR-ASL was used. Those $\mathrm{T}_{1}$ maps and ASL scans were therefore excluded from the current analysis.

\section{Post-processing}

Post-processing was performed using in-house developed software in Matlab (R2015b, Mathworks Nattick, MA, USA), unless stated otherwise. All images were converted to NIfTI format. ${ }^{(14)}$ On the $\mathrm{T}_{1}$ weighted anatomical Dixon images coordinates of the center of both kidneys were identified manually. These were used in all series to make a wide crop around each kidney. Remaining processing was performed separately for each kidney.

\section{Image Reconstruction}

All images were reconstructed online using the scanner software except for the radial stack-of-stars DCE data, which was reconstructed offline in Matlab using the GRASP compressed sensing algorithm. ${ }^{(15)}$ In short, this algorithm exploits temporal sparsity using a first-order temporal total variation constraint. DCE data were reconstructed using 21 radial spokes per volume, resulting in a spatial resolution of $1.5 \times 1.5 \times 3.0 m^{3}$ and a temporal resolution of 4.1s. This was the highest temporal resolution SNR would allow as judged by one expert reader (B.S.).

\section{Motion Correction}

Respiratory motion compensation (registration) was performed for all scans except for BOLD, anatomical and quantitative Dixon scans, since those were acquired in breath-hold. Image registration was achieved by groupwise deformable registration in Elastix (version 4.9.0 (16,17)). This was performed slice-wise for all coronal 2D scans and image-wise for the DCE, which was acquired in $3 \mathrm{D}$. A principal component analysis (PCA) based ${ }^{(14)}$ similarity measure was used, since it is insensitive for the sometimes strong contrast changes in the images, especially in the $\mathrm{T}_{1}$ and DCE series. For the phase contrast images, registration was performed on the magnitude images 
to provide sufficient detail to the registration algorithm.

For ASL quantification, the $\mathrm{T}_{1}$ series and Mo images were required. Since these scans were all acquired with the same geometry settings, these series were registered all at once using the same groupwise approach as described above. For the repeatability analysis of $\mathrm{T}_{1}$, the $\mathrm{T}_{1}$ series was also processed separately.

\section{Segmentation}

To allow separate analysis of cortex and medulla segmentation, masks for both regions were defined for all kidneys. Masks were generated using k-means clustering on the DCE, $\mathrm{T}_{1}$ and BOLD series and copied to the other series as described in the supplementary materials. ${ }^{(23)}$ If necessary, they were manually adapted by one expert (B with 5 years of experience in renal imaging) in ITK-SNAP ${ }^{(24)}$. Areas affected by artefacts, as identified by visual inspection, were avoided. Detailed information on mask generation, including the approach to copy the masks to the other series, is provided in the supplementary materials. For examples of the cortex and medulla mask for different readouts, see supplementary Figure S1. Segmentation of the renal artery in the phase contrast data was performed semi-automated (see supplementary materials). On the quantitative Dixon scans, the renal sinus was manually delineated to enable quantification of renal sinus fat.

\section{Quality Assessment}

Quality assessment was performed by three readers (A.B., M.F. and H.H. with 5,7 , and 8 years of experience in renal imaging) according to the criteria in the supplementary materials. Data judged to be of insufficient quality by reader A.B. were presented to readers M.F. and H.H. If they agreed, the data were discarded. Examples of discarded images are shown in the supplementary materials, Figure S1.

\section{Modelling}

Relaxometry data ( $\mathrm{T}_{1}, \mathrm{~T}_{2}$ and $\mathrm{R}_{2}{ }^{*}$ mapping) was fitted to mono-exponential models in a voxel-wise manner, yielding a relaxation time or rate constant map and a So map (see supplementary materials).

For the analysis of DWI data two models were used, see (20); a DTI and a biexponential IVIM model. The DTI analysis yields the mean diffusivity (MD) and fractional anisotropy (FA). The IVIM model measures also a diffusion constant (D), excluding the contribution of microperfusion (measured as FP).

For modelling of ASL data the Buxton model was used, as previously described (13). This model yields perfusion per unit of tissue and the arterial transit time (ATT), or the time blood needs to travel from the labelling location (usually the aorta) to tissue.

Modelling of the DCE data was performed on a whole-parenchyma basis (as 
opposed to voxel-by-voxel fitting). A renal two-compartment model was used ${ }^{(1,21,22)}$. The model was fitted in two steps: ${ }^{(1)}$ tubular flow (GFR per unit of tissue volume) and the tubular transit time were fitted to the second part of the data followed by ${ }^{(2)}$ fitting of the vascular parameters (blood volume, bolus arrival delay and plasma transit time) to the first part of the data with fixed tubular parameters. A similar approach was proposed by Tofts et al. ${ }^{(11)}$ For details, please refer to the supplementary materials. For DCE MRI, parenchymal perfusion, mean residence time (MRT, plasma transit time plus bolus arrival delay ${ }^{(11)}$ ) and single kidney GFR (skGFR) were reported. Perfusion was calculated as blood volume divided by mean residence time.

Fat quantification was performed on the scanner using a six-echo Dixon approach with vendor provided software ${ }^{(23)}$. This yielded a fat fraction (FF) which was multiplied with sinus volume to obtain renal sinus fat volume.

\section{Statistical Analysis}

For the MR measurements, mean values obtained from the first and second scan were separately compared for cortex and medulla on a per-kidney base. To assess repeatability, within subject coefficients of variations $\left(\mathrm{CoV}_{\mathrm{w}}\right)$ were calculated as follows ${ }^{(24)}$ :

$$
\operatorname{CoV}_{w}=100 \% * \sqrt{\frac{1}{2 N} \sum_{n=1}^{N} \frac{\left(x_{1, n}-x_{2, n}\right)^{2}}{\bar{x}_{n}^{2}}}
$$

Here, $\mathrm{x}_{1}$ and $\mathrm{x}_{2}$ denote the mean value for the first and second scan. Since the analysis was performed on a per-kidney base, the summation is over kidneys, not patients. $\mathrm{N}$ and $\mathrm{n}$ denote total number of kidneys and kidney number, respectively (or arteries in case of 2D phase contrast). Additionally, intraclass correlation coefficients (ICC, two-way mixed effects, absolute agreement, single measurement) and repeatability coefficients (RC) were calculated. The RC gives the expected range of the repeated measurement in $95 \%$ of subjects and equals the range between the limits of agreement in the Bland-Altman plot. ${ }^{(25)}$ A Bland-Altman analysis was performed, including scatter plots and Bland-Altman plots with limits of agreement and corresponding confidence intervals. ${ }^{(25)}$ All analyses were performed in R version 3.4.4 ${ }^{(26)}$. Data is reported as mean (standard deviation) or mean (interquartile range), where appropriate.

\section{Results}

\section{Volunteer Characteristics}

Twenty healthy subjects were included, of whom one was scanned only once and therefore excluded from the current analysis. Subject characteristics are presented in 
Table 2. The age distribution was skewed to the left: ten subjects were aged between 40 and 50 . Of the 19 subjects, 14 were scanned with a 7 -day interval, the remaining subjects were scanned at 4, 5, 8, 14 and 16 days. eGFR was above $60 \mathrm{ml} / 1.73 \mathrm{~m}^{2} / \mathrm{min}$ in all subjects.

Table 2. Baseline characteristics of study population.

\begin{tabular}{|c|c|}
\hline & Median (IQR) \\
\hline Number of participants (male) & $19(9)$ \\
\hline Age,years & $49(45-57)$ \\
\hline eGFR, $\mathrm{mL} / 1.73 \mathrm{~m}^{2} / \mathrm{min}$ & $97(88-101)$ \\
\hline Creatinine, $\mu \mathrm{mol} / \mathrm{L}$ & $73(69-85)$ \\
\hline Cystatin C, mg/L & $0.80(0.77-0.90)$ \\
\hline Hematocrit, fraction & $0.41(0.38-0.44)$ \\
\hline
\end{tabular}

IQR: interquartile range.

\section{MRI Measurements}

An overview of all calculated repeatability measures is provided in Table 3.

\section{Relaxometry}

All acquired $\mathrm{T}_{1}$ maps were included except for one examination which had to be excluded because of severe motion obscuring the kidneys (see supplementary materials, Figure S2). Furthermore, the $\mathrm{T}_{1}$ maps of the first four subjects were excluded since they were obtained with a slightly different imaging protocol.

Some $\mathrm{R}_{2}{ }^{*}$ source images were affected by macroscopic susceptibility artifacts where air bubbles were present in stomach or intestines adjacent to the kidneys. Two $\mathrm{R}_{2}{ }^{*}$ examinations had to be excluded because of severe (respiratory) motion artifacts (see supplementary materials, Figure S2).

The $\mathrm{T}_{2}$ images sometimes suffered from banding artifacts due to sensitivity of the $\mathrm{T}_{2}$ preparation to $\mathrm{B}_{\mathrm{o}}$ inhomogeneities and insufficient fat suppression (for examples, see supplementary materials Figure S2 and $\mathrm{S}_{3}$ ), which in one kidney led to exclusion of the examination. Representative acquired and processed relaxometry images from a single subject are shown in Figure 1.

Bland-Altman plots and scatter plots for all relaxometry sequences are presented in Figure 2. Repeatability was best for $\mathrm{T}_{2}$ mapping, with a $\mathrm{CoV}$ of $2.9 \%$. For $\mathrm{R}_{2}{ }^{*}$ mapping, the $\mathrm{CoV}$ was around $6 \%$ for cortex and medulla. For $\mathrm{T}_{1}$ mapping, despite low CoVs (5.1 and 2.8\% for cortex and medulla, respectively), a small but systematic bias was present which was more pronounced in the cortex compared to the medulla. This is evident in Figure 2a-c. 


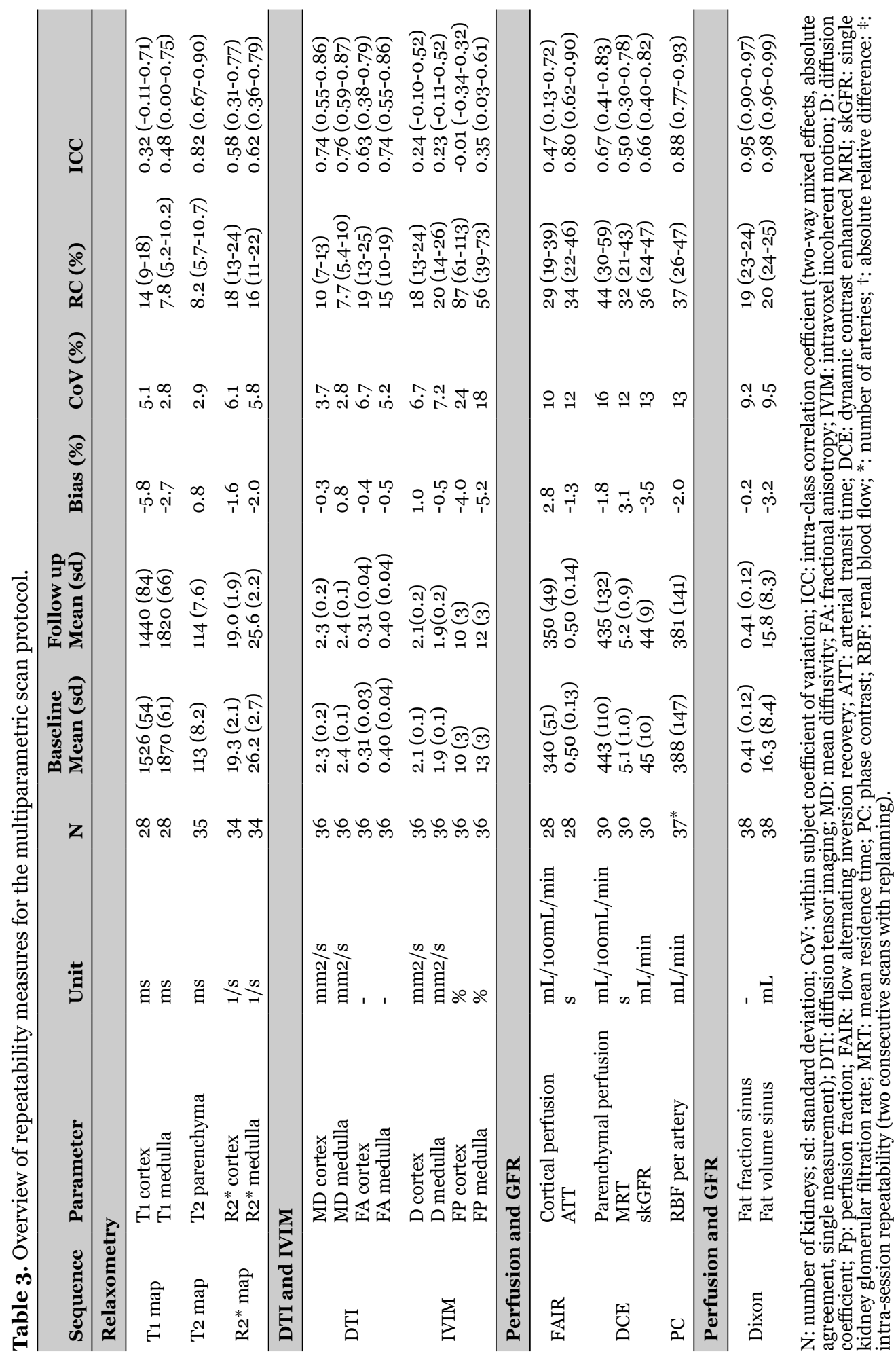




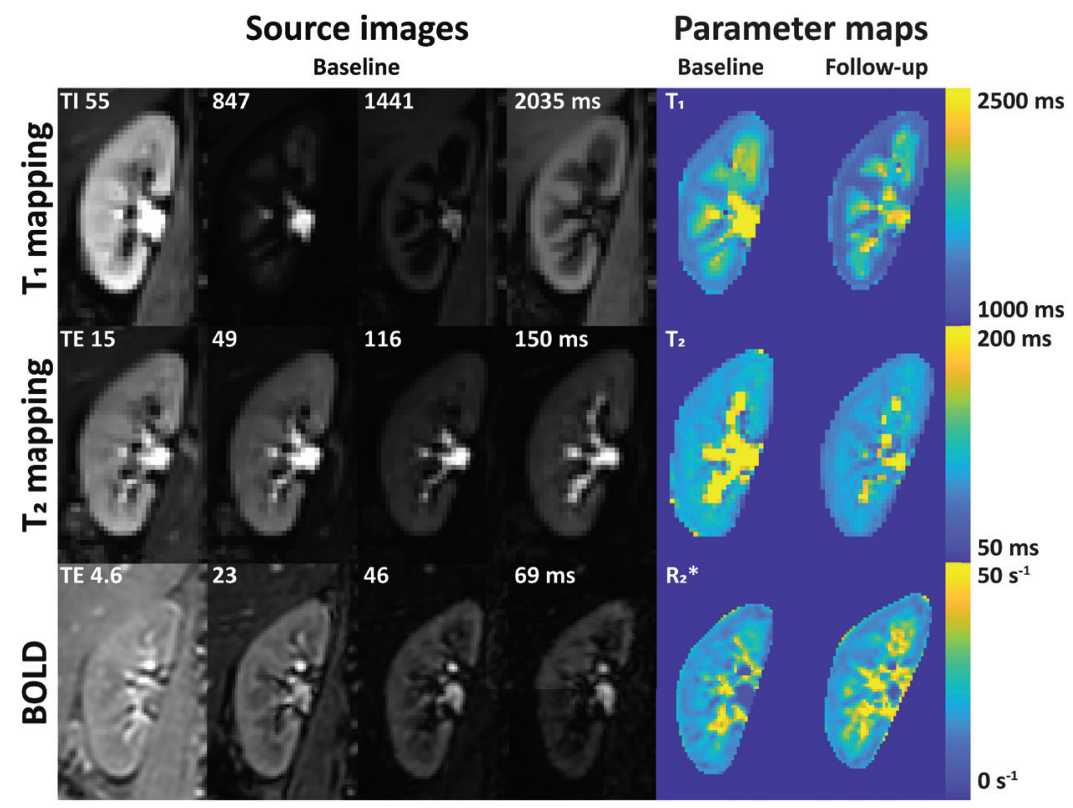

Figure 1. Source images and parameter maps of the relaxometry measures. BOLD: blood oxygen level-dependent.
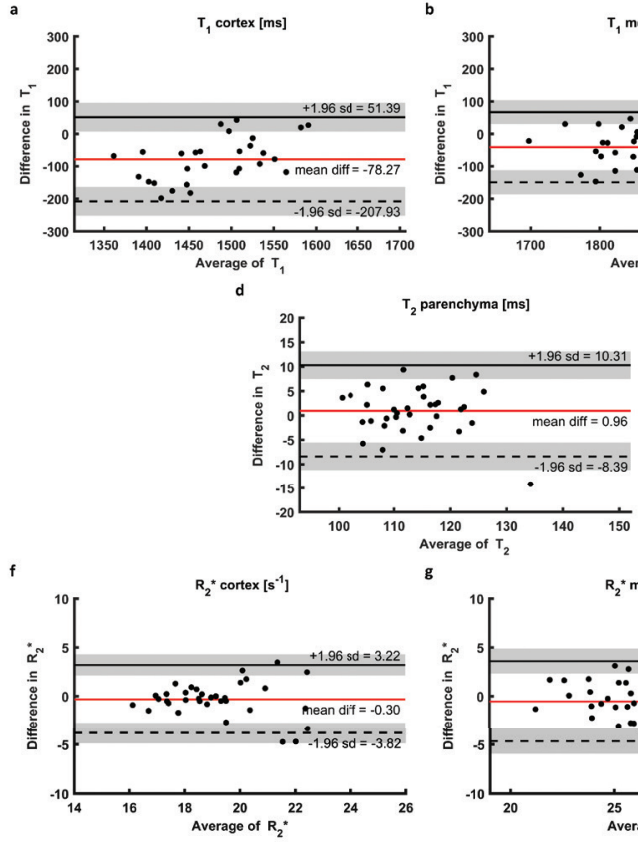
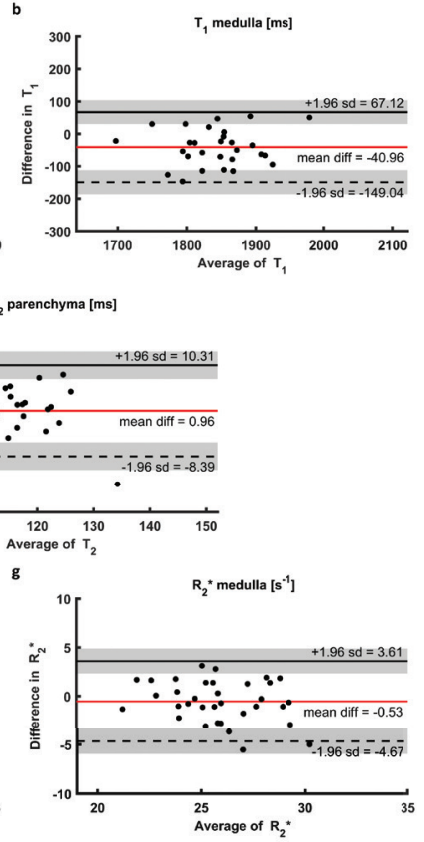
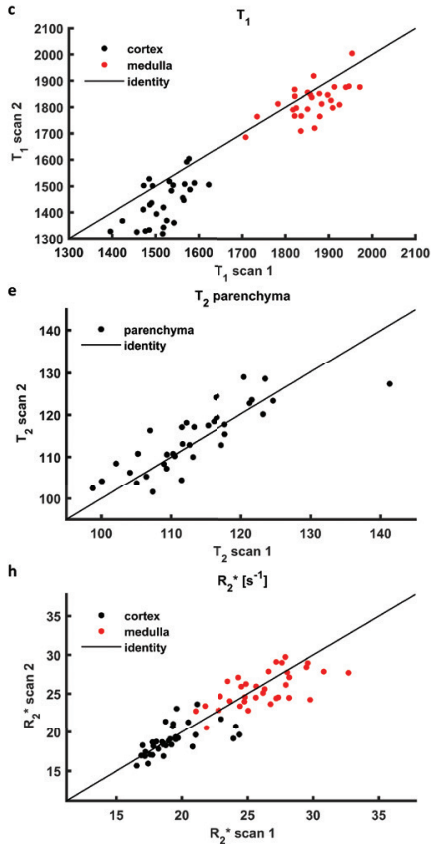

Figure 2. Bland-Altman plots and scatterplots for all relaxometry measures; a mean cortical $\mathrm{T}_{1}$; b mean medullary $\mathrm{T}_{1}$; c scatter plot for both cortical and medullary $\mathrm{T}_{1} ; \mathbf{d}$ parenchymal $\mathrm{T}_{2}$; e scatter plot of parenchymal $\mathrm{T}_{2} ; \mathbf{f}$ mean cortical $\mathrm{R}_{2}{ }^{*} ; \mathbf{g}$ mean medullary $\mathrm{R}_{2}{ }^{*} ; \mathbf{h}$ scatter plot for both cortical and medullary $\mathrm{R}_{2}{ }^{*}$. For larger image, see digital version. 


\section{Diffusion}

Diffusion images were available in all but one subject in whom DWI was not performed due to time constraints. In Figure 3, source images and parameters maps of the diffusion images are shown for the same subject as in Figure 1. Sometimes on the diffusion images the top of the kidneys were obscured by fold-over artifacts which could not be sufficiently suppressed by saturation slabs.

Bland Altman plots and corresponding scatterplots are depicted in Figure 4. Regarding the diffusion constants, the $\mathrm{CoV}$ of mean diffusivity (MD, DTI analysis) was slightly lower than the apparent diffusion constant (IVIM analysis): 3.7 versus $6.7 \%$ in the cortex and 2.8 versus $5.2 \%$ in the medulla. FA had CoVs of 6.7 and $5.2 \%$ in cortex and medulla, respectively. Repeatability of the perfusion fraction (FP, IVIM analysis) was markedly less with CoVs of 24 and $18 \%$, in cortex and medulla respectively (table 3 ).

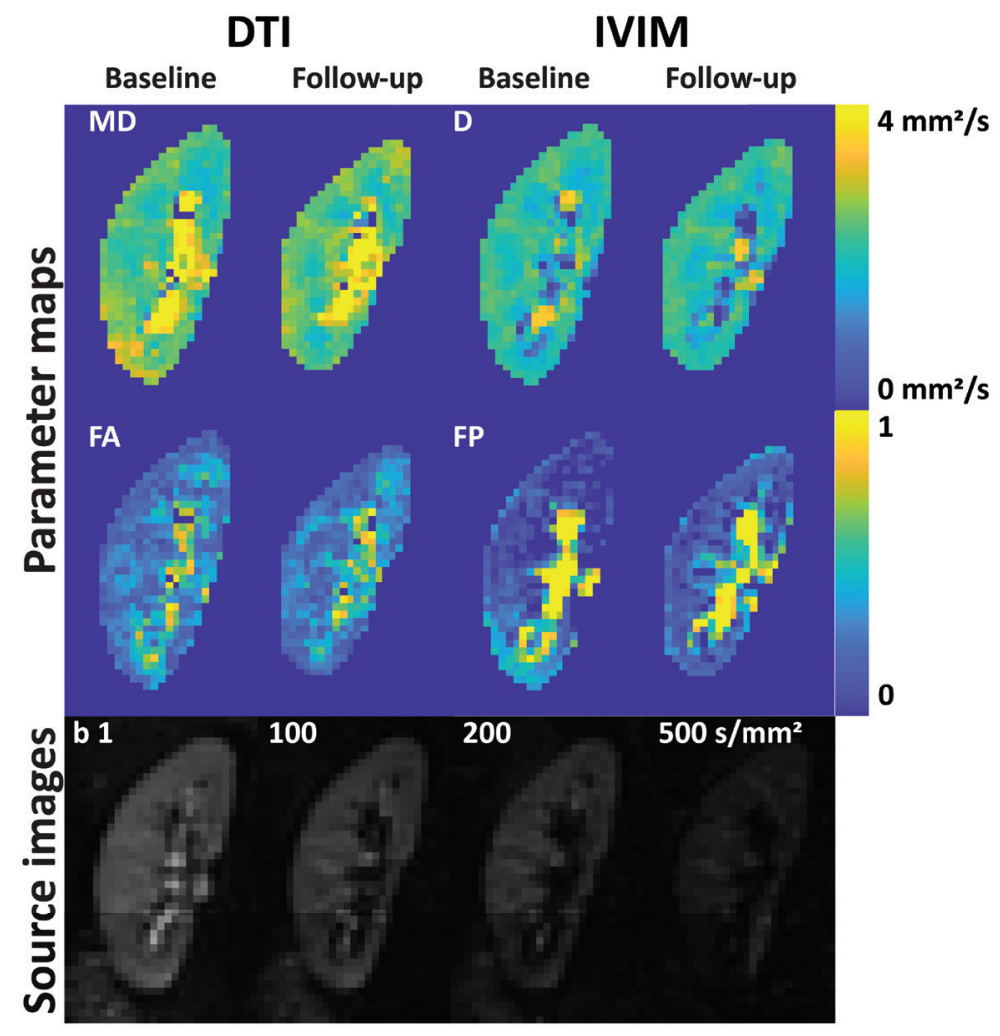

Figure 3. DTI and IVIM images. DTI: diffusion tensor imaging; IVIM: intravoxel incoherent motion; MD: mean diffusivity; D: diffusion coefficient; FA: focal anisotropy; FP: perfusion fraction. 

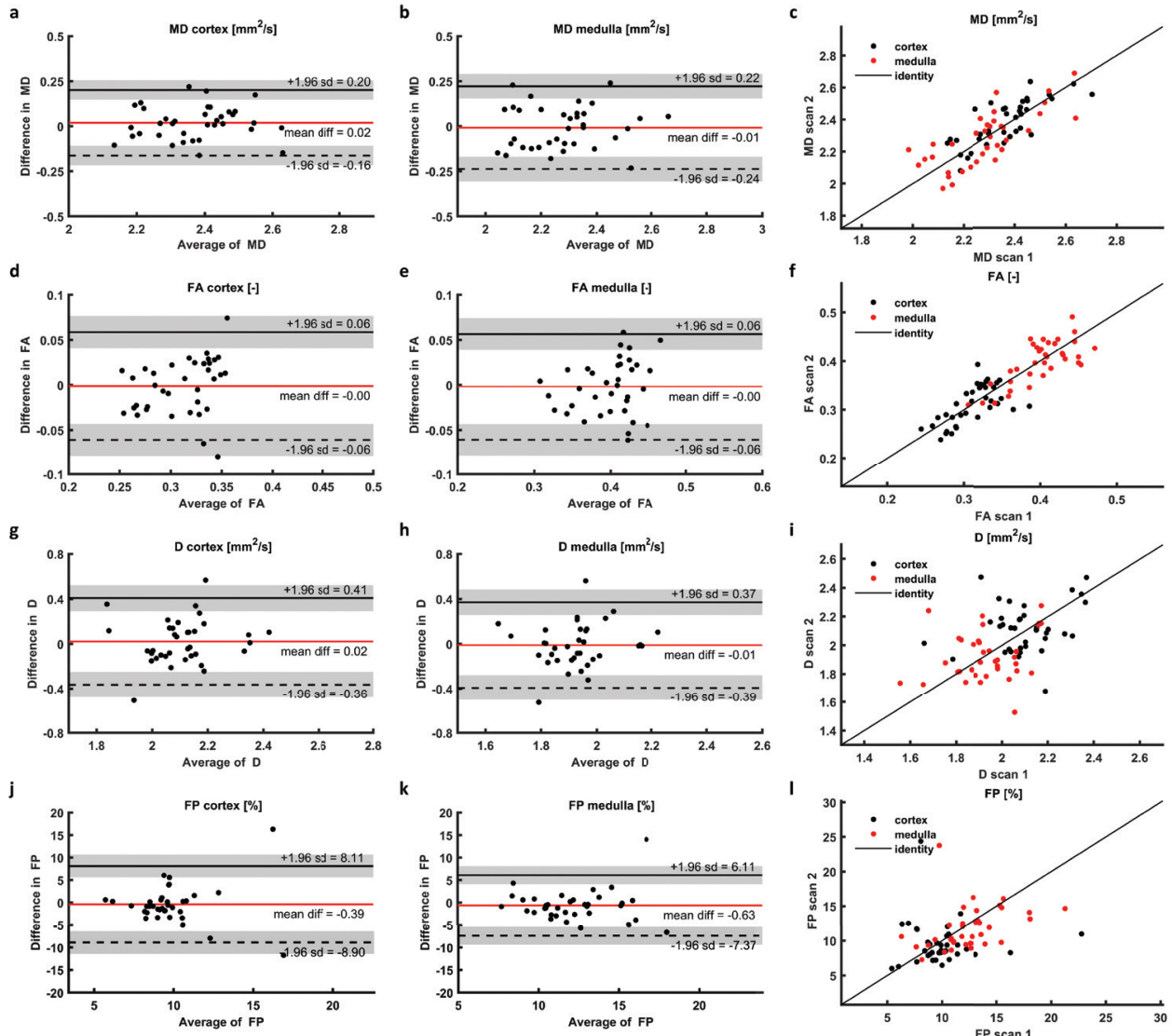

Figure 4. Bland-Altman plots and scatterplots for all DWI measures; a mean cortical MD (DTI); b mean medullary MD (DTI); c scatter plot for both cortical and medullary MD (DTI); d mean cortical FA (DTI); e mean medullary FA (DTI); f scatter plot for both cortical and medullary FA (DTI); $\mathbf{g}$ mean cortical D (IVIM); h mean medullary D (IVIM); i scatter plot for both cortical and medullary D (IVIM); a mean cortical $\mathrm{F}_{\mathrm{p}}$ (IVIM); b mean medullary $\mathrm{F}_{\mathrm{p}}$ (IVIM); c scatter plot for both cortical and medullary $\mathrm{F}_{\mathrm{p}}$ (IVIM) For larger image, see digital version. DTI: diffusion tensor imaging; IVIM: intravoxel incoherent motion; MD: mean diffusivity; D: diffusion coefficient; FA: focal anisotropy; FP: perfusion fraction.

\section{Perfusion and GFR}

For FAIR-ASL and Mo, which used the same readout , no datasets had to be excluded because of insufficient image quality. Artifacts (Figure 5) were limited to geometrical distortion due to Bo inhomogeneities and susceptibility effects due to air in the lungs and digestive tract, which did not affect the perfusion quantification. In one subject, FAIR-ASL could not be planned because the kidneys were located in the same coronal plane as the aorta. In the first four subjects FAIR-ASL was performed with slightly different settings, and therefore they were excluded from the current analysis. This resulted in 13 complete datasets for FAIR ASL. 


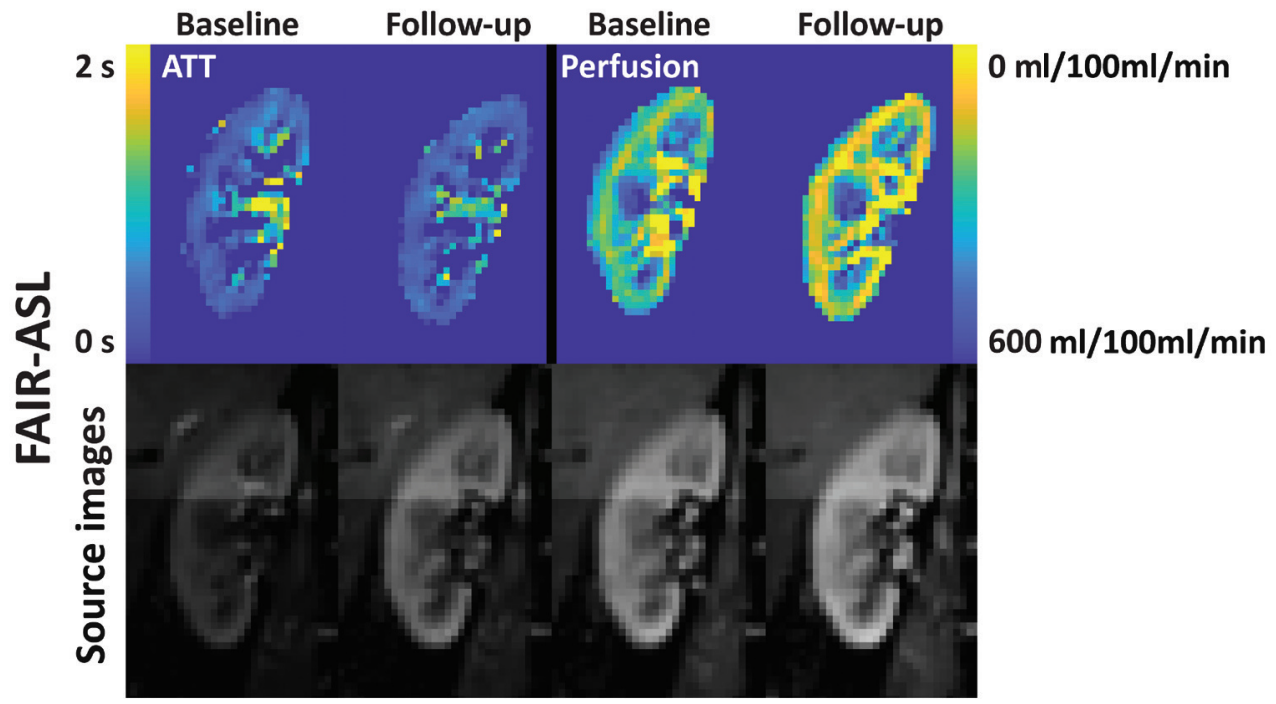

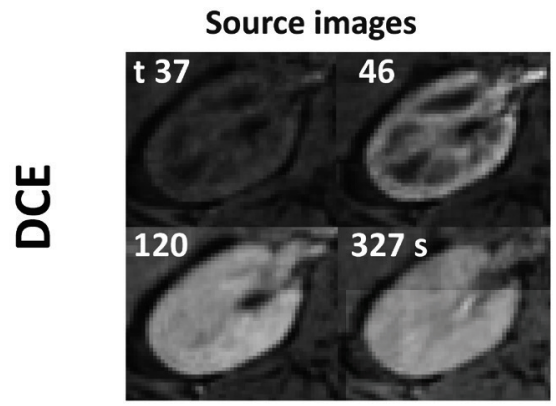

Source images

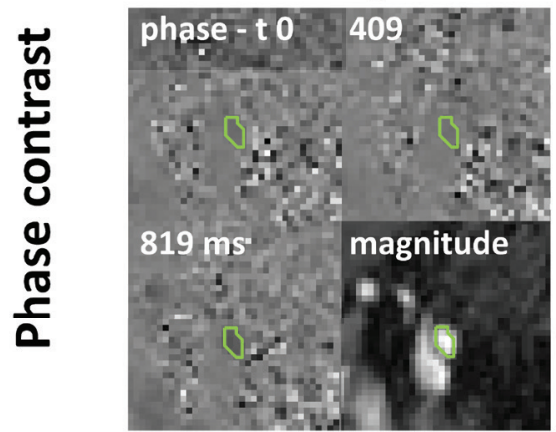

Time intensity curves

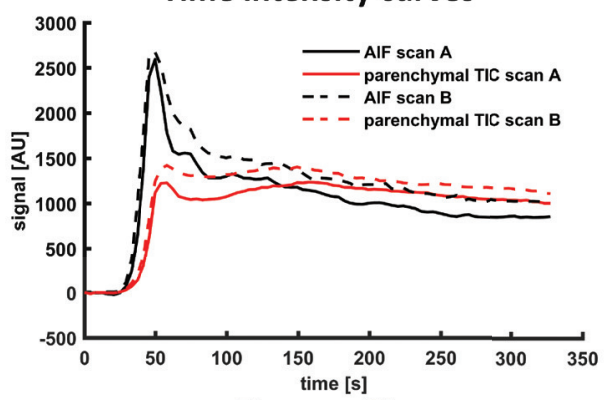

Flow profile

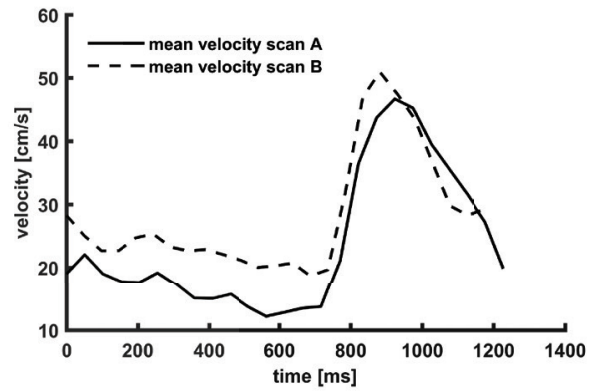

Figure 5. top Source images and parameter maps of arterial spin labeling; middle transverse source images of DCE imaging at four time points (precontrast, cortical phase, medullary phase and late phase) and the AIF and parenchymal TIC measured at both scan sessions; bottom source images (phase and magnitude) of the phase contrast scan, including the region-of-interest and blood flow velocity over the cardiac cycle as measured during the first and second scan session. FAIR-ASL: flow-attenuated alternating inversion recovery arterial spin labeling; ATT: arterial transit time; DCE: dynamic contrast enhanced MRI; AIF: arterial input function; TIC: time intensity curve; AU: arbitrary units. 
In some DCE images (Figure 5) streaking artifacts originating from the radial acquisition were visible. In two subjects DCE MRI could not be performed due to an unavailable software license, in one subject the image SNR was too low for analysis and in one subject there were problems with intravenous access, resulting in 15 complete datasets.

Although respiratory motion during the acquisition of $2 \mathrm{D}$ phase contrast sometimes resulted in misalignment of the images, it did not affect image quality (Figure 5). One subject had two renal arteries bilaterally and one subject had two left renal arteries. One subject was excluded due to failed cardiac synchronization, and two arteries were excluded due to erroneous planning (see supplementary materials, Figure S1). This resulted in 37 examined renal arteries from 18 subjects.

CoVs of all perfusion measures was generally lower compared to both relaxometry and diffusion analysis. Repeatability of perfusion obtained by ASL was better than DCE (10 vs 17\%), but the measures of transit time (ATT in ASL and MRT in DCE) were equally repeatable for both sequences (12\%). Repeatability of renal blood flow, as measured as the total blood flow through the main renal artery by $2 \mathrm{D}$ phase contrast, was comparable to that of the perfusion imaging methods with a $\mathrm{CoV}$ of $13 \%$. For Bland-Altman plots and corresponding scatter plots, see Figure 6.

\section{Fat Quantification}

Acquisition of Dixon images succeeded in all subjects (Figure 7a). Main artifacts included motion artifacts and water-fat interference artifacts. Fat quantification in the renal parenchyma did not yield realistic values, ranging from below zero to more than $\mathbf{1 0 \%}$ (for additional images, see supplementary materials Figure S3). Repeatability of both the fat fraction and the total fat volume in the renal sinus was around $9 \%$ as assessed by the CoV. For Bland-Altman plots, see Figure $7 \mathrm{~b}$-e.

\section{Robustness}

In 13 out of 19 subjects all sequences could be acquired successfully when only considering patient-related reasons for exclusion. Out of the 6 subjects where this was not the case, two had multiple scans affected by motion artifacts, in one patient FAIR-ASL could not be acquired due to anatomy, in two subjects 2DPC acquisition failed due to erroneous planning, and in one patient 2DPC failed due to erroneous cardiac triggering. Missing data due to time constraints, absence of software keys or acquisition with (slightly) different protocols were not considered patient-related and were thus excluded in the evaluation of exam robustness. 


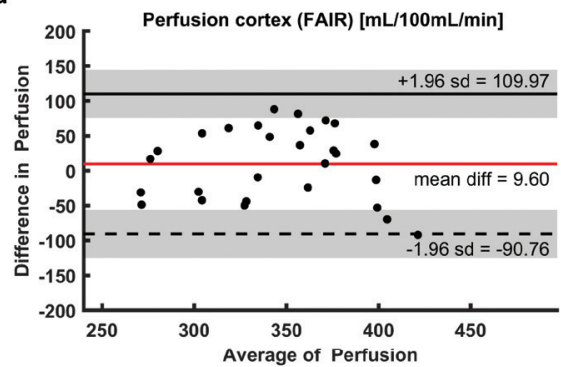

c

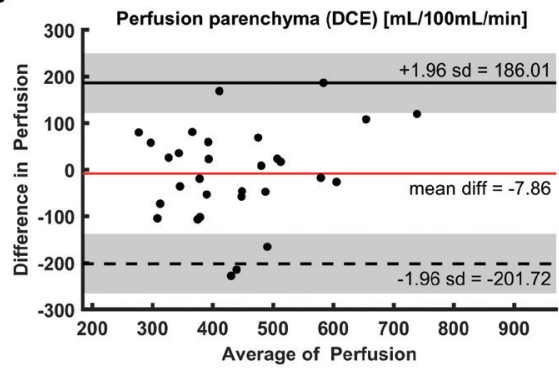

e

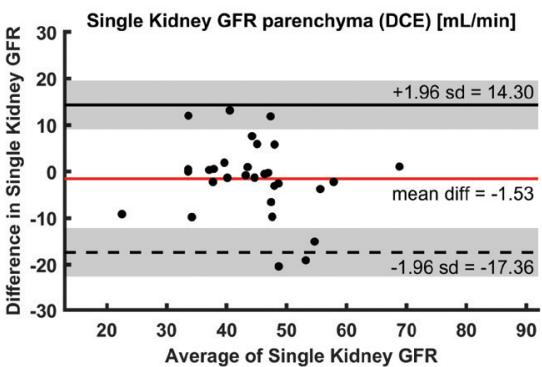

g

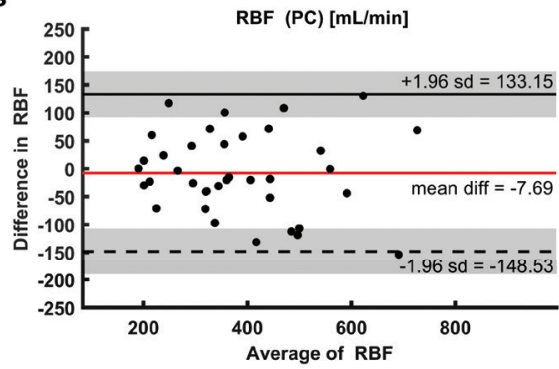

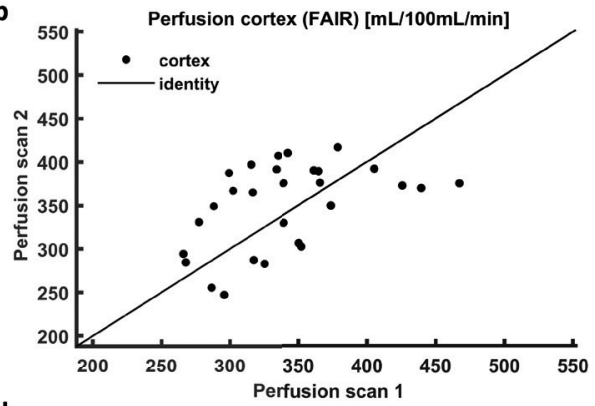

d

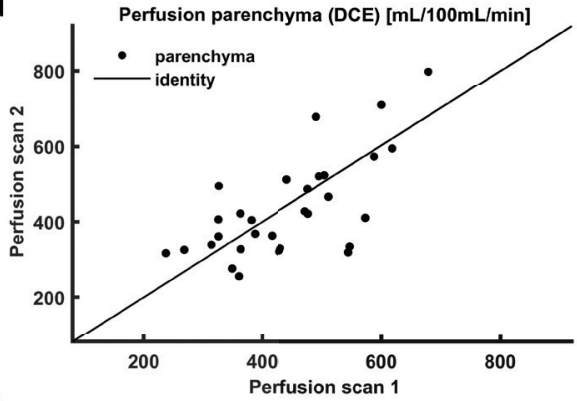

f

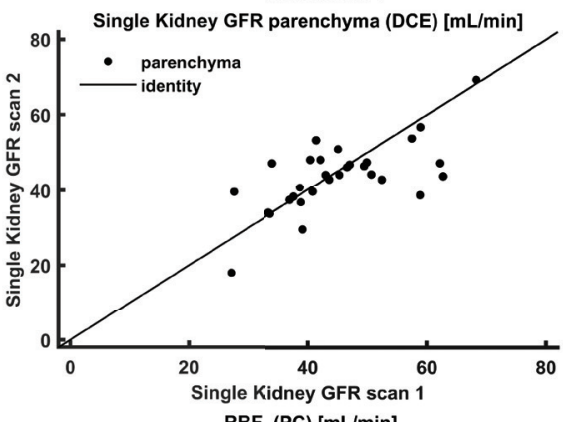

h

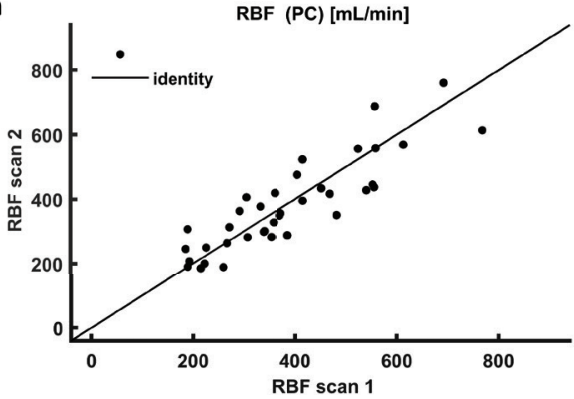

Figure 6. Bland-Altman plots and scatterplots for all perfusion measures; a Mean cortical perfusion (FAIR-ASL); b scatter plot for cortical perfusion (FAIR-ASL); c mean parenchymal perfusion (DCE); d scatter plot for parenchymal perfusion (DCE); e single kidney GFR (DCE); f scatter plot for single kidney GFR (DCE); c mean renal artery flow (phase contrast); d scatter plot for renal artery flow (phase contrast). FAIR: flow-attenuated alternating inversion recovery; ASL: arterial spin labeling; DCE: dynamic contrast enhanced MRI; GFR: glomerular filtration rate; RBF: renal blood flow; PC: phase contrast. 


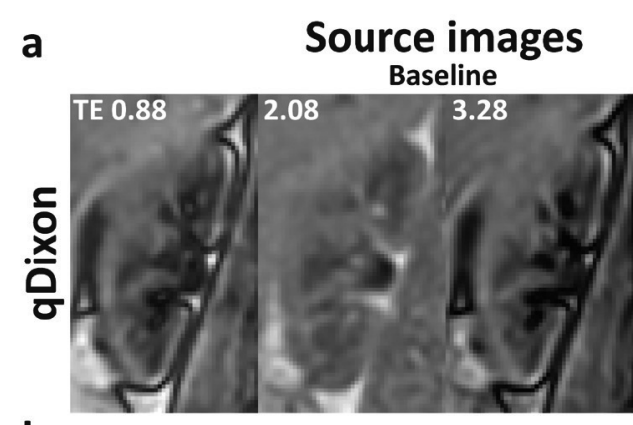

b
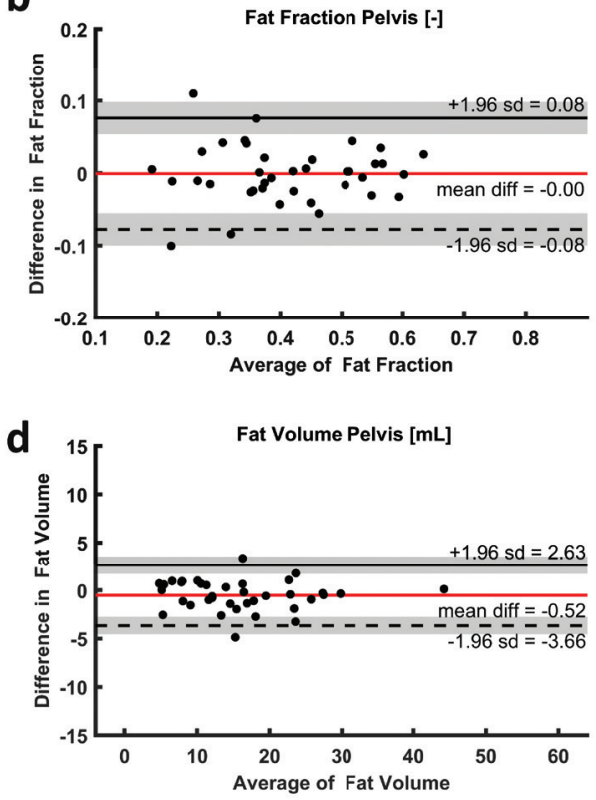

Parameter maps Baseline Follow-up

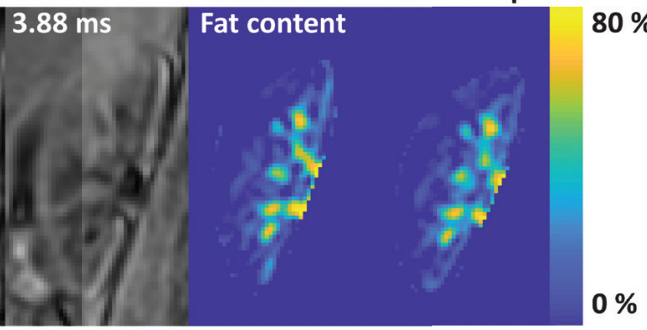

C

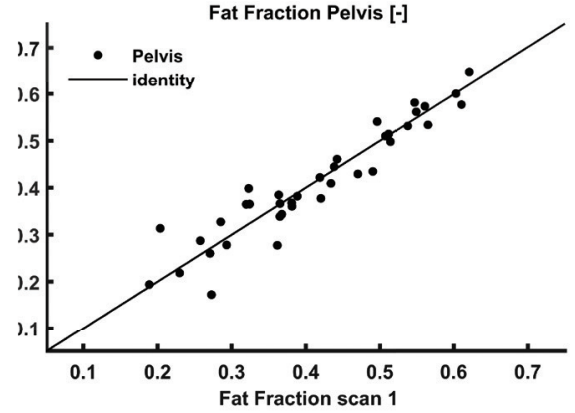

Fat Volume Pelvis [mL]

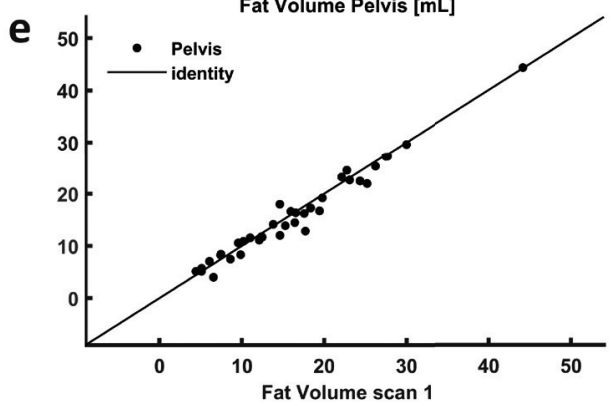

Figure 7. Source images and parameter maps of the quantitative Dixon; b-e Bland Altman plots and scatter plots for $\mathbf{b}$ pelvic fat fraction; $\mathbf{c}$ scatter plot for pelvic fat fraction; $\mathbf{d}$ pelvic fat volume; e scatter plot for pelvic fat volume. qDixon: quantitative Dixon. 


\section{Discussion}

In this study, the repeatability of various functional and structural MRI measurements of the kidney was assessed. Repeatability of most functional MRI measurements were in the range of other commonly used kidney function tests. For example, for a serum creatinine blood test a $\mathrm{CoV}$ of $5.8 \%$ has been reported (healthy subjects, monthto-month variability). ${ }^{(27)}$ Inulin clearance by constant infusion, which is considered the gold standard for GFR measurement, has been reported to have a CoV of $11.3 \%$ under strictly controlled dietary and hydration conditions. ${ }^{(28)}$

CoVs of more structural measures like $\mathrm{T}_{1}, \mathrm{~T}_{2}$ and diffusion constants were generally lower (indicating better repeatability) than those of functional measures like $\mathrm{R}_{2}^{*}$ and perfusion. $\mathrm{T}_{1}$ and $\mathrm{T}_{2}$ both had an excellent repeatability with CoVs below $5.1 \%$ for both cortex and medulla. Note that the $\mathrm{CoV}$ of $5.1 \%$ for cortical $\mathrm{T}_{1}$ was slightly higher than reported previously. ${ }^{(29-32)}$ This can likely be attributed to remaining contrast agent from the administration during the previous scan session, about a week before. ${ }^{(33)}$ This caused a significant negative bias in the follow-up measurement. For repeatability of renal $\mathrm{T}_{2}$ values, we could only find one preliminary report, which reported comparable repeatability. ${ }^{(9)} R_{2}^{*}$ mapping or BOLD MRI is sensitive to renal oxygenation and therefore a more functional measure compared to the other relaxometry measurements. This is reflected by the slightly higher $\mathrm{CoV}$, in line with previous reports. ${ }^{(29-31,34-36)}$

Considering repeatability of the diffusion data, the DTI analysis yielded lower CoVs compared to the IVIM analysis. Repeatability of FP was poor especially in the cortex, in line with previous reports. ${ }^{(29,37,38)}$ Calculation of $\mathrm{FP} \neg$ is based on a double exponential model with 4 free parameters and is therefore prone to fitting errors.

Perfusion measurements were least reproducible, which likely reflects physiological fluctuations in renal perfusion. ${ }^{(39)}$ Although ASL, DCE and PC MRI all measure slightly different perfusion indices, they can all be used to monitor changes in perfusion over time, or, in research settings, between groups. Therefore, it is relevant to compare their performance. Overall the perfusion values obtained agreed reasonably well. Cortical perfusion as measured with ASL (on average 345 $\mathrm{mL} / 100 \mathrm{~mL} / \mathrm{min}$ ) was slightly lower compared to perfusion as measured with DCE $(439 \mathrm{~mL} / 100 \mathrm{~mL} / \mathrm{min})$. Considering that renal parenchymal volume is around $15 \mathrm{OmL}$ ${ }^{(40)}$ the flow through the renal arteries $(385 \mathrm{~mL} / \mathrm{min})$ seems relatively low. However, 6 of those arteries were one of a pair of renal arteries and small accessory renal arteries might have been present which were not detected on the vascular survey. In terms of repeatability, renal blood flow as measured with 2D phase contrast MRI was relatively reliable, with a $\mathrm{CoV}$ of $13 \%$. It must be noted that $2 \mathrm{D}$ phase contrast is challenging to plan on the scanner, since the imaging slice has to be positioned exactly perpendicular to the artery. 
Cortical perfusion measurement by FAIR-ASL had lowest $\mathrm{CoV}$ of all perfusion measurements: $10 \%$, outperforming both phase contrast and DCE. Vascular transit times were equally repeatable when measured using FAIR-ASL or DCE MRI. This confirms Cutajar et al. ${ }^{(41)}$, who also reported lower CoVs for FAIR-ASL compared to DCE (16\% vs $30 \%)$. A disadvantage of FAIR-ASL is, however, that it cannot be performed on both kidneys simultaneously in a minority of subjects whose kidneys lie in the same plane as the aorta, since the aorta should not be included in the imaging plane. ${ }^{(42)}$ In accordance with a recent recommendation paper, medullary perfusion was not reported for FAIR-ASL since it was deemed unreliable due to low SNR. ${ }^{(42)}$

DCE perfusion measurements are heavily influenced by the first-pass peak of the arterial input function (AIF), which probably explains the higher CoV compared to FAIR-ASL. Accurate measurement of this peak is challenging due to the high contrast agent concentration resulting in saturation of the signal. Also, when the temporal resolution is too low, the peak can be missed. Considering this, it is surprising that with a temporal resolution of only $4.1 \mathrm{~s}$ we managed to obtain a $\mathrm{CoV}$ of only $16 \%$ for the perfusion (while reported CoVs range from $14-29.5 \%^{(11,37,41,43,44)}$ ). This is comparable to Tofts et al. ${ }^{(11)}$ and Cutajar et al. ${ }^{(44)}$, who reported CoVs of 14 and $17 \%$ respectively, both with acquisitions with a temporal resolution of 2.5 s. The relatively good repeatability found in this study might be explained by the radial acquisition, which constantly samples the center of k-space and therefore is less likely to miss the AIF peak. In Cartesian acquisitions, which are more commonly used, this is not the case. To avoid saturation of the signal, we injected the contrast agent $(1 \mathrm{~mL} / \mathrm{s})$ relatively slow probably leading to a broader and lower AIF peak. Furthermore, we gave a half dose of contrast agent, but in future studies this will be decreased to a quarter dose. Also, the two-step fitting approach, where tubular parameters were fitted to the second part of the time intensity curve and vascular parameters like perfusion to the first part, might have contributed to the better $\mathrm{CoV}$ compared to other reports.

Single kidney GFR as measured by DCE had a CoV of $13 \%$, which is slightly higher than the $\mathrm{CoV}$ of inulin clearance $\left(11.3 \%,{ }^{(28)}\right)$ and lower than other reports on DCE MRI. ${ }^{(37,43)}$ Also for tubular flow, which is multiplied by cortical volume to obtain (sk)GFR, higher CoVs are generally reported (15-18\%, ${ }^{(11,43,44)}$. Again, the twostep fitting approach might explain the relatively good repeatability. This approach was already suggested by Tofts et al. ${ }^{(11)}$, but has not yet been generally adopted. Its effectiveness should be confirmed in further analyses.

Although repeatability of the DCE measures was relatively good compared to other studies, we noted that the temporal regularization applied in the compressed sensing reconstruction affected the shape of the time intensity curves, leading to flattening of both the AIF and the parenchymal first pass peak. In principle, if the 
temporal regularization on both uptake curves can described by the same fixed temporal kernel that is weighted equally, it would cancel out. However, in the compressed sensing reconstruction the relative weight of temporal regularization can vary over the uptake curve depending on the magnitude of the data mismatch term. Thus, it likely will affect the perfusion and filtration quantification. When we compared perfusion and GFR values obtained with and without compressed sensing, we found $\sim 10 \%$ lower perfusion values and no effect on GFR with compressed sensing. Indeed, the temporal smoothing in compressed sensing mainly affects the AIF and the first pass in tissue which determine perfusion.

Measurement of renal sinus fat fraction and fat volume yielded CoVs of around $9 \%$. We could not find reports of repeatability of quantitative Dixon for renal fat quantification. Fat quantification of the renal parenchyma using this approach proved to be impossible, yielding values ranging from negative to $>10 \%$ which is unrealistic. The unrealistic values seemed to be caused by a combination of low fat content and water-fat interference on the border of the kidney (see supplementary materials Figure S3). Furthermore, the quantitative Dixon acquisition that was used in the current study has been developed for liver fat quantification and is therefore based on the liver fat spectrum. Ideally, a renal fat spectrum should be used for the quantification, although its influence on the fat quantification is expected to be limited.

Recently, recommendation papers have been published for renal BOLD, ASL, DWI and relaxometry. ${ }^{(42,45-47)}$ Despite the current study being finished before publication of these papers, acquisition and processing has largely been performed in accordance with those recommendations. The main deviation from the recommendations is the $\mathrm{T}_{1}$ mapping, which was performed using inversion recovery with a cycled multi-slice 2D readout, in contrast to the recommended MOLLI scheme. ${ }^{(47)}$ However, in the recommendation paper it was noted that a MOLLI scheme is not developed for renal imaging and has limited spatial resolution. Furthermore, with a MOLLI scheme an apparent $T_{1}$ is measured, which is not the case for our approach. For FAIR-ASL, instead of a spin-echo EPI readout, a gradient echo EPI readout was used which was in our experience less affected by artifacts. ${ }^{(42)}$ For BOLD MRI, fasting was recommended, which was not performed in this study. ${ }^{(45)}$

Most subjects were capable of paced breathing during the ASL and T1 acquisitions. One subject fell asleep during the scan and could not easily be awakened, resulting in severe motion artifacts on the breath-hold scans. While motion artifacts were absent on all free breathing acquisitions, breath-hold acquisitions were regularly affected by either motion or inconsistent breath-holds, resulting in incomplete multiparametric datasets in 2 subjects. This was the case while breath-hold duration was less than 10s. In patients, breath-holding can be even more problematic. Therefore, robust, free-breathing acquisitions are preferred. 
Alternatively, radial acquisitions can be employed as we did for the DCE MRI. Motion artifacts in the DCE datasets, which were acquired in free breathing with radial sampling, were virtually absent. 2DPC was susceptible to acquisition errors, which led to incomplete multiparametric datasets in 3 subjects. This was partly due to the learning curve of the operators, illustrated by the fact that 2DPC acquisition in one of both renal arteries failed in the first two subjects.

\section{Limitations}

A limitation of the study is that blood samples were only taken during one visit. Therefore, we could not correct for physiological variation in kidney function. Diet and water intake were loosely controlled to be within normal ranges, but exercise and smoking were not. Therefore, the physiological state of subjects might have differed between the scans. However, the influence of those factors on measurement of MRI parameters is uncertain and normal hydration status was specifically recommended for BOLD, DWI, ASL and relaxometry. ${ }^{(42,45-47)}$ Another limitation is the relatively short time between the scan sessions (4-14 days, median 7). Based on the pharmacokinetics reported in the summary of product characteristics ${ }^{(48)}$ we expected the contrast agent to have already been eliminated during the second scan session. Surprisingly, the second $\mathrm{T}_{1}$ measurement both in cortex and medulla was significantly lower, presumably due to remaining contrast agent. ${ }^{(33)}$ Based on the measured $\mathrm{T}_{1}$ difference we can conclude that the contrast agent concentration during the second scan session $(\sim 4-8 \mathrm{nmol} / \mathrm{g}$ tissue) was too low to affect other measurements. Other measurements indeed did not show significant bias between the first and the second imaging session. ASL and DCE perfusion measurements, which directly depend on $T_{1}$, were corrected for the $T_{1}$ measured during the same session. Therefore, we expect the influence of the remaining contrast agent to be limited to the $\mathrm{T}_{1}$ measurements.

\section{Conclusion}

Various measures of renal structure and function were obtained within an acceptable acquisition time of one hour. Repeatability of all measures except for the perfusion fraction of the IVIM analysis was comparable with other tests of renal function. Furthermore, we compared various perfusion measurements in terms of repeatability and can conclude that either FAIR-ASL or 2D phase contrast can be used for renal perfusion measurements, while DCE is less reliable for perfusion analysis.

\section{Acknowledgements}

The authors would like to thank Tobias T. Pieters for his assistance with the data acquisition. 


\section{References}

1. Selby NM, Blankestijn PJ, Boor P, et al. Magnetic resonance imaging biomarkers for chronic kidney disease: a position paper from the European Cooperation in Science and Technology Action PARENCHIMA. Nephrol Dial Transplant 2018;33:ii4-ii14.

2. Friedli I, Crowe LA, Berchtold L, et al. New Magnetic Resonance Imaging Index for Renal Fibrosis Assessment: A Comparison between Diffusion-Weighted Imaging and T1 Mapping with Histological Validation. Sci Rep 2016;6:30088.

3. Caroli A, Schneider M, Friedli I, et al. Diffusion-weighted magnetic resonance imaging to assess diffuse renal pathology: a systematic review and statement paper. Nephrol Dial Transplant 2018;33:ii29-ii40.

4. Spieker M, Katsianos E, Gastl M, et al. T2 mapping cardiovascular magnetic resonance identifies the presence of myocardial inflammation in patients with dilated cardiomyopathy as compared to endomyocardial biopsy. Eur Heart J Cardiovasc Imaging 2018;19:574-582.

5. Di Cesare E. MRI of the cardiomyopathies. Eur J Radiol 2001;38:179-184.

6. Odudu A, Nery F, Harteveld AA, et al. Arterial spin labelling MRI to measure renal perfusion: a systematic review and statement paper. Nephrol Dial Transplant 2018;33:ii15-ii21.

7. Pruijm M, Mendichovszky IA, Liss P, et al. Renal blood oxygenation level-dependent magnetic resonance imaging to measure renal tissue oxygenation: a statement paper and systematic review. Nephrol Dial Transplant 2018;33:ii22-ii28.

8. Wolf M, de Boer A, Sharma K, et al. Magnetic resonance imaging T1- and T2-mapping to assess renal structure and function: a systematic review and statement paper. Nephrol Dial Transplant 2018;33:ii41-ii5o.

9. Zhang JL, Storey P, Rusinek H, et al. Reproducibility of R2* and R2 measurements in human kidneys. In: Proceedings of the 19th Annual Meeting of ISMRM, Montréal, 2011. (abstract 2954).

10. Spit KA, Muskiet MHA, Tonneijck L, et al. Renal sinus fat and renal hemodynamics: a cross-sectional analysis. Magn Reson Mater Phy 2020;33:73-80.

11. Tofts PS, Cutajar M, Mendichovszky IA, Peters AM, Gordon I. Precise measurement of renal filtration and vascular parameters using a two-compartment model for dynamic contrast-enhanced MRI of the kidney gives realistic normal values. Eur Radiol 2012;22:1320-1330.

12. Inker LA, Eckfeldt J, Levey AS, et al. Expressing the CKD-EPI (Chronic Kidney Disease Epidemiology Collaboration) cystatin C equations for estimating GFR with standardized serum cystatin C values. Am J Kidney Dis 2011;58:682-684.

13. Harteveld AA, de Boer A, Franklin SL, Leiner T, van Stralen M, Bos C. Comparison of multi-delay FAIR and pCASL labeling approaches for renal perfusion quantification at 3T MRI. Magn Reson Mater Phy 2020;33:81-94. 
14. Cox RW, Ashburner J, Breman H, et al. A (sort of) new image data format standard: NiFTI-1. In: 10th Annual Meeting Of Organisation of Human Brain Mapping, Budapest, 2004.

15. Stemkens B, Prins FM, Bruijnen T, et al. A dual-purpose MRI acquisition to combine 4D-MRI and dynamic contrast-enhanced imaging for abdominal radiotherapy planning. Phys Med Biol 2019;64:06nto2.

16. Klein S, Staring M, Murphy K, Viergever MA, Pluim JP. elastix: a toolbox for intensitybased medical image registration. IEEE Trans Med Imaging 2010;29:196-205.

17. Shamonin DP, Bron EE, Lelieveldt BP, Smits M, Klein S, Staring M. Fast parallel image registration on CPU and GPU for diagnostic classification of Alzheimer's disease. Front Neuroinform 2013;7:50.

18. de Boer A, Leiner T, Vink EE, Blankestijn PJ, van den Berg CAT. Modified dixonbased renal dynamic contrast-enhanced MRI facilitates automated registration and perfusion analysis. Magn Reson Med 2018;80:66-76.

19. Yushkevich PA, Piven J, Hazlett HC, et al. User-guided 3 D active contour segmentation of anatomical structures: significantly improved efficiency and reliability. Neuroimage 2006;31:1116-1128.

20. van Baalen S, Leemans A, Dik P, Lilien MR, Ten Haken B, Froeling M. Intravoxel incoherent motion modeling in the kidneys: Comparison of mono-, bi-, and triexponential fit. J Magn Reson Imaging 2017;46:228-239.

21. Annet L, Hermoye L, Peeters F, Jamar F, Dehoux JP, Van Beers BE. Glomerular filtration rate: assessment with dynamic contrast-enhanced MRI and a corticalcompartment model in the rabbit kidney. J Magn Reson Imaging 2004;20:843-849.

22. Sourbron SP, Michaely HJ, Reiser MF, Schoenberg SO. MRI-measurement of perfusion and glomerular filtration in the human kidney with a separable compartment model. Invest Radiol 2008;43:40-48.

23. Kukuk GM, Hittatiya K, Sprinkart AM, et al. Comparison between modified Dixon MRI techniques, MR spectroscopic relaxometry, and different histologic quantification methods in the assessment of hepatic steatosis. Eur Radiol 2015;25:2869-2879.

24. Hyslop NP, White WH. Estimating precision using duplicate measurements. J Air Waste Manag Assoc 2009;59:1032-1039.

25. Martin Bland J, Altman D. STATISTICAL METHODS FOR ASSESSING AGREEMENT BETWEEN TWO METHODS OF CLINICAL MEASUREMENT. The Lancet 1986;327:307-310.

26. R Development Core Team. R: A language and environment for statistical computing. R Foundation for Statistical Computing. Vienna, Austria: R Foundation for Statistical Computing; 2018.

27. Toffaletti JG, McDonnell EH. Variation of serum creatinine, cystatin C, and creatinine clearance tests in persons with normal renal function. Clin Chim Acta 2008;395:115119. 
28. Florijn KW, Barendregt JN, Lentjes EG, et al. Glomerular filtration rate measurement by "single-shot" injection of inulin. Kidney Int 1994;46:252-259.

29. Cox EF, Buchanan CE, Bradley CR, et al. Multiparametric Renal Magnetic Resonance Imaging: Validation, Interventions, and Alterations in Chronic Kidney Disease. Front Physiol 2017;8:696.

30. Buchanan CE, Mahmoud H, Cox EF, et al. Quantitative assessment of renal structural and functional changes in chronic kidney disease using multi-parametric magnetic resonance imaging. Nephrol Dial Transplant 2019; in press. doi: 10.1093/ndt/gfz129.

31. Bane O, Hectors SJ, Gordic S, et al. Multiparametric magnetic resonance imaging shows promising results to assess renal transplant dysfunction with fibrosis. Kidney Int 2020;97:414-420.

32. Dekkers IA, Paiman EHM, de Vries APJ, Lamb HJ. Reproducibility of native T1 mapping for renal tissue characterization at 3T. J Magn Reson Imaging 2019;49:588596.

33. de Boer A, Harteveld AA, Pieters TT, et al. Decreased native renal T1 up to one week after gadobutrol administration in healthy volunteers. J Magn Reson Imaging 2019; in press. doi: 10.1002/jmri.27014.

34. Simon-Zoula SC, Hofmann L, Giger A, et al. Non-invasive monitoring of renal oxygenation using BOLD-MRI: a reproducibility study. NMR Biomed 2006;19:84-89.

35. Khatir DS, Pedersen M, Jespersen B, Buus NH. Reproducibility of MRI renal artery blood flow and BOLD measurements in patients with chronic kidney disease and healthy controls. J Magn Reson Imaging 2014;40:1091-1098.

36. Kline TL, Edwards ME, Garg I, et al. Quantitative MRI of kidneys in renal disease. Abdom Radiol (NY) 2018;43:629-638.

37. Bane O, Wagner M, Zhang JL, et al. Assessment of renal function using intravoxel incoherent motion diffusion-weighted imaging and dynamic contrast-enhanced MRI. J Magn Reson Imaging 2016;44:317-326.

38. Pan J, Zhang H, Man F, et al. Measurement and scan reproducibility of parameters of intravoxel incoherent motion in renal tumor and normal renal parenchyma: a preliminary research at 3.0 T MR. Abdom Radiol (NY) 2018;43:1739-1748.

39. Koopman MG, Koomen GC, Krediet RT, de Moor EA, Hoek FJ, Arisz L. Circadian rhythm of glomerular filtration rate in normal individuals. Clin Sci (Lond) 1989;77:105111.

40. Johnson S, Rishi R, Andone A, et al. Determinants and functional significance of renal parenchymal volume in adults. Clin J Am Soc Nephrol 2011;6:70-76.

41. Cutajar M, Thomas DL, Hales PW, Banks T, Clark CA, Gordon I. Comparison of ASL and DCE MRI for the non-invasive measurement of renal blood flow: quantification and reproducibility. Eur Radiol 2014;24:1300-1308.

42. Nery F, Buchanan CE, Harteveld AA, et al. Consensus-based technical recommendations for clinical translation of renal ASL MRI. Magn Reson Mater Phy 2020;33:141-161. 
43. Eikefjord E, Andersen E, Hodneland E, et al. Dynamic contrast-enhanced MRI measurement of renal function in healthy participants. Acta Radiol 2017;58:748-757.

44. Cutajar M, Mendichovszky IA, Tofts PS, Gordon I. The importance of AIF ROI selection in DCE-MRI renography: reproducibility and variability of renal perfusion and filtration. Eur J Radiol 2010;74:e154-16o.

45. BaneO, Mendichovszky IA, Milani B, etal. Consensus-based technical recommendations for clinical translation of renal BOLD MRI. Magn Reson Mater Phy 2020;33:199-215.

46. Ljimani A, Caroli A, Laustsen C, et al. Consensus-based technical recommendations for clinical translation of renal diffusion-weighted MRI. Magn Reson Mater Phy 2020;33:177-195.

47. Dekkers IA, de Boer A, Sharma K, et al. Consensus-based technical recommendations for clinical translation of renal T1 and T2 mapping MRI. Magn Reson Mater Phy 2020;33:163-176.

48. Bayer Healthcare Pharmaceuticals. HIGHLIGHTS OF PRESCRIBING INFORMATION- GADAVIST (gadobutrol) injection, for intravenous use. US Food and Drug Administration; 2011.

49. Ordidge RJ, Gibbs P, Chapman B, Stehling MK, Mansfield P. High-speed multislice T1 mapping using inversion-recovery echo-planar imaging. Magn Reson Med 1990;16:238-245. 


\section{Supplementary Materials}

\section{Supplementary Methods}

\section{Segmentation}

Segmentation was performed twice: first a rough, wide mask around the kidney was generated to aid the registration, followed by the generation of separate masks containing either the entire cortex or the medulla to enable ROI analysis. The approach to the generation of the masks is explained below.

\section{DCE, qDixon, R2* Mapping/BOLD, T2 Mapping and DWI}

For DCE, quantitative Dixon, $\mathrm{R}_{2}{ }^{*}$ mapping/BOLD, $\mathrm{T}_{2}$ mapping and DWI both the initial rough masks and the cortex and medulla masks were generated on the DCE data using k-means clustering followed by dilation of the mask as described before. (1) If DCE data was not available, the BOLD images were used.

The masks were transferred from the DCE image to the image of interest transforming the masks from one image-space to the other. This was done using a transformation matrix which was obtained using (deformable) image registration. Image registration was performed on median images. The source images were generally stored in $4 \mathrm{D}$ matrices, with the timepoints (images corresponding to different echotimes, inversion times, b-values etc.) in the $4^{\text {th }}$ dimension. To obtain a median image, the median over the $4^{\text {th }}$ dimension was taken. Median images were generally sharper than mean images and therefore more suitable to use for image registration.

To transform the masks, the following steps were carried out:

1. Compute median image of DCE image

2. Register median DCE image to anatomical Dixon using deformable registration in Elastix (version 4.9.0 ${ }^{(2,3)}$ )

3. Transform mask from DCE to anatomical Dixon

4. (Only for cortex/medulla masks) If necessary: manually adapt mask (by author with 5 years of experience in renal imaging, A.B.)

5. Compute median image of image of interest (for example $T_{2}$ mapping source data)

6. Register anatomical image to median image using Elastix (for example median image of $\mathrm{T}_{2}$ mapping source data)

7. Transform mask from anatomical space to image of interest

8. (Only for cortex/medulla masks) If necessary: manually adapt mask using the image of interest as a reference. 
For the rough initial mask, median images of the unregistered source images were used for registration, while for the cortex and medulla masks median images of the registered source images were used.

The stepwise approach explained above worked well for the $\mathrm{R}_{2}{ }^{*}$ mapping/ BOLD and quantitative Dixon images (both fast gradient echo readouts), but for the DWI and $\mathrm{T}_{2}$ mapping images manual refinement was often required. This was mainly due to the EPI readouts used for both $\mathrm{T}_{2}$ mapping and DWI, which were deformed due to Bo inhomogeneities. This resulted in lengthening of the kidneys, which made proper registration challenging.

For some examples of the cortex and medulla masks, see figure S1.

For DCE MRI, also an ROI in the aorta had to be defined for measurement of the arterial input function (AIF). Because of large variations of the AIF within the aorta, we decided to use large ROIs covering most of the caudal part of the aorta within the imaging slab. The cranial part of the aorta was not included because of inflow artifacts.

\section{D Phase Contrast}

For 2D phase contrast, a mask of the renal artery had to be generated. Again, first a wide mask had to be generated to aid registration. This was achieved by thresholding on a magnitude image followed by a dilation of 8 voxels. After registration, a precise mask was generated in two steps. First a mask was created by thresholding on a magnitude image, followed by 2 voxel dilation. Next, the time-intensity curves of all voxels within the mask were calculated. The mean flow and the standard deviation of the mean velocity curve was calculated. In the final mask, Only voxels with a flow of at least half the mean flow and a standard deviation of less than twice the standard deviation of the mean velocity curve were included. This ensured that stationary voxels were not included (low mean velocity) and that voxels containing noise or registration errors (resulting in high standard-deviations) were excluded. Using the resulting mask, the vessel area and the total flux through the vessel could be calculated.

\section{T, Maps and ASL}

For $\mathrm{T}_{1}$ maps, FAIR-ASL and $\mathrm{M}_{\mathrm{o}}$ images, masks were generated using k-means clustering on the $\mathrm{T}_{1}$ source images. Since these images were all acquired using the same geometry settings and registered together, the resulting mask could simply be copied to the other images. 


\section{Quality Assessment}

The following criteria were used to decide if data had to be excluded:

1. Failure of the acquisition

a. Reasons differ per sequence, including problems with intravenous access for DCE MRI, erroneous planning for 2D phase contrast and FAIR MRI, erroneous cardiac triggering for $2 \mathrm{D}$ phase contrast

2. Presence of artifacts affecting more than halve of the kidney. Artifacts include

a. Susceptibility artifacts $\left(\mathrm{T}_{2}{ }^{*}\right.$ mapping)

b. Motion artifacts (all breathheld acquisitions and T1 mapping)

c. Flow artifacts

d. Streaking artifacts (radial acquisition (DCE))

e. Fat-water cancellation artifacts (for in-phase acquisitions including (quantitative) Dixon and $\mathrm{T}_{2}{ }^{*}$ mapping)

f. Banding artifacts ( $\mathrm{T}_{2}$ mapping)

g. Insufficient fat suppression ( $\mathrm{T}_{2}$ mapping)

h. Fold-over artifacts (spin echo EPI readouts with feet-head phase encoding)

i. Gibbs ringing at water-fat boundary (quantitative Dixon)

If either (1) or (2) was present, data belonging to the affected kidney and the affected sequence were discarded.

\section{Model Fitting}

Relaxometry

$\mathrm{T}_{1}$ data was fitted to the following model:

$$
S=\left|S_{0}\left(1-2 e^{-T I / T_{1}}\right)\right|
$$

Here $S_{o}$ denotes the signal without weighting and $T_{I}$ is the inversion time. For the T1 series, the point closest to the zero crossing was deleted because of low signal-tonoise ratio.

$\mathrm{T}_{2}$ data was fitted to the following model:

$$
S=S_{0} \mathrm{e}^{-T E / T_{2}}
$$

Where TE denotes the echo time. And $\mathrm{R}_{2}{ }^{*}$ data was fitted to the following model:

$$
S=S_{0} e^{-T E R_{2}^{*}}
$$




\section{DCE MRI}

DCE modelling required measurement of an arterial input function (AIF) in the feeding artery (in this case the abdominal aorta) denoted $\mathrm{C}_{\mathrm{A}}(\mathrm{t}$ ) and the time intensity curves in the renal parenchyma denoted $\mathrm{C}(\mathrm{t})$. The AIF is dispersed with a vascular input response function (VIRF) to account for dispersion of the contrast bolus in the renal vascular bed.

For modelling of the DCE data, a two-compartment model was used consisting of a tubular and a plasma (vascular) compartment ${ }^{(4)}$ which is described by the following equations:

$$
\begin{gathered}
\operatorname{VIRF}(t)=0 \quad(t<\text { delay }) \\
\operatorname{VIRF}(t)=\frac{1}{T_{P}} \exp \left(-\frac{t-\text { delay }}{T_{P}}\right)(t \geq \text { delay }) \\
C_{P}(t)=\operatorname{VIRF} \times C_{A}(t) \\
C(t)=V_{P} C_{P}(t)+F_{T} e^{-\frac{t}{T_{T}}} \times C_{P}(t)
\end{gathered}
$$

The delay is the delay before the tracer appears in the renal vascular bed and $T_{\mathrm{P}}$ defines the broadening of the tracer bolus while travelling the vascular bed. $\mathrm{T}_{\mathrm{P}}+$ delay equals the mean residence time (MRT). ${ }^{(5)} \mathrm{C}_{\mathrm{P}}$ is the contrast agent concentration in the plasma compartment. $\left.\mathrm{V}_{\mathrm{P}}\right\urcorner$ is the fractional volume of the plasma compartment. $\mathrm{VP} / \mathrm{MRT}$ gives $\mathrm{F}_{\mathrm{P}}$, the (plasma) perfusion per unit of tissue volume. $\mathrm{F}_{\mathrm{T}}$ is the tubular flow or the GFR per unit of tissue volume. $\mathrm{T}_{\mathrm{T}}$ is the transit time of the contrast agent through the tubular compartment.

The parameters in this model can be described as vascular $\left(\mathrm{T}_{\mathrm{p}}, \mathrm{V}_{\mathrm{p}}\right.$ and delay) and tubular $\left(\mathrm{F}_{\mathrm{T}}\right.$ and $\left.\mathrm{T}_{\mathrm{T}}\right)$. The vascular parameters mostly depend on the first part of the time intensity curve, while the tubular parameters are defined better by the second part of the curve. This is illustrated by the difference in magnitude between $\mathrm{T}_{\mathrm{P}}$ and delay (a few seconds) and $\mathrm{T}_{\mathrm{T}}$, which is around two orders of magnitude larger. Therefore, this model was fitted in a two-step approach:

1. Fit all parameters, but put double weight on the second half of the time-intensity curve, compared to 1 on the first half. Save $F_{T}$ and $T_{T}$, discard all vascular parameters.

2. Fix $\mathrm{F}_{\mathrm{T}}$ and $\mathrm{T}_{\mathrm{T}}$ on the values obtained in step one. Fit $\mathrm{T}_{\mathrm{D}}$ and $\mathrm{V}_{\mathrm{P}}$ on only the first 50 (out of 80 ) points of the time intensity curve. Loop over a range of delay values to obtain the delay for which the fit is best. 


\section{References}

1. de Boer A, Leiner T, Vink EE, Blankestijn PJ, van den Berg CAT. Modified dixonbased renal dynamic contrast-enhanced MRI facilitates automated registration and perfusion analysis. Magn Reson Med 2018;80:66-76.

2. Klein S, Staring M, Murphy K, Viergever MA, Pluim JP. elastix: a toolbox for intensitybased medical image registration. IEEE Trans Med Imaging 2010;29:196-205.

3. Shamonin DP, Bron EE, Lelieveldt BP, Smits M, Klein S, Staring M. Fast parallel image registration on CPU and GPU for diagnostic classification of Alzheimer's disease. Front Neuroinform 2013;7:50.

4. Annet L, Hermoye L, Peeters F, Jamar F, Dehoux JP, Van Beers BE. Glomerular filtration rate: assessment with dynamic contrast-enhanced MRI and a corticalcompartment model in the rabbit kidney. J Magn Reson Imaging 2004;20:843-849.

5. Tofts PS, Cutajar M, Mendichovszky IA, Peters AM, Gordon I. Precise measurement of renal filtration and vascular parameters using a two-compartment model for dynamic contrast-enhanced MRI of the kidney gives realistic normal values. Eur Radiol 2012;22:1320-1330.

6. Cox EF, Buchanan CE, Bradley CR, et al. Multiparametric Renal Magnetic Resonance Imaging: Validation, Interventions, and Alterations in Chronic Kidney Disease. Front Physiol 2017;8:696.

7. Buchanan CE, Mahmoud H, Cox EF, et al. Quantitative assessment of renal structural and functional changes in chronic kidney disease using multi-parametric magnetic resonance imaging. Nephrol Dial Transplant 2019; in press. doi: 10.1093/ndt/gfz129.

8. Zhang JL, Storey P, Rusinek H, et al. Reproducibility of R2* and R2 measurements in human kidneys. In: Proceedings of the 19th Annual Meeting of ISMRM, Montréal, 2011. (abstract 2954).

9. Simon-Zoula SC, Hofmann L, Giger A, et al. Non-invasive monitoring of renal oxygenation using BOLD-MRI: a reproducibility study. NMR Biomed 2006;19:84-89.

10. Khatir DS, Pedersen M, Jespersen B, Buus NH. Reproducibility of MRI renal artery blood flow and BOLD measurements in patients with chronic kidney disease and healthy controls. J Magn Reson Imaging 2014;40:1091-1098.

11. Kline TL, Edwards ME, Garg I, et al. Quantitative MRI of kidneys in renal disease. Abdom Radiol (NY) 2018;43:629-638.

12. Bane O, Hectors SJ, Gordic S, et al. Multiparametric magnetic resonance imaging shows promising results to assess renal transplant dysfunction with fibrosis. Kidney Int 2020;97:414-420.

13. Borrelli P, Cavaliere C, Basso L, Soricelli A, Salvatore M, Aiello M. Diffusion Tensor Imaging of the Kidney: Design and Evaluation of a Reliable Processing Pipeline. Sci Rep 2019;9:12789. 
14. Notohamiprodjo M, Dietrich O, Horger W, et al. Diffusion tensor imaging (DTI) of the kidney at 3 tesla-feasibility, protocol evaluation and comparison to 1.5 Tesla. Invest Radiol 2010;45:245-254.

15. Cutajar M, Clayden JD, Clark CA, Gordon I. Test-retest reliability and repeatability of renal diffusion tensor MRI in healthy subjects. Eur J Radiol 2011;80:e263-268.

16. Bane O, Wagner M, Zhang JL, et al. Assessment of renal function using intravoxel incoherent motion diffusion-weighted imaging and dynamic contrast-enhanced MRI. J Magn Reson Imaging 2016;44:317-326.

17. Pan J, Zhang H, Man F, et al. Measurement and scan reproducibility of parameters of intravoxel incoherent motion in renal tumor and normal renal parenchyma: a preliminary research at 3.0 T MR. Abdom Radiol (NY) 2018;43:1739-1748.

18. Hammon M, Janka R, Siegl C, et al. Reproducibility of Kidney Perfusion Measurements With Arterial Spin Labeling at 1.5 Tesla MRI Combined With Semiautomatic Segmentation for Differential Cortical and Medullary Assessment. Medicine (Baltimore) 2016;95:e3083.

19. Cutajar M, Thomas DL, Hales PW, Banks T, Clark CA, Gordon I. Comparison of ASL and DCE MRI for the non-invasive measurement of renal blood flow: quantification and reproducibility. Eur Radiol 2014;24:1300-1308.

20. Gillis KA, McComb C, Foster JE, et al. Inter-study reproducibility of arterial spin labelling magnetic resonance imaging for measurement of renal perfusion in healthy volunteers at 3 Tesla. BMC Nephrol 2014;15:23.

21. Artz NS, Sadowski EA, Wentland AL, et al. Reproducibility of renal perfusion MR imaging in native and transplanted kidneys using non-contrast arterial spin labeling. $\mathrm{J}$ Magn Reson Imaging 2011;33:1414-1421.

22. Karger N, Biederer J, Lusse S, et al. Quantitation of renal perfusion using arterial spin labeling with FAIR-UFLARE. Magn Reson Imaging 2000;18:641-647.

23. Eikefjord E, Andersen E, Hodneland E, et al. Dynamic contrast-enhanced MRI measurement of renal function in healthy participants. Acta Radiol 2017;58:748-757.

24. Cutajar M, Mendichovszky IA, Tofts PS, Gordon I. The importance of AIF ROI selection in DCE-MRI renography: reproducibility and variability of renal perfusion and filtration. Eur J Radiol 2010;74:e154-160.

25. Bax L, Bakker CJ, Klein WM, Blanken N, Beutler JJ, Mali WP. Renal blood flow measurements with use of phase-contrast magnetic resonance imaging: normal values and reproducibility. J Vasc Interv Radiol 2005;16:807-814. 


\section{Supplementary Figures}

Supplementary Table 1: Comparison of CoVs measured in this study to literature values. For literature values, only inter-session repeatability with comparable scan protocols were considered, unless stated otherwise. Literature values are only reported for comparable scan protocols and inter-session repeatability, unless stated otherwise.

\begin{tabular}{|c|c|c|c|c|}
\hline \multirow[b]{2}{*}{ Sequence } & \multirow[b]{2}{*}{ Parameter } & \multirow[b]{2}{*}{$\operatorname{CoV}(\%)$} & \multicolumn{2}{|c|}{ Literature } \\
\hline & & & CoV (\%) & References \\
\hline \multicolumn{5}{|c|}{ Relaxometry } \\
\hline $\mathrm{T}_{1}$ map & $\begin{array}{l}\mathrm{T}_{1} \text { cortex } \\
\mathrm{T}_{1} \text { medulla }\end{array}$ & $\begin{array}{l}5.1 \\
2.8\end{array}$ & $\begin{array}{l}2.0-2.9 \% \\
1.8-3.9 \%\end{array}$ & $\begin{array}{l}(6,7) \\
(6,7)\end{array}$ \\
\hline $\mathrm{T}_{2} \operatorname{map}$ & $\mathrm{T}_{2}$ parenchyma & 2.9 & $3 \cdot 4-3.9 \% *$ & (8) \\
\hline $\mathrm{R}_{2}^{*} \operatorname{map}$ & $\begin{array}{l}\mathrm{R}_{2}^{*} \text { cortex } \\
\mathrm{R}_{2}^{*} \text { medulla }\end{array}$ & $\begin{array}{l}6.1 \\
5.8 \\
\end{array}$ & $\begin{array}{l}3.6-14 \% \\
3.0-12 \% \\
\end{array}$ & $\begin{array}{c}(6,7,9-12) \\
(7,9-12) \\
\end{array}$ \\
\hline \multicolumn{5}{|c|}{ DTI and IVIM } \\
\hline DTI & $\begin{array}{l}\text { MD cortex } \\
\text { MD medulla } \\
\text { FA cortex } \\
\text { FA medulla }\end{array}$ & $\begin{array}{l}3.7 \\
2.8 \\
6.7 \\
5.2\end{array}$ & $\begin{array}{c}1.1-2.0 \% \ddagger \\
1.3-5.6 \% \neq \\
4.7-27.3 \% \neq \\
4.4-11.4 \% \neq\end{array}$ & $\begin{array}{l}(12-15) \\
(12-15) \\
(12-15) \\
(12-15)\end{array}$ \\
\hline IVIM & $\begin{array}{l}\mathrm{D} \text { cortex } \\
\mathrm{D} \text { medulla } \\
\mathrm{F}_{\mathrm{P}} \text { cortex } \\
\mathrm{F}_{\mathrm{P}} \text { medulla }\end{array}$ & $\begin{array}{l}6.7 \\
7.2 \\
24 \\
18\end{array}$ & $\begin{array}{c}5 \cdot 9-14 \% \\
5 \cdot 8-22 \% \\
14-22 \% \\
22-54 \% \\
\end{array}$ & $\begin{array}{c}(6,7,11,12,16,17) \\
(7,12,16,17) \\
(6,12,16,17) \\
(12,16,17) \\
\end{array}$ \\
\hline \multicolumn{5}{|c|}{ Perfusion and GFR } \\
\hline FAIR & $\begin{array}{l}\text { Cortical perfusion } \\
\text { ATT }\end{array}$ & $\begin{array}{l}10 \\
12\end{array}$ & $\begin{array}{c}6.4-28 \% \\
-\end{array}$ & $\begin{array}{c}(6,7,18-22) \\
-\end{array}$ \\
\hline DCE & $\begin{array}{l}\text { Parenchymal perfusion } \\
\text { MRT } \\
\text { skGFR }\end{array}$ & $\begin{array}{l}16 \\
12 \\
13\end{array}$ & $\begin{array}{c}14-29.5 \% \\
7-16 \% \\
17-22 \%\end{array}$ & $\begin{array}{c}(5,16,19,23,24) \\
(5,16) \\
(16,23)\end{array}$ \\
\hline $\mathrm{PC}$ & RBF per artery & 13 & $10-26 \%$ & $(6,7,11,25)$ \\
\hline \multicolumn{5}{|c|}{ Fat quantification } \\
\hline Dixon & $\begin{array}{l}\text { Fat fraction sinus } \\
\text { Fat volume sinus }\end{array}$ & $\begin{array}{l}9.2 \\
9.5 \\
\end{array}$ & - & - \\
\hline
\end{tabular}

CoV: within subject coefficient of variation; DTI: diffusion tensor imaging; MD: mean diffusivity; FA: fractional anisotropy; IVIM: intravoxel incoherent motion; D: diffusion coefficient; Fp: perfusion fraction; FAIR: flow alternating inversion recovery; ATT: arterial transit time; DCE: dynamic contrast enhanced MRI; skGFR: single kidney glomerular filtration rate; MRT: mean residence time; PC: phase contrast; RBF: renal blood flow; $t$ : absolute relative difference: $¥$ : intra-session repeatability (two consecutive scans with replanning). 
BOLD

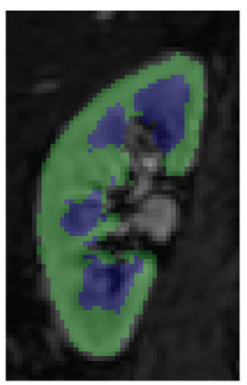

DTI

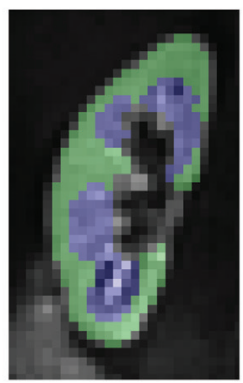

$T_{1} \operatorname{map}$

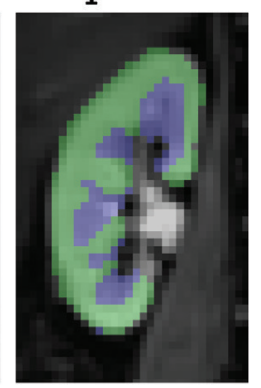

DCE

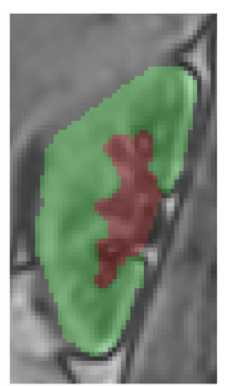

Figure S1. Examples of cortex and medulla masks for different readouts. Also the sinus mask belonging to the quantitative Dixon is shown. Note that the DCE image is acquired in transverse direction.
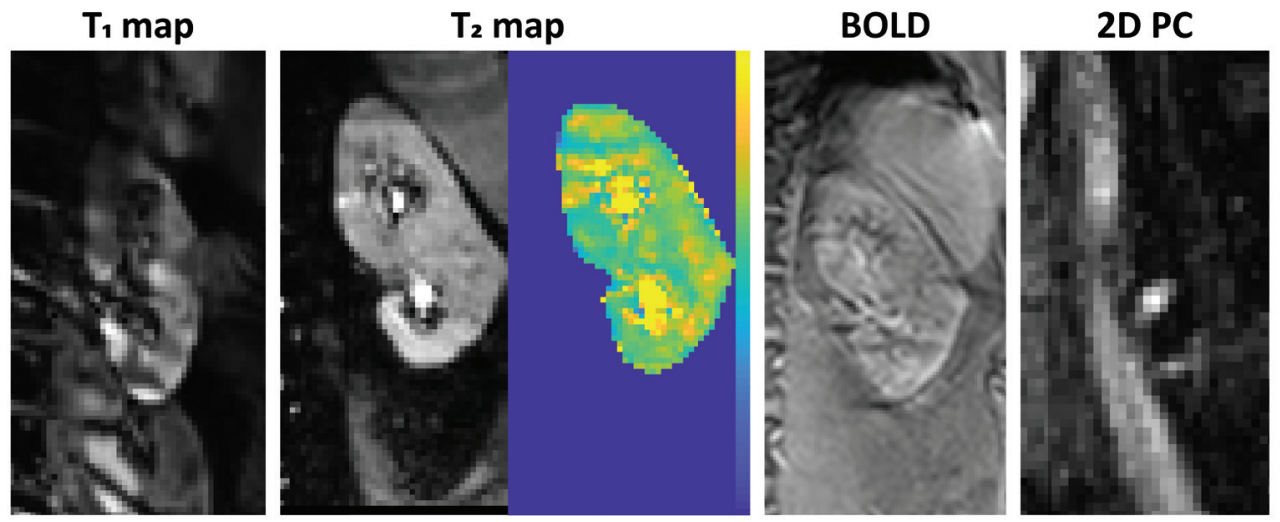

Figure S2. Examples of excluded exams. The $\mathrm{T}_{1}$ map was affected by motion. In the $\mathrm{T}_{2}$ map banding artifacts are visible, as well as artifacts due to insufficient fat suppression. The BOLD map was affected by motion. The 2D PC exam was erroneously planned (not perpendicular to the vessel, as illustrated by the elliptical shape of the vessel cross-section).

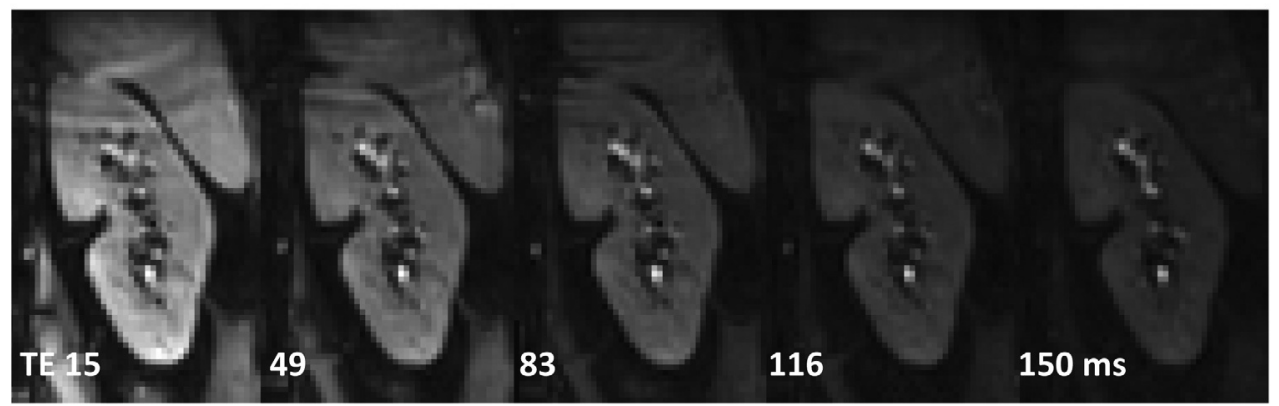

Figure S3. Example of banding artifacts on the $\mathrm{T}_{2}$ source images. The bands shift in location depending on the preparation duration, shifting up with increasing duration. They are probably caused by $B_{o}$ inhomogeneities outside the field of view, resulting in high-intensity bands folding in. 


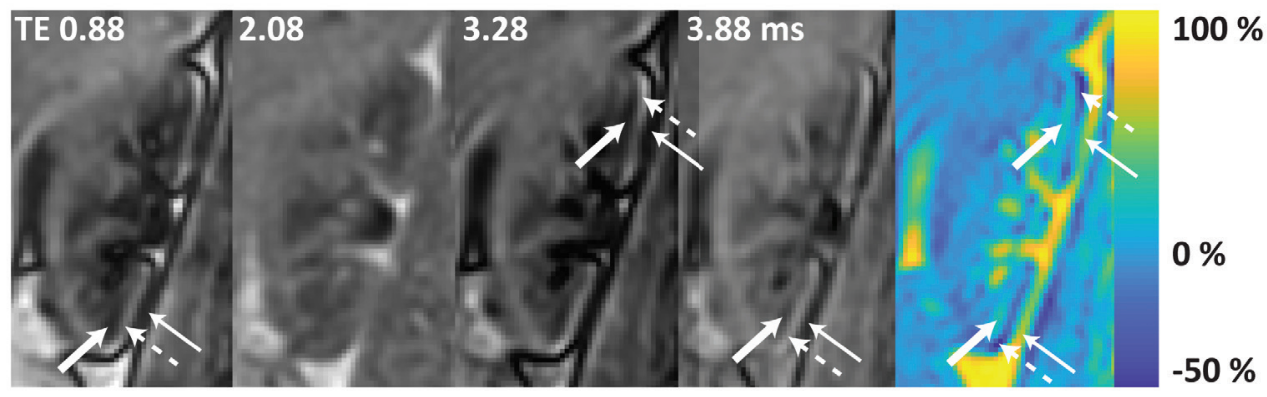

Figure S4. example of artifacts due to water-fat interference on the quantitative Dixon images. The thin arrow is a water-fat cancellation artifact, the dashed and fat arrow point at high and low intensity bands in the renal parenchyma, probably due to a Gibbs ringing artifact. 



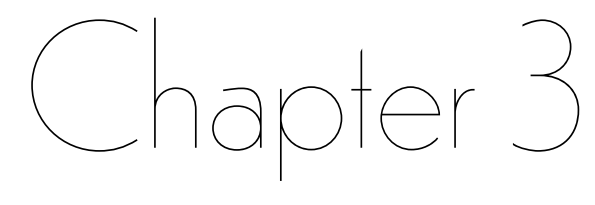

\section{Moditied Dixon Based Renal Dynamic Contrast Enhanced MRI Facilitates Automated Registration and Perfusion}

$$
C(t)=V_{P} C_{P}(t)+F_{T} \int_{0}^{t} e^{-\tau / T_{T}} C_{P}(t-\tau) d \tau
$$

\section{Author contributions}

de Boer A, Leiner T, Vink EE, Blankestijn PJ, van den Berg CAT. 


\section{Abstract}

Purpose Renal dynamic contrast enhanced (DCE) MRI provides information on renal perfusion and filtration. However, clinical implementation is hampered by challenges in postprocessing as a result of misalignment of the kidneys due to respiration. We propose to perform automated image registration using the fat-only images derived from a modified Dixon reconstruction of a dual-echo acquisition because these provide consistent contrast over the dynamic series.

Methods DCE data of 10 hypertensive patients was used. Dual-echo images were acquired at $1.5 \mathrm{~T}$ with temporal resolution of $3.9 \mathrm{~s}$ during contrast agent (CA) injection. Dixon fat, water, and in-phase and opposed-phase (OP) images were reconstructed. Postprocessing was automated. Registration was performed both to fat-images and OP-images for comparison. Perfusion and filtration values were extracted from a two-compartment model fit.

Results Automatic registration to fat-images performed better than automatic registration to OP-images with visible contrast enhancement. Median vertical misalignment of the kidneys was $14 \mathrm{~mm}$ prior to registration, compared to $3 \mathrm{~mm}$ and $5 \mathrm{~mm}$ with registration to fat images and OP-images, respectively $(\mathrm{p}=0.03)$. Mean perfusion values and MR-based glomerular filtration rates (GFR) were $233 \pm 64 \mathrm{~mL} / 100 \mathrm{~mL} / \mathrm{min}$ and $60 \pm 36 \mathrm{ml} / \mathrm{min}$, respectively, based on fat-registered images. MR-based GFR correlated with creatinine-based GFR $(\mathrm{p}=0.04)$ for fatregistered images. For unregistered and OP-registered images, this correlation was not significant.

Conclusion Absence of contrast changes on Dixon fat-images improves registration in renal DCE MRI and enables automated postprocessing, resulting in a more accurate estimation of GFR. 


\section{Introduction}

Dynamic contrast enhanced (DCE) MRI of the kidneys provides information on renal perfusion ${ }^{(1)}$ and (single kidney) glomerular filtration rate ((sk)GFR) ${ }^{(2-4)}$. It can be used for characterization of renal masses ${ }^{(5-7)}$ and is a promising noninvasive tool for early detection of renal transplant rejection ${ }^{(8,9)}$, although the risk of nephrogenic systemic fibrosis might limit the use of contrast-based MR techniques in this population. DCE MRI consists of acquisition of a dynamic series of images during injection of a contrast agent (CA). By fitting a pharmacokinetic model to the data, quantitative information on renal perfusion and filtration can be obtained. Although renal DCE MRI is a growing field of research, implementation in clinical practice is limited due to challenges in the postprocessing of data and a lack of standardized protocols. A main postprocessing challenge is misalignment of the kidneys in the dynamic series due to respiration. This leads to disturbances in the time-intensity curves, which affects the pharmacokinetic model fit and therefore leads to errors in the estimation of perfusion and filtration values ${ }^{(10)}$.

Numerous methods exist to deal with respiratory motion in the kidneys, with breath holding presumably the simplest and most intuitive ${ }^{(11)}$. Yet, a single breath hold limits the length of the time series and requires cooperation of the patient, which is hardly achievable in diseased or pediatric populations. Alternatively, artifacts induced by respiratory motion can be minimized by respiratory gating ${ }^{(12,13)}$, but the resultant time intervals are not regular and temporal resolution is reduced. Therefore, a free-breathing approach with retrospective motion correction often is preferred. Approaches include retrospective respiratory gating ${ }^{(5,14)}$, that is, discarding images acquired during inspiration, and image registration. Retrospective triggering again limits temporal resolution because images acquired during inspiration are removed from the dataset. Furthermore, image registration in DCE MRI is complicated by the large dynamic range in image contrast over the dynamic series. To avoid this problem, advanced registration techniques are used, such as those based on edge detection ${ }^{(8,10,15-18)}$ or mutual information (MI) ${ }^{(19,20)}$. Registration has been shown to improve estimation of filtration ${ }^{(21,22)}$.

We propose to circumvent the problem of changes in contrast enhancement through use of a Dixon reconstruction of dual-echo DCE data, which allows for reconstruction of fat-only images on which renal contours are intrinsically outlined. Because gadolinium based CAs are confined to the intravascular and extracellular compartments ${ }^{(23)}$ kidney outlines are not subject to changes typically seen over the course of the DCE acquisition using conventional sequences.

Furthermore, we aim to enable automated registration and perfusion analysis of renal DCE data, by implementing a combination of image registration to Dixon fatimages and automated kidney delineation using the approach proposed by Zöllner et al. ${ }^{(19)}$. 


\section{Methods}

\section{Subjects}

A representative selection of 10 patients (4 male, mean age 57 years, mean systolic/ diastolic blood pressure $156 / 94 \mathrm{mmHg}$, mean estimated glomerular filtration rate (eGFR) $80 \mathrm{ml} / \mathrm{min} / 1.73 \mathrm{~m}^{2}$ ) was made out of a cohort of hypertensive patients referred for treatment with renal denervation. A detailed description of this population was published earlier. ${ }^{(24)}$ eGFR was estimated using the chronic kidney disease epidemiology collaboration (CKD-EPI) formula (25), based on creatinine clearance. Plasma creatinine levels were measured within a week of the MRI. All patients underwent DCE MRI as part of the renal denervation workup. Permission from the local medical ethics review committee was obtained, and all patients signed informed consent prior to inclusion into the study.

\section{Imaging Protocol}

Dual-echo images were acquired on a $1.5 \mathrm{~T}$ (Ingenia, software release 4.1, Philips Healthcare, Eindhoven, the Netherlands) MR system using a 3D gradient-echo dualecho protocol with a modified Dixon reconstruction ${ }^{(26)}$. All images were acquired with repetition time of $5.9 \mathrm{~ms}$ and echo times of 1.8 and $4.0 \mathrm{~ms}$. First, three precontrast acquisitions (prescans) with a variable flip angle $\left(5,13\right.$ and $\left.20^{\circ}\right)$ were acquired for determination of precontrast longitudinal relaxation rate $\left(\mathrm{R}_{1}\right)$. Subsequently, a dynamic series consisting of 25 dynamics with flip angle $15^{\circ}$ was acquired. Twentyfive coronal slices were acquired with voxel size $2.5 \times 2.5 \times 3.0 \mathrm{~mm}^{3}$ and field of view of $420 \times 420 \mathrm{~mm}$. Based on the work of Michaely et al. ${ }^{(12)}$ we kept temporal resolution to less than $4 \mathrm{~s}$ per dynamic phase. Using a sensitivity-encoding technique factor of 2.5 in left-right direction an acquisition time of $3.9 \mathrm{~s}$ per dynamic was achieved. During the dynamic series, $0.1 \mathrm{mmol} / \mathrm{kg}$ of Gadovist was infused at a rate of $1 \mathrm{~mL} / \mathrm{s}$, followed by a saline flush of $25 \mathrm{~mL}$. Subsequently, Dixon water-only, fat-only, in phase (IP) and opposed phase (OP) images were generated.

\section{Segmentation}

Postprocessing was performed with a MatLab (MatLab 2014b, MathWorks, Natick, Massachusetts, USA) graphic user interface that was developed in-house. Kidney delineation was performed according to the image segmentation method described by Zöllner et al. ${ }^{(19)}$. This approach consists of k-means clustering of the voxelbased time-intensity curves obtained from the whole range of dynamics. Due to the difference between cortical and medullary time-intensity curves, cortex and medulla usually are assigned to different clusters. To obtain parenchymal regions of interest (ROIs), cortical and medullary ROIs were combined. To improve speed and robustness of this method, we made some slight adjustments. First, because 
fat-images were available, voxels containing mostly fatty tissue could easily be excluded using a simple thresholding approach. In adipose subjects with enough fat surrounding the kidneys, thresholding alone was sufficient to create renal masks. In the remaining subjects, thresholding diminished the computation time because adipose tissue could be excluded for clustering. The default fat-threshold could be adjusted manually using a slider. Second, because the renal cluster often also encompassed the renal artery, masks were eroded and dilated to exclude the artery.

Due to smoother time-intensity curves after registration, the segmentation algorithm performed better on registered data. Therefore, it was performed both before and after image registration. The initial rough masks (Figure 1a) were employed to make wide crops around both kidneys to enable separate registration. The second and more precise masks (Figure 1b), was used for calculation of whole kidney timeintensity curves. For each segmentation manual interaction was required to adjust the fat threshold (optionally); set the number of clusters; and label the resultant masks as kidney, cortex, or medulla.
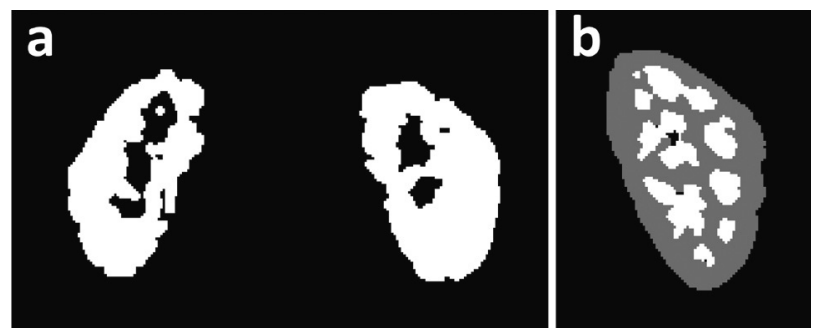

Figure 1. Segmentation of the kidney; a rough mask created before registration, used only to crop with a wide margin around the kidney; b precise cortical (grey) and medullary (white) mask created after registration. Combined, these masks form a parenchymal mask.

Segmentation of a ROI inside the aorta for determination of the arterial input function (AIF) was fully automated (Figure 2). Analogous to a maximum intensity projection (MIP), which consists of the maximum intensities reached in a spatial direction, a time MIP was created, which consists of the maximum intensities reached in the dynamic series. On this time MIP, $98 \%$ of voxels with lowest signal intensity were discarded. For the remaining voxels, components consisting of connected voxels were identified. The largest component represented the aorta. To minimize the impact of inflow often present in the cranial part of the aorta, a $5 \times 8$ voxel arterial ROI was placed in the most caudal row of the largest component with a width of five voxels or more, and in seven rows above that row. No manual interaction was required. 


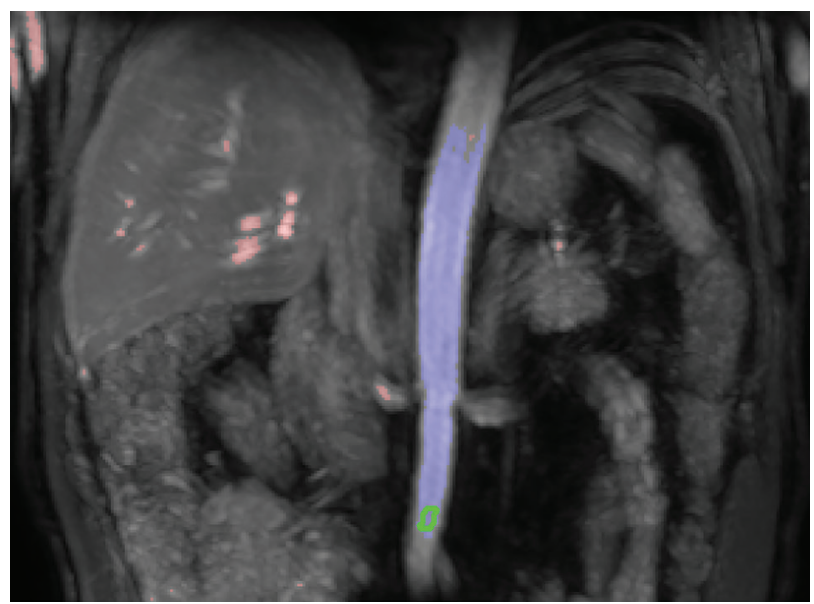

Figure 2. Time MIP of unregistered images used for automated segmentation of aorta ROI. The 2 percent voxels with highest signal intensity are highlighted red and blue. The blue voxels denote the largest connected component, i.e. the aorta. As caudal as possible in this component a $5 \times 8$ voxel ROI is delineated in green. Although not clearly visible in this time MIP, an inflow artefact is present in the cranial parts of the aorta also in this subject.

\section{Registration}

Rigid registration was performed in $3 \mathrm{D}$ to the fat-only first dynamic image using the visualisation toolkit Registration Toolkit (VTK, v2.o.o ${ }^{(27)}$, freely available at http://www.vtk.org/ (Kitware Inc, New York, New York, USA)). To enable separate registration for left and right kidneys, the initial, rough renal masks were employed to create wide crops around both kidneys. Because there is no contrast enhancement in the fat-images used for registration, the choice of the reference image is not pivotal. Prescans were also registered to the first dynamic. The registration procedure is illustrated in Figure 3. In our proposed method, registration is performed on fatimages which are not expected to show contrast enhancement. The obtained transformation matrices are subsequently applied to the corresponding water-images which have identical time-stamps. To compare our method with the conventional approach, we also performed registration on OP-images, which do show contrast enhancement. Of the available images, OP-images have the lowest echo time and are probably most similar to images acquired in a standard postcontrast dynamic series. It would have been more appropriate to perform this comparison with source first echo images, but unfortunately only the reconstructed Dixon images were saved and the source images were not available.

Both for registration to fat and to OP-images gradient cross-correlation (GCC) was used as a similarity measure, but in OP-images normalized mutual information (NMI) was also used to compare performance. Mutual information (MI) in image registration is discussed in detail elsewhere ${ }^{(28)}$. In short, NMI does not depend 
on the actual intensity of the images and therefore might yield better results in registration to contrast enhanced OP-images. Both GCC and NMI are available in the VTK registration toolbox.
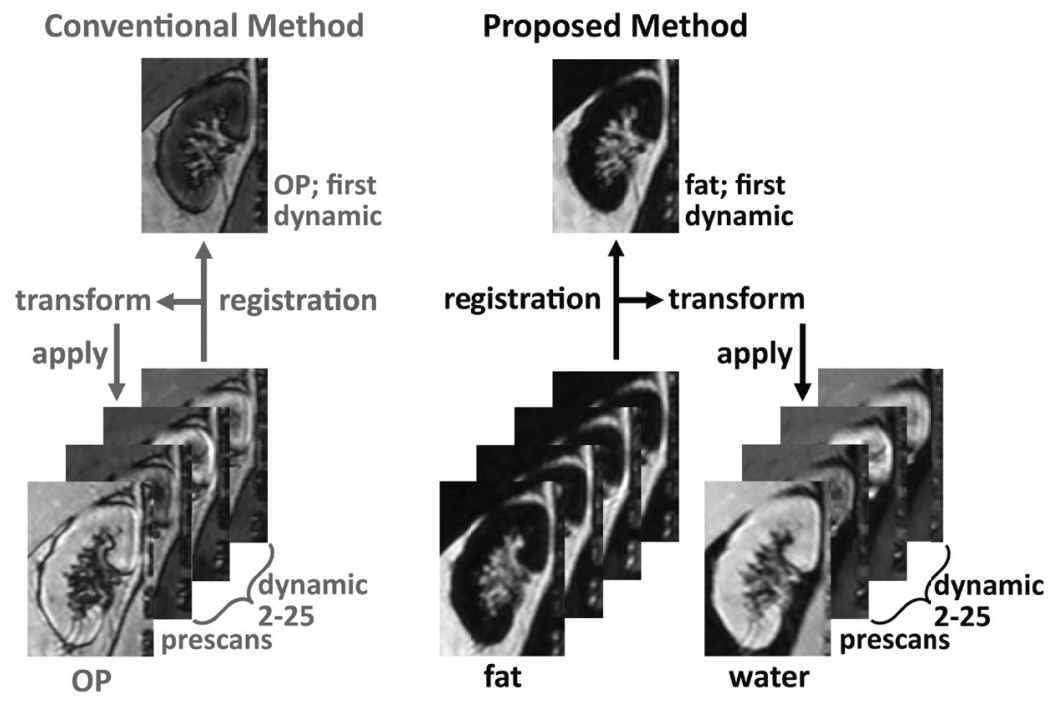

Figure 3. Image registration algorithm. Left conventional method. OP-images were used since they have the shortest echo time of the images available. Registration of the prescans and dynamic 2-25 was performed to the first dynamic and transformation matrices were applied to the OP-images. Right: proposed method. Registration of the prescans and dynamic 2-25 to the first dynamic was performed on fat-images, on which no contrast enhancement is visible. Subsequently, fat transformation matrices were applied to water-images.

transform: transformation matrix

For the arterial ROI in the aorta, the prescans were registered to the first dynamic, because replanning sometimes occurred between the prescans and the dynamics.

To quantify the resulting registration error, two metrics were used. First, as a measure of respiratory induced motion, root mean square (RMS) vertical misalignment of the top of the kidney was measured manually with respect to the first dynamic on all dynamics. This was repeated after registration both to OP and fat-images to measure residual vertical misalignment and to allow for comparison. However, this only measured registration performance in one direction. As a second measure of registration error, the whole parenchyma time-intensity curve was calculated on all dynamics using fat-images. Assuming perfect registration, the timeintensity curve of the whole kidney will be constant. However, because the kidneys are surrounded by adipose tissue, registration errors result in fluctuations in the 
whole kidney time-intensity curve when adipose tissue is shifted inside the renal mask. To quantify registration accuracy, the normalized RMS (NRMS) error of the curve was calculated with respect to the first dynamic:

$$
N R M S=\frac{1}{s_{\max }-s_{\min }} \sqrt{\frac{1}{24} \sum_{i=2}^{25}\left(S_{i}-S_{1}\right)^{2}}
$$

Here, $\mathrm{S}_{\mathrm{i}}$ denotes the value of the whole-parenchyma time-intensity curve at instance $\mathrm{i}$, and $\mathrm{s}_{\max }$ and $\mathrm{s}_{\text {min }}$ denote the maximal and minimal signal intensity in the kidney on the first dynamic. To be able to calculate time-intensity curves on the fat-images, transformation matrices obtained from registration to both fat and OP-images also were applied to the fat-images.

\section{Conversion of Signal to CA Concentration}

For a spoiled gradient echo experiment, the relation between $\mathrm{R}_{1}$ and signal magnitude is given by:

$$
S\left(\alpha, R_{1}\right)=k \rho \frac{\sin (\alpha)\left(1-\exp \left(-T R R_{1}\right)\right)}{1-\cos (\alpha) \exp \left(-T R R_{1}\right)} \exp \left(-T E R_{2}^{*}\right)
$$

Here $\alpha$ denotes the flip angle, $R_{1}$ the spin-lattice relaxation rate, $T R$ the repetition time, TE the echo time and $k \rho$ is a scaling factor to account for proton density and system gain. The influence of $\mathrm{R}_{2}{ }^{*}$ was ignored, which will result in a slight underestimation of $R_{1}$. In the fast exchange limit, $C A$ concentration $C$ is given by:

$$
C \approx \frac{1}{r_{1}}\left(R_{1}-R_{1,0}\right)
$$

Here $R_{1,0}$ denotes precontrast $R_{1}$ and $r_{1}$ is the relaxivity of the $C A$. To estimate contrast agent concentration, joint estimation of pre and postcontrast $\mathrm{R}_{1}$ using a direct fit of the AIF signal to equation 1 was used. The fit was solved using varpro.m ${ }^{(29)}$, a MatLab implementation of the variable projection algorithm ${ }^{(30)}$. In this algorithm, separate solution of the linear and nonlinear parameters reduces covariance between these parameters. In comparable applications, joint estimation has been shown to provide

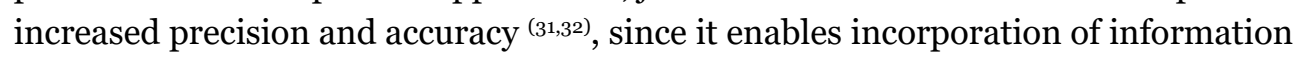
of all dynamics in the estimation of the relaxation times. A thorough analysis of the performance of joint estimation in comparison with other methods to estimate $\mathrm{R}_{1}$ in a DCE experiment is beyond the scope of this paper.

For each voxel, the time-intensity curve over the first three prescans and the 25 dynamics was extracted. Using these $i=28$ measurements (three prescans with flip 
angle 5,13 and $20^{\circ}$ and 25 postscans with flip angle $15^{\circ}$ ), 26 parameters were fitted: the linear parameter $k \rho$ and $n=25$ nonlinear parameters $R_{1, n}$ with $n$ the number of postscans. The function evaluated by varpro.m was defined piecewise:

$$
\frac{S_{i}}{k \rho}=\left\{\begin{array}{l}
\frac{\sin \left(\alpha_{i}\right)\left(1-\exp \left(-T R R_{1,1}\right)\right)}{1-\cos \left(\alpha_{i}\right) \exp \left(-T R R_{1,1}\right)}, \text { if } i \leq 4 \\
\frac{\sin \left(\alpha_{i}\right)\left(1-\exp \left(-T R R_{1, i-3}\right)\right)}{1-\cos \left(\alpha_{i}\right) \exp \left(-T R R_{1, i-3}\right)}, \text { if } i \geq 5
\end{array}\right.
$$

For the three prescans and the first dynamic, that is, the first four measurements, the same value of $R_{1}$ was used. This is reasonable because these scans were performed before CA administration. This forces the algorithm to use the first four measurements to obtain a reasonable estimate of precontrast $\mathrm{R}_{1}$. The combination of prescans and postscans in a single fit enables the calculation of pre and postcontrast $R_{1}$ with a single value for $\mathrm{k} \rho$. The approach was used both for the AIF and CA concentration in the kidneys.

\section{Pharmacokinetic Modeling}

In renal pharmacokinetic modeling, the renal specific two-compartment models of Sourbron ${ }^{(33)}$ or Tofts ${ }^{(34)}$ often are used. In principle, the models are identical, although Sourbron also models tubular outflow. In the supplementary materials, numerical simulations comparing both approaches are described (Figure S1-3). Although the Sourbron model is physiologically more accurate, it yields an extra time constant, $\mathrm{T}_{\mathrm{t}}$, the tubular transit time. For limited temporal resolution and measurement duration, $\mathrm{T}_{t}$ becomes unstable and has a large covariance with the other parameters, especially $\mathrm{K}^{\text {trans }}$ (Figure $\mathrm{S}_{3}$ ). This leads to a markedly increased variance in $\mathrm{F}_{\mathrm{t}}$, resulting in unstable GFR estimates, as shown in the supplementary material (Figure S2 and S3). Therefore the Tofts model was chosen, although it gives a systematic underestimation of GFR (Figure S2). In the Tofts model, two free parameters are fitted: the blood volume $\mathrm{v}_{\mathrm{b}}$ and $\mathrm{K}^{\text {trans }}$, a transfer constant from the intravascular to the extravascular compartment. In the kidney, $\mathrm{K}^{\text {trans }}$ equals the glomerular filtration rate (GFR) per unit volume of tissue. For vascular impulse response function (VIRF), we used a delayed exponential, also yielding two free parameters: $T_{p}$, the time constant in the VIRF and a delay . Together, $T p+$ delay equal the mean residence time. This is dominated by transit time over the renal vascular bed because transit time along the renal artery is very short (about $0.15 \mathrm{~s}$ ) ${ }^{(34)}$. Flow then can be estimated by dividing vb by the mean residence time $\left(\mathrm{T}_{\mathrm{p}}+\right.$ delay). To correct for hematocrit differences between large and small vessels, we used two hematocrit values: 0.41 for large vessels and 0.24 for small vessels. Fitting to the Tofts renal specific model was performed in Matlab for parenchymal ROIs using the trust-region-reflective algorithm. Parenchymal ROIs were used because calculation 
of GFR in a cortical ROI has been shown to underestimate GFR ${ }^{\left({ }^{34}\right)}$. This is reasonable because the model does not account for tubular outflow. In a cortical ROI, there is an outflow of contrast agent to the medulla. Initial values and bounds were partly copied from Sourbron et al. (33): fractional plasma volume vp 0.15 (bounds 0-1); $\mathrm{T}_{\mathrm{p}} 4.5 \mathrm{~s}$ (bounds 1-10), and $\mathrm{K}^{\text {trans }} 40 \mathrm{~mL} / 100 \mathrm{~mL} / \mathrm{min}$ (bounds 6-120). Delay was excluded from the fit and was varied stepwise from 0 to $4 \mathrm{~s}$ in steps of $0.25 \mathrm{~s}$. The Tofts model was fitted both to time-intensity curves obtained from unregistered and fat- and OP-registered images. To generate time-intensity curves, masks generated on fat-registered images were used for consistency.

\section{Statistical Analysis}

The Wilcoxon signed rank test was used to test the difference in registration error between registration to fat-images and OP-images because it does not assume a normal distribution of the data. Spearman's correlation coefficient was used to test correlation between MR based and creatinine based GFR. Here, MR based GFR was corrected for body surface area using the du Bois formula ${ }^{(35)}$. The intraclass correlation coefficient (ICC) was used to test agreement between CKD-EPI based eGFR and DCE based GFR. A p-value of less than 0.05 was considered statistically significant. Analysis was performed with the SPSS 20 (IBM Corp., Armonk, New York,, USA). Values are reported as mean (standard deviation) or median (interquartile range), when appropriate.

\section{Results}

\section{Segmentation}

Segmentation of rough renal masks and aorta ROIs was successful in all subjects. In one subject (patient (P)5), no precise renal mask could be constructed after registration due to heavy respiratory motion, as discussed in detail below. For the remaining subjects, segmentation of precise renal masks was successful.

\section{Registration}

The proposed registration algorithm relies on the absence of contrast enhancement in the Dixon fat-images. Figures $4 a$ and $b$ indeed show near the absence of contrast enhancement in the fat-images, which is contrary to OP-images for which contrast enhancement is clearly visible.

Registration to OP-images was performed using both GCC and NMI as similarity measure. GCC-based registration performed significantly better, resulting in a mean NRMS error of the fat time-intensity curve of 0.08 versus 0.10 for NMIbased registration $(\mathrm{p}=0.005)$. Therefore, $\mathrm{GCC}$ was used as similarity measure in the comparison with registration to fat-images. 

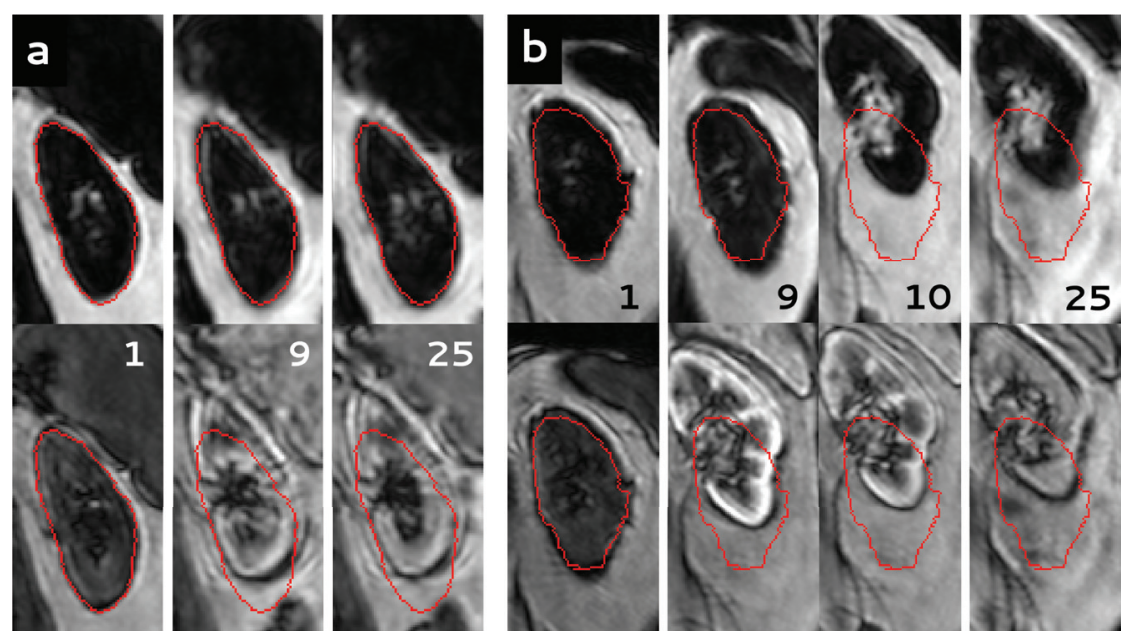

Figure 4. dynamic series of two subjects, registered both to fat-images (upper row) and OP-images (lower row). For clarity, here the fat and OP-images are shown in the upper and lower row, respectively. In red, the contour of the kidney is shown as segmented on the first dynamic. The number of the dynamic is indicated on each image; a) subject $\mathrm{P}_{4}$ : registration to fat-images performs clearly better than registration to OP-images; b) subject $\mathrm{P}_{5}$ : poor registration due to severe respiratory motion during the dynamic series with both techniques.

In Figure 4a, results of registration to both fat and to OP-images in one of the study subjects $\left(\mathrm{P}_{4}\right)$ are shown. Registration to fat-images resulted in good alignment of the kidney, whereas registration to OP-images failed to register the kidney properly, already before CA inflow. In subject $\mathrm{P}_{5}$ (Figure $4 \mathrm{~b}$ ) registration was poor, which was explained by the severe respiratory motion during the dynamic series. This also was illustrated by a RMS vertical misalignment of $58 \mathrm{~mm}$ (left kidney) and $66 \mathrm{~mm}$ (right kidney) before registration. Overall, image quality was acceptable, although later phase images were particularly affected by motion artefacts. In Figure 5 , fat timeintensity curves are shown for four different kidneys. The curves in Figures $5 \mathrm{a}$ and b correspond to the kidneys shown in Figures 4a and b. Difference in registration quality in subject $\mathrm{P}_{4}$ and poor registration in subject $\mathrm{P}_{5}$ are clearly visualized.

In Figure 6, box-plots of the registration errors as measured by RMS vertical misalignment and the fat time-intensity curve are shown (for errors per subject, see Table S1 in the supplementary materials). Respiratory induced RMS vertical misalignment was calculated prior to registration as a measure for initial motion. Residual RMS vertical misalignment was calculated both after registration to fat and OP-images to allow for comparison. NRMS of the fat time-intensity curve is given for all subjects both after registration to fat and OP-images. Respiratory induced motion as measured by RMS vertical misalignment prior to registration was $14 \mathrm{~mm}$ (interquartile range 13) (median over left and right kidneys over all subjects). RMS vertical misalignment was improved more by registration to fat compared to 

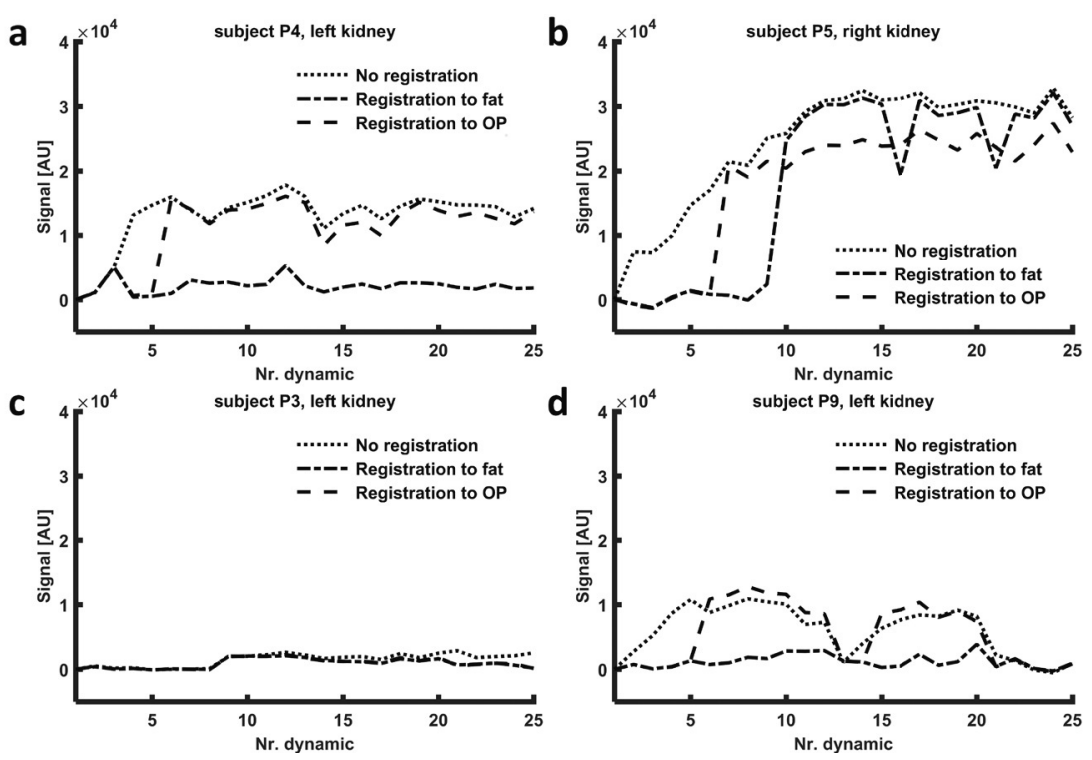

Figure 5. Fat time-intensity curves for four kidneys, calculated on Dixon fat-images both for registration to fat-images and registration to OP-images to compare performance (also the time-intensity curve before registration is shown); a the dynamic series of this kidney is shown in Figure 4a. Registration to fat-images performs evidently better; $\mathbf{b}$ the dynamic series of this kidney is shown in Figure $4 \mathrm{~b}$. Here, both registration to fat and OP-images fails to achieve adequate alignment of the kidneys; c performance of both methods is equal; d although difference is less pronounced than in (a), registration to fat performs clearly better.
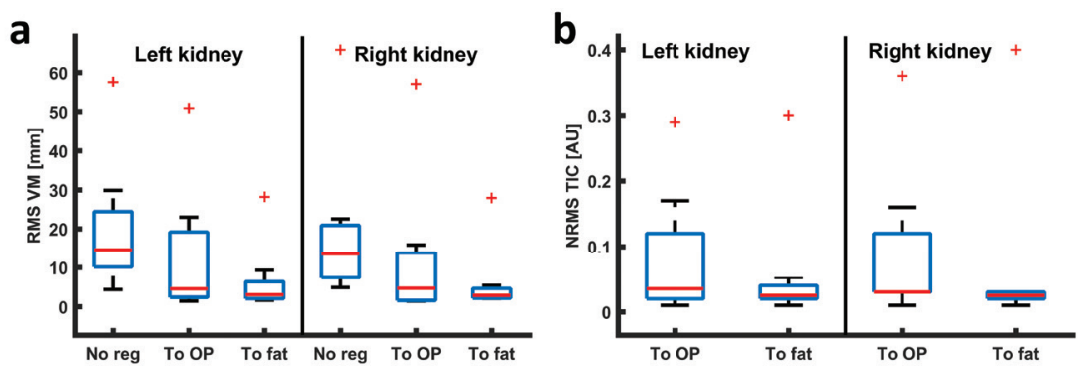

Figure 6. Boxplots of the registration error; a RMS vertical misalignment before registration and after registration to OP and fat-images; $\mathbf{b}$ NRMS error of the time-intensity curve for registration to $\mathrm{OP}$ and registration to fat. In both graphs, the outlier corresponds to subject $\mathrm{P}_{5}$, in whom registration was poor.

registration to OP (median $3^{(3)} \mathrm{mm}$ vs $5^{(13)} \mathrm{mm}$, respectively, $\mathrm{p}=0.03$ ). NRMS of the fat time-intensity curve was smaller for registration to fat, median 0.025 (0.010) compared to 0.030 (0.095) for registration to OP-images (median over left and right kidneys over all subjects, $\mathrm{p}=0.04$ ). Subject $\mathrm{P}_{5}$, for whom registration was poor, was excluded from further analysis. 


\section{Pharmacokinetic Model Fit}

Precontrast arterial T1 $\neg$ as obtained by joint estimation was 1621 (761) ms. Estimated peak arterial contrast agent concentration was 1.o (o.3) mM. Pharmacokinetic model fits were performed on time-intensity curves from unregistered, fat and OP registered images. In Figures $7 \mathrm{a}$ and b, the best and worst obtained pharmacokinetic fits are shown for registration to fat, although even the worst fit is reasonably accurate. The corresponding curves for registration to OP are shown in Figures $7 \mathrm{c}$ and d, where the curve in Figure $7 \mathrm{c}$ is heavily affected by motion due to suboptimal registration. Perfusion and GFR values are provided in Table 1 for all subjects except subject $\mathrm{P}_{5}$. Mean perfusion and GFR were 233 (standard deviation 64) $\mathrm{mL} / 100 \mathrm{~mL} / \mathrm{min}$ and

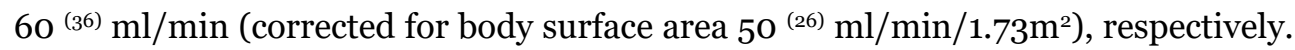
Mean renal volume and fractional blood volume were $172^{(48)} \mathrm{mL}$ and 0.24 (0.10), respectively. Measured renal volume is likely an underestimation because the collecting system and partial volume artefacts were discarded during segmentation. Mean plasma transit time was $4.0(1.4) \mathrm{s}$, with a delay of 1.9 (0.88) s. For registration to OP, mean perfusion and GFR were 265 (174) $\mathrm{mL} / 100 \mathrm{~mL} / \mathrm{min}$ and $84(77) \mathrm{ml} /$ min (corrected for body surface area $68(57) \mathrm{ml} / \mathrm{min} / 1.73 \mathrm{~m}^{2}$ ). Without registration, mean perfusion and GFR were $303(190) \mathrm{ml} / \mathrm{min}$ and $83(83) \mathrm{ml} / \mathrm{min}$ (corrected for body surface area $67(62) \mathrm{ml} / 1.73 \mathrm{~m}^{2} / \mathrm{min}$ ). For registration to OP and unregistered images, pharmacokinetic analysis yielded unphysiological values mainly in subject P9, with perfusion exceeding $500 \mathrm{~mL} / 100 \mathrm{~mL} / \mathrm{min}$ and skGFR exceeding 100/mL/ $\min$.
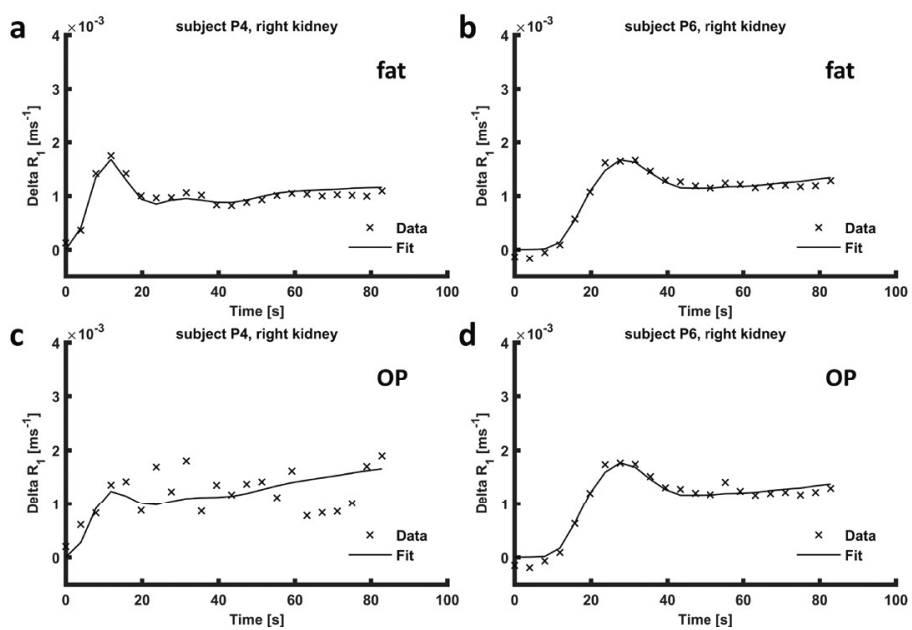

Figure 7. Pharmacokinetic model fits to parenchymal time-concentration curves; a subject P4, in whom the fit to the pharmacokinetic model was worst (registration to fat); $\mathbf{b}$ of subject P6, in whom the best fit was obtained (registration to fat); c time-concentration curve of subject $\mathrm{P} 4$, heavily affected by motion, obtained from OP-registered images; $\mathbf{d}$ time-concentration curve obtained from images registered to OP in subject P6, where registration to OP and to fat performed comparable. 

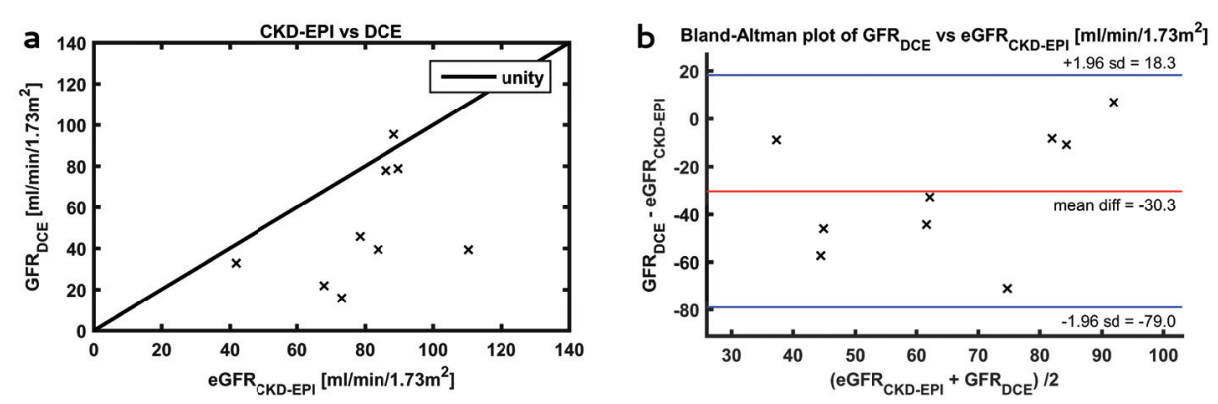

Figure 8. a DCE based GFR compared to eGFR estimated using the CDK-EPI formula; b Bland-Altman plot of the difference between the DCE based GFR and the eGFR.

Creatinine based eGFR is also shown in table 1. In Figure 8, DCE based GFR is plotted against eGFR and a Bland-Altman plot of the difference is shown for registration to fat-images. The correlation coefficient was $0.68(\mathrm{p}=0.04)$, with limited agreement between the measurements as illustrated by an ICC of 0.38. For registration to OP-images, the correlation coefficient was 0.55 without reaching significance $(\mathrm{p}=0.13)$ and the ICC was 0.10 . Without registration, the correlation coefficient was $0.58(\mathrm{p}=0.10)$ and the ICC again was 0.10 .

\section{Discussion}

We described an approach for automated postprocessing in renal DCE MRI, which employs a new approach to image registration. Registration to Dixon fat-images resulted in better registration compared to the conventional method. Furthermore, it improved the estimation of kidney perfusion and GFR, both compared to pharmacokinetic analysis without registration and analysis with registration to OP-images. The proposed registration algorithm relies on the absence of contrast enhancement in fat-images because gadolinium based CAs, which are confined to the extracellular space, do not influence the relaxation of fat-protons, which are contained inside adipocytes. As expected, fat-images hardly showed any contrast enhancement. Registration performed evidently better on fat-images compared to OP-images. However, in one subject with pronounced initial respiratory motion, registration was poor for both approaches. Presumably, this was caused by blurring of the edges of the kidney due to motion artefacts in the fat-images. Contrary to our expectation, GCC based registration performed better than NMI based registration in OP-images, despite visible contrast enhancement. This might be the result of the phase cancellation artefact around the kidneys, present on all dynamics regardless of the timing after contrast inflow.

Because no agreed upon measure for registration quality exists, it is difficult to make a quantitative comparison between registration algorithms. Only Merrem et al. ${ }^{\left({ }^{36)}\right.}$ report coronal motion: the standard deviation of the vertical position of the 
kidney. They report an average initial coronal motion of only $3.4 \mathrm{~mm}$, reducing to $1.7 \mathrm{~mm}$ after deformable registration, a reduction of $50 \%$. In comparison, when we calculate this measure in our dataset, registration to fat-images results in a reduction of 60\%; therefore, performance of Merrem's approach seems comparable to ours. Because Merrem et al. used cross correlation as a similarity measure, which is sensitive to contrast changes during CA inflow, registration could likely be improved further by registration to Dixon fat-images.

Others ${ }^{(19,20,37)}$ proposed registration algorithms based on MI. However, they all used a preregistration module before MI-based registration. Consequently, our method will be easier to implement. Other groups used approaches based on edge detection ${ }^{(8,10,15-18)}$ yielding good results without requiring preregistration modules. However, implementation of the algorithms might be challenging in clinical practice, whereas the registration algorithm we used is freely available. In addition, postprocessing is automated in a graphical user interface and therefore does not require specialist knowledge. Only for segmentation is, manual interaction required to adjust the fat-threshold (optionally), set the number of clusters and label the resultant masks.

In the aorta, calculation of CA concentration could be improved by implementation of the approach recently proposed by Simonis et al. ${ }^{(38)}$. Here, the complex signal is used, instead of magnitude alone. In large vessels parallel to the direction of the static $\left(\mathrm{B}_{\mathrm{o}}\right)$ field, signal phase proves to be linearly related to $\mathrm{CA}$ concentration. In addition, this approach can correct for spatial inhomogeneity of the radiofrequency $\left(B_{1}\right)$ field, which we could not correct for. This is less relevant on 1.5 $\mathrm{T}$ because $\mathrm{B}_{1}$ inhomogeneity is limited, but must be accounted for when moving to higher field strengths. In this analysis, this approach could not be implemented because the source images were not available.

Fitting of the Tofts renal two-compartment model yielded a mean cortical perfusion of $233 \mathrm{~mL} / 100 \mathrm{~mL} / \mathrm{min}$ for fat-registered images. The values obtained using fat-registered images are in good agreement with other renal DCE MRI studies, whereas unregistered and OP-registered images yielded unphysiological values in some kidneys. Sourbron et al. ${ }^{(33)}$ report a perfusion of $229 \mathrm{~mL} / 100 \mathrm{~mL} /$ min, calculated with an instantaneous exponential VIRF. Tofts et al. ${ }^{(34)}$ report a renal flow of $465 \mathrm{~mL} / 100 \mathrm{~mL} / \mathrm{min}$, calculated with a delayed exponential VIRF. The hypertensive population in our study probably explains the relative low perfusion because renal vascular resistance is known to be increased in hypertensive subjects ${ }^{(39)}$. Correlation between eGFR based on creatinine clearance and GFR measured using DCE MRI was significant when fat-registered images were used, although agreement was limited (ICC of 0.38). This is worse compared to results reported recently by Eikefjord et al. ${ }^{(40)}$, who found an ICC of 0.49 in living kidney donors. In nephrectomized subjects, Tipirneni-Sajja et al. found comparable results for 
DCE MR based GFR measurement. However, both authors compared MR based GFR to reference standard methods of GFR measurement: iohexol and ${ }^{99 m} \mathrm{TC}-$ DTPA clearance, respectively. Very good agreement between DCE based GFR and creatinine based GFR was reached by Pandey et al. ${ }^{(41)}$. They used a golden angle stackof-stars approach for data acquisition. From eight different image reconstruction schemes, one resulted in $<5 \%$ discrepancy between the DCE based and creatinine based approach. However, creatinine based GFR provides only a crude estimate of actual GFR. For example, the CKD-EPI formula yields an eGFR within 10\% of the value measured using the reference standard (inulin, iohexol or iothalamate clearance) only for less than $45 \%$ of the subjects ${ }^{(42)}$. According to the same study, it overestimates the GFR in healthy adults on average by more than $10 \%$. In contrast, the Tofts model gives an underestimation of the GFR, which was expected to be about $20 \mathrm{~mL} / 100 \mathrm{~mL} / \mathrm{min}$ according to the simulations in the supplementary materials. This presumably explains the large mean difference between creatinine based eGFR and MR based GFR in our study, which is clearly visualized in the Bland-Altman plot in Figure 8b. Furthermore, the time delay between measurement of the creatinine level and the MRI might be a possible explanation for the limited agreement between creatinine based eGFR and MR based GFR. Biological variation of creatinine clearance is considerable, with a standard deviation of about $12 \%$ for repeated measurements ${ }^{(43)}$. Nevertheless, our DCE protocol can be improved. Most importantly, both the temporal resolution and the duration of measurement have to be increased. The limited temporal resolution and measurement duration prevents us from using the Sourbron model ${ }^{(33)}$. This model is physiologically more accurate because it also models tubular outflow. The simulations in the supplementary materials show this model indeed yields more accurate results compared to the Tofts model. Furthermore, separate analysis for the cortex and medulla can easily be implemented because separate masks already are generated using the k-means clustering approach. Because filtration only occurs in the glomeruli in the cortex, this will likely result in improved accuracy of the GFR measurement. In addition, it enables voxelwise analysis to construct perfusion and filtration maps of the kidney, possibly demonstrating focal differences in renal perfusion and function.

\section{Limitations}

A drawback of our method is the increase in DCE acquisition time inherent to the acquisition of dual-echo images. Despite this we still managed to achieve a temporal resolution of less than 4 seconds, as recommended by Michaely et al. ${ }^{(12)}$. The relatively long acquisition time for $3 \mathrm{D}$ acquisition results in more pronounced respiratory motion artefacts in the images; however, on the fat-images, motion artefacts seem to disturb the contour of the kidney less when compared to OP-images, presumably 
due to the great contrast between the kidney and the surrounding adipose tissue. The temporal resolution can easily be decreased by moving to $3 \mathrm{~T}$ because the twofold increase in the Larmor frequency and hence in the water-fat shift allows in principle allows for a twofold decrease in echo time. In addition, parallel imaging performance is better at $3 \mathrm{~T}$.

Only rigid registration was performed because craniocaudal translation is the dominant motion during respiration in the kidneys. Furthermore, the added value of nonrigid registration is not clear, ${ }^{(15,44)}$ wheras it does result in increased computational time.

Unfortunately, hematocrit values were not available for most subjects; therefore, we had to assume a fixed value for large vessel hematocrit. Likely, perfusion estimates can be improved when incorporating individual hematocrit values ${ }^{(34)}$.

\section{Conclusion}

We have demonstrated the feasibility of automated postprocessing in renal DCE MRI. To handle respiratory motion, one of the main challenges in renal DCE MRI, image registration to fat-images was employed. Registration quality was superior to registration to OP-images thanks to negligible contrast enhancement in fat-images. The method was robust, being able to register nine out of ten images in a satisfactory manner. Because manual interaction was limited, processing does not require specialist knowledge. Therefore, it will be easy to implement in clinical practice. A drawback of this method is the cost in imaging time, resulting in a temporal resolution of $3.9 \mathrm{~s}$; however, straightforward reduction in dynamic imaging time can be achieved by migrating to $3 \mathrm{~T}$. After implementation of the improvements mentioned in the discussion, the next step will be to validate the GFR obtained using this renal DCE-MRI protocol in healthy volunteers compared to a reference standard such as inulin clearance.

\section{Acknowledgements}

AdB was supported by an Alexandre Suerman scholarship for MD/PhD students of the University Medical Center Utrecht, the Netherlands. The authors would like to thank Allessandro Sbrizzi for his support in the implementation of the VARPRO algorithm. 


\section{References}

1. Zöllner FG, Zimmer F, Klotz S, Hoeger S, Schad LR. Renal perfusion in acute kidney injury with DCE-MRI: deconvolution analysis versus two-compartment filtration model. Magnetic resonance imaging. 2014;32(6):781-5.

2. Buckley DL, Shurrab AE, Cheung CM, Jones AP, Mamtora H, Kalra PA. Measurement of single kidney function using dynamic contrast-enhanced MRI: comparison of two models in human subjects. J Magn Reson Imaging. 2006;24(5):1117-23.

3. Chapman SJ, Wah TM, Sourbron SP, Buckley DL. The effects of cryoablation on renal cell carcinoma perfusion and glomerular filtration rate measured using dynamic contrast-enhanced MRI: a feasibility study. Clinical radiology. 2013;68(9):887-94.

4. Eikefjord E, Andersen E, Hodneland E, Zollner F, Lundervold A, Svarstad E, et al. Use of $3 \mathrm{D}$ DCE-MRI for the estimation of renal perfusion and glomerular filtration rate: an intrasubject comparison of FLASH and KWIC with a comprehensive framework for evaluation. AJR Am J Roentgenol. 2015;204(3):W273-81.

5. Notohamiprodjo M, Sourbron S, Staehler M, Michaely HJ, Attenberger UI, Schmidt GP, et al. Measuring perfusion and permeability in renal cell carcinoma with dynamic contrast-enhanced MRI: a pilot study. J Magn Reson Imaging. 2010;31(2):490-501.

6. Notohamiprodjo M, Staehler M, Steiner N, Schwab F, Sourbron SP, Michaely HJ, et al. Combined diffusion-weighted, blood oxygen level-dependent, and dynamic contrastenhanced MRI for characterization and differentiation of renal cell carcinoma. Acad Radiol. 2013;20(6):685-93.

7. Wang W, Ding J, Li Y, Wang C, Zhou L, Zhu H, et al. Magnetic resonance imaging and computed tomography characteristics of renal cell carcinoma associated with Xp11.2 translocation/TFE3 gene fusion. PLoS One. 2014;9(6):e99990.

8. Khalifa F, El-Baz A, Gimel'farb G, Abu El-Ghar M. Non-invasive image-based approach for early detection of acute renal rejection. Med Image Comput Comput Assist Interv. 2010;13(Pt 1):10-8.

9. Khalifa F, Abou El-Ghar M, Abdollahi B, Frieboes HB, El-Diasty T, El-Baz A. A comprehensive non-invasive framework for automated evaluation of acute renal transplant rejection using DCE-MRI. NMR Biomed. 2013;26(11):1460-70.

10. Liu W, Sung K, Ruan D. Shape-based motion correction in dynamic contrast-enhanced MRI for quantitative assessment of renal function. Med Phys. 2014;41(12):122302.

11. Dujardin M, Sourbron S, Luypaert R, Verbeelen D, Stadnik T. Quantification of renal perfusion and function on a voxel-by-voxel basis: a feasibility study. Magn Reson Med. 2005;54(4):841-9.

12. Michaely HJ, Sourbron SP, Buettner C, Lodemann KP, Reiser MF, Schoenberg SO. Temporal constraints in renal perfusion imaging with a 2-compartment model. Invest Radiol. 2008;43(2):120-8. 
13. Palmowski M, Schifferdecker I, Zwick S, Macher-Goeppinger S, Laue H, Haferkamp A, et al. Tumor perfusion assessed by dynamic contrast-enhanced MRI correlates to the grading of renal cell carcinoma: initial results. Eur J Radiol. 2010;74(3):e176-80.

14. Attenberger UI, Sourbron SP, Michaely HJ, Reiser MF, Schoenberg SO. Retrospective respiratory triggering renal perfusion MRI. Acta Radiol. 2010;51(10):1163-71.

15. Zikic D, Sourbron S, Feng X, Michaely HJ, Khamene A, Navab N, editors. Automatic alignment of renal DCE-MRI image series for improvement of quantitative tracer kinetic studies. Proceedings of SPIE 6914, Medical Imaging: Image Processing; 2008; San Diego, CA.

16. de Senneville BD, Mendichovszky IA, Roujol S, Gordon I, Moonen C, Grenier N. Improvement of MRI-functional measurement with automatic movement correction in native and transplanted kidneys. J Magn Reson Imaging. 2008;28(4):970-8.

17. Song T, Lee VS, Rusinek H, Kaur M, Laine AF. Automatic 4-D registration in dynamic MR renography based on over-complete dyadic wavelet and Fourier transforms. Med Image Comput Comput Assist Interv. 2005;8(Pt 2):205-13.

18. Yang X, Ghafourian P, Sharma P, Salman K, Martin D, Fei B. Nonrigid Registration and Classification of the Kidneys in 3D Dynamic Contrast Enhanced (DCE) MR Images. Proc SPIE Int Soc Opt Eng. 2012;8314:83140B.

19. Zöllner FG, Sance R, Rogelj P, Ledesma-Carbayo MJ, Rørvik J, Santos A, et al. Assessment of $3 \mathrm{D}$ DCE-MRI of the kidneys using non-rigid image registration and segmentation of voxel time courses. Comput Med Imaging Graph. 2009;33(3):171-81.

20. Positano V, Bernardeschi I, Zampa V, Marinelli M, Landini L, Santarelli MF. Automatic 2D registration of renal perfusion image sequences by mutual information and adaptive prediction. Magn Reson Mater Phy. 2013;26(3):325-35.

21. Hodneland E, Hanson EA, Lundervold A, Modersitzki J, Eikefjord E, Munthe-Kaas AZ. Segmentation-driven image registration- application to 4D DCE-MRI recordings of the moving kidneys. IEEE Trans Image Process. 2014;23(5):2392-404.

22. Hodneland E, Lundervold A, Rorvik J, Munthe-Kaas AZ. Normalized gradient fields for nonlinear motion correction of DCE-MRI time series. Comput Med Imaging Graph. 2014;38(3):202-10.

23. Cheng KT. Gadobutrol. 2007 [cited 2017 Mar 14]. In: Molecular Imaging and Contrast Agent Database (MICAD) [Internet]. Bethesda MD: National Center for Biotechnology Information (US), [cited 2017 Mar 14]. Available from: https://www.ncbi.nlm.nih. gov/books/NBK23589/.

24. Vink EE, De Boer A, Hoogduin HJM, Voskuil M, Leiner T, Bots ML, et al. Renal BOLDMRI relates to kidney function and activity of the renin-angiotensin-aldosterone system in hypertensive patients. Journal of Hypertension. 2015;33(3):597-604.

25. Levey AS, Stevens LA, Schmid CH, Zhang YL, Castro AF, 3rd, Feldman HI, et al. A new equation to estimate glomerular filtration rate. Ann Intern Med. 2009;150(9):604-12.

26. Eggers H, Brendel B, Duijndam A, Herigault G. Dual-echo Dixon imaging with flexible 
choice of echo times. Magn Reson Med. 2011;65(1):96-107.

27. Hartkens T, Rueckert D, Schnabel JA, Hawkes DJ, Hill DLG, editors. VTK CISG Registration Toolkit An Open Source Software Package for Affine and Non-rigid Registration of Single- and Multimodal 3D Images. Proceedings of the workshop Bildverarbeitung für die Medizin; 2002 2002/o1/o1; Leipzig (DE): Springer Berlin Heidelberg.

28. Pluim JPW, Maintz JBA, Viergever MA. Mutual-information-based registration of medical images: a survey. IEEE Trans Med Imaging. 2003;22(8):986-1004.

29. O'Leary DP, Rust BW. Variable projection for nonlinear least squares problems. Comput Optim Appl. 2012;54(3):579-93.

30. Golub G, Pereyra V. Separable nonlinear least squares: the variable projection method and its applications. Inverse problems. 2003;19(2):R1.

31. Dickie BR, Banerji A, Kershaw LE, McPartlin A, Choudhury A, West CM, et al. Improved accuracy and precision of tracer kinetic parameters by joint fitting to variable flip angle and dynamic contrast enhanced MRI data. Magn Reson Med. 2016;76(4):1270-81.

32. Teixeira RP, Malik SJ, Hajnal JV. Joint system relaxometry (JSR) and CramerRao lower bound optimization of sequence parameters: A framework for enhanced precision of DESPOT T1 and T2 estimation. Magn Reson Med. 2017.

33. Sourbron SP, Michaely HJ, Reiser MF, Schoenberg SO. MRI-measurement of perfusion and glomerular filtration in the human kidney with a separable compartment model. Invest Radiol. 2008;43(1):40-8.

34. Tofts PS, Cutajar M, Mendichovszky IA, Peters AM, Gordon I. Precise measurement of renal filtration and vascular parameters using a two-compartment model for dynamic contrast-enhanced MRI of the kidney gives realistic normal values. Eur Radiol. 2012;22(6):1320-30.

35. Du Bois D, Du Bois EF. A formula to estimate the approximate surface area if height and weight be known. 1916. Nutrition. 1989;5(5):303-11; discussion 12-3.

36. Merrem AD, Zollner FG, Reich M, Lundervold A, Rorvik J, Schad LR. A variational approach to image registration in dynamic contrast-enhanced MRI of the human kidney. Magn Reson Imaging. 2013;31(5):771-7.

37. Sance R, Ledesma-Carbayo MJ, Lundervold A, Santos A, editors. Alignment of 3D DCE-MRI abdominal series for optimal quantification of kidney function. Proceedings

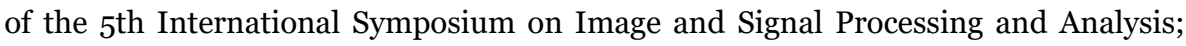
2007; Istanbul (TR): IEEE.

38. Simonis FF, Sbrizzi A, Beld E, Lagendijk JJ, van den Berg CA. Improving the arterial input function in dynamic contrast enhanced MRI by fitting the signal in the complex plane. Magn Reson Med. 2016;76(4):1236-45.

39. Pedersen EB, Kornerup HJ. Renal haemodynamics and plasma renin in patients with essential hypertension. Clin Sci Mol Med. 1976;50(5):409-14.

40. Eikefjord E, Andersen E, Hodneland E, Svarstad E, Lundervold A, Rorvik J. 
Quantification of Single-Kidney Function and Volume in Living Kidney Donors Using Dynamic Contrast-Enhanced MRI. AJR Am J Roentgenol. 2016:1-9.

41. Pandey A, Yoruk U, Keerthivasan M, Galons JP, Sharma P, Johnson K, et al. Multiresolution imaging using golden angle stack-of-stars and compressed sensing for dynamic MR urography. J Magn Reson Imaging. 2017;46(1):303-11.

42. Pottel H, Hoste L, Dubourg L, Ebert N, Schaeffner E, Eriksen BO, et al. An estimated glomerular filtration rate equation for the full age spectrum. Nephrol Dial Transplant. 2016;31(5):798-806.

43. Greenblatt DJ, Ransil BJ, Harmatz JS, Smith TW, Duhme DW, Koch-Weser J. Variability of 24-hour urinary creatinine excretion by normal subjects. J Clin Pharmacol. 1976;16(7):321-8.

44. Rogelj P, Zoellner FG, Kovačič S, Lundervold A, editors. Motion correction of contrast-enhanced MRI time series of kidney. Proceedings of the 16th International Electrotechnical and Computer Science Conference; 2007; Portoroz (SI): Citeseer. 


\section{Supplementary materials}

\section{Pharmacokinetic modelling}

\section{Simulations}

In renal pharmacokinetic modelling, often the renal specific two-compartment models of Sourbron ${ }^{(1)}$ or Tofts ${ }^{(2)}$ are used. In principle, the models are identical, although Sourbron also models tubular outflow. Although physiologically more accurate, this implies fitting of an extra parameter, which increases the risk of overfitting. Simulations were performed to motivate the choice between these models.

To generate ground-truth data, a generic arterial input function (AIF) modelled by Parker et al. ${ }^{(3)}$ was used, which is shown in Figure S1. To model the time-dependent change in contrast agent concentration in kidney tissue, Sourbron's model ${ }^{(1)}$ was used, which was considered most accurate since it also models tubular outflow. Parameters used are: Ht 0.41, vb 0.30, Tp 5.5s, delay 1.0 s, perfusion $\left(F_{b}=\frac{v_{b}}{T_{p}+D}\right) 277 \mathrm{~mL} / 100 \mathrm{~mL} / \mathrm{min}, \mathrm{T}_{\mathrm{t}} 125 \mathrm{~s}, \mathrm{~F}_{\mathrm{t}} 30 \mathrm{~mL} / 100 \mathrm{~mL} / \mathrm{min}$. Both the AIF and the tissue-concentration curve were modelled in high resolution $(100 \mathrm{~Hz})$ and down sampled to the desired temporal resolution (both curves and the sampling points are shown in Figure S1). Subsequently, Gaussian white noise was added to both the AIF and the tissue-concentration curve. For varying measurement durations and sampling frequencies both Tofts' and Sourbron's model were fitted to these curves. For every set of parameters, 300 iterations were performed. Figures S2a and b show the dependence of perfusion and GFR estimation of temporal resolution. For GFR (Figure S2b), precision of Sourbron's model decreases markedly with decreasing temporal resolution. Presumably, this can be explained by overfitting due to the need of fitting an extra parameter (the tubular transit time, $\mathrm{T}_{\mathrm{t}}$ ). As shown in Figure S3a, decreasing measurement time leads to a substantial increase in variance of $T_{t}$. Furthermore, it leads to increased covariance between $T_{t}$ and all other parameters (Figure $\mathrm{S}_{3} b$ ). Normalized covariance of $\mathrm{T}_{t}$ and $\mathrm{F}_{t}$ is largest, resulting in a marked increase in variance of Ft and therefore GFR when measurement time decreases. Precision of Tofts' model for GFR estimation is larger and less dependent of temporal resolution. However, while accurate in perfusion estimation, Tofts' model gives a systematic underestimation of GFR. Accuracy is better for Sourbron's model, especially for the higher temporal resolutions.

For the dependence of the pharmacokinetic analysis on measurement duration similar results were obtained: accurate, but rather imprecise values of GFR for Sourbron's model and precise estimates for Tofts' model, although not accurate for GFR (Figure S2c and d). Tofts' model gives an underestimation of GFR which increases with increasing measurement time (Figure S2d). 


\section{Conclusion}

The results of these simulations support the choice for Tofts' model for the analysis of our data acquired with limited measurement duration (98.5 $\mathrm{s}$ ) and limited temporal resolution (3.94s). With this limited data, Tofts' model gives comparable estimations of perfusion, compared to Sourbron's model. However, we have to accept a rather large, systematic underestimation of GFR. For data acquired with higher temporal resolution and longer measurement duration, the choice for Sourbron's model can be more appropriate.

\section{References}

1. Sourbron SP, Michaely HJ, Reiser MF, Schoenberg SO. MRI-measurement of perfusion and glomerular filtration in the human kidney with a separable compartment model. Invest Radiol 2008;43:40-48.

2. Tofts PS, Cutajar M, Mendichovszky IA, Peters AM, Gordon I. Precise measurement of renal filtration and vascular parameters using a two-compartment model for dynamic contrast-enhanced MRI of the kidney gives realistic normal values. Eur Radiol 2012;22:1320-1330.

3. Parker GJ, Roberts C, Macdonald A, et al. Experimentally-derived functional form for a population-averaged high-temporal-resolution arterial input function for dynamic contrast-enhanced MRI. Magn Reson Med 2006;56:993-1000. 
Table S1. Baseline and follow-up data.

\begin{tabular}{|c|c|c|c|c|c|c|c|c|c|c|}
\hline \multirow{3}{*}{ Registation } & \multicolumn{5}{|c|}{ Left kidney } & \multicolumn{5}{|c|}{ Right kidney } \\
\hline & \multirow{2}{*}{$\begin{array}{c}- \\
\text { VM } \\
{[\mathbf{m m}]}\end{array}$} & \multicolumn{2}{|c|}{ To OP } & \multicolumn{2}{|c|}{ To fat } & \multirow{2}{*}{$\begin{array}{c}- \\
\text { VM } \\
{[\mathbf{m m}]}\end{array}$} & \multicolumn{2}{|c|}{ To OP } & \multicolumn{2}{|c|}{ To fat } \\
\hline & & $\begin{array}{c}\mathrm{VM} \\
{[\mathrm{mm}]}\end{array}$ & $\begin{array}{c}\text { TIC } \\
{[-]}\end{array}$ & $\begin{array}{c}\mathrm{VM} \\
{[\mathrm{mm}]}\end{array}$ & $\begin{array}{c}\text { TIC } \\
{[-]}\end{array}$ & & $\begin{array}{c}\text { VM } \\
{[\mathbf{m m}]}\end{array}$ & $\begin{array}{c}\text { TIC } \\
{[-]}\end{array}$ & $\begin{array}{c}\text { VM } \\
{[\mathbf{m m}]}\end{array}$ & $\begin{array}{l}\text { TIC } \\
{[-]}\end{array}$ \\
\hline $\mathrm{P} 1$ & 7 & 1 & 0.04 & 2 & 0.04 & 6 & 1 & 0.03 & 3 & 0.03 \\
\hline P2 & 10 & 2 & 0.03 & 2 & 0.02 & 18 & 13 & 0.08 & 5 & 0.03 \\
\hline $\mathrm{P}_{3}$ & 4 & 5 & 0.02 & 3 & 0.02 & 7 & 3 & 0.03 & 3 & 0.02 \\
\hline $\mathrm{P}_{4}$ & 30 & 23 & 0.17 & 5 & 0.03 & 23 & 16 & 0.16 & 2 & 0.02 \\
\hline $\mathrm{P}_{5}$ & 58 & 51 & 0.29 & 28 & 0.30 & 66 & 57 & 0.36 & 28 & 0.40 \\
\hline P6 & 16 & 9 & 0.01 & 9 & 0.01 & 5 & 4 & 0.01 & 5 & 0.01 \\
\hline P7 & 13 & 4 & 0.02 & 2 & 0.02 & 11 & 5 & 0.03 & 4 & 0.03 \\
\hline P8 & 10 & 3 & 0.05 & 3 & 0.05 & 12 & 1 & 0.02 & 2 & 0.02 \\
\hline P9 & 24 & 19 & 0.12 & 6 & 0.03 & 21 & 14 & 0.12 & 2 & 0.02 \\
\hline P10 & 17 & 1 & 0.03 & 2 & 0.02 & 15 & 2 & 0.03 & 2 & 0.03 \\
\hline Median & 15 & 5 & 0.035 & 3 & 0.025 & 13 & 5 & o.030 & 3 & 0.025 \\
\hline IQR & 14 & 17 & 0.100 & 4 & 0.020 & 14 & 12 & 0.090 & 2 & 0.010 \\
\hline
\end{tabular}

VM: root mean square vertical misalignment; TIC: normalized root mean square error of time-intensity curve of fat-image; IQR: interquartile range.

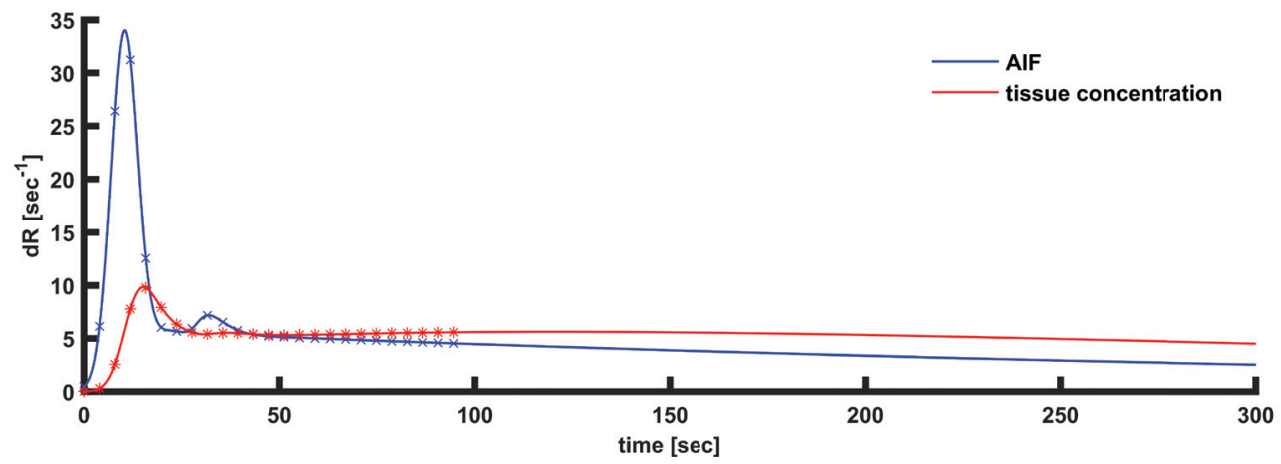

Figure S1. Example of tissue-concentration curve and AIF (generic AIF as modelled by Parker et al. (S3)) used for determination of fit accuracy. In solid lines the high resolution curve is depicted, the sampling points denote the measurements in our MR sequence. Sample points: dt $3.94 \mathrm{~s}$, tmax $98.5 \mathrm{~s}$. 

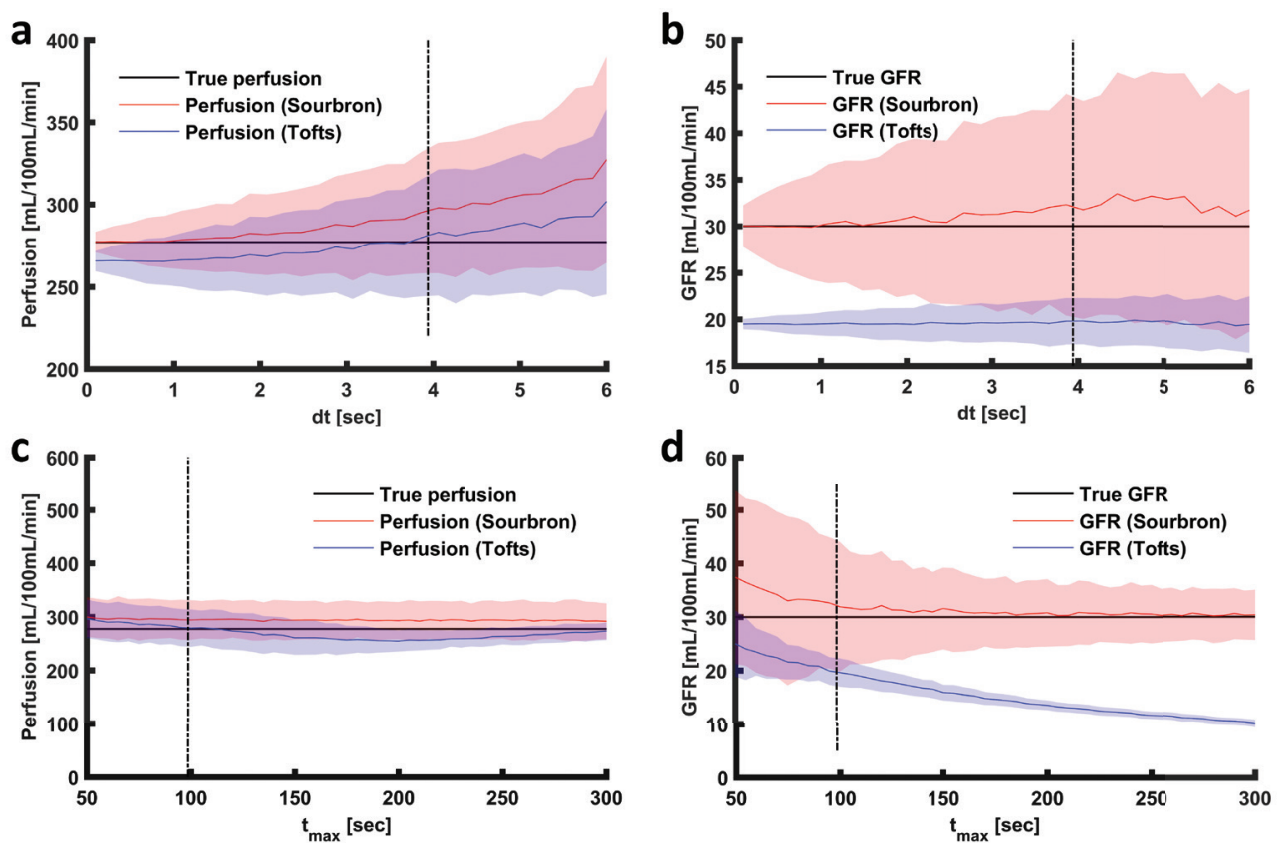

Figure S2. Comparison of perfusion and GFR estimated using Sourbron's and Tofts' models. The shaded area denotes 1.96 times the standard deviation. The black horizontal line denotes true perfusion or GFR and the dotted black vertical lines denote $\mathrm{dt}$ and $\mathrm{t}_{\max }$ of our data (3.94 $\mathrm{s}$ and $98.5 \mathrm{~s}$, respectively); a dependence of perfusion estimation on decreasing temporal resolution (or increasing dt on the x-axis); b GFR dependence on temporal resolution; c estimation of perfusion, dependence on duration of measurement $\left(\mathrm{t}_{\max }\right)$ and $\mathbf{d}$ the same for GFR.

a

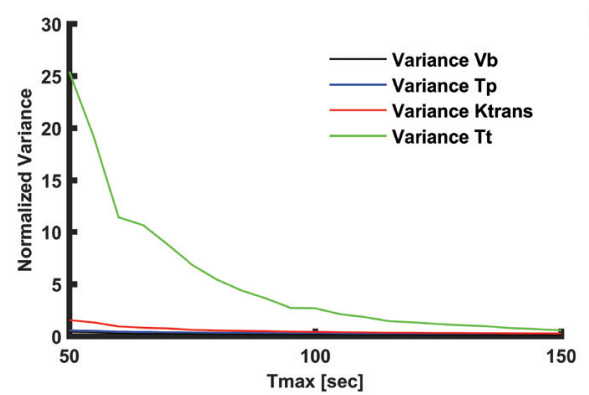

b

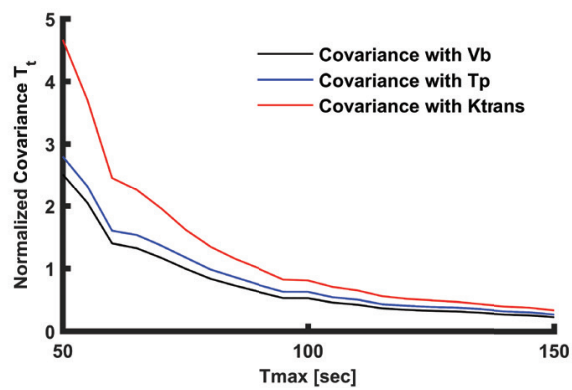

Figure S3. a Normalized variance $\left(\sqrt{\frac{\operatorname{Var}(x)}{\mu_{x}^{2}}}\right)$ of $\mathrm{Tt}$ (tubular transit time) and the other fit parameters versus measurement time. Note that the variance of $\mathrm{T}_{\mathrm{t}}$ exceeds the variance of the other parameters by far; $\mathbf{b}$ normalized covariance $\left(\sqrt{\frac{\operatorname{Var}(x y)}{\mu_{x} \mu_{y}}}\right)$ of $\mathrm{T}_{\mathrm{t}}$ with the other
parameters as a function of measurement time. 


$$
\text { की }
$$




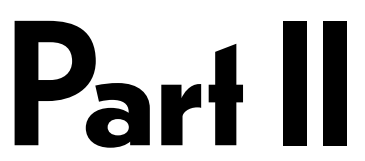

Clinical Applications 



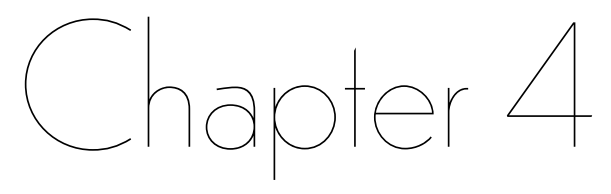

\section{Decreased Native Renal $T_{1}$ up to $O$ ne Week after Gadobutrol Administration in Healthy Volunteers}

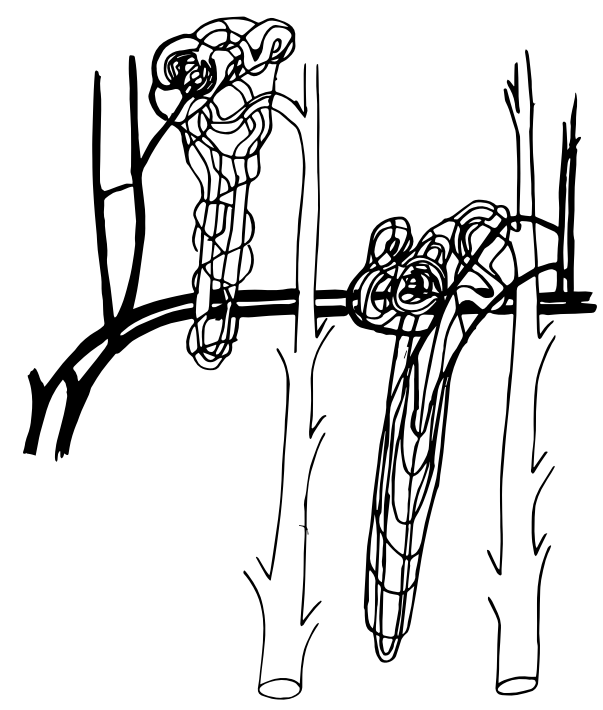

Author contributions

de Boer A, Harteveld AA, Pieters TT, Blankestijn PJ, Bos C, Froeling M, Joles JA, Verhaar MC, Leiner T, Hoogduin JM

Published in

J Magn Reson Imaging 2019; doi:10.1002/jmri.27014 


\section{Abstract}

Background Gadolinium based contrast agents (GBCAs) are widely used in MRI, despite safety concerns regarding deposition in brain and other organs. In animal studies gadolinium was detected for weeks after administration in the kidneys, but this has not yet been demonstrated in humans.

Purpose To find evidence for the prolonged presence of gadobutrol in the kidneys in healthy volunteers.

Study type Combined retrospective and prospective analysis of a repeatability study

Population Twenty-three healthy volunteers with normal renal function (12 women, age range 40-76 years), of whom 21 were used for analysis.

Fieldstrength/Sequence inversion recovery based $\mathrm{T}_{1}$ map at $3 \mathrm{~T}$

Assessment $\mathrm{T}_{1}$ maps were obtained twice with a median interval of 7 (range: 4-16) days. The $\mathrm{T}_{1}$ difference ( $\left.\Delta \mathrm{T} 1\right)$ between both scans was compared between the gadolinium group $\left(\mathrm{N}=16,0.05 \mathrm{mmol} / \mathrm{kg}\right.$ gadobutrol administered after $\mathrm{T}_{1}$ mapping during both scan sessions) and the control group ( $\mathrm{N}=5$, no gadobutrol). $\mathrm{T}_{1}$ maps were analyzed separately for cortex and medulla.

Statistical tests Mann-Whitney-U tests to detect differences in $\Delta \mathrm{T}_{1}$ between groups and linear regression to relate time between scans and estimated glomerular filtration rate (eGFR) to $\Delta \mathrm{T}_{1}$.

Results $\Delta \mathrm{T}_{1}$ differed significantly between gadolinium and control group: median $\Delta \mathrm{T}_{1}$ cortex $-98 \mathrm{vs} 7 \mathrm{~ms}(\mathrm{p}<0.001)$ and medulla $-68 \mathrm{~ms}$ vs $19 \mathrm{~ms}$ ( $\left.\mathrm{p}=0.001\right)$, respectively. The bias corresponds to renal gadobutrol concentrations of $8 \mathrm{nmol} / \mathrm{g}$ tissue (cortex) and $4 \mathrm{nmol} / \mathrm{g}$ tissue (medulla), ie approximately $2.4 \mu \mathrm{mol}$ for both kidneys (0.05\% of original dose). $\Delta \mathrm{T}_{1}$ correlated in the gadolinium group with duration between acquisitions for both cortex (regression coefficient $(\beta) 16.5 \mathrm{~ms} /$ day, $\mathrm{R}^{2} \mathrm{0} .5 \mathrm{O}, \mathrm{p}<0.001$ ) and medulla ( $\beta$ 11.5ms/day, $\mathrm{R}^{2}$ o.32, $\left.\mathrm{p}<0.001\right)$. Medullary $\Delta \mathrm{T}_{1}$ correlated with eGFR $\left(\beta 1.13 \mathrm{~ms} /(\mathrm{ml} / \mathrm{min}) \mathrm{R}^{2} 0.25, \mathrm{p}=0.008\right)$.

Data conclusion We found evidence of delayed renal gadobutrol excretion after a single contrast agent administration in subjects with normal renal function. Even within this healthy population, elimination delay increased with decreasing kidney function. 


\section{Introduction}

Gadolinium based contrast agents (GBCAs) are widely used in magnetic resonance imaging (MRI). However, since an association between GBCAs and nephrogenic systemic fibrosis (NSF) was described in 2005-2006 ${ }^{(1,2)}$, use of GBCAs in subjects with severe renal disease has been avoided. NSF was mainly associated with linear GBCAs, with typically lower kinetic stability compared to macrocyclic GBCAs. ${ }^{(3)}$ Therefore, in Europe linear GBCAs have largely been abandoned in favor of macrocyclic GBCAs. ${ }^{(3)}$ These preventive measures were effective. Since 2008, NSF has been practically eliminated. ${ }^{(4)}$ However, in 2014, a report was published suggesting long-term deposition of GBCAs in the dentate nucleus and globus pallidus $^{(5)}$, which was later confirmed by postmortem investigation of brains from patients (with median estimated glomerular filtration rate (eGFR) $88 \mathrm{~mL} / \mathrm{min} / 1.73$ $\mathrm{m}^{2}$ ) who underwent repeated contrast-enhanced MR exams. Other investigators have reported gadolinium deposition in bone ${ }^{(6)}$ and skin ${ }^{(7)}$ of subjects without renal damage after contrast-enhanced MR exams. Thus far, clinical importance of this deposition is unknown. Gadolinium deposition has never been linked to adverse effects in well-controlled studies. Apart from NSF, for which the link to linear GBCAs is well established, some chronic nonallergic symptoms have been reported in a small number of patients in uncontrolled observational studies ${ }^{(3)}$ Most evidence origins either from retrospective data ${ }^{(8)}$ or from studies focused on immediate adverse reactions with short follow-up. ${ }^{(9)}$ Some large observational studies with longer follow-up (4-20 months) have focused on the development of NSF, but did not include endpoints associated with gadolinium deposition in other organs, like neurotoxicity. ${ }^{(10-12)}$

Most GBCAs are cleared almost exclusively via the kidneys, with the exception of those agents specifically designed for liver imaging, which are partially cleared via the hepatobiliary system. ${ }^{(13)}$ In male, healthy subjects, $98 \%$ of gadobutrol was excreted within 12 hours. ${ }^{(14)}$ For extracellular contrast agents like gadobutrol, the plasma concentration was initially described by a two-phase pharmacokinetic model. A distribution phase over the entire extracellular volume with a sharp decline in contrast agent is followed by a slower, exponential decay in the (renal) clearance phase. ${ }^{(13)}$ Extracellular GBCAs are typically freely filtered, with a clearance rate which equals the GFR. ${ }^{(13)}$ However, this model was not compatible with the above mentioned reports of long-term deposition. Based on animal data and retrospective analysis of urine collections in humans, the existence of a so-called deep compartment was proposed, possibly bone, from which GBCA is slowly released. ${ }^{\left({ }^{15}\right)}$ However, "to date, no clinical study allows to assess the pharmacokinetics of the GBCAs in human bone" according to E. Lancelot ${ }^{(15)}$. As far as the authors are aware, apart from case reports indeed no additional studies have been published on long-term GBCA 
pharmacokinetics in humans, so this model has yet to be confirmed.

Renal multiparametric MRI, including quantitative dynamic contrast enhanced (DCE) MRI, is increasingly being studied as a diagnostic method in renal parenchymal diseases. To validate this method, we recently performed a repeatability study in healthy volunteers using a comprehensive renal MRI protocol. The scan protocol included, among others, $\mathrm{T}_{1}$ mapping of the kidney before contrast media injection (native or precontrast $\mathrm{T}_{1}$ ) and DCE imaging. Unexpectedly, a systematic negative bias in the repeated $\mathrm{T}_{1}$ measurements of the kidney was observed. Since gadobutrol is designed to induce a $\mathrm{T}_{1}$ decrease, this difference might be explained by the presence of trace amounts of remaining contrast agent from gadobutrol (a macrocyclic GBCA) injection during the previous scan session. However, such a finding would be unexpected since the plasma half-life of gadobutrol is reported to be around 1.8 hour. ${ }^{(16)}$ Assuming an extracellular volume of $15 \mathrm{~L}$ and an initial dose of $4 \mathrm{mmol}$, a plasma concentration of around $3^{*} 10^{-23} \mathrm{nmol} / \mathrm{mL}$ would be expected after a week ( 93 half-lives), which is virtually nothing. The presence of trace amounts of gadobutrol after a week therefore would contradict the two-phase pharmacokinetic model and support the hypothesis of higher order kinetics. Therefore, this study aims to find evidence for the presence of gadobutrol in the kidneys up to one week after administration in healthy volunteers.

\section{Methods}

The analyses were performed on two human datasets. The first dataset was acquired as part of the ReMaRK study (Repeatability of functional Magnetic Resonance imaging of the Kidneys) to assess interscan repeatability of a multiparametric renal MRI examination that included contrast agent administration and was used retrospectively in this analysis. ${ }^{(17)}$ The second control dataset was acquired prospectively, to provide repeatability $\mathrm{T}_{1}$ measurements in volunteers scanned without contrast agent. For acquisition of both datasets, permission from the local Institutional Review Board was obtained and all volunteers signed informed consent. Furthermore, phantom measurements were performed to assess the limit of detection of gadobutrol by MRbased $\mathrm{T}_{1}$ mapping.

\section{ReMaRK Dataset}

The ReMaRK dataset consisted of nineteen healthy volunteers, aged 40 years or older and without history of kidney disease, who were included between March and November 2018 (Figure 1). Data of insufficient image quality were excluded. All subjects were examined twice on the same 3T MR system (Ingenia, Philips Healthcare, Best, the Netherlands; software release 5.3.1) with a 4-16 day interval between sessions. Blood was sampled prior to the first scan session to determine 


\section{ReMARK dataset Control dataset}

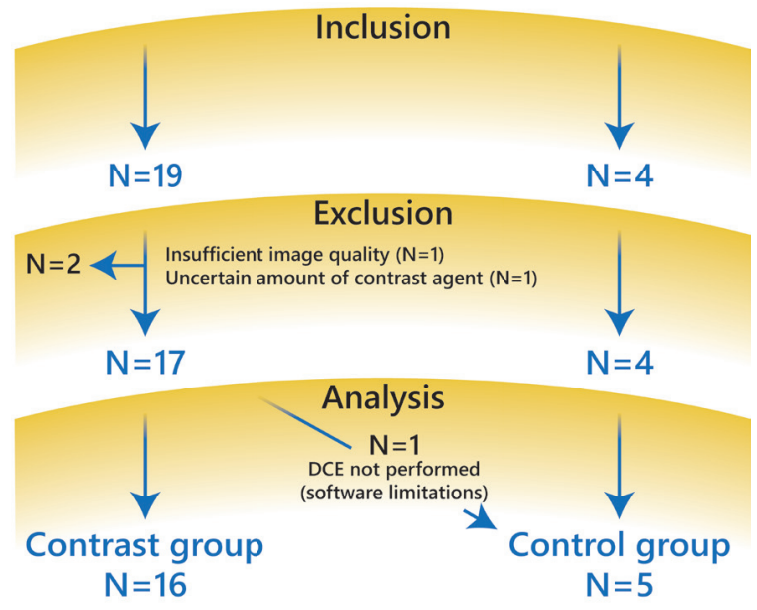

Figure 1. Flowchart of patient population for both datasets.

kidney function (creatinine and cystatin C). eGFR was calculated using the CKDEPI equation (Chronic Kidney Disease EPIdemiology collaboration, ${ }^{\left({ }^{18}\right)}$ ) using both cystatin $\mathrm{C}$ and creatinine. For cystatin $\mathrm{C}$ levels, the average of two measurements from a single plasma sample were used. All scan sessions for each volunteer were performed at the same time of the day, usually in the late afternoon. Subjects were asked to drink two liters of nonalcoholic liquids spread out over the day of the scan and to avoid salt- and protein-rich meals on the day of examination.

\section{Control Dataset}

The control dataset consisted of four healthy volunteers, aged 40 years or older and without history of kidney disease, who were included in June and July 2019 (Figure 1). Subjects were examined on the same $3 \mathrm{~T}$ MR system as described above using the same protocol for $\mathrm{T}_{1}$ mapping, with a 7 or 8 day interval. Again, scan sessions were performed in the late afternoon and with identical dietary instructions as described above.

\section{Imaging Protocol and Processing}

The ReMaRK dataset included, among other sequences, an initial survey, native $\mathrm{T}_{1}$ mapping, $\mathrm{T}_{1}$ weighted anatomical Dixon imaging and DCE renal perfusion imaging. The control dataset included only the survey and $\mathrm{T}_{1}$ mapping using the same protocol as the ReMaRK dataset.

The $\mathrm{T}_{1}$ map was acquired using an adiabatic spatially nonselective inversion pulse (hyperbolic secant) followed by a multislice readout, where the order of the slices was cycled after each repetition time to obtain multiple inversion time data for all slices. ${ }^{(19,20)}$ The scan was performed under synchronized breathing, where the 
Table 1. Scan parameters.

\begin{tabular}{|c|c|c|c|}
\hline & Survey & $T_{1}$ weighted Dixon & $T_{1} \operatorname{map}$ \\
\hline Sequence & $\begin{array}{l}\text { 2D balanced } \\
\text { GE }\end{array}$ & $\begin{array}{l}\text { 3D dual echo GE with } \\
\text { Dixon reconstruction of } \\
\text { water only images }\end{array}$ & $\begin{array}{l}\text { Inversion recovery with } 2 \mathrm{D} \\
\text { multislice GE readout }\end{array}$ \\
\hline Fast imaging & NA & $\mathrm{NA}$ & EPI \\
\hline Inversion pulse & NA & $\mathrm{NA}$ & Hyperbolic secant \\
\hline Inversion times (ms) & NA & $\mathrm{NA}$ & $\begin{array}{l}55 ; 253 ; 451 ; 649 ; 847 ; 1045 \\
1243 ; 1441 ; 1639 ; 1837 ; 2035\end{array}$ \\
\hline $\mathrm{TE}(\mathrm{ms})$ & 1.4 & $3.5 ; 4.6$ & 22 \\
\hline $\mathrm{TR}(\mathrm{ms})$ & 2.8 & $7 \cdot 5$ & 6500 \\
\hline Flip angle (degrees) & 25 & 8 & 90 \\
\hline Orientation & Coronal & Coronal oblique & Coronal oblique \\
\hline Slices & 10 & 35 & $11 \mathrm{a}$ \\
\hline Voxel size (mm) & $2 \times 1.56 \times 10$ & $1.5 \times 1.5 \times 3$ & $3 \times 3 \times 6$ \\
\hline FOV (mm) & $450 \times 450 \times 145$ & $320 \times 400 \times 70$ & $244 \times 244 \times 76$ \\
\hline Slice gap (mm) & 5 & o & 1 \\
\hline Acquisition time (s) & 31 & 19 & 72 \\
\hline
\end{tabular}

aThe first 4 subjects were scanned with 7 slices and 7 inversion times $(105 ; 373 ; 641 ; 909 ; 1177 ; 1445$; $1713 \mathrm{~ms}$ ); 2D: two dimensional; GE: gradient echo; EPI: echo planar imaging; TE: echo time;

TR: repetition time; NA: not applicable.

subjects breath between the readouts. Detailed scan parameters of the survey, the $\mathrm{T}_{1}$ map and the $\mathrm{T}_{1}$ weighted anatomical Dixon are provided in Table 1. During DCE imaging, which was performed last, a half-dose $(0.05 \mathrm{~mL} / \mathrm{kg})$ of gadobutrol was injected at a rate of $1 \mathrm{~mL} / \mathrm{min}$ followed by a $20 \mathrm{~mL}$ saline flush at the same rate.

Image processing was performed using in-house developed software in MatLab (MathWorks, Natick, MA, version 2015b). The center of both kidneys was identified manually. Next, a wide crop was made automatically around both kidneys such that each kidney could be processed separately. The remaining processing was performed independently for the left and right kidneys. To correct for respiratory motion, images were registered per slice in a group-wise manner using Elastix ${ }^{(21-23)}$. Registration was aimed to optimize alignment of the slices with varying inversion times in a region of interest encompassing the entire kidney. These regions of interest (ROIs) were generated by a semiautomated approach based on k-means clustering of the source data ${ }^{(24)}$. After registration, the entire cortex and medulla were segmented again using the clustering. Segmentations were checked, and corrected if necessary, by one observer (A.B.) with 5 years of experience in renal imaging. $\mathrm{T}_{1}$ relaxation times were calculated by a monoexponential nonlinear least squares fit on the magnitude data ${ }^{(25)}$ :

$$
|S|=k\left|1-2 \exp \left(-\frac{\mathrm{TI}}{\mathrm{T}_{1}}\right)\right|
$$


Here, $|\mathrm{S}|$ denotes the magnitude of the MR signal, $\mathrm{k}$ is a scaling factor including proton density and system gain and TI is the inversion time. The lowest datapoint acquired around the zero-crossing was excluded from the fit because of low a signalto-noise ratio. Apart from mean $\mathrm{T}_{1}$ values for cortical and medullary ROIs, also corticomedullary differentiation (CMD) was calculated as defined as medullary $\mathrm{T}_{1}$ minus cortical $\mathrm{T}_{1}$.

To approximate the contrast agent concentration $\mathrm{C}$, the following equation was used $^{(26)}$ :

$$
C \approx \frac{1}{r_{1}}\left(R_{1, \text { postcontrast }}-R_{1, \text { precontrast }}\right)=\frac{1}{r_{1}}\left(\frac{1}{T_{1, \text { postcontrast }}}-\frac{1}{T_{1, \text { precontrast }}}\right)
$$

Here, $\mathrm{r}_{1}$ denotes the $\mathrm{T}_{1}$ relaxivity of gadobutrol $\left(4.6 \mathrm{~mL} / \mathrm{mmol} / \mathrm{ms},{ }^{(27)}\right) . \mathrm{T}_{\mathrm{o}}$ calculate the proportion of the original dose retained in the kidneys, the kidney volume was required. This was measured by manual segmentation on $\mathrm{T}_{1}$ weighted anatomical Dixon scans by the same observer (A.B.). To be able to roughly compare the control and gadolinium group in terms of renal health, for both groups kidney length was measured on the survey.

\section{Phantom Study}

The aim of the phantom study was to find the detection limit, or lowest gadobutrol concentration which could be detected using $\mathrm{T}_{1}$ mapping. The phantom is depicted in Figure 2. An insulated cylindrical container was filled with $2 \mathrm{~L}$ of $0.9 \%$ saline. Four different concentrations of gadobutrol (100, 10, 1 and $0.1 \mathrm{nmol} / \mathrm{mL}$ dissolved in $0.9 \%$ saline) were obtained by serial dilution. Four $50 \mathrm{~mL}$ tubes containing these solutions were placed inside the container. The container was placed in the scanner parallel to the orientation of the $\mathrm{B}_{\mathrm{o}}$ field. All parts of the phantom were placed in a $38{ }^{\circ} \mathrm{C}$ stove 3 hours in advance and the phantom was taken out of the stove just before the MRI exam to assure it would be at approximately $37^{\circ} \mathrm{C}$ during acquisition. The same $\mathrm{T}_{1}$ mapping protocol was used as for the human studies, but the slice orientation was axial instead of coronal. The phantom study was performed on the same $3 \mathrm{~T} \mathrm{MR}$ system as described above.

Generally, a signal at the detection limit $S_{d e t}$ can be calculated as follows ${ }^{(28)}$ :

$$
S_{\text {det }}-S_{\text {blank }}=1.645 *\left(s d_{\text {blank }}+s d_{\text {low }}\right)
$$



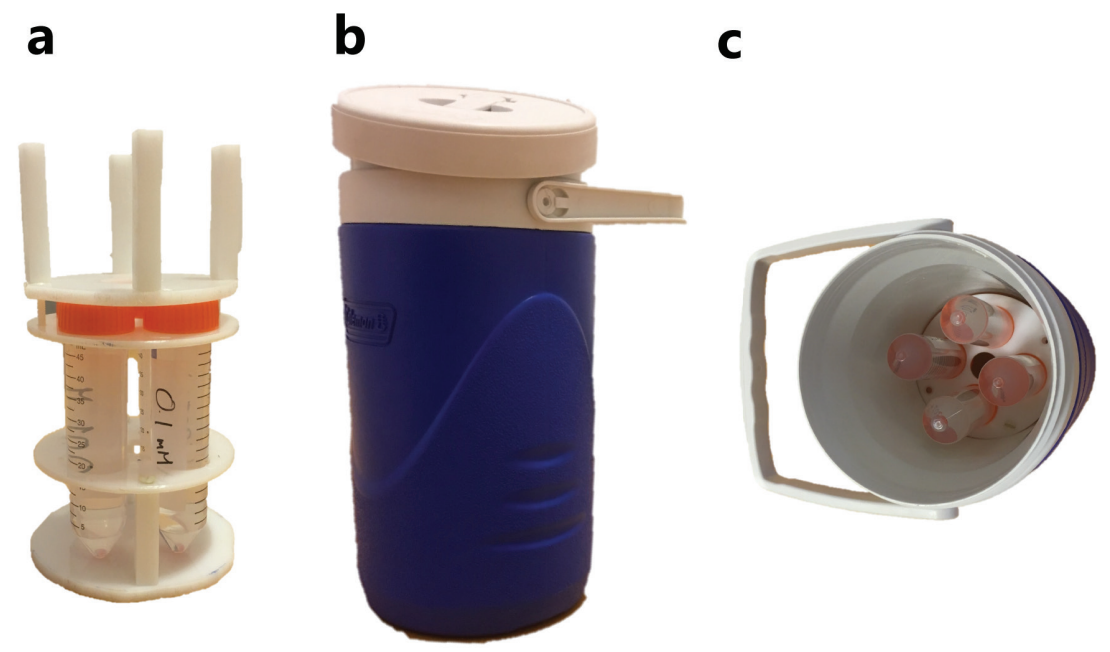

Figure 2. Setup of phantom experiment. a Four different solutions of gadobutrol were prepared in $50 \mathrm{~mL}$ tubes an placed in a holder. $\mathbf{b}$ The insulating container filled with $0.9 \%$ saline. c The holder inside the container.

Where $S_{\text {blank }}$ is the mean blank (saline) $\mathrm{T}_{1}$ and $s d_{\text {blank }}$ and $s d_{\text {low }}$ are the standard deviation of the blank and the low concentration $\mathrm{T}_{1}$, respectively. In combination with equation 2 the detection limit can be calculated.

\section{Statistical Analysis}

Data are reported as mean \pm standard deviation or median (interquartile range, IQR) where appropriate. To compare the $\mathrm{T}_{1}$ difference between the scan sessions $\left(\Delta \mathrm{T}_{1}\right)$ between the gadolinium and control group, a Mann-Whitney-U test was used. Linear regression was performed to investigate the dependency of $T_{1}$ difference on time between examinations and eGFR (not corrected for body surface area). Correlation between the $\mathrm{T}_{1}$ difference and eGFR was assessed only in the subgroup of volunteers scanned at an interval of precisely 7 days to avoid the influence of different washout periods. A p-value of 0.05 was considered statistically significant. Statistical analyses were performed in $\mathrm{R}$ version 3.4.4.(29)

\section{Results}

\section{Clinical Study}

A flow chart summarizing the study population for both the ReMaRK $(\mathrm{N}=19)$ and control $(\mathrm{N}=4)$ dataset is shown in Figure 1. In the ReMaRK dataset, data of two subjects had to be excluded because of insufficient image quality $(n=1)$ and an uncertain amount of contrast agent due to problems with the intravenous access $(n=1)$. For analysis, a gadolinium and a control group were formed. The gadolinium 
Table 2. Baseline characteristics of analyzed study population. Data are reported as mean \pm standard deviation or median (interquartile range).

\begin{tabular}{|c|c|c|}
\hline & Gadolinium group & Control group \\
\hline $\mathrm{N}$ & 16 & 5 \\
\hline Median age [year] & $50(45-58)$ & $52(48-59)$ \\
\hline Median eGFR $\left[\mathrm{mL} / \mathrm{min} / 1.73 \mathrm{~m}^{2}\right]$ & $98(88-102)$ & NA \\
\hline Median scan interval [days] & $7(7-7)$ & $7(7-7)$ \\
\hline Median cortical $\mathrm{T}_{1}$ & $1516(1488-1548)$ & $1531(1479-1555)$ \\
\hline Median medullary $\mathrm{T}_{1}$ & $1862(1837-1900)$ & $1864(1810-1917)$ \\
\hline Median CMD & $350(332-376)$ & $334(320-373)$ \\
\hline Mean cortical volume & $103 \pm 20$ & NA \\
\hline Mean medullary volume & $34 \pm 6$ & NA \\
\hline Median kidney length* & $10.6(10.1-11.0)$ & $10.5(9.4-10.8)$ \\
\hline
\end{tabular}

*corrected for body length (kidney length / body length * $175 \mathrm{~cm}$ ); NA: not available; CMD: corticomedullary differentiation, defined as medullary $\mathrm{T}_{1}$ minus cortical $\mathrm{T}_{1}$.

group consisted solely of subjects of the ReMaRK dataset. The control group consisted of four subjects included in this group and one subject of the ReMaRK dataset who did not receive contrast agent. Therefore, the gadolinium and the control group consisted of 16 and 5 subjects, respectively (Figure 1). All volunteers in the gadolinium group had an eGFR in the normal range. Baseline characteristics of both groups are provided in table 2. Renal health in terms of kidney length and CMD was comparable in both groups. CMD in the $\mathrm{T}_{1}$ map was slightly lower in the control group but the difference was not statistically significant $(\mathrm{p}=0.42)$. An example of a calculated $\mathrm{T}_{1}$ map including source data and segmentation of cortex and medulla is shown in Figure 3.

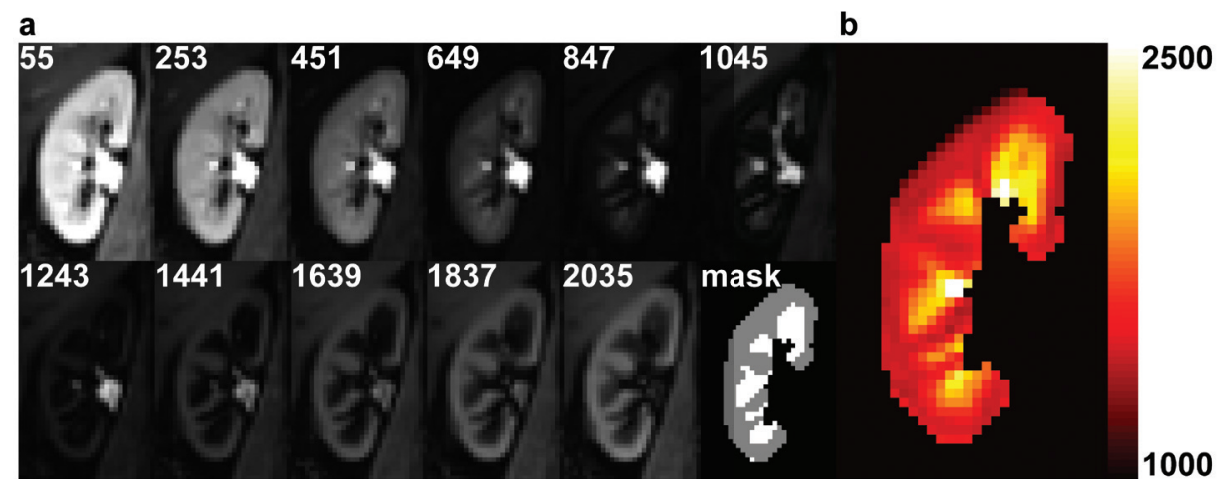

Figure 3. Example images of the right kidney from a healthy volunteer obtained at the first scan session; a $\mathrm{T}_{1}$ source images at multiple inversion times (in ms) after motion correction and the masks of the cortical and medullary segmentation; $\mathbf{b}$ Calculated corresponding $\mathrm{T}_{1}$ map. The color bar indicates $\mathrm{T}_{1}$ relaxation time in ms. Cortex and medulla can easily be discriminated thanks to the higher $\mathrm{T}_{1}$ in medulla compared to cortex. 
a

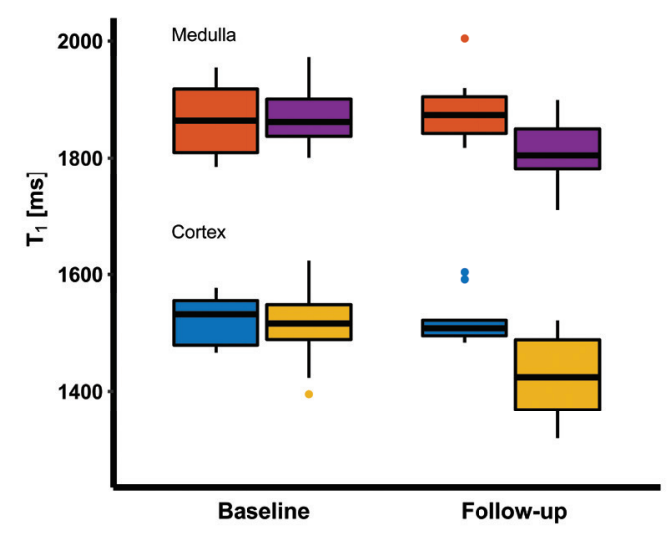

b

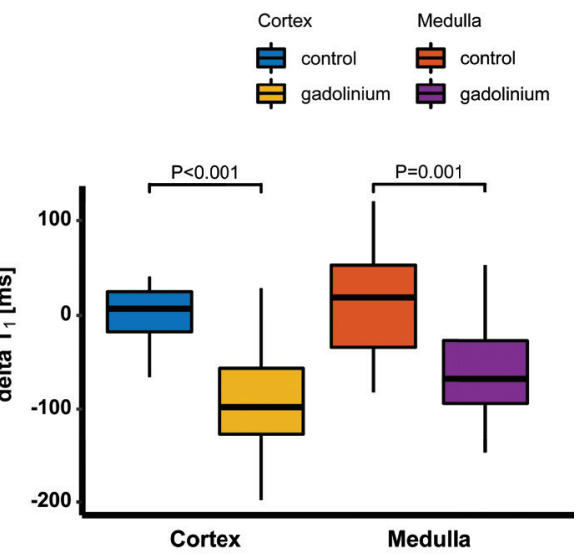

Figure 4. a Boxplots summarizing the $\mathrm{T}_{1}$ values in the gadolinium $(\mathrm{n}=16)$ and control group $(n=5)$ at baseline and follow-up; $\mathbf{b}$ boxplots of the $\Delta \mathrm{T}_{1}$ for cortex and medulla and both for the gadolinium and control group. $\Delta \mathrm{T}_{1}$ in the gadolinium group differed significantly from $\Delta \mathrm{T}_{1}$ in the control group, both in cortex $(\mathrm{p}<0.001)$ and medulla $(\mathrm{p}=0.001)$.

The cortical and medullary $\mathrm{T}_{1}$ values at baseline and follow-up are summarized in Figure 4a, separately for the gadolinium and control group. The difference in $\mathrm{T}_{1}$ between baseline and follow-up $\left(\Delta \mathrm{T}_{1}\right)$ differed significantly between the both groups (Figure $4 \mathrm{~b}$ ). In the cortex, the median $\Delta \mathrm{T}_{1}$ was -98 (IQR: -127- -57) in the gadolinium group versus 7 (IQR: $-18-25$ ) $\mathrm{ms}$ in the control group ( $\mathrm{p}<0.001)$. In the medulla, median $\Delta \mathrm{T}_{1}$ in the gadolinium and control group were -68 (IQR: -94 - -27) ms versus 19 (IQR: $-34-53) \mathrm{ms}$, respectively ( $\mathrm{p}=0.001)$.

Assuming the $\Delta \mathrm{T}_{1}$ in the gadolinium group is caused by remaining contrast agent, the renal gadobutrol concentration can be estimated. For subjects scanned at a scan interval of 7 days $(\mathrm{n}=12)$, a median $\Delta \mathrm{T}_{1}$ of -98 (IQR: $\left.-120--66\right) \mathrm{ms}$ and -69 (IQR: -94 - -46) ms was measured for cortex and medulla, respectively. This corresponds to a concentration of $\sim 8 \mathrm{nmol} / \mathrm{mL}$ in the cortex and $\sim 4 \mathrm{nmol} / \mathrm{mL}$ in the medulla, compared to an expected plasma concentration of $3^{*} 10^{-23} \mathrm{nmol} / \mathrm{mL}$ in the conventional two-phase pharmacokinetic model. Assuming a renal tissue density of $1 \mathrm{~g} / \mathrm{mL}$, this corresponds to a concentration of $8 \mathrm{nmol} / \mathrm{g}$ (cortex) and $4 \mathrm{nmol} / \mathrm{g}$ (medulla). Using the average cortical and medullary volumes of all 16 subjects in the gadolinium group (103 $\mathrm{mL}$ and $34 \mathrm{~mL}$, respectively) and assuming a dose of 4 mmol (mean weight in this subgroup was $79 \mathrm{~kg}$ ), we can calculate that $0.05 \%$ of the original dose remained after 7 days in the kidneys (expected based on two-phase pharmacokinetics $10^{-26} \%$ ).

It is expected that $\Delta \mathrm{T}_{1}$ decreases and approaches zero with increasing scan interval if caused by slow elimination of gadobutrol. Therefore, $\Delta \mathrm{T}_{1}$ dependence on the time interval between scan sessions (scan interval) was investigated in the gadolinium group. Four of the 16 subjects were scanned with intervals of 4, 5, 8 and 
16 days for logistical reasons. Linear regression with the scan interval as predicting variable and $\Delta \mathrm{T}_{1}$ as dependent variable (Figure 5 ) yielded a regression coefficient of $16.5 \mathrm{~ms} /$ day $\left(\mathrm{R}^{2} \mathrm{0.49}, \mathrm{p}<0.001\right)$ for the cortex and $11.5 \mathrm{~ms} /$ day $\left(\mathrm{R}^{2} \mathrm{0.30}, \mathrm{p}<0.001\right)$ in the medulla.

Furthermore, in the subset of subjects in the gadolinium group scanned at an interval of 7 days $(\mathrm{n}=12) \Delta \mathrm{T}_{1}$ correlated significantly with eGFR in the medulla $\left(\mathrm{R}^{2} \mathrm{0.25}, \mathrm{p}=0.008\right.$, Figure 6b), but not in the cortex $\left(\mathrm{R}^{2} \mathrm{0.11}, \mathrm{p}=0.07\right.$, Figure 6a).

a

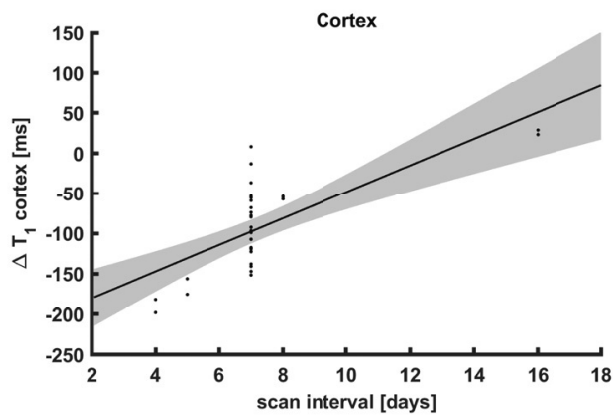

b

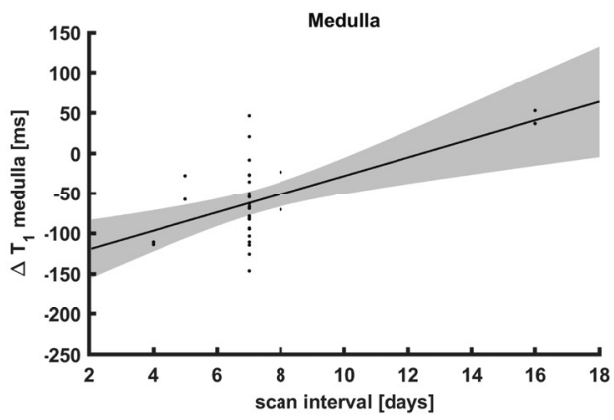

Figure 5. Regression analysis of the dependence of $\Delta \mathrm{T}_{1}$ on scan interval in the gadolinium group $(\mathrm{n}=16)$. Shaded areas denote the $95 \%$ confidence interval of the regression line; a Cortical $\Delta \mathrm{T}_{1}$ values, $\mathrm{R}^{2} 0.49, \mathrm{p}<0.001 ; \mathbf{b}$ Medullary $\Delta \mathrm{T}_{1}$ values, $\mathrm{R}^{2} 0.30, \mathrm{p}<0.001$.
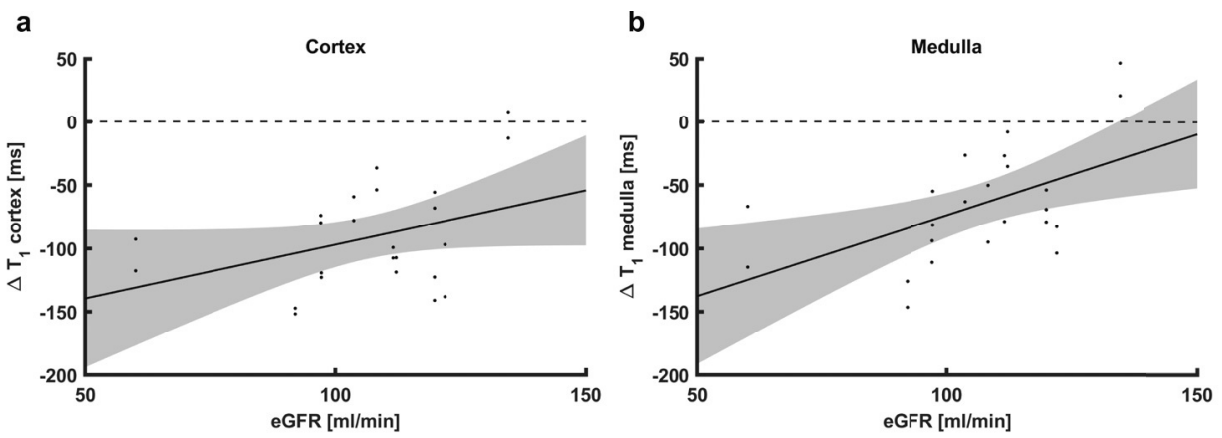

Figure 6. Scatter plots of $\Delta \mathrm{T}_{1}$ versus eGFR (not corrected for body surface area), for subjects in the gadolinium group scanned with a seven-day interval only $(\mathrm{n}=12)$. Shaded areas denote the $95 \%$ confidence interval of the regression line; a For cortical $\Delta \mathrm{T}_{1}$, the correlation was not significant; b Medullary $\Delta \mathrm{T}_{1}$ correlated significantly with eGFR, $\left(\mathrm{R}^{2} 0.25, \mathrm{p}=0.008\right)$. eGFR: estimated glomerular filtration rate.

\section{Phantom Study}

$\mathrm{T}_{1}$ maps of the two middle slices of the phantom are shown in Figure 7a and b. Masks were drawn as shown in Figure $7 \mathrm{~b}$, avoiding susceptibility artifacts caused by air bubbles and partial volume voxels. In Figure $7 \mathrm{c}$, the $\mathrm{T}_{1}$ distributions of the saline 
$0.9 \%$ and gadobutrol $0.1,1$ and $10 \mathrm{nmol} / \mathrm{mL}$ are shown. Fitting of an exponential model to these data yields a relaxivity of $3.1 \mathrm{~mL} / \mathrm{mmol} / \mathrm{ms}$ of gadobutrol in saline, which is slightly lower than the $\mathrm{r}_{1}$ in blood/plasma of $4.6 \mathrm{~mL} / \mathrm{mmol} / \mathrm{ms}^{(27)} \mathrm{Mean}_{1}$ of the $100 \mathrm{nmol} / \mathrm{mL}$ solution was $1644 \pm 19 \mathrm{~ms}$ (not shown in Figure $7 \mathrm{c}$ ). Using the mean and standard deviations of saline $0.9 \%(3311 \pm 36 \mathrm{~ms})$ and the $1 \mathrm{nmol} / \mathrm{mL}$ solution ( $3253 \pm 14 \mathrm{~ms}$ ), we find the $\mathrm{T}_{1}$ at the detection limit to be $3229 \mathrm{~ms}$, which corresponds to a concentration of $2.5 \mathrm{nmol} / \mathrm{ml}$ (or $2.5 \mathrm{nmol} / \mathrm{g}$ assuming a tissue density of $1 \mathrm{~g} / \mathrm{mL}$ ) in saline. The detection limit in kidneys probably differs somewhat, given the difference in relaxivity and $\mathrm{T}_{1}$.
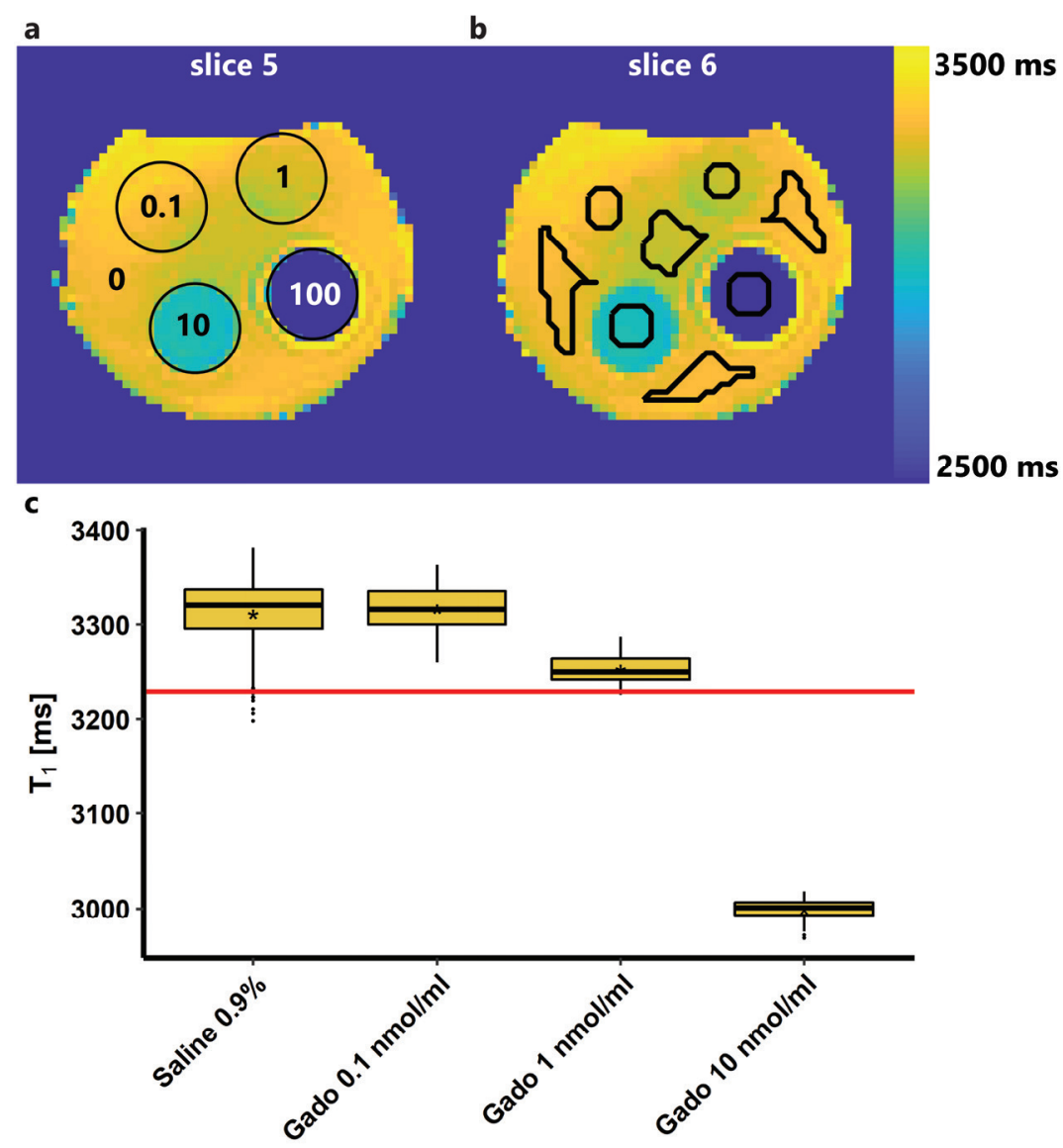

Figure 7. Results of phantom experiment; $\mathbf{a}$ and $\mathbf{b} \mathrm{T}_{1}$ maps of the two middle slices of the phantom. $T_{1}$ values across the phantom are not completely homogeneous due to inhomogeneity of the magnetic field; a The different tubes are denoted with circles and the gadobutrol concentrations are given in $\mathrm{nmol} / \mathrm{mL}$. The tubes are surrounded by saline $0.9 \%$, denoted with a o (o nmol/mL gadobutrol); b The regions of interests (black lines) were drawn manually, carefully avoiding partial volume and susceptibility artefacts; $\mathbf{c}$ Boxplots of the $\mathrm{T}_{1}$ distribution inside each region of interest. The mean is denoted with an asterisk $(*)$. The red line denotes the detection limit, it corresponds to a concentration of $2.5 \mathrm{nmol} / \mathrm{mL}$ gadobutrol. 


\section{Discussion}

In this study on renal $T_{1}$ measurements before and $\sim 7$ days after gadobutrol administration in healthy volunteers we observed a systematic negative bias in the second $\mathrm{T}_{1}$ measurement in the gadolinium group. Since this difference was absent in the control group, we consider the presence of traces of remaining contrast agent from the previous visit the only feasible explanation for this finding. Since the second scan was performed $\sim 7$ days after the first, this is not consistent with conventional two-phase pharmacokinetics ${ }^{(16)}$

The study was designed to rule out systematic differences between the first and second examination. All volunteers were scanned on the same scanner twice at the same time of the day. Physiological variation was minimized by roughly controlling diet and hydration. Moreover, if the observed differences were due to physiological variations, one would expect random and not systematic variation.

Apart from the absence of a $\mathrm{T}_{1}$ bias in the control group, the following reasons support the hypothesis of remaining contrast agent from the previous administration. First, GBCAs induce a decrease in $\mathrm{T}_{1}$ relaxation time, which we found. Second, a decrease of the $\mathrm{T}_{1}$ bias with time was demonstrated, possibly indicating gradual excretion. Third, it has been shown on retrospective data that GBCA elimination fits a three phase model. ${ }^{\left({ }^{15}\right)}$ In this model, phases of distribution and elimination are followed by a slow residual elimination phase from a so called deep compartment, probably bone, with time constants ranging from 6-102 hours. ${ }^{(15)}$ Fourth, gadolinium deposition in the kidneys has been reported in animals, see below. Last, as a macrocyclic agent gadobutrol is relatively stable, and dissociation from its chelate is neither expected nor shown in either humans or animals. There is no clear evidence of cytotoxic changes induced by gadolinium deposits. ${ }^{\left({ }^{\circ}\right)}$ Furthermore, with cytotoxicity, $\mathrm{T}_{1}$ increase would be expected, due to edema of inflamed tissue. Therefore, we think that the negative $\Delta \mathrm{T}_{1}$ can be attributed to small amounts of gadobutrol rather than to gadolinium-induced cytotoxic tissue changes.

Renal deposition of gadobutrol has been shown in rats exposed to the equivalent of 80 human intravenous doses of various GBCAs over a 26-day period. ${ }^{(31)}$ After one recovery week, GBCA-exposed rats had elevated levels of gadolinium in renal, hepatic, splenic and to a lesser extent neural tissue. This was more pronounced for linear than macrocyclic agents. Histological changes were only seen in the kidney, demonstrating diffuse epithelial vacuolization in the cortex with preserved glomerular architecture. For the (macrocyclic) agent gadoteridol, also signs of renal injury were seen despite lower tissue concentrations compared to gadobutrol and the linear agents. Another study exposed rats during a 5-week period to the equivalent of 20 human doses of macrocyclic GBCAs. ${ }^{(32)}$ After a 4-week recovery 
period, gadolinium was detected in kidneys, femur and neural tissues but not in liver. Mean renal gadobutrol concentration was $139 \mathrm{nmol} / \mathrm{g}$ tissue. In comparison, the concentration of $\sim 4-8 \mathrm{nmol} / \mathrm{g}$ tissue as found here seems reasonable given the $40-$ fold lower dose. The phantom experiment indicates that gadobutrol concentrations in this range are detectable using MR-based $\mathrm{T}_{1}$ mapping. In both kidneys combined, in the gadolinium group around $\sim 2 \mu \mathrm{mol}$ of gadobutrol remains after 7 days, while the prescribing information states that even in subjects with renal function impairment (eGFR $30-80 \mathrm{~mL} / \mathrm{min}$ ) the complete dose should be recovered in urine within 72 hours. ${ }^{(16)}$ Therefore, our findings support the existence of a slow residual elimination phase for gadobutrol. ${ }^{(15)}$

$\Delta \mathrm{T}_{1}$ was highest in the cortex. Although this is consistent with the report of cortical histological changes found mainly in the cortex of GBCA exposed rats, ${ }^{\left({ }^{11}\right)}$ the gadobutrol dose we used was 160 -fold lower compared to this rodent study. Conversely, the lower medullary $\Delta \mathrm{T}_{1}$ correlated strongly with eGFR, while this correlation was not significant in the cortex. Why this relation is more pronounced in the medulla is unclear.

A limitation of this study is that eGFR in the control group was not available. Since all subjects in this group were healthy without any history of kidney disease, high blood pressure or diabetes, their eGFR was assumed to be in the normal range. As a substitute measure for renal health, CMD in terms of $\mathrm{T}_{1}$ and kidney length as measured in the scout scans were reported. Kidney length was measured on the survey images and is likely an underestimation since the survey was not necessarily aligned with the long axis of the kidney. Since the subjects in the control group did not receive gadobutrol it is unlikely that the repeated $\mathrm{T}_{1}$ measurements were influenced by their kidney function. Between the examinations, behavior of the subjects was neither restricted nor documented. Heavy exercise for example could potentially induce hematuria and consequently a decrease in $\mathrm{T}_{1}$. As the sample size of this study was limited, these preliminary results should be confirmed in a larger study, ideally also comparing multiple GBCAs. Furthermore, future work should include repeated $\mathrm{T}_{1}$ measurements in the same individual, possibly revealing a near-exponential decay of $\mathrm{T}_{1}$ illustrating the gradual elimination of contrast agent. We chose not to perform an exponential analysis on our data because of the uneven distribution of timepoints and since only two measurements per subject were available. Longer term ( $\geq 2$ weeks) follow up measurements can indicate when the contrast agent concentration drops below the detection limit. Ideally, plasma and urine collections should be available to detect and quantify gadolinium traces, possibly using inductively coupled plasma mass spectrometry. Without tissue samples, it cannot be ruled out that a process other than slow clearance of gadobutrol is responsible for the $\mathrm{T}_{1}$ difference. Last, the detection limit for gadobutrol in the phantom experiment likely differs somewhat 
from the actual detection limit in the kidney, due to the lower relaxivity of gadobutrol in saline and the long $\mathrm{T}_{1}$ of saline compared to renal tissue.

In conclusion, we found evidence of the prolonged presence of small amounts of gadobutrol in renal tissue after a single administration of GBCA in subjects with normal renal function, probably based on delayed elimination. Even in this healthy population, elimination delay increased with decreasing kidney function. This study adds to existing reports of gadolinium retention in brain, bone and skin. However, it is important to underscore that to date there are no reports of adverse effects of macrocyclic contrast agents in subjects with normal renal function. Nevertheless, our data suggest that it would be wise to perform studies on long-term safety. These studies should at least include renal endpoints, especially in patients with (mildly) decreased eGFR or patients receiving multiple GBCA administrations. Furthermore, little is known about the biodistribution and late pharmacokinetics of GBCAs in humans for the small fraction that remains after the initial phases of distribution and (renal) clearance. MRI based $\mathrm{T}_{1}$ mapping may prove to be a valuable tool to monitor GBCA biodistribution over time in vivo.

\section{Acknowledgements}

The authors thank Jeanine Prompers, Erik Huijing and Katharina Milde for use of the phantom that they have developed. 


\section{References}

1. Grobner T. Gadolinium--a specific trigger for the development of nephrogenic fibrosing dermopathy and nephrogenic systemic fibrosis? Nephrol Dial Transplant. 2006;21(4):1104-8.

2. Marckmann P, Skov L, Rossen K, Dupont A, Damholt MB, Heaf JG, et al. Nephrogenic systemic fibrosis: suspected causative role of gadodiamide used for contrast-enhanced magnetic resonance imaging. J Am Soc Nephrol. 2006;17(9):2359-62.

3. McDonald RJ, Levine D, Weinreb J, Kanal E, Davenport MS, Ellis JH, et al. Gadolinium Retention: A Research Roadmap from the 2018 NIH/ACR/RSNA Workshop on Gadolinium Chelates. Radiology. 2018;289(2):517-34.

4. Attari H, Cao Y, Elmholdt TR, Zhao Y, Prince MR. A Systematic Review of 639 Patients with Biopsy-confirmed Nephrogenic Systemic Fibrosis. Radiology. 2019;292(2):37686.

5. Kanda T, Ishii K, Kawaguchi H, Kitajima K, Takenaka D. High signal intensity in the dentate nucleus and globus pallidus on unenhanced T1-weighted MR images: relationship with increasing cumulative dose of a gadolinium-based contrast material. Radiology. 2014;270(3):834-41.

6. McDonald RJ, McDonald JS, Kallmes DF, Jentoft ME, Murray DL, Thielen KR, et al. Intracranial Gadolinium Deposition after Contrast-enhanced MR Imaging. Radiology. 2015;275(3):772-82.

7. Roberts DR, Lindhorst SM, Welsh CT, Maravilla KR, Herring MN, Braun KA, et al. High Levels of Gadolinium Deposition in the Skin of a Patient With Normal Renal Function. Invest Radiol. 2016;51(5):280-9.

8. Olchowy C, Cebulski K, Lasecki M, Chaber R, Olchowy A, Kalwak K, et al. The presence of the gadolinium-based contrast agent depositions in the brain and symptoms of gadolinium neurotoxicity - A systematic review. PLoS One. 2017;12(2):e0171704.

9. Behzadi AH, Zhao Y, Farooq Z, Prince MR. Immediate Allergic Reactions to Gadolinium-based Contrast Agents: A Systematic Review and Meta-Analysis. Radiology. 2018;286(2):471-82.

10. Soyer P, Dohan A, Patkar D, Gottschalk A. Observational study on the safety profile of gadoterate meglumine in 35,499 patients: The SECURE study. J Magn Reson Imaging. 2017;45(4):988-97.

11. Amet S, Launay-Vacher V, Clement O, Frances C, Tricotel A, Stengel B, et al. Incidence of nephrogenic systemic fibrosis in patients undergoing dialysis after contrastenhanced magnetic resonance imaging with gadolinium-based contrast agents: the Prospective Fibrose Nephrogenique Systemique study. Invest Radiol. 2014;49(2):10915.

12. McWilliams RG, Frabizzio JV, De Backer AI, Grinberg A, Maes BD, Zobel BB, et al. 
Observational study on the incidence of nephrogenic systemic fibrosis in patients with renal impairment following gadoterate meglumine administration: the NSsaFe study. J Magn Reson Imaging2019.

13. Aime S, Caravan P. Biodistribution of gadolinium-based contrast agents, including gadolinium deposition. J Magn Reson Imaging. 2009;30(6):1259-67.

14. Staks T, Schuhmann-Giampieri G, Frenzel T, Weinmann HJ, Lange L, Platzek J. Pharmacokinetics, dose proportionality, and tolerability of gadobutrol after single intravenous injection in healthy volunteers. Invest Radiol. 1994;29(7):709-15.

15. Lancelot E. Revisiting the Pharmacokinetic Profiles of Gadolinium-Based Contrast Agents: Differences in Long-Term Biodistribution and Excretion. Invest Radiol. 2016;51(11):691-700.

16. Bayer Healthcare Pharmaceuticals. HIGHLIGHTSOF PRESCRIBINGINFORMATION: US Food and Drug Administration; 2011 [updated March 2011. Available from: https:// www.accessdata.fda.gov/drugsatfda_docs/label/2011/201277sooolbl.pdf.

17. de Boer A, Harteveld AA, Pieters TT, Blankestijn PJ, Bos C, Froeling M, et al. Decreased native renal T1 up to one week after gadobutrol administration in healthy volunteers. $\mathrm{J}$ Magn Reson Imaging2019.

18. Inker LA, Eckfeldt J, Levey AS, Leiendecker-Foster C, Rynders G, Manzi J, et al. Expressing the CKD-EPI (Chronic Kidney Disease Epidemiology Collaboration) cystatin C equations for estimating GFR with standardized serum cystatin $\mathrm{C}$ values. Am J Kidney Dis. 2011;58(4):682-4.

19. Ordidge RJ, Gibbs P, Chapman B, Stehling MK, Mansfield P. High-speed multislice T1 mapping using inversion-recovery echo-planar imaging. Magn Reson Med. 1990;16(2):238-45.

20. Clare S, Jezzard P. Rapid T(1) mapping using multislice echo planar imaging. Magn Reson Med. 2001;45(4):630-4.

21. Klein S, Staring M, Murphy K, Viergever MA, Pluim JP. elastix: a toolbox for intensitybased medical image registration. IEEE Trans Med Imaging. 2010;29(1):196-205.

22. Shamonin DP, Bron EE, Lelieveldt BP, Smits M, Klein S, Staring M. Fast parallel image registration on CPU and GPU for diagnostic classification of Alzheimer's disease. Front Neuroinform. 2013;7:50.

23. Huizinga W, Poot DH, Guyader JM, Klaassen R, Coolen BF, van Kranenburg M, et al. PCA-based groupwise image registration for quantitative MRI. Med Image Anal. 2016;29:65-78.

24. de Boer A, Leiner T, Vink EE, Blankestijn PJ, van den Berg CAT. Modified dixonbased renal dynamic contrast-enhanced MRI facilitates automated registration and perfusion analysis. Magn Reson Med. 2018;80(1):66-76.

25. Brown RW, Cheng YCN, Haacke EM, Thompson MR, Venkatesan R. Introductory Signal Acquisition Methods: Free Induction Decay, Spin Echoes, Inversion Recovery, and Spectroscopy. Magnetic Resonance Imaging Physical Principles and Sequence 
Design. 2nd ed. Hoboken, New Jersey, USA: John Wiley \& Sons, Inc; 2014. p. 113-36.

26. Brown RW, Cheng YCN, Haacke EM, Thompson MR, Venkatesan R. Signal, Contrast, and Noise. Magnetic Resonance Imaging Physical Principles and Sequence Design. 2nd ed. Hoboken, New Jersey, USA: John Wiley \& Sons, Inc; 2014. p. 325-70.

27. Szomolanyi P, Rohrer M, Frenzel T, Noebauer-Huhmann IM, Jost G, Endrikat J, et al. Comparison of the Relaxivities of Macrocyclic Gadolinium-Based Contrast Agents in Human Plasma at 1.5, 3, and 7 T, and Blood at 3 T. Invest Radiol. 2019;54(9):559-64.

28. Armbruster DA, Pry T. Limit of blank, limit of detection and limit of quantitation. Clin Biochem Rev. 2008;29 Suppl 1:S49-52.

29. R Development Core Team. R: A language and environment for statistical computing. R Foundation for Statistical Computing. Vienna, Austria: R Foundation for Statistical Computing; 2018.

30. Blumfield E, Swenson DW, Iyer RS, Stanescu AL. Gadolinium-based contrast agents review of recent literature on magnetic resonance imaging signal intensity changes and tissue deposits, with emphasis on pediatric patients. Pediatr Radiol. 2019;49(4):44857.

31. McDonald RJ, McDonald JS, Dai D, Schroeder D, Jentoft ME, Murray DL, et al. Comparison of Gadolinium Concentrations within Multiple Rat Organs after Intravenous Administration of Linear versus Macrocyclic Gadolinium Chelates. Radiology. 2017;285(2):536-45.

32. Bussi S, Coppo A, Botteron C, Fraimbault V, Fanizzi A, De Laurentiis E, et al. Differences in gadolinium retention after repeated injections of macrocyclic MR contrast agents to rats. J Magn Reson Imaging. 2018;47(3):746-52. 



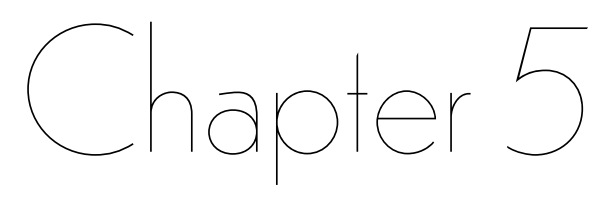

The Effect of Renal Denervation on Kidney $O$ xygenation as Determined by BOLD MRI in Patients With Hypertension

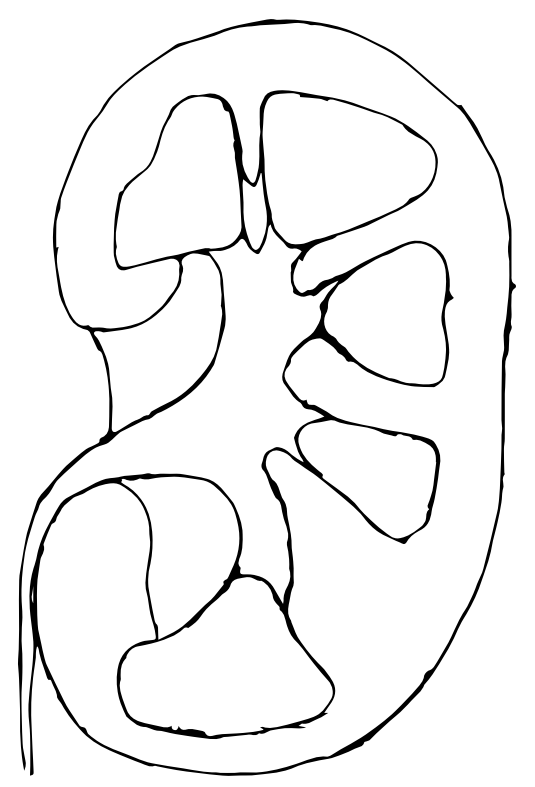

\section{Author contributions}

Vink EE, Boer A, Verloop WL, Spiering W, Voskuil M, Vonken E, Hoogduin JM, Leiner T, Bots ML, Blankestijn PJ

$$
\text { Published in }
$$

Eur Radiol 2015; doi:10.1007/s00330-014-3583-1 


\section{Abstract}

Background Renal denervation (RDN) is a promising therapy for resistant hypertension. RDN is assumed to decrease sympathetic activity, resulting in altered sodium handling by the kidneys and a decrease in peripheral vascular resistance. Consequently, RDN can potentially increase renal oxygenation. Blood oxygen level dependent MRI (BOLD-MRI) provides a non-invasive tool to determine renal oxygenation in humans. Increased $\mathrm{R}_{2}{ }^{*}$-levels imply decreased renal oxygen levels. The aim of the current study was to investigate the effect of RDN on renal oxygenation as determined by BOLD-MRI.

Methods Patients with resistant hypertension or the inability to follow a stable drug regimen due to unacceptable side effects were included. BOLD-MRI was performed before and 12 months after RDN. 27 patients were imaged on 3.0T and 19 on $1.5 \mathrm{~T}$ clinical MRI systems. Since most drugs influence BOLD-MRI, anti-hypertensive medication was temporarily stopped before MRI when considered safe. Blood pressure (BP) was assessed at baseline and follow-up using ambulatory 24-hr BP monitoring.

Results 54 patients were included, 46 patients (23 males, mean age 57 ) completed the study. Mean 24-hr BP changed from 163( \pm 20$) / 98( \pm 14) \mathrm{mm} \mathrm{Hg}$ to $154( \pm 22) / 92( \pm 13)$ $\mathrm{mm} \mathrm{Hg}(\mathrm{p}=0.001$ and $\mathrm{p}<0.001)$. eGFR did not change after RDN (77 (18) vs 79 (20) $\left.\mathrm{mL} / \mathrm{min} / 1.73 \mathrm{~m}^{2} \mathrm{p}=0.13\right)$. RDN did not affect renal oxygenation (1.5T: cortical $\mathrm{R}_{2}{ }^{*}$ : $12.5( \pm 0.9)$ vs $12.5( \pm 0.9) \mathrm{s}^{-1} \mathrm{p}=0.94$, medulary $\mathrm{R}_{2}^{*}: 19.6( \pm 1.7)$ vs $19.3(1.4) \mathrm{s}^{-1} \mathrm{p}=0.40$, $3 \mathrm{~T}$ : cortical $\mathrm{R}_{2}^{*}$ : $18.1( \pm 0.8)$ vs $17.8( \pm 1.2) \mathrm{s}^{-1} \mathrm{p}=0.47$, medullary $\mathrm{R}_{2}^{*}: 27.4( \pm 1.9)$ vs $\left.26.7( \pm 1.8) \mathrm{s}^{-1} \mathrm{p}=0.19\right)$. In those who stopped medication twice the results were the same. Furthermore, in responders of RDN (decrease in daytime SBP $\geq 5 \mathrm{~mm} \mathrm{Hg}$ ), renal oxygenation did not change.

Conclusion The current study shows that RDN does not lead to changes in renal oxygenation one year after RDN as determined by BOLD-MRI. 


\section{Introduction}

Renal hypoxia is thought to both be a result as well as a cause of hypertension, potentially inducing a vicious cycle. In several animal models of hypertension, cortical as well as medullary oxygenation is lower in hypertensive animals compared to controls..$^{(1)}$ This can be a result of increased sodium retention, a process requiring active sodium transport, which might lead to increased tubular oxygen consumption. Moreover, the renin angiotensin system (RAAS) and the sympathetic nervous system (SNS) are activated in hypertension, both influencing renal perfusion and oxygenation.

Animal studies show that renal ischemia triggers activation of the SNS. Even a small lesion by intrarenal injection of phenol chronically increases central sympathetic activation resulting in hypertension, without affecting kidney function, in rats. ${ }^{(2)}$ The BP-elevation after the intrarenal injection can be prevented by prior denervation of the phenol injected kidney ${ }^{(2)}$, which demonstrates a crucial role of intact renal innervation for afferent signaling from the kidney to the vasomotor center.

Renal denervation (RDN), a catheter-based approach developed to disrupt the renal sympathetic nerves using radio frequency energy, is a promising therapy for resistant hypertension. The first clinical studies, in a relatively small number of patients, showed that this technique appears safe and effective. ${ }^{(3-5)}$ Office systolic blood pressure (SBP)/ diastolic blood pressure (DBP) reduced by 32/12 $\mathrm{mmHg}$ six months after RDN.(4) RDN is assumed to decrease sympathetic activity, resulting in altered sodium handling by the kidneys and a decrease in peripheral vascular resistance. Consequently, RDN may potentially improve renal oxygenation, and thereby remove the trigger for hypertension.

Blood oxygen level dependent (BOLD)-MRI provides a non-invasive tool to determine renal oxygenation in humans. BOLD-MRI uses the magnetic properties of deoxyhemoglobin. Deoxyhemoglobin is paramagnetic, causing a decrease in signal on $T_{2}{ }^{*}$ weighted images. The apparent relaxation rate $R_{2}^{*}\left(=1 / T_{2}^{*}\right)$ is proportional to the concentration of deoxyhemoglobin. Increased $\mathrm{R}_{2}{ }^{*}$ implies increased levels of deoxyhemoglobin which can be related to reduced partial pressures of oxygen in tissue. ${ }^{(6)}$ Renal oxygenation reflects the balance between regional oxygen-supply and -consumption, principally for tubular transport. ${ }^{(7)}$ There is a non-homogenous distribution of oxygen within the kidneys. $\mathrm{pO}_{2}$ sharply declines at the corticomedullary junction. The renal medulla functions at levels of limited oxygen supply, with a $\mathrm{pO}_{2}$ as low as $25 \mathrm{~mm} \mathrm{Hg}$ under normal physiologic conditions. ${ }^{(7)}$

The aim of current study was to investigate the effect of $\mathrm{RDN}$ on renal oxygenation determined by BOLD-MRI. 


\section{Methods}

\section{Study Population}

Both patients with resistant hypertension (defined as a SBP $\geq 160 \mathrm{~mm} \mathrm{Hg}$, despite use of $\geq 3$ antihypertensive drugs) as well as patients fulfilling the same BP criteria $(n=46)$, but without optimal pharmacological treatment due to intolerance for antihypertensive drugs were included in the present study $(n=8)$. Major contraindications for inclusion were: an estimated glomerular filtration rate (eGFR) $<30 \mathrm{~mL} / \mathrm{min} / 1.73 \mathrm{~m}^{2}$, secondary causes of hypertension, and a history of renal artery stenting or severe co-morbidity. All patients were screened using a standardized screening protocol, described in detail in a recent paper. ${ }^{(8)}$ The protocol had three aims: firstly to confirm the presence of hypertension using ambulatory BP monitoring (ABPM), secondly to exclude secondary forms of hypertension and finally to study the eligibility of the renal arteries to undergo RDN by contrast-enhanced magnetic resonance angiography (MRA).

The study protocol was carried out with the approval of the Ethics Committee of the University Medical Centre Utrecht, and all patients gave written informed consent. The current study is registered as NCTo1427049.

\section{Temporal Medication Stop}

At baseline and 12 months after RDN, we considered whether it would be safe to stop antihypertensive medication for two weeks prior to the tests (Appendix 1). ${ }^{(8)}$ This was done as part of our standardized clinical work-up for patients with complicated hypertension $^{(8)}$ to improve standardization of the test conditions since antihypertensive drugs influence plasma aldosterone concentration (PAC), plasma renin activity (PRA) and BOLD-MRI. If considered unsafe to stop antihypertensive medication, patients were switched to doxazosin and/or diltiazem, neutral drugs that do not influence eGFR, the RAAS and urine catecholamine levels.(9,10) Temporary wash out of interfering drugs is advised by the European Society of Hypertension and the Endocrine Society in the work-up for primary hyperaldosteronism. ${ }^{(9,11)}$ The decision to stop antihypertensive drugs was based on clinical judgement with emphasis on (cardiovascular) medical history. During the medication free interval, patients were regularly contacted by telephone by trained nurses or a physician. Also, patients were informed to contact us if they developed symptoms. Patients in whom it was considered unsafe to stop antihypertensive drugs or to use the neutral drugs mentioned above, were excluded from the present analysis. After the test period, patients restarted their medication. 


\section{Measurements}

All measurements at baseline, as part of the standardized screening protocol, and 12 months after RDN were performed during the medication free interval. An ABPM was taken non-invasively using WatchBP O3 (Microlife Inc., Widnau, Switzerland), with readings taken every $30 \mathrm{~min}$ during day and every $60 \mathrm{~min}$ at night. 24-hr urine was collected and sodium (mmol/24-hr) was analysed. Three days before the investigations, patients were asked to maintain a constant diet in order to avoid large fluctuations in sodium balance. A blood sample was drawn and creatinine $(\mu \mathrm{mol} / \mathrm{L})$ was determined.

\section{BOLD-MRI}

MRI's were performed in the afternoon. Patients were scanned either on a 1.5-T (Ingenia, software release 4.1, Philips Healthcare, Eindhoven, The Netherlands) or 3.0-T (Achieva, release 3.2, Philips Healthcare, Eindhoven, The Netherlands) commercially available MR systems. In brief, the MR imaging protocol consisted of obtaining localizer images to locate the kidneys, after which coronal (3 partitions) and transverse ( 6 partitions) $\mathrm{T}_{2}{ }^{*}$-weighted imaging was performed with the following echo times: $4.6 \mathrm{~ms}, 9.2 \mathrm{~ms}, 13.8 \mathrm{~ms}, 18.4 \mathrm{~ms}, 23 \mathrm{~ms}, 27.6 \mathrm{~ms}, 32.2 \mathrm{~ms}, 36.8 \mathrm{~ms}, 41.4 \mathrm{~ms}$, $46 \mathrm{~ms}$, $50.6 \mathrm{~ms}, 55.2 \mathrm{~ms}, 59.8 \mathrm{~ms}, 64.4 \mathrm{~ms}, 69 \mathrm{~ms}, 73.6 \mathrm{~ms}, 78.2 \mathrm{~ms}, 82.8 \mathrm{~ms}, 87,4 \mathrm{~ms}$, $92 \mathrm{~ms}$. The voxel sizes were $1.49 \times 1.50 \times 5.0 \mathrm{~mm}^{3}$ and the slice gap $7 \mathrm{~mm}$. Coronal field of view (FOV) was $375 \times 298 \mathrm{~mm}$ (1.5T, fold over direction (FD) right-left) or 298x375mm (3.oT, FD feet-head) and transversal FOV was 65×375mm (FD rightleft). Flip angle was $25^{\circ}$ and TR was $94 \mathrm{~ms}$ (1.5 T) or 95ms (3.0T). Regions of interest (ROI's) of the whole kidneys were drawn in the $\mathrm{T}_{2}{ }^{*}$ map. Areas affected by artifacts were avoided in the selection of the ROIs. The $\mathrm{R}_{2}{ }^{*}$ map was calculated from the $\mathrm{T}_{2}{ }^{*}$ data by fitting an exponential curve to individual voxels over the echo times. MRIs were analyzed using the compartmental method proposed by Ebrahimi et al.(12) using an in-house developed Matlab tool (MathWorks Inc, Natick, Massachusetts, USA). The histogram of the $\mathrm{R}_{2}{ }^{*}$ map was calculated and fitted to a Gaussian function (cortex) and a gamma function (medulla) (Figure 1). To objectively differentiate medulla, collecting system and vessels, a semi-automatic filter was applied to the $\mathrm{R}_{2}{ }^{*}$ maps. High intensity voxels, representing collecting system, and low intensity voxels, representing blood vessels, were excluded, as well as the four adjacent voxels (Figure 1). To obtain a measure for hypoxia, the cut-off $\mathrm{R}_{2}{ }^{*}$-value of the $10 \%$ most hypoxic voxels ( $90^{\text {th }}$ percentile of the histogram: P9o) was used (Figure 1). Muscle ROIs of the psoas muscle were obtained in the coronal slices in both the baseline and follow-up scan to assess scan reproducibility since muscle $\mathrm{R}_{2}^{*}$ is not affected by RDN. 


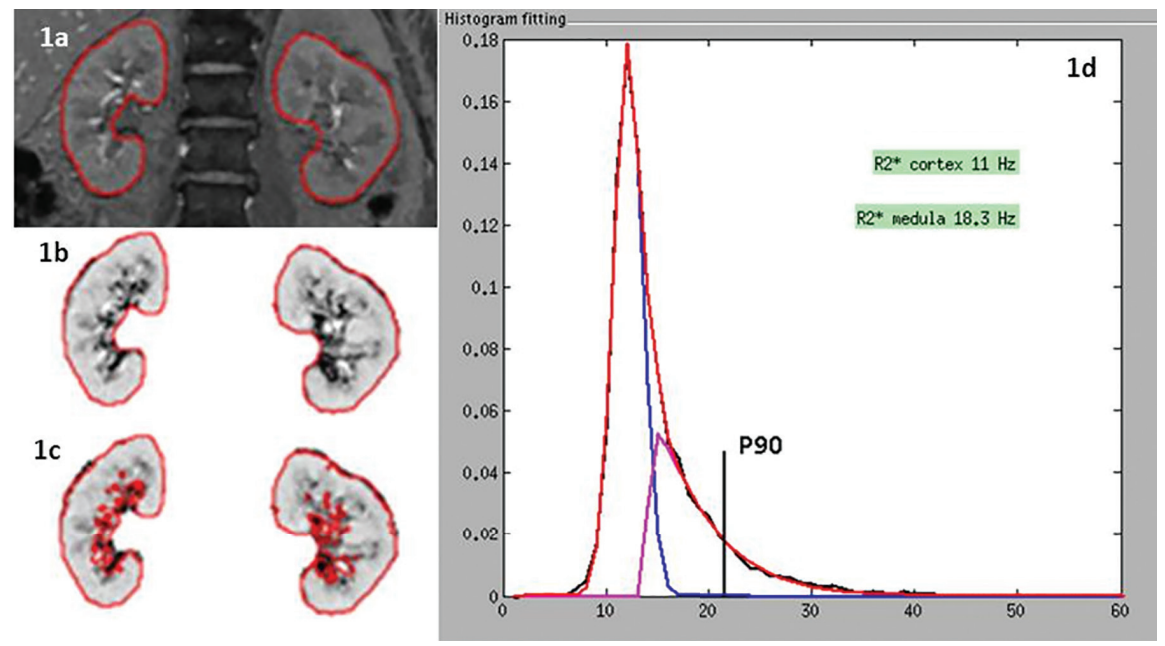

Figure 1. Analysis of BOLD MRI. ROI's are depicted in red; $\mathbf{a} \mathrm{T}_{2}{ }^{*}$ image of the kidneys; b $\mathrm{R}_{2}{ }^{*}$ image of the kidneys; c Exclusion of vessels and the collecting system. The collecting system is excluded by the filter (depicted in red); $\mathbf{d}$ histogram of the $\mathrm{R}_{2}^{*}$ map. According to the compartmental method, cortex is fitted to a Gaussian (blue) function and medulla to a gamma (pink) function. The sum (red) fits the histogram of the $\mathrm{R}_{2}{ }^{*}$ map shown in the Figure.

\section{Percutaneous renal denervation}

Renal angiograms were performed to confirm anatomic eligibility. 51 of 54 patients were treated using the Symplicity Flex device (Medtronic, Minneapolis, Minnesota). In these patients, using local anaesthetics, a 6Fr sheath was introduced via a femoral artery access site. Bilateral treatment of the arteries was performed using series of 2-minute radio frequency (RF) energy deliveries along each artery. ${ }^{(13)}$ These treatment points were made in a circumferential way with a minimum of $5 \mathrm{~mm}$ distance in between the treatment points. In 2 patients the EnligHTN system (St Jude, St. Paul, Minnesota) and in 1 patient the Oneshot system (Covidien, Mansfield, Massachusetts) was used. The EnligHTN system is a non-occlusive multi-electrode basket design, enabling 4 ablations in one session of 90 seconds. ${ }^{(14)}$ The Oneshot system is an irrigated RF balloon equipped with a spiral monopolar electrode for $360^{\circ}$ of ablation. ${ }^{(15)}$

\section{Data analysis}

A patient was classified as a responder of treatment with $\mathrm{RDN}$ when average daytime SBP determined by ABPM decreased by $\geq 5 \mathrm{~mm} \mathrm{Hg}$. Estimated glomerular filtration rate (eGFR) was calculated on the basis of the CKD-epi formula. ${ }^{(16)}$

Prescribed dosages of antihypertensive drugs were converted to defined daily doses (DDD) using conversion factors as provided by the World Health Organization (WHO) Drug Classification (http://www.whocc.no/atcddd/). Using DDD's and the total prescribed dosages, daily use (DU) of all antihypertensive drugs was calculated. 
The following subgroup analysis were performed: 1) patients who stopped medication twice regardless of the use of rescue medication (the latter do not influence BOLD-MRI), 2) patients who stopped medication twice and no rescue medication twice or used the same rescue medication. This approach was chosen since rescue medication does not influence BOLD-MRI and laboratory parameters, it does however influence BP.

Analysis of BOLD-MRI data was done by two investigators (EV and $\mathrm{AB}$ ), blinded to patient information and other results from the standardized screening protocol. Five patients from the $1.5 \mathrm{~T}$ scanner and five from the 3.0T scanner were randomly selected to determine intra-reader reproducibility. Eight patients from the 1.5 $\mathrm{T}$ scanner and eight patients from the 3.0T scanner were randomly selected to determine inter-reader reproducibility.

The number of voxels included in both the coronal and transverse slices was estimated to be around $8 \%$. It was chosen to use a weighted based on the number of voxels average $R_{2}{ }^{*}$-value of the coronal and transverse slices. To combine data from the $1.5 \mathrm{~T}$ and the 3.0T scanner, $\mathrm{z}$-scores for the change in $\mathrm{R}_{2}{ }^{*}$ levels for both field strengths were calculated and combined. A z-score is calculated for each individual as the obtained individual measurement minus the mean value of the group divided by the standard deviation around the group average ([Z score = (participant value $\mu) / \sigma])$.

\section{Statistical analysis}

All variables were reported as mean $\pm \mathrm{SD}$, median (range), or as proportion when appropriate. The Intra-class correlation coefficient (two-way mixed model, absolute agreement) and the Bland-Altman method (defining bias and variation by calculating the mean difference between the observers, the standard deviations of the differences and the $95 \%$ limits of agreement) were used to determine inter- and intrareader reproducibility.

The unpaired Students $t$-test and the chi-square test were used when applicable to compare baseline data between patients who stopped medication twice and the group who could not stop twice.

Paired sample analysis was done using the paired $t$-test or its nonparametric variant when applicable.

Linear regression analysis was used to analyze the association between the change in ABPM (independent variable) and change in $\mathrm{R}_{2}{ }^{*}$ in the cortex, medulla and P9o (dependent variables). Multivariable models were applied adjusting for variables related to BOLD and BP (change in eGFR, change in 24-hr urinary sodium excretion and the change in hemoglobin). The number of variables in the multivariable models was restricted by the limited sample size.

A two sided $P$ value of $<0.05$ was considered to be statistically significant. All 
analyses were performed with the SPSS statistical package version 20 (IBM SPSS Data Collection, Chicago, IL).

\section{Results}

Fifty-four patients were included in the present study, 50 patients completed followup. Baseline characteristics of the patients with a complete follow-up $(n=46)$, are shown in table 1. Thirty-two patients stopped medication twice of which 27 patients stopped medication twice and used the same or no rescue medication twice. Twentyseven patients (59\%) were scanned using a $1.5 \mathrm{~T}$ system, nineteen patients $(41 \%)$ with a $3 \mathrm{~T}$ system. Inter- and intrareader reproducibility of $\mathrm{R}_{2}^{*}$ values of the cortex, medulla and P90, were considered excellent based on the ICC (range: 0.957-0.999) as well as the Bland Altman method. (Appendix 2).

From 2 patients both baseline and follow-up scans (coronal and transverse scans) were excluded from analysis. From 2 patients both the coronal as well as the transverse scans made at baseline were excluded. From 42 patients pre- and post-imaging is available. In 7 of these patients only the coronal or transverse scan was excluded. Nine scans were excluded because of motion artifacts, 2 because of multiple cysts, 1 because of erroneous shimming and one because of multiple eddy current artifacts visible in the $\mathrm{R}_{2}{ }^{*}$-map.

Table 1. Patient characteristics.

\begin{tabular}{l|lll}
\hline & $\begin{array}{l}\text { Patients who } \\
\text { All patients } \\
\text { (N=46) }\end{array}$ & $\begin{array}{l}\text { Patients who did } \\
\text { twice (N=32) }\end{array}$ & $\begin{array}{l}\text { (Not stop medicati- } \\
\text { on twice (N=14) }\end{array}$ \\
\hline Age (yrs) & $57(11)$ & $58(11)$ & $57(11)$ \\
Sex (male/female) & $23 / 23$ & $18 / 14$ & $5 / 9$ \\
Comorbidity & & & \\
- Hypercholesterolemia & $27(59 \%)$ & $18(56 \%)$ & $9(64 \%)$ \\
- Diabetes Mellitus Type II & $8(17 \%)$ & $7(22 \%)$ & $1(8 \%)$ \\
- Cardiovascular diseases & $7(15 \%)$ & $4(13 \%)$ & $3(21 \%)$ \\
- Cerebral vascular diseases & $3(7 \%)$ & $1(3 \%)$ & $2(14 \%)$ \\
Office BP (mm Hg) under medication & $196(31) / 108(15)$ & $192(34) / 106(16)$ & $206(21) \dagger / 112(11)$ \\
Mean 24-hr BP (mm Hg)* & $163(20) / 98(14)$ & $169(16) / 101(12)$ & $150(22) \dagger / 93(15)$ \\
Mean day-time BP (mm Hg)* & $168(21) / 102(15)$ & $174(16) / 104(12)$ & $153(24) \dagger / 96(18)$ \\
Mean 24-hr HR during (bpm)* & $74(12)$ & $74(12)$ & $75(11)$ \\
Number of antihypertensive drugs & $4(0-8)$ & $4(0-8)$ & $5(2-6) \dagger$ \\
DU of antihypertensive drugs & $5.5(3.4)$ & $5.1(3.6)$ & $6.5(2.8)$ \\
Body-mass index (kg/m $\left.{ }^{2}\right)$ & $29.1(5.6)$ & $28.9(5 \cdot 7)$ & $29.5(5.6)$ \\
eGFR (mL/min/1.73m $\left.{ }^{2}\right)$ & $76(18)$ & $74(18)$ & $80(17)$
\end{tabular}

Continuous variables are displayed as a mean (SD), except for number of drugs, which is displayed as median (min-max). Categorical variables are displayed as a number (percentage). Yrs indicates: years, BP: blood pressure, HR: heart rate, DU: daily use, eGFR: estimated glomerular filtration rate; *Determined using ABPM; †Significant difference between the group of patients stopped medication twice and the group of patients who did not stop twice. 


\section{Renal denervation}

On average, $11.3( \pm 3.8)$ ablations were applied per patient. Periprocedurally, two patients had a minor bleeding at the puncture site, which was treated with compression.

\section{Change in blood pressure and kidney function after RDN.}

Mean 24-hr BP changed from 163( \pm 20$) / 98( \pm 14) \mathrm{mmHg}$ to $154( \pm 22) / 92( \pm 13) \mathrm{mmHg}$ $(\mathrm{p}=0.001$ and $\mathrm{p}<0.001$, table 2$)$. Mean daytime BP changed from $168( \pm 21) / 102( \pm 15)$ $\mathrm{mmHg}$ to $158( \pm 23) / 96( \pm 13) \mathrm{mmHg}(\mathrm{p}=0.001$ and $\mathrm{p}<0.001$, table 2$)$. The decrease is less pronounced in the subgroup of patients who stopped medication twice (twice no rescue medication or twice the same rescue medication), but still significant (table 2). Eleven patients (42\%) were classified as responder in this subgroup. Daytime heart rate (HR) did not change in the total group of patients and in the subgroup. The daily use of antihypertensive drugs did not change significantly $(5.5( \pm 3.4)$ vs $4.8( \pm 3.5) \mathrm{p}=0.18$, table 2$)$. eGFR remained stable 12 months after RDN compared to baseline $\left(77( \pm 18) \mathrm{mL} / \mathrm{min} / 1.73 \mathrm{~m}^{2}\right.$ vs $79( \pm 20) \mathrm{mL} / \mathrm{min} / 1.73 \mathrm{~m}^{2} \mathrm{p}=0.13$, table 2$)$.

Table 2. Baseline and follow-up data.

\begin{tabular}{|c|c|c|c|c|}
\hline & \multicolumn{2}{|c|}{ All patients $(n=46)$} & \multicolumn{2}{|c|}{$\begin{array}{l}\text { Patients who stopped } \\
\text { medication twice or had same } \\
\text { rescue medication }(n=27)\end{array}$} \\
\hline & Baseline & 12 months & Baseline & 12 months \\
\hline Mean 24-hr BP (mm Hg)* & $163(20) / 90(13)$ & $154(22)+\dagger / 92(13)^{\dagger \dagger}$ & $169(16) / 101(11)$ & $162(19) \dagger / 96(12) \dagger$ \\
\hline Mean day-time BP (mm Hg)* & 168(21)/102(15) & $\left.158(23)^{\dagger+/ 96(13)}\right)^{\dagger \dagger}$ & $174(16) / 105(11)$ & $167(20) \dagger / 100(11)^{\dagger}$ \\
\hline Mean 24-hr HR (bpm)* & $74(13)$ & $72(10)$ & $72(10)$ & $72(10)$ \\
\hline DU of antihypertensive drugs & $5.5(3.4)$ & $4.8(3.5)$ & $5 \cdot 3(3.8)$ & $4.3(3.8)$ \\
\hline eGFR (mL/min/1.73m²) & $77(18)$ & $79(20)$ & $77(18)$ & $82(19) \dagger$ \\
\hline
\end{tabular}

All variables are displayed as a mean (SD). HR indicates: heart rate, DU: daily use, eGFR: estimated glomerular filtration rate. *Determined using ABPM. ${ }^{\dagger} \mathrm{p}<0.05,{ }^{\dagger \dagger} \mathrm{p}<0.01$.

\section{Effect of RDN on renal oxygenation.}

As expected $\mathrm{R}_{2}^{*}{ }^{*}$ was higher on the 3.0-T MR. Table 3 a shows weighted mean baseline and 12-months follow up $\mathrm{R}_{2}^{*}$ values of the cortex, medulla and P9o for both field strengths. $\mathrm{R}_{2}^{*}$ did not change after $\mathrm{RDN}$, including in the subgroup of patients who stopped medication twice (table 3 ) or patients classified as responders (table 3 ). The change in $\mathrm{R}_{2}^{*}$ was not related to the change in day-time SBP in a univariable model, nor in multivariable models adjusting for the change in eGFR, the change in 24-hr sodium excretion and change in hemoglobin.

Muscle $\mathrm{R}_{2}^{*}$ did not change (table 3 ) implying good reproducibility. 
Table 3. Mean $\mathrm{R}_{2}^{*}$ before and after RDN.

\begin{tabular}{|c|c|c|c|c|}
\hline \multicolumn{5}{|c|}{ All patients $(n=41)$} \\
\hline & & R2*$^{*}$ baseline & R2* 12 m. follow-up & $\mathbf{p}$ \\
\hline \multirow[t]{4}{*}{$1.5 \mathrm{~T}$} & Cortex & $13.2(0.6)$ & $13.1(0.7)$ & 0.48 \\
\hline & Medulla & $20.5(0.9)$ & $19.9(1.2)$ & 0.26 \\
\hline & P9o & $21.2(1.4)$ & $20.6(1.9)$ & 0.25 \\
\hline & Muscle & $35.8(1.7)$ & $35.2(1.7)$ & 0.44 \\
\hline \multirow[t]{4}{*}{$3.0 \mathrm{~T}$} & Cortex & $17.3(0.2)$ & $17.7(0.8)$ & 0.45 \\
\hline & Medulla & $27.0(2.9)$ & $27.2(4.1)$ & 0.91 \\
\hline & P9o & $31.2(4.1)$ & $32.1(3 \cdot 3)$ & 0.55 \\
\hline & Muscle & $38.4(1.5)$ & $38.2(0.2)$ & 0.66 \\
\hline \multicolumn{5}{|c|}{ Patients who stopped medication twice $(n=27)$} \\
\hline \multirow[t]{4}{*}{$1.5 T$} & Cortex & $12.6(0.9)$ & $12.6(0.9)$ & 0.74 \\
\hline & Medulla & $19.7(0.9)$ & $19.3(1.4)$ & 0.32 \\
\hline & P9o & $20.6(1.6)$ & $20.1(1.9)$ & 0.19 \\
\hline & Muscle & $36.0(1.6)$ & $35 \cdot 7(1.7)$ & 0.41 \\
\hline \multirow[t]{4}{*}{$3.0 \mathrm{~T}$} & Cortex & $17.6(0.5)$ & $17.7(0.9)$ & 0.78 \\
\hline & Medulla & $26.7(2.0)$ & $26.6(1.8)$ & 0.87 \\
\hline & P9o & $30.4(3.0)$ & $30.6(2.5)$ & 0.77 \\
\hline & Muscle & $37.7(2.2)$ & $37.1(2.4)$ & 0.58 \\
\hline \multicolumn{5}{|c|}{ Patients who classified as a responder and stopped medication twice $(n=11)$} \\
\hline \multirow[t]{4}{*}{$1.5 \mathrm{~T}$} & Cortex & $12.5(0.9)$ & $12.5(0.9)$ & 0.94 \\
\hline & Medulla & $19.6(1.7)$ & $19.3(1.4)$ & 0.40 \\
\hline & P9o & $20.5(1.6)$ & $19.9(1.8)$ & 0.14 \\
\hline & Muscle & $35.8(1.6)$ & $35.4(1.9)$ & 0.23 \\
\hline \multirow[t]{4}{*}{$3.0 \mathrm{~T}$} & Cortex & $18.1(0.8)$ & $17.8(1.2)$ & 0.47 \\
\hline & Medulla & $27.4(1.9)$ & $26.7(1.8)$ & 0.19 \\
\hline & P9o & $31.3(2.8)$ & $30.6(2.4)$ & 0.26 \\
\hline & Muscle & $38.0(1.9)$ & $37 \cdot 3(2.6)$ & 0.29 \\
\hline
\end{tabular}

All variables are displayed as a mean (SD). $\mathrm{R}_{2}^{*}$ is expressed in $\mathrm{s}^{-1}$.

\section{Discussion}

In this study we demonstrate in hypertensive patients that renal BOLD did not change after treatment with RDN. Also in the subgroup of patients classified as responder, BOLD not did alter after treatment.

BOLD-MRI gives information on renal oxygenation. The oxygen level within the kidney reflects the balance between regional oxygen supply and oxygen consumption. Oxygen supply is controlled by systemic and intrarenal factors that 
control intrarenal blood-flow and distribution, oxygen capacity of the blood and the specific structure of the microcirculation of the kidney. The consumption is influenced by GFR, tubular and ion-pump mass and various regulators that control transport activity. ${ }^{(7)}$

Changes in either oxygen supply or demand alter the oxygen level. It is possible that RDN increases renal oxygenation as a consequence of decreased sympathetic activation resulting in a decreased renal vascular resistance and reduced activity of the RAAS. Decreased levels of aldosterone will result in reduced reabsorption of sodium in the collecting tubules, an active process. As a consequence of this, less oxygen will be used.

Our study shows that renal BOLD did not change after treatment with RDN although a reduction in BP was observed. This suggests that RDN did not affect renal oxygenation. Ott et al. showed that despite a decrease in BP, renal perfusion, determined by MRI with arterial spin labeling (ASL), did not change 3 months after $\mathrm{RDN}$ in 19 patients with resistant hypertension. Renal vascular resistance, calculated from BP and perfusion measured by ASL, was significantly reduced after RDN.(17) This is in line with another study showing that RDN reduced the renal resistive index, assessed by duplex ultrasonography, 6 months after RDN in 88 patients with resistant hypertension. ${ }^{(18)}$ So, it is very well possible that because renal perfusion did not change after RDN, also renal BOLD was unaffected. This can be the result of tight autoregulation in the kidneys.

Several issues need to be briefly discussed when considering the possible reason(s) for the absence of an effect, including both methodological, i.e. MRI related issues, but also intervention, i.e. $\mathrm{RDN}$ related issues.

It is important to realize that BOLD-MRI is sensitive to changes in medullary oxygenation since the medullary oxygen level lies on the linear part of the hemoglobin oxygenation curve, and is therefore sensitive to small changes in oxygen tension. ${ }^{(19)}$ BOLD-MRI is however less sensitive to changes in cortical oxygenation, because cortical blood $\mathrm{pO}_{2}$ lies on the shoulder of the curve. ${ }^{(19)}$ So, larger differences in oxygenation are necessary to observe similar changes in $\mathrm{R}_{2}^{*}$ for the cortex compared with the medulla. A possible small effect of RDN on cortical oxygenation could therefore be missed using BOLD-MRI.

P9o $\left(90^{\text {th }}\right.$ percentile of the histogram) gives information on the amount of hypoxia. This measure can be informative since in case of renal injury, hyperfiltration and hypertrophy of uninjured nephrons can take place. These nephrons have a high metabolic demand, in contrast with injured nephrons. As a consequence there can be a heterogeneous distribution of hypoxia in the kidney, which might not be detected using average $\mathrm{R}_{2}$ * . Our study shows that P9o also did not change after RDN.

Another possible reason for the absence of an effect is that the RDN procedure was unsuccessful, at least in some patients. The high percentage of non-responders 
in our study may indicate that $\mathrm{RDN}$ was not complete in these patients, and that as a consequence RDN did not affect BP and renal oxygenation. However in the subgroup of patients classified as responder, renal oxygenation did also not change.

We quantified the BP-lowering effect using ABPM. An ABPM offers a large number of BP measurements, during both day- and night-time. This results in a more precise assessment of BP compared to single measurements. ${ }^{(20)} \mathrm{ABPM}$ is also recommended in the follow-up of resistant hypertension in the guidelines from the European Society of Hypertension and the European Society of Cardiology. ${ }^{(11)}$ Our study shows that RDN significantly reduces BP, determined by ABPM. Fortytwo percent of the patients was classified as a responder. This percentage is lower compared to the study of Mahfoud et al investigating the effect of RDN on ABPM in 346 patients with uncontrolled hypertension (303 patients with resistant hypertension and 43 with pseudoresistant hypertension) 3,6 and 12 months after RDN. ${ }^{(21)}$ This studied identified $70 \%$ of the 80 patients with resistant hypertension with completed 12 months follow-up as responder (defined as a decrease in SBP $\geq 5 \mathrm{~mm} \mathrm{Hg}$ of the 24-hr ABPM). ${ }^{(21)}$

An important strength of our study is the standardization of antihypertensive drugs and the use of ABPM. This is important for quantifying the effects on both BP and BOLD-MRI. ABPM was assessed during a medication free interval before and 12 months after RDN. This excludes the potential disturbance by antihypertensive medication. Pharmacologic treatment is likely to be changed during the 12 months after RDN and possibly drug adherence can be improved in some patients. Other studies may have overestimated the effect of RDN due to confounding by pharmacological treatment. Further, some medications have been shown to affect renal BOLD. Several studies have shown the effect of furosemide on BOLD. ${ }^{(22-24)}$ This diuretic reduces the active sodium transport in the ascending loop of Henle, an oxygen consuming process. BOLD-MRI detect this drop in medullary and in a lesser degree cortical oxygenation. Moreover losartan, an angiotensin II receptor

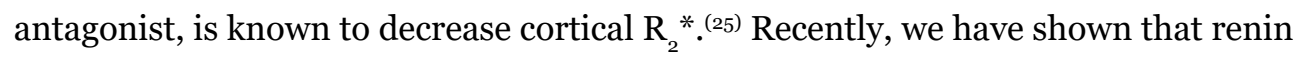
inhibitors affect the BOLD-signal. ${ }^{(26)}$ Unfortunately the medication stop was not possible in all patients.

Several limitations need to be mentioned. Assessment of oxygenation of the kidneys, either by direct measurement or indirectly by BOLD-MRI, does not distinguish between (changes in) oxygen supply and consumption. It is therefore not possible to investigate the association between renal blood flow or oxygen consumption (mostly influenced by tubular transport) and the variables investigated in the present study. Further, a specific limitation of our study can be the use of two different field strengths. To combine the data, we used z-scores.

In conclusion, our study shows in patients with hypertension that RDN does not alter renal oxygenation as assessed by BOLD-MRI. 


\section{References}

1. Welch WJ. Intrarenal oxygen and hypertension. Clin Exp Pharmacol Physiol. 2006;33(10):1002-5.

2. Ye S, Gamburd M, Mozayeni P, Koss M, Campese VM. A limited renal injury may cause a permanent form of neurogenic hypertension. Am J Hypertens. 1998;11(6 Pt 1):723-8.

3. Krum H, Schlaich M, Whitbourn R, Sobotka PA, Sadowski J, Bartus K, et al. Catheterbased renal sympathetic denervation for resistant hypertension: a multicentre safety and proof-of-principle cohort study. Lancet. 2009;373(9671):1275-81.

4. Symplicity HTNI, Esler MD, Krum H, Sobotka PA, Schlaich MP, Schmieder RE, et al. Renal sympathetic denervation in patients with treatment-resistant hypertension (The Symplicity HTN-2 Trial): a randomised controlled trial. Lancet. 2010;376(9756):19039.

5. Symplicity HTNI. Catheter-based renal sympathetic denervation for resistant hypertension: durability of blood pressure reduction out to 24 months. Hypertension. 2011;57(5):911-7.

6. Li LP, Halter S, Prasad PV. Blood oxygen level-dependent MR imaging of the kidneys. Magn Reson Imaging Clin N Am. 2008;16(4):613-25, viii.

7. Heyman SN, Khamaisi M, Rosen S, Rosenberger C. Renal parenchymal hypoxia, hypoxia response and the progression of chronic kidney disease. Am J Nephrol. 2008;28(6):998-1006.

8. Verloop WL, Vink EE, Voskuil M, Vonken EJ, Rookmaaker MB, Bots ML, et al. Eligibility for percutaneous renal denervation: the importance of a systematic screening. J Hypertens. 2013;31(8):1662-8.

9. Funder JW, Carey RM, Fardella C, Gomez-Sanchez CE, Mantero F, Stowasser M, et al. Case detection, diagnosis, and treatment of patients with primary aldosteronism: an endocrine society clinical practice guideline. $J$ Clin Endocrinol Metab. 2008;93(9):3266-81.

10. Shionoiri H, Yasuda G, Yoshimura H, Umemura S, Miyajima E, Miyakawa T, et al. Antihypertensive effects and pharmacokinetics of single and consecutive administration of doxazosin in patients with mild to moderate essential hypertension. J Cardiovasc Pharmacol. 1987;10(1):90-5.

11. Mancia G, Fagard R, Narkiewicz K, Redon J, Zanchetti A, Bohm M, et al. 2013 ESH/ ESC Guidelines for the management of arterial hypertension: the Task Force for the management of arterial hypertension of the European Society of Hypertension (ESH) and of the European Society of Cardiology (ESC). J Hypertens. 2013;31(7):1281-357.

12. Ebrahimi B, Gloviczki M, Woollard JR, Crane JA, Textor SC, Lerman LO. Compartmental analysis of renal BOLD MRI data: introduction and validation. Invest Radiol. 2012;47(3):175-82.

13. Schlaich MP, Sobotka PA, Krum H, Lambert E, Esler MD. Renal sympathetic-nerve 
ablation for uncontrolled hypertension. N Engl J Med. 2009;361(9):932-4.

14. Worthley SG, Tsioufis CP, Worthley MI, Sinhal A, Chew DP, Meredith IT, et al. Safety and efficacy of a multi-electrode renal sympathetic denervation system in resistant hypertension: the EnligHTN I trial. Eur Heart J. 2013;34(28):2132-40.

15. Ormiston JA, Watson T, van Pelt N, Stewart R, Haworth P, Stewart JT, et al. First-inhuman use of the OneShot renal denervation system from Covidien. EuroIntervention. 2013;8(9):1090-4.

16. Levey AS, Stevens LA, Schmid CH, Zhang YL, Castro AF, 3rd, Feldman HI, et al. A new equation to estimate glomerular filtration rate. Ann Intern Med. 2009;150(9):604-12.

17. Ott C, Janka R, Schmid A, Titze S, Ditting T, Sobotka PA, et al. Vascular and renal hemodynamic changes after renal denervation. Clin J Am Soc Nephrol. 2013;8(7):1195201.

18. Mahfoud F, Cremers B, Janker J, Link B, Vonend O, Ukena C, et al. Renal hemodynamics and renal function after catheter-based renal sympathetic denervation in patients with resistant hypertension. Hypertension. 2012;60(2):419-24.

19. Prasad PV. Evaluation of intra-renal oxygenation by BOLD MRI. Nephron Clin Pract. 2006;103(2):c58-65.

20. Waeber B, Brunner HR. Clinical value of ambulatory blood pressure monitoring in the assessment of antihypertensive therapy. Blood Press Monit. 1999;4(5):263-6.

21. Mahfoud F, Ukena C, Schmieder RE, Cremers B, Rump LC, Vonend O, et al. Ambulatory blood pressure changes after renal sympathetic denervation in patients with resistant hypertension. Circulation. 2013;128(2):132-40.

22. Gloviczki ML, Glockner JF, Lerman LO, McKusick MA, Misra S, Grande JP, et al. Preserved oxygenation despite reduced blood flow in poststenotic kidneys in human atherosclerotic renal artery stenosis. Hypertension. 2010;55(4):961-6.

23. Textor SC, Glockner JF, Lerman LO, Misra S, McKusick MA, Riederer SJ, et al. The use of magnetic resonance to evaluate tissue oxygenation in renal artery stenosis. J Am Soc Nephrol. 2008;19(4):780-8.

24. Epstein FH, Prasad P. Effects of furosemide on medullary oxygenation in younger and older subjects. Kidney Int. 2000;57(5):2080-3.

25. Djamali A, Sadowski EA, Muehrer RJ, Reese S, Smavatkul C, Vidyasagar A, et al. BOLD-MRI assessment of intrarenal oxygenation and oxidative stress in patients with chronic kidney allograft dysfunction. Am J Physiol Renal Physiol. 2007;292(2):F51322.

26. Siddiqi L, Hoogduin H, Visser F, Leiner T, Mali WP, Blankestijn PJ. Inhibition of the renin-angiotensin system affects kidney tissue oxygenation evaluated by magnetic resonance imaging in patients with chronic kidney disease. $J$ Clin Hypertens (Greenwich). 2014;16(3):214-8. 


\section{Supplementary Methods - temporary medication stop}

Table 1. Scheme of gradual discontinuation of medication.

\begin{tabular}{|c|c|c|}
\hline \multirow[t]{8}{*}{4 weeks before measurements } & \multicolumn{2}{|c|}{$\begin{array}{l}\text { Stop: diuretics (including aldactone) and aliskiren } \\
\text { Gradually reduced: } \beta \text {-blockers and central working antihypertensive } \\
\text { drugs are reduced in two weeks: }\end{array}$} \\
\hline & Day 1: $100 \%$ & Day 8: o\% \\
\hline & Day 2: $50 \%$ & Day 9: $25 \%$ \\
\hline & Day 3: 50\% & Day 10: 0\% \\
\hline & Day 4: 50\% & Day 11: $25 \%$ \\
\hline & Day 5: 50\% & Day 12: o\% \\
\hline & Day 6: o\% & Day $13: 25 \%$ \\
\hline & Day 7: 50\% & Day 14: 0\% \\
\hline 2 weeks before measurements & \multicolumn{2}{|c|}{$\begin{array}{l}\text { Stop: ACE-inhibitors, AT1-antagonists, Calcium-antagonists, } \alpha \text {-blockers, } \\
\text { direct vasodilatators }\end{array}$} \\
\hline
\end{tabular}

\section{Supplementary Results - reproducibility of $\mathbf{R}_{2}^{*}$ measurements}

Table S1. Inter- and intrareader reproducibility of $\mathrm{R}_{2}^{*}$ measurements.

\begin{tabular}{ll|ll}
\hline & & ICC & \\
\hline \multirow{2}{*}{ Transverse } & Cortex & 0.995 & 95\%CI \\
& Medulla & 0.932 & $0.987-0.998$ \\
\hline Coronal & Cortex & 0.999 & $0.926-0.975$ \\
\hline & Medulla & 0.980 & $0.947-0.993$ \\
\hline Transverse & Cortex & Intrareader reproducibility - EV & \\
& Medulla & 0.999 & $0.993-1.000$ \\
\hline Coronal & Cortex & 0.957 & $0.538-0.991$ \\
& Medulla & 0.996 & $0.986-0.999$ \\
& & 0.992 & $0.966-0.998$ \\
\hline Transverse & Cortex & Intrareader reproducibility - AB & \\
& Medulla & 0.998 & $0.993-1.000$ \\
& Cortex & 0.989 & $0.935-0.997$ \\
\hline Coronal & Medulla & 0.999 & $0.998-1.000$ \\
& & 0.986 & $0.945-0.997$ \\
\hline
\end{tabular}

ICC: Intra-class correlation coefficient (two-way mixed model, absolute agreement). 95\% CI: 95\% confidence interval, SD: standard deviation. 

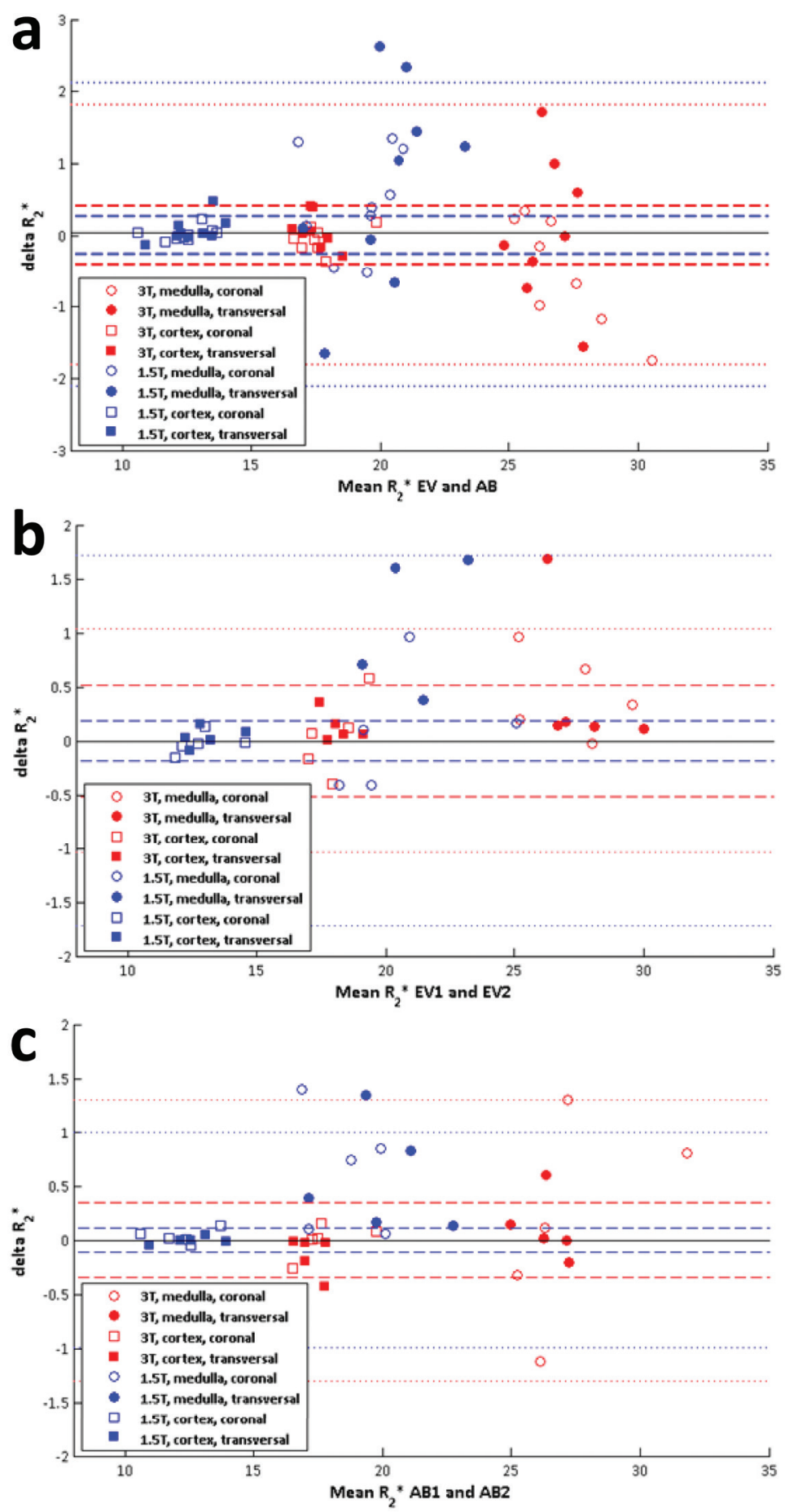

Figure S1. Bland Altman plots for a interreader reproducibility; b intrareader reproducibility of reader $\mathrm{EV}$; $\mathbf{c}$ intrareader reproducibility reader $\mathrm{AB}$ of $\mathrm{R}_{2}{ }^{*}$ measurements. 



\section{Chapter 6}

\section{Renal BOLD MRI Relates to Kidney Function and}

Activity of the Renin-Angiotensin-Aldosterone System in Hypertensive Patients

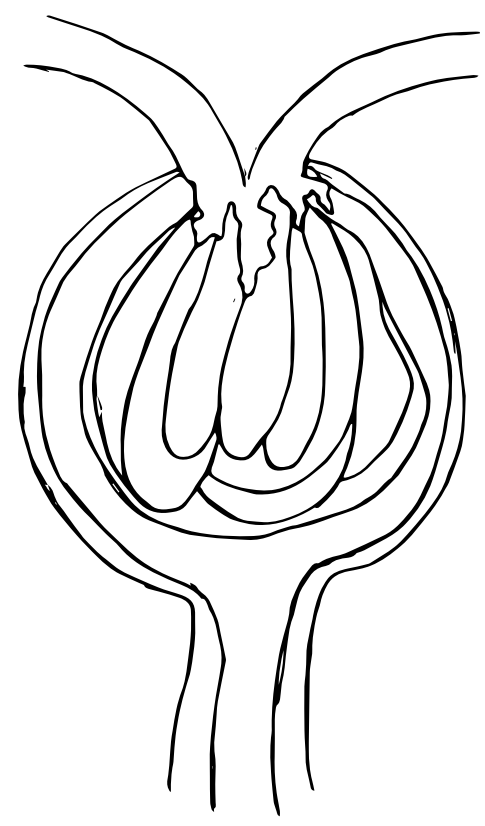

\section{Author contributions}

Vink EE, de Boer A, Hoogduin JM, Voskuil M, Leiner T, Bots ML, Joles JA, Blankestijn PJ Published in

J Hypertens 2015; doi:10.1097/HJH.0000000000000436 


\section{Abstract}

Background The renin angiotensin aldosterone system (RAAS) and the sympathetic nervous system (SNS) are key factors in the pathophysiology of hypertension. Renal hypoxia is the putative mechanism stimulating both systems. Blood oxygen level dependent MRI (BOLD MRI) provides a non-invasive tool to determine renal oxygenation in humans. The aim of the current study was to investigate the relation between kidney function as well as direct and indirect variables of the RAAS, and SNS with renal BOLD MRI. This information is crucial in the interpretation of renal BOLD MRI.

Design 75 hypertensive patients (38 males) were included. Antihypertensive medication was temporarily stopped. Patients collected urine during 24-hr (sodium, creatinine, catecholamines), blood samples were taken (creatinine, renin, aldosterone), a captopril challenge test was performed and 24-hr ambulatory BP was taken. Imaging data were performed on a 1.5-T (55 patients) or on a 3.0-T system (20 patients).

Results Mean age was $58( \pm 11)$ years, day-time BP was $167( \pm 19) / 102( \pm 16)$ $\mathrm{mmHg}$, eGFR was $75( \pm 18) \mathrm{mL} / \mathrm{min} / 1.73 \mathrm{~m}^{2}$. In multivariable regression analysis, renal medullary $\mathrm{R}_{2}{ }^{*}$ inversely related to eGFR $(\mathrm{p}=0.02)$. A non-dipping pattern of $\mathrm{BP}$, a variable related to increased sympathetic activity, was positively related to medullary $\mathrm{R}_{2}{ }^{*}(\mathrm{p}=0.02)$ and to P90, a measure for the amount of hypoxia, $(\mathrm{p}=0.02)$ in a multivariable analysis. Moreover the BP-lowering effect of captopril positively related to cortical- $(\mathrm{p}=0.02)$ and medullary $(\mathrm{p}=0.008) \mathrm{R}_{2}{ }^{*}$ as well as to $\mathrm{P} 9 \mathrm{O}(\mathrm{p}=0.02)$.

Conclusion In patients with hypertension, kidney function as well as direct and indirect variables of the RAAS and SNS relate to renal $\mathrm{R}_{2}{ }^{*}$, which can be interpreted as a measure of renal oxygenation. 


\section{Introduction}

The kidneys are known to play a crucial role in the pathophysiology of hypertension. Animal studies found that renal ischemia may be a central event in this pathogenesis. Renal ischemia is the putative common mechanism stimulating both the renin angiotensin aldosterone system (RAAS) and high sympathetic nerve activity.(1) Stimulation of renal sympathetic nerves results in a cascade of actions in the kidneys. As a result of renal vasoconstriction renal blood flow and glomerular filtration rate decrease, sodium reabsorption increases and Angiotensin II (AngII) is produced. AngII directly causes vasoconstriction, has trophic effects and plays an important role in water and sodium chloride reabsorption in the proximal tubule. ${ }^{(2)}$ These actions are thought to influence renal oxygenation. ${ }^{(3)}$

Blood oxygen level dependent (BOLD)-MRI provides a non-invasive tool to determine renal oxygenation in humans. BOLD-MRI uses the magnetic properties of deoxyhemoglobin. Deoxyhemoglobin is paramagnetic, causing a decrease in signal on $T_{2}{ }^{*}$ weighted images. The apparent relaxation rate $R_{2}^{*}\left(=1 / T_{2}^{*}\right)$ is proportional to the content of deoxyhemoglobin. Increased $\mathrm{R}_{2}^{*}$ implies increased levels of deoxyhemoglobin and decreased partial pressures of oxygen in tissue.(4) Renal oxygenation reflects the balance between regional oxygen-supply and -consumption, principally for tubular transport. ${ }^{(5)}$ There is a non-homogenous distribution of oxygen within the kidneys. $\mathrm{pO}_{2}$ sharply declines at the cortico-medullary junction. The renal medulla functions at levels of limited oxygen supply, with a $\mathrm{pO}_{2}$ as low as 25mmHg under normal physiologic conditions. ${ }^{(5)}$

Relations between kidney function as well as direct and indirect variables of the RAAS and SNS and renal BOLD MRI are unknown. Such information may help in the interpretation of renal BOLD MRI. Aim of present study is to investigate these relations in patients with hypertension.

\section{Methods}

\section{Study Population}

Patients with complicated hypertension referred to the University Medical Center Utrecht (European Society of Hypertension excellence center) for treatment with renal denervation were screened using a standardized protocol. ${ }^{(6)}$ The aims of this work-up were: to confirm the diagnosis of resistant hypertension, to exclude secondary causes of hypertension, and to obtain non-invasive imaging of the kidneys and renal arteries using MRI and MR angiography (MRA). BOLD MRI has been incorporated into this routine clinical protocol. Patients with a secondary cause of hypertension were excluded from the current analysis. 


\section{Temporary Medication Stop}

As part of the standardized screening protocol, all patients were discussed in our multidisciplinary team, where we considered whether it would be safe to stop antihypertensive medication for two weeks prior to the tests (Appendix 1). This was done as part of our standardized clinical work-up for patients with complicated hypertension(6) to improve standardization of the test conditions since antihypertensive drugs influence plasma aldosterone concentration (PAC), plasma renin activity (PRA) and BOLD MRI. If considered unsafe to stop antihypertensive medication, patients used doxazosin and/or diltiazem, neutral drugs that do not influence eGFR, the RAAS and urine catecholamine levels. ${ }^{(7,8)}$ Temporary wash out of interfering drugs is advised by the European Society of Hypertension and the Endocrine Society in the work-up for primary hyperaldosteronism. ${ }^{(7,9)}$ The decision to stop antihypertensive drugs was based on clinical judgement with emphasis on (cardiovascular) medical history. During the medication free interval, patients were regularly contacted by telephone by trained nurses or a physician. Also, patients were informed to contact us if they developed symptoms. Patients in whom it was considered unsafe to stop antihypertensive drugs or to use the neutral drugs mentioned above, were excluded from the present analysis. After the test period, patients restarted their medication.

\section{Measurements}

In all patients, we used data collected from the standardized work-up protocol for the present analysis. All patients visited the clinical research department to obtain blood samples for routine lab including creatinine $(\mu \mathrm{mol} / \mathrm{L})$, sodium $(\mathrm{mmol} / \mathrm{L})$ and haemoglobin ( $\mathrm{Hb} ; \mathrm{mmol} / \mathrm{L}$ ). The RAAS system was evaluated. Firstly, blood samples for determination of PAC (pmol/L) and PRA (fmol/L/s) were obtained after $90 \mathrm{~min}$ in supine and after $90 \mathrm{~min}$ in standing position. Subsequently, a captopril challenge test (CCT)(10) was performed while the patient was in supine position. After baseline BP assessments, $25 \mathrm{mg}$ of captopril was taken orally, where after BP was measured at $30,60,90$ and $120 \mathrm{~min}$.

One week before the scheduled visit to the clinical research unit, plasma potassium concentration was determined. In case of hypokalemia, supplementation was prescribed to prevent hypokalemia during measurements of PAC and PRA. Moreover, three days before the investigations, patients were asked to maintain a constant diet in order to avoid large fluctuations in sodium balance.

During the medication-free interval an ambulatory BP monitoring (ABPM) was done to confirm the diagnosis of hypertension. ABPM was taken non-invasively using WatchBP $\mathrm{O}_{3}$ (Microlife Inc., Widnau, Switzerland), with readings taken every 30 min during the day and every $60 \mathrm{~min}$ at night. Moreover urine was collected during24-hr to determine sodium ( $\mathrm{mmol} / 24 \mathrm{hr}$ ), creatinine ( $\mathrm{mmol} / 24 \mathrm{hr}$ ), albumin 
$(\mathrm{mg} / 24 \mathrm{hr})$, noradrenaline $(\mathrm{nmol} / 24 \mathrm{hr})$, dopamine $(\mathrm{nmol} / 24 \mathrm{hr})$, vanillylmandelic acid (VMA, $\mu \mathrm{mol} / 24 \mathrm{hr}$ ), metanephrine $(\mu \mathrm{mol} / 24 \mathrm{hr})$ and normetanephrine ( $\mu \mathrm{mol} / 24 \mathrm{hr})$.

\section{BOLD MRI}

MRI's were performed in the same week as the visit to the clinical research unit, in the afternoon. Patients were scanned either on a 1.5T (Ingenia, software release 4.1, Philips Healthcare, Eindhoven, The Netherlands) or 3.oT (Achieva Multitransmit, release 3.2, Philips Healthcare, Eindhoven, The Netherlands) commercially available MR systems. In brief, the MR imaging protocol consisted of obtaining localizer images to locate the kidneys, after which coronal (3 partitions) and transverse (6 partitions) $\mathrm{T}_{2}{ }^{*}$-weighted imaging was performed with 20 different echo times (echo time $4.6 \mathrm{~ms}$, delta echo time $4.6 \mathrm{~ms}$ ) with voxel size $1.49 \times 1.50 \times 5.0 \mathrm{~mm} 3$ and slice gap $7 \mathrm{~mm}$. Coronal field of view (FOV) was 375x298mm (1.5T, fold over direction (FD) right-left) or 298x375mm (3.0T, FD feet-head) and transversal FOV was $65 \times 375 \mathrm{~mm}$ (FD right-left). Flip angle was $25^{\circ}$ and TR was $94 \mathrm{~ms}(1.5 \mathrm{~T}$ ) or 95ms (3.oT). Regions of interest (ROI's) of the whole kidneys were placed in the $\mathrm{T}_{2}{ }^{*}$ map. Areas affected by artifacts were avoided in the selection of the ROIs. The $\mathrm{R}_{2}^{*}$ map was calculated from the $\mathrm{T}_{2}{ }^{*}$ weighted images by fitting an exponential curve to individual voxels over the echo times. MRIs were analyzed using the compartmental method proposed by

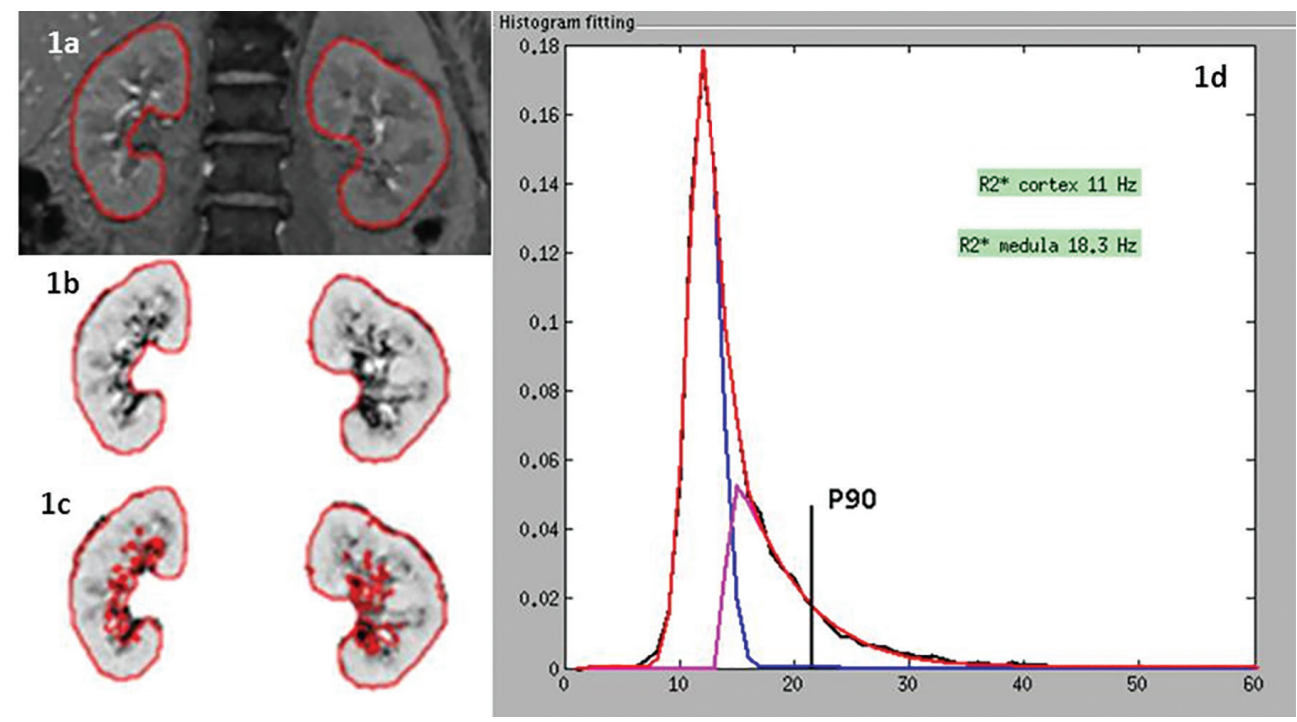

Figure 1. Analysis of BOLD. ROI's are depicted in red; $\mathbf{a} \mathrm{T}_{2}{ }^{*}$ weighted image of the kidneys; b $\mathrm{R}_{2}{ }^{*}$ image of the kidneys; $\mathbf{c}$ exclusion of vessels and the collecting system. The collecting system is excluded by the filter (depicted in red); $\mathbf{d}$ histogram of the $\mathrm{R}_{2}^{*}$ map; according to the compartmental method, cortex is fitted to a Gaussian (blue) function and medulla to a gamma (pink) function. The sum (red) fits the histogram of the $\mathrm{R}_{2}{ }^{*}$ map shown in the Figure. 
Table 1. Patient characteristics.

\begin{tabular}{l|cccc}
\hline & $\begin{array}{c}\text { All patients } \\
(\mathbf{N = 7 5 )}\end{array}$ & $\begin{array}{c}\text { Patients imaged } \\
\text { on 1.5T (N=55) }\end{array}$ & $\begin{array}{c}\text { Patients imaged } \\
\text { on 3.0T (N=20) }\end{array}$ & $\boldsymbol{p}^{\dagger}$ \\
\hline Age (yrs) & $58(11)$ & $58(11)$ & $59(10)$ & 0.74 \\
Sex (male/female) & $38 / 37$ & $27 / 28$ & $11 / 9$ & 0.65 \\
Comorbidity & & & $9(45 \%)$ & 0.15 \\
- Hypercholesterolemia & $44(60 \%)$ & $35(64 \%)$ & $6(30 \%)$ & 0.08 \\
- Diabetes Mellitus Type II & $11(15 \%)$ & $5(9 \%)$ & $5(25 \%)$ & 0.18 \\
- Cardiovascular diseases & $15(20 \%)$ & $10(18 \%)$ & $3(15 \%)$ & $0.26 / 0.06$ \\
$\begin{array}{l}\text { Office BP (mm Hg) } \\
\text { under medication }\end{array}$ & $6(8 \%)$ & $3(6 \%)$ & $196(29) / 108(16)$ & $0.45 / 0.87$ \\
$\begin{array}{l}\text { Mean day-time* BP } \\
\text { (mm Hg) }\end{array}$ & $189(32) / 103(15)$ & $186(33) / 101(13)$ & $170(22) / 102(13)$ & 0.83 \\
$\begin{array}{l}\text { Number of antihyper- } \\
\text { tensive drugs }\end{array}$ & $4(0-8)$ & $166(17) / 102(17)$ & $4(0-7)$ & 0.81 \\
$\begin{array}{l}\text { Body-mass index } \\
\text { (kg/m })\end{array}$ & $28.9(5.2)$ & $4(0-8)$ & $29.1(5.2)$ & 0.76 \\
eGFR (mL/min/1.73m $\left.{ }^{2}\right)$ & $75(18)$ & $28.8(5.2)$ & & $74(21)$ \\
\hline
\end{tabular}

Continuous variables are displayed as a mean (SD), except for number of drugs, which is displayed as median (range). Categorical variables are displayed as a number (percentage). Abbreviations: Yrs: years, BP: blood pressure, eGFR: estimated glomerular filtration rate; *Mean BP, determined using ambulatory BP monitoring; †Difference between patients scanned on 1.5 and 3.0T.

Table 2. Laboratory variables.

\begin{tabular}{|c|c|c|}
\hline Blood & $\mathbf{N}$ & \\
\hline $\mathrm{Hb}(\mathrm{mmol} / \mathrm{L})$ & 72 & $8.9(5.5-10.4)$ \\
\hline eGFR (mL/min/1.73m2) & 75 & $75(18)$ \\
\hline PAC - standing (pmol/L ) & 72 & $415(40-1870)$ \\
\hline PAC - supine (pmol/L) & 72 & $160(30-1220)$ \\
\hline PAC - after captopril (fmol/L/s) & 68 & $100(30-770)$ \\
\hline PRA - standing (fmol/L/s) & 72 & $225(48-3800)$ \\
\hline PRA - supine (fmol/L/s) & 72 & $150(40-2400)$ \\
\hline PRA - after captopril (fmol/L/s) & 69 & $160(40-8560)$ \\
\hline \multicolumn{3}{|l|}{ Urine ( 24-hr) } \\
\hline Sodium (mmol/ 24-hr) & 74 & $146.3(58.1)$ \\
\hline Albumin-creatinine ratio (mg/mmol) & 73 & $2.2(0.2-119.6)$ \\
\hline Adrenaline ( nmol/ 24-hr) & 62 & $31(4-126)$ \\
\hline Noradrenaline (nmol/ 24-hr) & 62 & $263(113)$ \\
\hline Dopamine & 61 & $1348(512)$ \\
\hline VMA ( $\mu \mathrm{mol} / \mathbf{2 4 - h r})$ & 59 & $22(8)$ \\
\hline Metanephrine ( $\mu \mathrm{mol} / \mathrm{24}-\mathrm{hr}$ ) & 74 & $0.7(0.1-2.4)$ \\
\hline Normetanephrines ( $\mu \mathrm{mol} / \mathrm{24}-\mathrm{hr}$ ) & 74 & $1.9(0.8-4.2)$ \\
\hline
\end{tabular}

Variables are displayed as a mean (SD) or median (range) when appropriate. Abbreviations: Hb: hemoglobin, eGFR: effective glomerular filtration rate, PAC: plasma aldosterone concentration, PRA: plasma renin activity, FENA: fractional sodium excretion, VMA: vanillylmandelic acid. 
Table 3. Mean $\mathrm{R}_{2}^{*}$ of scans included in the analysis.

\begin{tabular}{ll|ll}
\hline & & $\begin{array}{c}1.5 T(\mathbf{n}=51) \\
\mathbf{R}_{2}^{*}\left(\mathbf{s}^{-1}\right)\end{array}$ & $\begin{array}{c}\mathbf{3 . 0} \mathbf{T}(\mathbf{n}=\mathbf{1 9}) \\
\mathbf{R}_{2}^{*}\left(\mathbf{s}^{-1}\right)\end{array}$ \\
\hline Transverse & Cortex & $12.8(0.9)$ & $17.9(0.6)$ \\
& Medulla & $19.8(2.0)$ & $27.3(1.9)$ \\
& P9o & $20.9(2.2)$ & $29.8(3.0)$ \\
\hline Coronal & Cortex & $12.6(0.8)$ & $17.8(0.8)$ \\
& Medulla & $19.6(1.9)$ & $26.5(1.6)$ \\
\hline Weighted average & Poo & $20.1(2.5)$ & $29.8(3.0)$ \\
\hline & Cortex & $12.6(0.8)$ & $27.6(1.8)$ \\
& Medulla & $19.7(1.8)$ & $30.8(3.3)$ \\
\hline
\end{tabular}

Variables are displayed as a mean (SD).

Table 4. Mean $\mathrm{R}_{2}^{*}$ of scans included in the analysis.

\begin{tabular}{|c|c|c|c|c|c|c|}
\hline & \multicolumn{2}{|c|}{ Cortex } & \multicolumn{2}{|c|}{ Medulla } & \multicolumn{2}{|r|}{ P9o } \\
\hline & B & $95 \%$ CI & B & $95 \% \mathrm{CI}$ & B & $95 \% \mathrm{CI}$ \\
\hline $\begin{array}{l}\operatorname{eGFR}(\mathbf{m L} / \\
\left.\min / 1.73 \mathbf{m}^{2}\right)^{I I}\end{array}$ & -0.005 & $-0.02-0.01$ & -0.02 & $-0.03^{--0.003^{\dagger}}$ & -0.01 & $-0.03-0.003$ \\
\hline $\begin{array}{l}\text { Presence of a } \\
\text { non-dipping profile }{ }^{\mathrm{IV}}\end{array}$ & 0.23 & $-0.24-0.70$ & 0.57 & $0.11-1.04^{\dagger}$ & 0.60 & $0.11-1.10^{\dagger}$ \\
\hline $\begin{array}{l}\text { Noradrenaline } \\
\text { (nmol/ 24-hr) }^{\mathrm{I}}\end{array}$ & 0.002 & $-0.0004-0.004$ & 0.001 & $-0.001-0.004$ & $-0,0001$ & -0.003-0.002 \\
\hline $\begin{array}{l}\text { Noradrenaline } \\
\text { (nmol/ 24-hr) }^{\mathrm{III}}\end{array}$ & 0.001 & $-0.002-0.003$ & 0.0001 & $-0.002-0.002$ & -0.001 & -0.003-0.002 \\
\hline $\begin{array}{l}\text { Log-PAC - supine } \\
(\mathrm{pmol} / \mathrm{L})^{\mathrm{IV}}\end{array}$ & -0.11 & $-0.85-0.64$ & -0.656 & $-1.41-0.10$ & -0.55 & $-1.37-0.26$ \\
\hline $\begin{array}{l}\text { Log-PRA - supine } \\
(\mathrm{fmol} / \mathrm{L} / \mathrm{s})^{\mathrm{IV}}\end{array}$ & -0.54 & $-1.16-0.08$ & -0.09 & $-0.75^{-0.57}$ & -0.003 & $-0.71-0.71$ \\
\hline $\begin{array}{l}\text { Log- } \Delta \text { SBP }\left(\mathrm{mm}_{\mathrm{Hg}}\right) \\
\text { after captopril }\end{array}$ & -8.40 & $-15.35^{--1.44^{\dagger}}$ & -9.69 & $-16.80--2.58 \dagger$ & -9.18 & $-16.85^{--1.51^{\dagger}}$ \\
\hline
\end{tabular}

$\beta$ indicates regression coefficient: the change in outcome per one unit increase in exposure, $95 \% \mathrm{CI}$ : $95 \%$ confidence interval, N: number of patients; $\uparrow \mathrm{P}<0.05$; ${ }^{\text {I }}$ Model I: univariable model; II Model II: adjustment for BOLD; associated parameters (smoking, Hb, Sodium excretion in 24-hr Urine); ${ }^{\text {III }}$ Model III: adjustment for other factors (age, gender, eGFR); iv Model IV: full model (combination of model II and III). 
Ebrahimi et al. ${ }^{(11)}$ using Matlab (MathWorks Inc, Natick, Massachusetts, USA). The histogram of the $\mathrm{R}_{2}{ }^{*}$ map was calculated and fitted to a Gaussian function (cortex) and a gamma function (medulla) (Figure 1). To objectively differentiate medulla, collecting system and vessels, a semi-automatic filter was applied to the $\mathrm{R}_{2}{ }^{*}$ maps. High intensity voxels, representing collecting system, and low intensity voxels, representing blood vessels, were excluded, as well as the four adjacent voxels (Figure 1). To obtain a measure for hypoxia, the $\mathrm{R}_{2}{ }^{*}$ of the $10 \%$ most hypoxic voxels $\left(90^{\text {th }}\right.$ percentile of the histogram: P90) was also take into account (Figure 1).

\section{Data Analysis}

BOLD MRI data were analyzed by two investigators (EV and AB), blinded to patient information and other results from the standardized screening protocol. Data from ten patients were randomly selected to determine intra-reader reproducibility ( 5 datasets from each field strength). Inter-reader reproducibility was determined in 16 patients (8 per field strength).

About $8 \%$ of the total kidney volume was included in the coronal as well as in the transverse slices. It was chosen to use a weighted average $\mathrm{R}_{2}{ }^{*}$-value of the coronal and transverse slices. To combine data from the 1.5T and the 3.0 T scanner, z-scores for both field strengths were calculated and combined. A z-score is calculated for each individual as the obtained individual measurement minus the mean value of the group divided by the standard deviation around the group average ([Z score = (participant value $-\mu) / \sigma]$ ).

Estimated glomerular filtration rate (eGFR) was calculated on the basis of the CKD-epi formula. ${ }^{(12)}$ The systolic blood pressure (SBP) after administration of captopril was calculated as average of the measurements taken at 60 and 90 min after intake. Albuminuria was categorized as normal albumin excretion ( $<30 \mathrm{mg} / 24-$ hr), micro-albuminuria ( $\geq 30$ to $<300 \mathrm{mg} / 24$-hr) and macro-albuminuria ( $\geq 300 \mathrm{mg}$ / 24-hr). Percentage dipping of SBP during night-time was calculated as [(mean daytime SBP -mean night-time SBP)/mean daytime SBP] * 100\%. ${ }^{(13)}$ Subjects were subdivided into 2 groups: dippers (percentage < 10\%), non-dippers $(>10 \%) .{ }^{(14)}$

\section{Statistical Analysis}

All variables were reported as mean $\pm \mathrm{SD}$, median (range), or as a proportion when appropriate. The Intra-class correlation coefficient (ICC; two-way mixed model, absolute agreement) and the Bland Altman method (defining bias and variation by calculating the mean difference between the observers, the standard deviations of the differences and the $95 \%$ limits of agreement) were used to determine inter- and intrareader reproducibility.

Either the unpaired Students t-test or the chi-square test was used, when applicable, to compare baseline data between patients scanned on either the 1.5 -T or 
the 3.0-T MR.

The aim of the current study was to investigate the relation between kidney function, albumuniuria as well as direct and indirect variables of the RAAS (CCT, PAC, PRA) and SNS (non-dipping pattern of BP, catecholamines in urine) and renal $\mathrm{R}_{2}{ }^{*}$-values. To analyze the association of each independent variable of interest with $\mathrm{R}_{2}{ }^{*}$-values of the cortex, medulla and P9o (dependent variables), linear regression was used. In total 4 models were applied per independent variable of interest. Model I, a univariable model, model II adjusting for variables theoretically associated with renal oxygenation ( $\mathrm{Hb}, 24-\mathrm{hr}$ sodium excretion and smoking), model III adjusting for age, gender and eGFR when considered appropriate and model IV, a full model (combination of model II and III). All models are shown in Appendix 3. In case of a non-normal distribution of the residuals of the model, appropriate logarithmic transformations were applied (PAC, PRA and change in SBP after captopril [log (SBP before captopril/SBP after captopril)]).

A two sided $P$ value of <0.05 was considered to be statistically significant. All analyses were performed with the SPSS statistical package version 20 (IBM SPSS Data Collection, Chicago, IL).

\section{Results}

Seventy-five patients were included in the present analysis. Baseline characteristics are listed in Table 1 . The laboratory variables are shown in Table 2. Fifty-five patients were imaged using the 1.5 -T system, and 20 patients were imaged on the 3 -T system. There were no significant differences in characteristics between patients imaged at 1.5-T compared to 3.0-T.Inter- and intra-reader reproducibility of $\mathrm{R}_{2}{ }^{*}$-values of the cortex, medulla and P90, were considered good based on the ICC (range: 0.9570.999) as well as the Bland Altman method (see section 5.7.2).

From five patients (7\%) both coronal as well as transverse images were excluded from analysis. In five patients (7\%) only the coronal or transverse scan was excluded. Six datasets were excluded because of motion artifacts, one because of multiple cysts, two because of erroneous shimming and one because of multiple eddy current artifacts visible in the $\mathrm{R}_{2}^{*}$-map.

Table 3 lists mean $\mathrm{R}_{2}{ }^{*}$-values of the cortex, medulla and P9o for both field strengths. As expected, $\mathrm{R}_{2}{ }^{*}$-values were a factor 1.4 higher on the 3.0-T MR.

\section{Relations Between Kidney Function, Albuminuria and $\boldsymbol{R}_{2}{ }^{*}$}

In a multivariable model adjusting for variables theoretically associated with renal oxygenation, medullary, but not cortical $\mathrm{R}_{2}{ }^{*}$ was inversely related to kidney function (Table 4: $\beta$ :-0.02, 95\%CI:-0.03--0.003, p: 0.02). Neither cortical nor medullary $\mathrm{R}_{2}^{*}$ as associated with the presence of micro- or macro-albuminuria in a multivariable 
model adjusting for BOLD-related variables, age and gender.

\section{Relation Between Direct and Indirect Parameters of The SNS and Ren- al Oxygenation}

In a full multivariable model, medullary $\mathrm{R}_{2}{ }^{*}$ as well as P9o positively related to the presence of a non-dipping profile (Table 4: medulla: $\beta$ : 0.57, 95\%CI: 0.11-1.04, p: 0.02; P9o: $\beta$ : 0.0.60, 95\%CI: 0.11-1.10, p: 0.02). Cortical $\mathrm{R}_{2}{ }^{*}$ tended to positively relate to noradrenaline excretion in $24-\mathrm{hr}$ urine in a multivariable model adjusting for BOLD-associated factors (Table 4: $\beta$ : 0.002, 95\%CI: -0.0004-0.004, p: 0.11). However, after adjustment for age and gender, this relation was no longer present ( $\beta$ : 0.001, 95\%CI: -0.002-0.003, p: 0.50). This might be explained by the positive relation between age and noradrenaline excretion $(\beta: 3.78,95 \% \mathrm{CI}: 1.01-6.54, \mathrm{p}$ : 0.008).

\section{Relation Between Direct and Indirect Parameters of the RAAS and Renal Oxygenation}

In a full multivariable model, medullary $\mathrm{R}_{2}{ }^{*}$ tended to inversely relate to PAC determined in supine position (Table 4: $\beta$ : $-0.66,95 \% \mathrm{CI}:-1.41-0.10, \mathrm{p}: 0.09$ ). Moreover, in a full multivariable model, cortical $\mathrm{R}_{2}^{*}$ also tended to relate inversely to PRA levels determined in supine position (Table $4: \beta$ :-0.54, 95\%CI: -1.16-0.08, p: 0.09).

In a full multivariable model, cortical and medullary $\mathrm{R}_{2}^{*}$ as well as P9o, were positively related to the BP-lowering effect of captopril (Table 4 and Figure 2; cortex: $\beta$ : -8.40, 95\%CI: -15.35- -1.44, p:0.02; medulla: $\beta$ : -9.69 , 95\%CI: $-16.80-$ -2.58, p:0.008; P9o: $\beta$ : -9.18, 95\%CI: -16.85- -1.51, p:0.02).
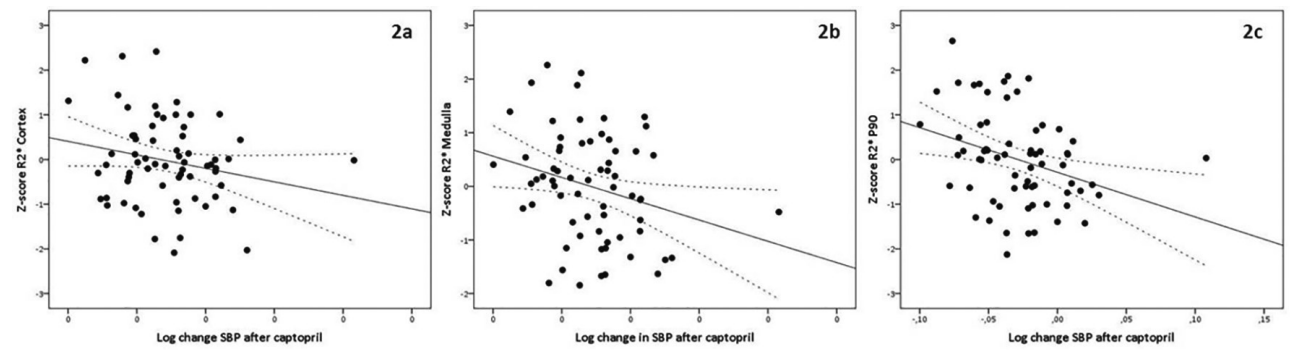

Figure 2. Relation between the BP-lowering effect of captopril and renal oxygenation; a change in SBP (mmHg) after captopril vs. cortical $\mathrm{R}_{2}{ }^{*}(\mathrm{z}$-score); $\mathbf{b}$ change in SBP (mmHg) after captopril vs. medullary $\mathrm{R}_{2}{ }^{*}(\mathrm{z}$-score); c change in SBP (mmHg) after captopril vs. P90 (z-score). Log change in SBP: $\log$ (SBP before captopril/SBP after captopril). 


\section{Discussion}

The present study shows in patients with hypertension that eGFR inversely relates to renal $\mathrm{R}_{2}{ }^{*}$. This can be interpreted as a positive relation between kidney function and renal oxygenation. The fact that we temporarily stopped antihypertensive medication, gave us the unique opportunity to perform an additional set of measurements. To the best of our knowledge, we are the first to report on the relation between direct and indirect variables of the RAAS and SNS and BOLD signal. We found that direct and indirect variables of the RAAS and SNS are associated with renal $\mathrm{R}_{2}{ }^{*}$. Some but not all of these relations support the idea that the presence of an activated RAAS and SNS is especially seen in patients with low oxygenation, quantified by BOLD MRI.

The relation between eGFR and BOLD variables is addressed before in a number of studies, but have produced mixed results. Almost all studies were done while medication was continued. This could be a limitation because BOLD signal and eGFR can be affected by the use of different antihypertensive drugs. Indeed, we recently showed that a RAAS inhibitor changed the BOLD signal in CKD patients. ${ }^{(15)}$ A specific strength of the present study is the wash-out of medication. Only one study investigating the relation between kidney function and renal oxygenation, quantified by the BOLD technique, excluded patients using medication. In that study, young hypertensive men ( $\mathrm{n}=8$, mean BP 142 $\pm 7 / 92 \pm 5 \mathrm{mmHg}$, GFR 106.9 $\pm 15.6 \mathrm{~mL} /$ $\left.\min / 1.73 \mathrm{~m}^{2}\right)$ and normotensive men $(\mathrm{n}=10$, mean BP $125 \pm 8 / 67 \pm 7 \mathrm{mmHg}$, normal eGFR) were compared. ${ }^{(16)}$ It was concluded that at any sodium intake, medullary oxygenation was slightly higher in hypertensive men compared with controls. The study population very much differs from our study; BP was considerably higher and eGFR lower in our study.

Many of the other studies investigating the relation between renal oxygenation using BOLD MRI and kidney function were done in diabetics. ${ }^{(17-20)}$ Our study is in line with the studies of Inoue et al. and Manotham et al. ${ }^{(20,21)}$ cInoue found a positive relation between eGFR and renal oxygenation in CKD patients without diabetes ( $\mathrm{n}=76$, mix of primary kidney disease, mean eGFR $46 \pm 36 \mathrm{~mL} / \mathrm{min} / 1.73 \mathrm{~m}^{2}$ ), but not in patients with diabetic nephropathy $\left(\mathrm{n}=43\right.$, mean eGFR $\left.44 \pm 28 \mathrm{~mL} / \mathrm{min} / 1.73 \mathrm{~m}^{2}\right){ }^{(20)}$ Manotham et al. found a lower medullary oxygenation in patients with CKD compared to healthy controls. ${ }^{(21)}$ The largest study available investigating the relation between renal BOLD and kidney function, is by Michaely et al. ${ }^{(22)}$ In total 400 patients undergoing abdominal MRI for various reasons were included in this study. From 280 of these patients, information on kidney function (KDOQI stage 1-5) was available. No relation was found between eGFR or stages of kidney function and renal oxygenation. Limitations of the study include the lack of information on medication use and on other clinical data.

The second interesting finding of our study is the association between renal 
BOLD MRI and indirect and direct parameters of the SNS. Medullary $\mathrm{R}_{2}{ }^{*}$ as well as P9o, a measure for the degree of hypoxia in the kidney, positively related to the presence of a non-dipping blood pressure profile. This can be interpreted as an inverse relation between medullary oxygenation and a non-dipping profile. A non-dipping profile was associated with increased activity of the SNS: the day-night BP difference was inversely related to sympathetic activity, assessed as muscle sympathetic nerve activity. ${ }^{(14)}$ Moreover, cortical $\mathrm{R}_{2}{ }^{*}$ tended to positively relate to the urinary excretion of noradrenaline. These results are in line with animal studies showing that renal ischemia triggers activation of the SNS. Even a small lesion by intrarenal injection of phenol chronically increases central sympathetic activity resulting in hypertension in rats, without affecting kidney function. ${ }^{(23)}$ The BP-elevation can be prevented by prior denervation of the phenol injected kidney ${ }^{(23)}$, which demonstrates a crucial role of intact renal innervation for afferent signaling from the kidney to the vasomotor center. Moreover, we have recently documented in this model that unilateral intrarenal phenol injection causes a profound vasoconstriction in the contralateral kidney that is prevented by denervation of the contralateral kidney. ${ }^{(24)}$ This finding supports a role for efferent signalling from vasomotor centre to kidney. Thus, the studies support the notion that both afferent and efferent signalling play a key role in this model of hypertension. ${ }^{(23,24)}$ Whether intrarenal phenol injection induces local renal hypoxia remains to be shown. It is hypothesized that renal hypoxia triggers sympathetic excitation by the release of adenosine. ${ }^{(25)}$ In dogs, infusion of adenosine into the renal artery causes an increase in sympathetic activity resulting in hypertension. ${ }^{(25)}$ Renal denervation though, prevents hypertension during infusion of adenosine. ${ }^{(25)}$

The third set of observations of our study, the relation between renal BOLD MRI and direct and indirect variables of the RAAS, produced contradictory results. Medullary $\mathrm{R}_{2}{ }^{*}$ tended to inversely relate to PAC and PRA levels determined in supine position, which can be interpreted as a positive relation between PAC and PRA with renal oxygenation. This seems contradictory to the hypothesis that the RAAS is one of the systems activated by the presence of renal hypoxia. ${ }^{(5)}$ However, in contrast to these findings, we also found that cortical and medullary $\mathrm{R}_{2}{ }^{*}$ as well as P9o were positively related to the BP-lowering effect of captopril, and in the full multivariable regression model these relations were still significant for the renal medulla. So, a greater BP-lowering effect of captopril is associated with lower renal oxygenation. ACE inhibitors work best in the presence of an activated RAAS. One of the effects of hypoxia is the activation of the RAAS. Both AngII as well as phenylephrine are known to reduce renal blood flow by increasing afferent and efferent arteriole resistance. (26,27) An important study supporting the effect of the RAAS on renal oxygenation is the study by Schachinger et al. This study in 6 healthy volunteers showed that intravenously administered AngII reduces renal oxygenation as determined with 
BOLD, while noradrenaline and nitroprusside, with comparable changes in BP, did not alter renal BOLD signal. ${ }^{(28)}$ A non-hemodynamic effect of AngII is therefore thought to be involved in this reduction in renal oxygenation by AngII. Also by inhibiting the RAAS, directly measured renal oxygen levels in spontaneously hypertensive rats improved by candesartan and to a much lesser extent by non-RAAS inhibiting drugs with a comparable BP-lowering effect, which suggests a hemodynamic independent effect of candesartan. ${ }^{(29)}$ We reported earlier that a RAAS inhibitor changed BOLD signal in CKD patients, whereas it had no effect in healthy controls. ${ }^{(15)}$

Our findings very well fit in the 'chronic hypoxia hypothesis' as proposed by Fine et al. ${ }^{\left({ }^{\circ}\right)}$ Several animal studies have shown a relation between renal hypoxia and CKD. ${ }^{(31)}$ Renal ischemia is proposed to lead to a vicious circle of tissue fibrosis, pursuant obliteration of the renal microvasculature and continued damage. ${ }^{\left({ }^{2}\right)}$ Also in several animal models of hypertension, cortical as well as medullary oxygenation is lower in hypertensive animals as compared to controls. ${ }^{\left({ }^{3}\right)}$ Renal hypoxia is thought to both be a result as well as a cause of hypertension, potentially inducing a vicious cycle.

Most of the relations were found with medullary oxygenation. This can be due to the fact that there are indeed only such relations with the poorly oxygenated medulla. However, it should be noted that redox balance in the renal medulla is crucial for determining BP. ${ }^{(34)}$ Thus, theoretically, changes in $\mathrm{pO}_{2}$ that are restricted to the medulla may be crucial in the pathogenesis of both hypertension and, ultimately, CKD. Another explanation can be that BOLD MRI is less sensitive to changes in cortical oxygenation, because cortical blood $\mathrm{pO}_{2}$ lies on the shoulder of the hemoglobin oxygenation curve. Medullary oxygen level however lies on the linear part of the curve, and is therefore more sensitive to small changes in oxygen tension. So, larger differences in oxygenation are necessary to observe similar changes in the $\mathrm{R}_{2}{ }^{*}$ for the cortex compared with the medulla. ${ }^{\left({ }^{3}\right)}$ In case of renal injury, hyperfiltration and hypertrophy of uninjured nephrons can take place. These nephrons have a high metabolic demand, in contrast with injured nephrons. As a consequence there can be a heterogeneous distribution of hypoxia in the kidney, which cannot be detected using average $\mathrm{R}_{2}^{*}$. P90 gives information on the amount of hypoxia ( $9 \mathrm{O}^{\text {th }}$ percentile of the histogram). Moreover, it has recently been shown that renal hypoxia, attributable to increased oxygen consumption, can induce nephropathy independently of hyperglycemia and oxidative stress. ${ }^{\left({ }^{36)}\right)}$ Thus, when renal injury is present, compensatory hypertrophy of remaining nephrons can lead to a vicious cycle of hypoxia, and further loss of viable nephrons.

Several limitations need to be mentioned. Assessment of oxygenation of the kidneys, either by direct measurement or indirectly by BOLD MRI, does not distinguish between (changes in) oxygen supply and consumption. It is therefore not possible to investigate the association between renal blood flow or oxygen 
consumption (mostly influenced by tubular transport) and the variables investigated in the present study. Further, a specific limitation of our study can be the use of two different field strengths. To combine the data, we used z-scores. Finally, this is a cross-sectional study not allowing us to draw conclusions on cause-effect relations. Longitudinal research on the relation between eGFR and BOLD is crucial.

In conclusion, our study shows that renal $\mathrm{R}_{2}{ }^{*}$ inversely related to kidney function in patients with hypertension, giving support to the idea that CKD is associated with the presence of renal ischemia. Medullary $\mathrm{R}_{2}{ }^{*}$ positively related to the BP-lowering effect of captopril, the presence of a non-dipping profile, an indirect measure of sympathetic activity, and to the urinary excretion of noradrenaline. Some but not all data support the idea that the presence of an activated RAAS and SNS is especially seen in patients with low oxygenation, quantified by the BOLD technique. 


\section{References}

1. Siddiqi L, Joles JA, Grassi G, Blankestijn PJ. Is kidney ischemia the central mechanism in parallel activation of the renin and sympathetic system? J Hypertens 2009;27:1341-1349.

2. Reid IA. Interactions between ANG II, sympathetic nervous system, and baroreceptor reflexes in regulation of blood pressure. Am J Physiol 1992;262:E763-778.

3. O'Connor PM, Kett MM, Anderson WP, Evans RG. Renal medullary tissue oxygenation is dependent on both cortical and medullary blood flow. Am J Physiol Renal Physiol 2006;290:F688-694.

4. Li LP, Halter S, Prasad PV. Blood oxygen level-dependent MR imaging of the kidneys. Magn Reson Imaging Clin N Am 2008;16:613-625, viii.

5. Heyman SN, Khamaisi M, Rosen S, Rosenberger C. Renal parenchymal hypoxia, hypoxia response and the progression of chronic kidney disease. Am J Nephrol 2008;28:9981006.

6. Verloop WL, Vink EE, Voskuil M, et al. Eligibility for percutaneous renal denervation: the importance of a systematic screening. J Hypertens 2013;31:1662-1668.

7. Funder JW, Carey RM, Fardella C, et al. Case detection, diagnosis, and treatment of patients with primary aldosteronism: an endocrine society clinical practice guideline. $\mathrm{J}$ Clin Endocrinol Metab 2008;93:3266-3281.

8. Shionoiri H, Yasuda G, Yoshimura H, et al. Antihypertensive effects and pharmacokinetics of single and consecutive administration of doxazosin in patients with mild to moderate essential hypertension. J Cardiovasc Pharmacol 1987;10:90-95.

9. Mancia G, Fagard R, Narkiewicz K, et al. 2013 ESH/ESC Guidelines for the management of arterial hypertension: the Task Force for the management of arterial hypertension of the European Society of Hypertension (ESH) and of the European Society of Cardiology (ESC). J Hypertens 2013;31:1281-1357.

10. Lyons DF, Kem DC, Brown RD, Hanson CS, Carollo ML. Single dose captopril as a diagnostic test for primary aldosteronism. J Clin Endocrinol Metab 1983;57:892-896.

11. Ebrahimi B, Gloviczki M, Woollard JR, Crane JA, Textor SC, Lerman LO. Compartmental analysis of renal BOLD MRI data: introduction and validation. Invest Radiol 2012;47:175182.

12. Levey AS, Stevens LA, Schmid CH, et al. A new equation to estimate glomerular filtration rate. Ann Intern Med 2009;150:604-612.

13. Urbina E, Alpert B, Flynn J, et al. Ambulatory blood pressure monitoring in children and adolescents: recommendations for standard assessment: a scientific statement from the American Heart Association Atherosclerosis, Hypertension, and Obesity in Youth Committee of the council on cardiovascular disease in the young and the council for high blood pressure research. Hypertension 2008;52:433-451. 
14. Grassi G, Seravalle G, Quarti-Trevano F, et al. Adrenergic, metabolic, and reflex abnormalities in reverse and extreme dipper hypertensives. Hypertension 2008;52:925931.

15. Siddiqi L, Hoogduin H, Visser F, Leiner T, Mali WP, Blankestijn PJ. Inhibition of the renin-angiotensin system affects kidney tissue oxygenation evaluated by magnetic resonance imaging in patients with chronic kidney disease. J Clin Hypertens (Greenwich) 2014;16:214-218.

16. Pruijm M, Hofmann L, Maillard M, et al. Effect of sodium loading/depletion on renal oxygenation in young normotensive and hypertensive men. Hypertension 2010;55:11161122.

17. Epstein FH, Veves A, Prasad PV. Effect of diabetes on renal medullary oxygenation during water diuresis. Diabetes Care 2002;25:575-578.

18. Yin WJ, Liu F, Li XM, et al. Noninvasive evaluation of renal oxygenation in diabetic nephropathy by BOLD-MRI. Eur J Radiol 2012;81:1426-1431.

19. Wang ZJ, Kumar R, Banerjee S, Hsu CY. Blood oxygen level-dependent (BOLD) MRI of diabetic nephropathy: preliminary experience. J Magn Reson Imaging 2011;33:655-660.

20. Inoue $\mathrm{T}$, Kozawa $\mathrm{E}$, Okada $\mathrm{H}$, et al. Noninvasive evaluation of kidney hypoxia and fibrosis using magnetic resonance imaging. J Am Soc Nephrol 2011;22:1429-1434.

21. Manotham K, Ongvilawan B, Urusopone P, et al. Angiotensin II receptor blocker partially ameliorated intrarenal hypoxia in chronic kidney disease patients: a pre-/post-study. Intern Med J 2012;42:e33-37.

22. Michaely HJ, Metzger L, Haneder S, Hansmann J, Schoenberg SO, Attenberger UI. Renal BOLD-MRI does not reflect renal function in chronic kidney disease. Kidney Int 2012;81:684-689.

23. Ye S, Gamburd M, Mozayeni P, Koss M, Campese VM. A limited renal injury may cause a permanent form of neurogenic hypertension. Am J Hypertens 1998;11:723-728.

24. Koeners MP, Vink EE, Kuijper A, et al. Stabilization of hypoxia inducible factor-1alpha ameliorates acute renal neurogenic hypertension. J Hypertens 2014;32:587-597.

25. Katholi RE, Whitlow PL, Hageman GR, Woods WT. Intrarenal adenosine produces hypertension by activating the sympathetic nervous system via the renal nerves in the dog. J Hypertens 1984;2:349-359.

26. Blantz RC, Konnen KS, Tucker BJ. Angiotensin II effects upon the glomerular microcirculation and ultrafiltration coefficient of the rat. J Clin Invest 1976;57:419-434.

27. Myers BD, Deen WM, Brenner BM. Effects of norepinephrine and angiotensin II on the determinants of glomerular ultrafiltration and proximal tubule fluid reabsorption in the rat. Circ Res 1975;37:101-110.

28. Schachinger H, Klarhofer M, Linder L, Drewe J, Scheffler K. Angiotensin II decreases the renal MRI blood oxygenation level-dependent signal. Hypertension 2006;47:1062-1066.

29. Welch WJ, Baumgartl H, Lubbers D, Wilcox CS. Renal oxygenation defects in the spontaneously hypertensive rat: role of AT1 receptors. Kidney Int 2003;63:202-208. 
30. 31. Fine LG, Orphanides C, Norman JT. Progressive renal disease: the chronic hypoxia hypothesis. Kidney Int Suppl 1998;65:S74-78.

31. Fine LG, Norman JT. Chronic hypoxia as a mechanism of progression of chronic kidney diseases: from hypothesis to novel therapeutics. Kidney Int 2008;74:867-872.

32. Fine LG, Bandyopadhay D, Norman JT. Is there a common mechanism for the progression of different types of renal diseases other than proteinuria? Towards the unifying theme of chronic hypoxia. Kidney Int Suppl 2000;75:S22-26.

33. Welch WJ. Intrarenal oxygen and hypertension. Clin Exp Pharmacol Physiol 2006;33:1002-1005.

34. Cowley AW, Jr. Renal medullary oxidative stress, pressure-natriuresis, and hypertension. Hypertension 2008;52:777-786.

35. Prasad PV. Evaluation of intra-renal oxygenation by BOLD MRI. Nephron Clin Pract 2006;103:c58-65.

36. Friederich-Persson M, Thorn E, Hansell P, Nangaku M, Levin M, Palm F. Kidney hypoxia, attributable to increased oxygen consumption, induces nephropathy independently of hyperglycemia and oxidative stress. Hypertension 2013;62:914-919. 



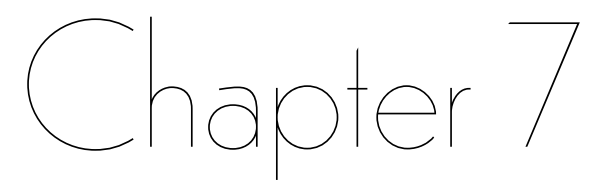

\section{Validation of Multiparametric MRI by Histopathology atter Nephrectomy: a Case Study}

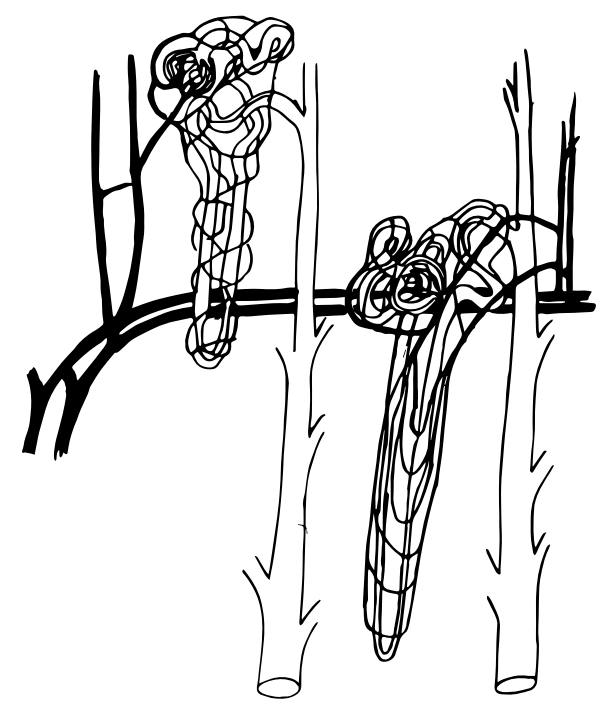

Author contributions

de Boer A*, Pieters TT*, Harteveld AA, Blankestijn PJ, Bos C, Froeling M, Goldschmeding R, Hoogduin JM, Joles JA, Petri BJ ,Verhaar MC, Leiner T, Nguyen TQ, van Zuilen AD ( ${ }^{*}$ authors contributed equally)

Published in

Magn Reson Mater Phy 2020; doi: 10.1007/s10334-020-00887-9. 


\section{Abstract}

Object Renal multiparametric MRI (mpMRI) is a promising tool to monitor renal allograft health to enable timely treatment of chronic allograft nephropathy. This study aims to validate mpMRI by whole-kidney histology following transplantectomy.

Materials and methods A patient with kidney transplant failure underwent mpMRI prior to transplantectomy. The mpMRI included blood oxygenation leveldependent (BOLD) MRI, T1 and T2 mapping, diffusion weighted imaging (DWI), 2D phase contrast (2DPC) and arterial spin labeling (ASL). Parenchymal mpMRI measures were compared to normative values obtained in 19 healthy controls. Differences were expressed in standard deviations (SD) of normative values. The mpMRI measures were compared qualitatively to histology.

Results The mpMRI showed a heterogeneous parenchyma consistent with extensive interstitial hemorrhage on histology. A global increase in T1 (+3.0SD) and restricted diffusivity (-3.6SD) were consistent with inflammation and fibrosis. Decreased T2 (-1.8SD) indicated fibrosis or hemorrhage. ASL showed diminished cortical perfusion $(-2.9 \mathrm{SD})$ with patent proximal arteries. 2DPC revealed a $69 \%$ decrease in renal perfusion. Histological evaluation showed a dense inflammatory infiltrate and fibrotic changes, consistent with mpMRI results. Most interlobular arteries were obliterated while proximal arteries were patent, consistent with ASL findings.

Discussion mpMRI findings correlated well with histology both globally as well as locally. 


\section{Introduction}

Kidney transplantation is the treatment of choice for patients with end-stage kidney disease $^{(1)}$. Close monitoring of the graft is crucial for early detection of treatable conditions ${ }^{(2)}$. Traditional methods of graft monitoring like the measurement of plasma creatinine and proteinuria only deviate when the majority of nephrons are irreversibly lost. Some centers use protocol biopsies to detect subclinical disease. However, transplant biopsies are invasive, carry the risk of sampling errors and are associated with a small risk of graft $\operatorname{loss}^{(3)}$. Therefore, protocol biopsies are not standard of care in all centers and are not suitable for monitoring disease progression. Multiparametric kidney MRI may help overcome these problems. MRI is sensitive to various relevant functional and structural markers like perfusion, oxygenation and fibrosis ${ }^{(4)}$. It can map the kidneys as a whole and is non-invasive, making it a suitable candidate for monitoring of local and global disease progression. For example, $\mathrm{T}_{1}$ and the apparent diffusion coefficient (ADC) correlate with fibrosis on histology and could be used to predict current graft dysfunction and future eGFR decline ${ }^{(5-7)}$. Perfusion measurements can be performed without contrast agent by using arterial spin labeling (ASL) and have been shown to correlate with reduced capillary density ${ }^{(8,7,9)}$.

Several histological findings in protocol biopsies, like interstitial fibrosis and tubular atrophy (IF/TA) and capillary rarefaction, strongly correlate to graft survival $[10,11]$. Both capillary rarefaction and IF/TA may be caused by a treatable condition, such as subclinical rejection or return of the original disease.

In our center, a multiparametric renal MRI protocol has recently been developed and evaluated ${ }^{(9)}$. The protocol consists of relaxometry ( $\mathrm{T}_{1}$ and $\mathrm{T}_{2}$ mapping), BOLD MRI, DWI, ASL and 2D phase contrast (2DPC) to measure renal blood flow (RBF). Normative values are available in 19 healthy volunteers ${ }^{(9)}$.

Previous studies on correlation of MRI measures with histology used kidney biopsies for histological validation, but given the often heterogeneous distribution of damage, biopsies might not be representative for the entire kidney. We recently had the unique opportunity to perform multiparametric MRI in a patient scheduled for allograft explantation, which allowed for a comparison of MRI findings to whole-kidney histology and to evaluate MRI performance in identifying intraorgan heterogeneity . The objective of this study was to validate the comprehensive MRI protocol to whole-kidney histology using 1) a comparison of MRI findings to normative values in healthy controls and 2) a comparison between whole-kidney histology and local as well as global changes in MRI measures. 


\section{Case Description}

The patient was a 46-year old female with an extensive history of liver fibrosis and end stage kidney disease of unknown origin. Eleven years ago, she underwent a kidney transplantation with a kidney donated after circulatory death. Several creatinine rises in the following years were attributed to tacrolimus toxicity and acute rejection. Seven years post-transplantation, the patient started hemodialysis because of chronic rejection, likely caused by non-adherence to the immunosuppressive regimen. Eleven years post-transplantation, chronic hematuria and onset of new HLA-antibodies suggested ongoing rejection for which she was treated with prednisolone. An urologic analysis did not suggest other causes for hematuria. Because of persistent pain in the region of the transplant kidney and persistent hematuria, it was decided to explant the allograft. During surgery, the kidney had to be cut from the surrounding capsule which led to minor superficial lesions and hemorrhaging.

Prior to surgery, informed consent for inclusion in an ongoing study on multparametric MRI in transplant kidneys was obtained and the MRI was scheduled 10 days before transplantectomy.

\section{Materials and Methods}

\section{MRI acquisition and processing}

The MRI examination was performed on a 3 T MR system (Ingenia, Philips Healthcare, Eindhoven, the Netherlands; software release 5.3.1). The patient was asked to avoid salt- and protein rich meals and to drink $2 \mathrm{~L}$ per 24 hour of non-alcoholic liquids on the day of the scan to roughly standardize hydration and dietary conditions. The scan protocol, MR system and scan conditions were the same as described earlier ${ }^{(9)}$, therefore the baseline results of that study could be used as normative values.

The scan protocol is described in detail elsewhere ${ }^{(9)}$. In short, it consisted of localizer images followed by an anatomical $\mathrm{T}_{1}$ weighted Dixon. BOLD $/ \mathrm{R}_{2}{ }^{*}$ mapping was performed with a 15-echo gradient echo sequence. Diffusion weighted imaging was performed with a set of b-values and directions per b-value that allowed for both a DTI and an IVIM analysis. $T_{1}$ mapping was performed with a slice-cycled inversion recovery sequence and $T_{2}$ mapping was achieved using $T_{2}$ preparations. Single slice 2D phase contrast allowed for quantification of blood velocity in the (transplant) renal artery. ASL was performed using a flow-attenuated inversion recovery scheme with 4 different inversion times. Total scan time was approximately 1 hour. Scan parameters are presented in Table 1.

Processing involved post-hoc motion correction, fitting of the appropriate model and delineation of regions of interest (ROIs), avoiding areas affected by artefacts. Quality of all scans was assessed visually by an expert reader (AB, 5 years 


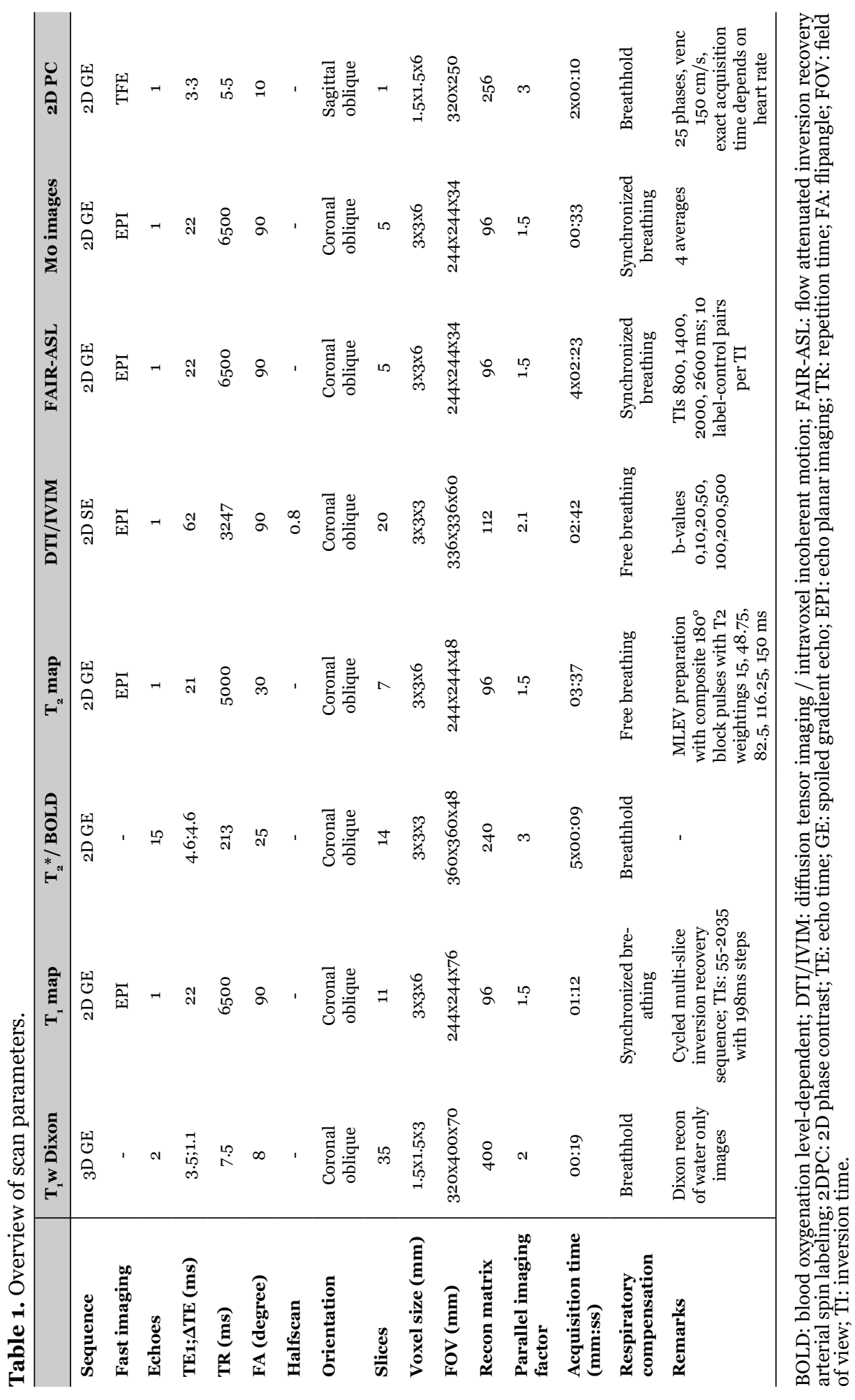


of experience in renal imaging) and scans of insufficient quality as assessed by visual inspection were excluded from analysis. In the patient, no discrimination between cortex and medulla was possible. Therefore, whole-parenchyma ROIs were used to allow for comparison between the patient and controls. ROI generation was semiautomated, using a combination of thresholding and k-means clustering. ${ }^{(9)}$ For ROI generation in the graft, manual intervention was required since the normal anatomy was virtually lost. The collecting system could be identified on the anatomical scans and was excluded. The remaining parenchyma was entirely included. For detailed information on post-processing, see ${ }^{(9)}$.

After processing, several parameter maps were obtained. For relaxometry, $\mathrm{T}_{1}$, $\mathrm{T}_{2}$ and $\mathrm{R}_{2}^{*}$ maps were generated. For the DWI data, both a diffusion tensor imaging (DTI) and intravoxel incoherent motion (IVIM) analysis was performed. DTI analysis yielded the fractional anisotropy (FA) and the mean diffusivity (MD). IVIM enabled measurement of the contribution of microvascular perfusion (perfusion fraction, $\mathrm{PF}$ ) to the diffusion coefficient (D). Local perfusion was measured with ASL MRI using four different inversion times, allowing both quantification of perfusion and determination of arterial transit times (ATT). 2D phase contrast (2DPC) MRI was used to measure total blood flow through the renal artery.

From the $\mathrm{R}_{2}{ }^{*}$ map (BOLD) and the $\mathrm{T}_{2}$ map, $\mathrm{R}_{2}$ ' could be calculated:

$$
R_{2}^{\prime}=R_{2}^{*}-\frac{1}{T_{2}}
$$

Here, $\mathrm{R}_{2}{ }^{\prime}, \mathrm{R}_{2}{ }^{*}$ and $\mathrm{T}_{2}$ are the median values of the parenchymal ROIs. Compared to $\mathrm{R}_{2}^{*}, \mathrm{R}_{2}$ ' is considered to reflect oxygenation more directly since it is not influenced by changes in $\mathrm{T}_{2}$.

\section{Histology}

After fixation in formalin, coronal sections of the explanted kidney were obtained from the upper pole (ventral, dorsal and lateral side), lower pole (ventral, dorsal and lateral side) and hilar region (ventral and dorsal side). In addition, we added a nephrectomy sample from a tumor nephrectomy where we show the healthy tissue as comparison to the explanted graft. Sections were paraffin-embedded.Next, slides were cut at $3 \mu \mathrm{m}$ and stained with haematoxylin and eosin (H\&E), periodic acidSchiff (PAS) and methenamine silver (Jones' stain) using standard protocols. Finally, slides were analyzed by two experienced renal pathologists (RG, TQN).

\section{Statistical Analysis}

MRI measures of the entire parenchyma were not normally distributed due to substantial differences between cortex and medulla in healthy kidneys. Therefore, median values and interquartile ranges (IQR) were calculated for the entire 
parenchyma ROI analysis. To assess changes in texture, local standard deviation (SD) maps were calculated where each voxel contains the SD of the 3x3 surrounding voxels. The median values within the ROI on these local SD maps were reported as well. For healthy subjects, the group-wise mean and SD were reported for all measures. Differences between the patient's values and normative values were expressed as percentage differences with respect to the normative values and in SDs of the normative values. Since only one patient was analyzed, no comparative statistical tests were performed.

To compare the voxel distribution within the parameter maps between healthy and the diseased kidney, histograms were used. For each examination in each subject, a histogram was calculated. In the controls, the median histogram was calculated. Parameter variation within controls were demonstrated by the IQR, the 10-90 and 2.5-97.5 inter-percentile ranges and are shown as shaded areas on the histograms. Results of histology were described qualitatively. The comparison between histopathology and MRI findings was performed in a qualitative way by an experienced renal pathologist (TQ) and an author with 5 years of experience in renal $\operatorname{MRI}(\mathrm{AB})$.

\section{Ethics}

This study was performed according to the declaration of Helsinki and ethical guidelines of our institution. The clinical and research activities being reported are consistent with the Principles of the Declaration of Istanbul as outlined in the 'Declaration of Istanbul on Organ Trafficking and Transplant Tourism'. The histology shown in this study was collected as part of routine clinical care. The protocol used in this study was approved by the institutional review board of our center. The patient signed informed consent for the acquisition of the MRI and use of the data. Control data was acquired as part of a separate study, which was approved be the institutional review board. All control subjects signed informed consent.

\section{Results}

Data of nineteen healthy subjects with a median age of 49 (IQR 45-57) were used as normative values. The patient tolerated the MRI examination well and all data were of sufficient quality to be included in the current analysis.

\section{MRI - Comparison to Normative Values}

In Figures 1 and 2, the MRI parameter maps belonging to the patient are shown alongside corresponding maps native kidney maps of a single control. For almost all MR measurements, noticeable differences could be appreciated between the patient and the control. 

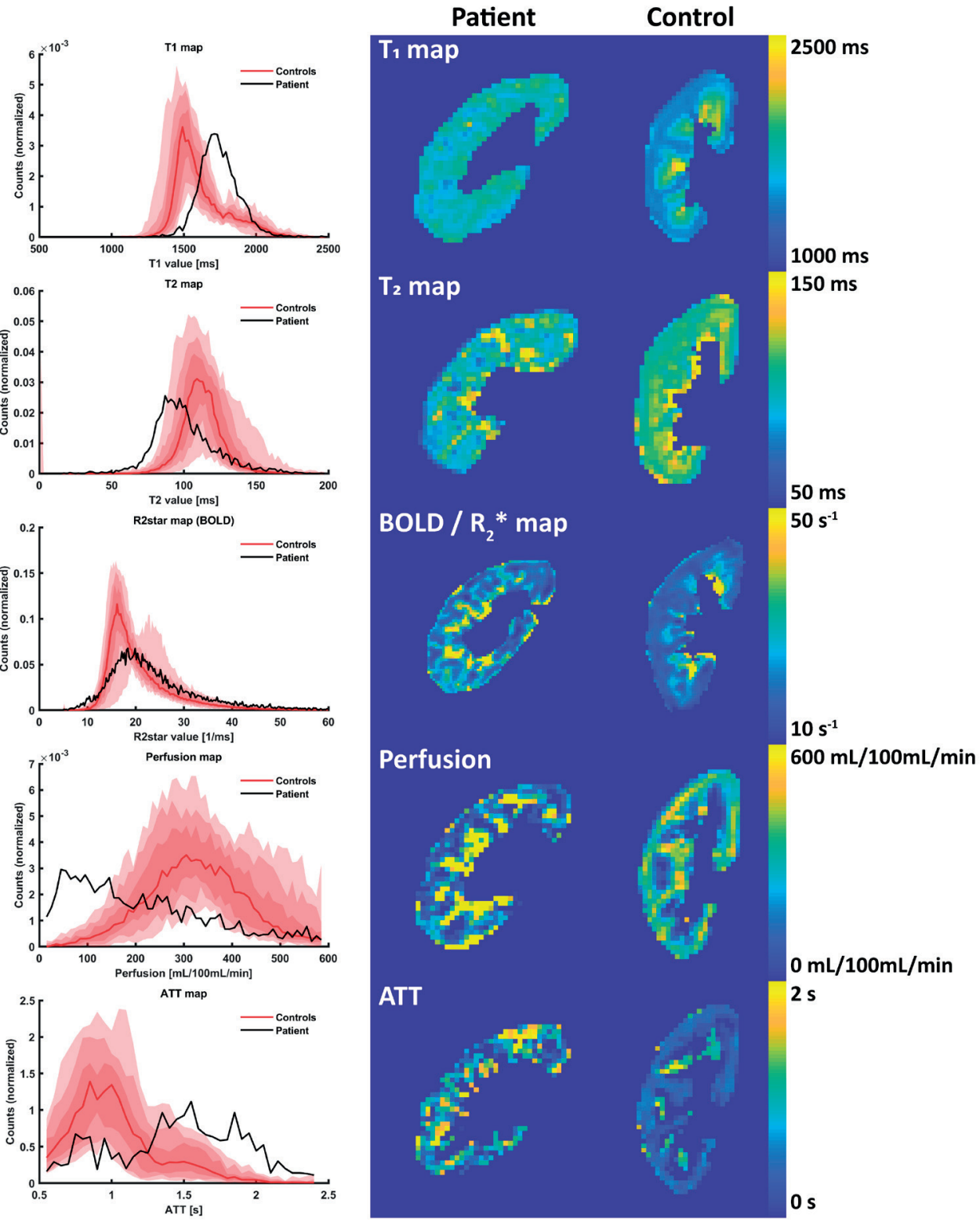

Figure 1. Histograms (left) and parameter maps for the patient (middle column) and a single control (right) for $\mathrm{T}_{1}$ and $\mathrm{T}_{2}$ mapping, BOLD and perfusion and ATT as assessed by ASL. The histograms show the voxel distribution in the patient and the median voxel distribution in controls, along with the spread between controls. The shaded areas denote the interquartile range, the 10-90 and the 2.5-97.5 interpercentile range (dark to lightly shaded). The histograms are normalized.

BOLD: blood oxygenation level-dependent MRI; ATT: arterial transit time; ASL: arterial spin labelling. 

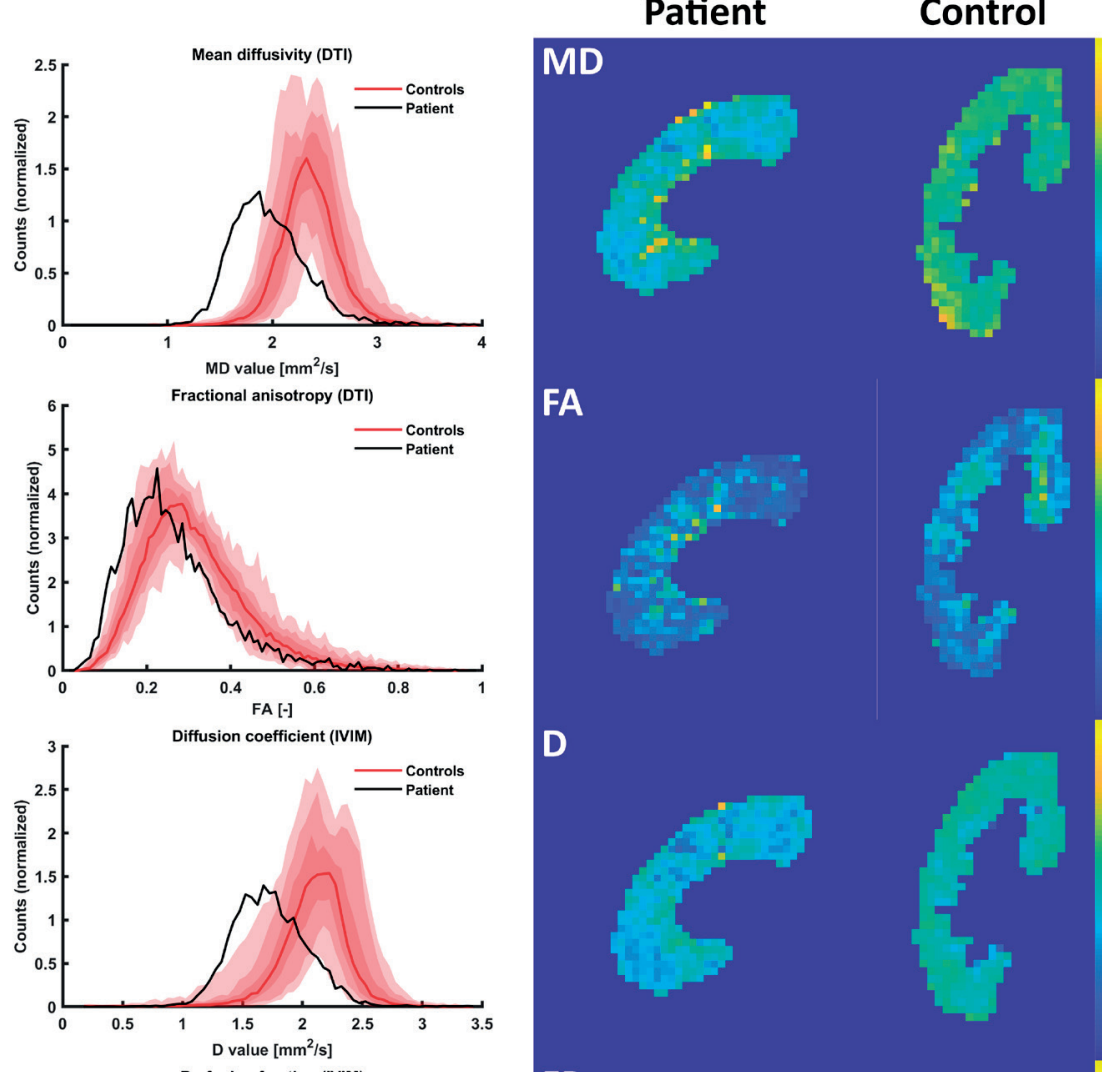

$2 \mathrm{~mm}^{2} / \mathrm{s}$
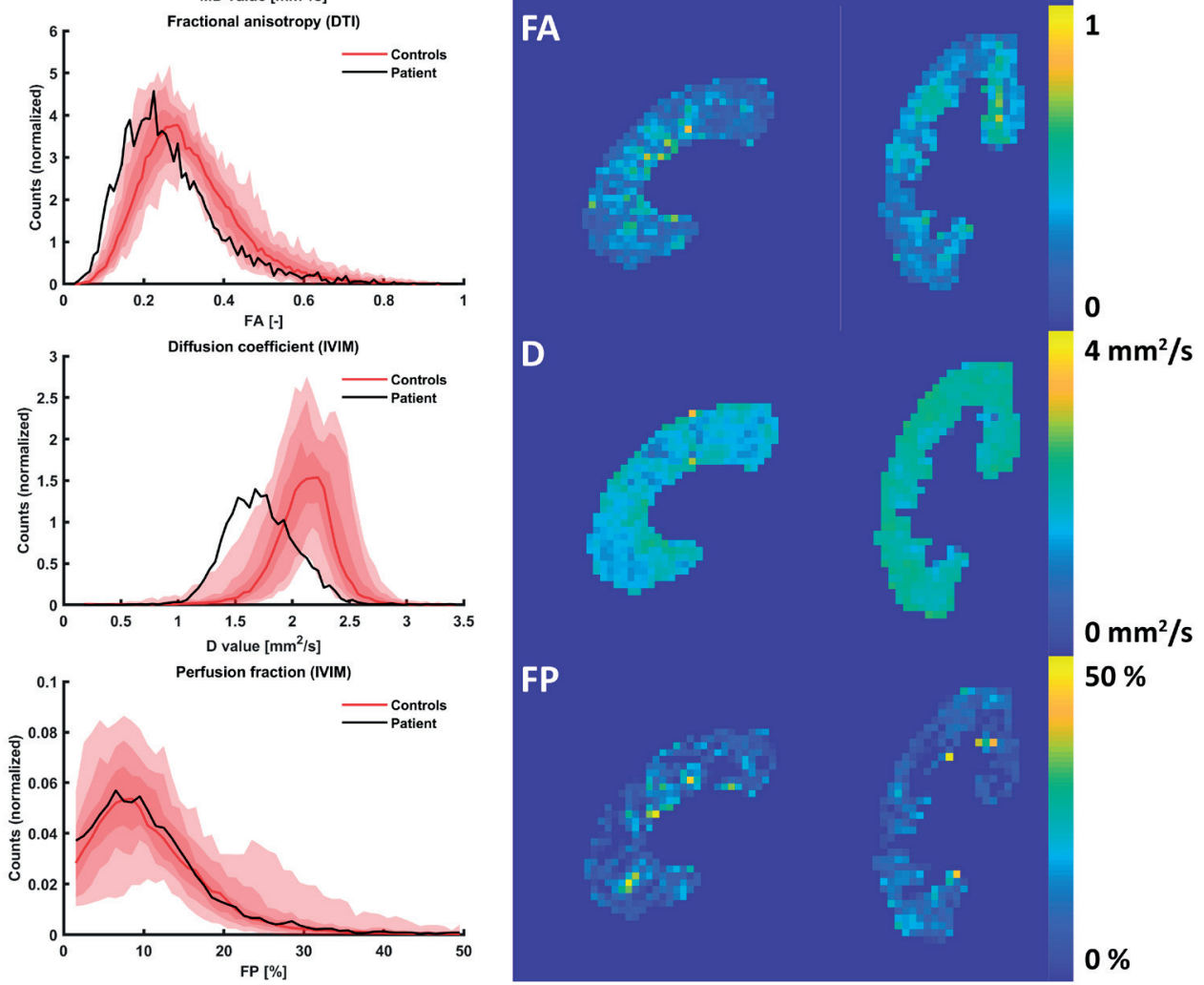

D

$4 \mathrm{~mm}^{2} / \mathrm{s}$
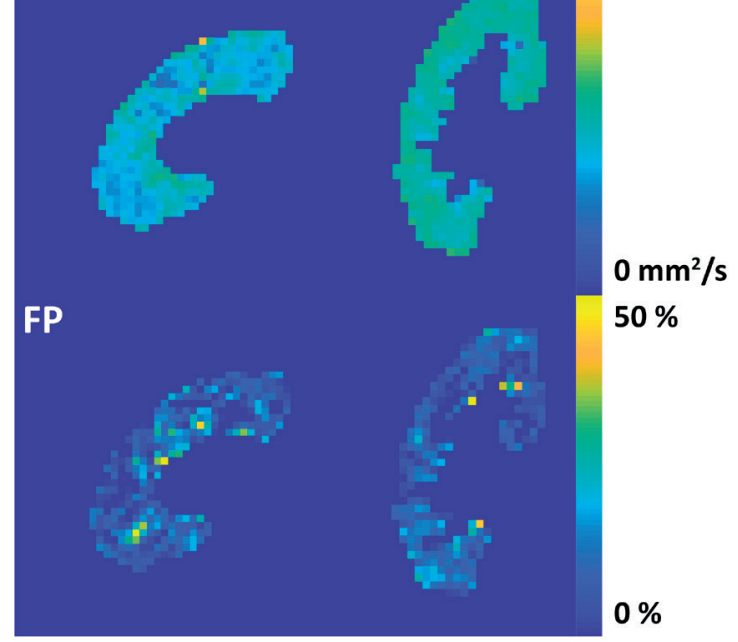

$50 \%$

$0 \%$

Figure 2. Histograms (left) and parameter maps for the patient (middle column) and a control (right) for all diffusion measures: MD and FA from the DTI analysis and D and PF from the IVIM analysis. The histograms show the voxel distribution in the patient and the median voxel distribution in controls, along with the spread between controls.

MD: mean diffusivity; FA: fractional anisotropy; D: diffusion coefficient; $P F$ : perfusion fraction. 
In healthy subjects, the cortex and medulla could be easily discriminated thanks to the higher $\mathrm{T}_{1}$ in the medulla. Within the cortex and medulla, the $\mathrm{T}_{1}$ was rather homogeneous. In the patient however, there was no difference between cortex and medulla and the $T_{1}$ values within the parenchyma were heterogeneous (Figure 1). However, due to the loss of corticomedullary differentiation, this was not reflected in the IQR (167 ms vs 213 (37) ms, Table 2) nor in the local SD (91 vs 96 (16) ms). Overall, $\mathrm{T}_{1}$ was increased in the diseased kidney due to microstructural changes in the tissue, most likely fibrosis and influx of inflammatory cells (Table 2).

The parenchymal $\mathrm{T}_{2}$ of the diseased kidney was decreased as compared to controls (Table 2). In Figure 1 marked heterogeneity was seen throughout the parenchyma reflected by large increase in both local SD (14.5 vs $7.8(1.3) \mathrm{ms})$ and IQR (25 vs 15 (3) ms) in the diseased kidney. Regionally, $\mathrm{T}_{2}$ approached $150 \mathrm{~ms}$, which is the upper cut-off value of the fit. $\mathrm{T}_{2}$ values in this range indicate high water content, for example edema or fluid collections. Due to fixation, this could not be confirmed histologically.

The BOLD or $\mathrm{R}_{2}{ }^{*}$ map confirms the finding of increased heterogeneity, which was also reflected in the broader histogram compared to the controls (Figure 1), the increased IQR (10.8 vs 7.1 (1.4) $\mathrm{s}^{-1}$ ) and the marked increase in local SD (3.9 vs 2.4 $\left.(0.4) \mathrm{s}^{-1}\right)$. No marked difference in global median $\mathrm{R}_{2}{ }^{*}$ value between the patient and healthy subjects was seen (Table 2).

Regarding perfusion, in healthy subjects areas with high perfusion were located in the cortex, but in the patient these areas were located more centrally, corresponding to the locations of large vessels (Figure 3). Around patent large vessels, perfusion was very high, while other areas seemed devoid of any blood supply. In healthy subjects, the latter was only seen in the inner medulla. The arterial transit times also showed a heterogeneous pattern in the diseased kidney (IQR 0.99 vs 0.43 (0.15)), compared to homogeneous and relatively short arrival times in the healthy cortex. Overall, 2DPC showed a marked decrease in blood flow through the renal artery in the diseased kidney (140 vs 422 (159) $\mathrm{mL} / \mathrm{min}$ ).

Results of the diffusion analysis are shown in Figure 2. Both MD and D showed higher restriction of diffusion compared to healthy subjects (Table 2), consistent with fibrosis and inflammation. A slight increase in heterogeneity was seen in the patient in all diffusion measures. FA was slightly decreased indicating less anisotropy, while no clear difference was seen between the patient and the controls for the PF. However, it should be noted that the quality of PF map was lower than the other parameter maps. 
Table 2. MRI measures for the patient compared to controls. Median values, interquartile ranges and the local SDs of whole parenchyma ROIs are reported. For the controls, the groupwise mean (standard deviation) of the ROI medians, IQRs and local SDs are calculated. Percentage difference between patient and controls with respect to the controls was calculated, as well as the absolute difference in units of the SD of the distribution in healthy volunteers.

\begin{tabular}{|c|c|c|c|c|c|}
\hline & & Patient & Controls & $\begin{array}{l}\text { Difference } \\
\text { (\%) }\end{array}$ & $\begin{array}{l}\text { Difference } \\
\text { (SD) }\end{array}$ \\
\hline \multirow[t]{3}{*}{$T_{1} \operatorname{map}(\mathrm{ms})$} & Median & 1732 & $1556(59)$ & +11 & +3.0 \\
\hline & IQR & 167 & $213(37)$ & -21 & -1.2 \\
\hline & Local SD & 91 & $96(16)$ & -5 & -0.3 \\
\hline \multirow[t]{3}{*}{$T_{2} \operatorname{map}(\mathrm{ms})$} & Median & 97 & $113(8)$ & -14 & -1.8 \\
\hline & IQR & 25 & $15(3)$ & +67 & +3.9 \\
\hline & Local SD & 14.5 & $7.8(1.3)$ & +87 & +5.1 \\
\hline \multirow[t]{3}{*}{$\mathbf{R}_{2}^{*} \operatorname{map} / \operatorname{BOLD}\left(\mathbf{s}^{-1}\right)$} & Median & 21.7 & $19.0(2.1)$ & +15 & +1.3 \\
\hline & IQR & 10.8 & $7.1(1.4)$ & +52 & +2.5 \\
\hline & Local SD & 3.9 & $2.4(0.4)$ & +61 & +4.0 \\
\hline R2' (BOLD and T2 map) $\left(\mathrm{s}^{-1}\right)$ & Median & 11.4 & $10.1(1.9)$ & +13 & +0.7 \\
\hline \multirow[t]{3}{*}{ ASL - perfusion $(\mathrm{ml} / 100 \mathrm{~mL} / \mathrm{min})$} & Median & 170 & $339(59)$ & -50 & -2.9 \\
\hline & IQR & 359 & $140(35)$ & +155 & +6.3 \\
\hline & Local SD & 172 & $79(45)$ & +118 & +2.1 \\
\hline \multirow[t]{3}{*}{ ASL - ATT (s) } & Median & 0.93 & $0.48(0.15)$ & +95 & +3.1 \\
\hline & IQR & 0.99 & $0.43(0.15)$ & +128 & +3.7 \\
\hline & Local SD & 0.45 & $0.20(0.13)$ & +122 & +2.0 \\
\hline 2DPC - Blood flow (ml/min) & NA & 140 & $422(159)$ & -67 & -1.8 \\
\hline \multirow[t]{3}{*}{ DTI - MD $\left(\mathrm{mm}^{2} / \mathbf{s}\right)$} & Median & 1.9 & $2.4(0.1)$ & -19 & -3.6 \\
\hline & IQR & 0.46 & $0.31(0.06)$ & +48 & +2.5 \\
\hline & Local SD & 0.26 & $0.19(0.04)$ & +34 & +1.8 \\
\hline \multirow[t]{3}{*}{ DTI - FA (fraction) } & Median & 0.23 & $0.29(0.03)$ & -20 & -1.9 \\
\hline & IQR & 0.15 & $0.15(0.02)$ & +1 & +0.1 \\
\hline & Local SD & 0.10 & $0.09(0.01)$ & +1 & +0.1 \\
\hline \multirow[t]{3}{*}{ IVIM - D $\left(\mathbf{m m}^{2} / \mathbf{s}\right)$} & Median & 1.7 & $2.1(0.1)$ & -20 & -2.9 \\
\hline & IQR & 0.42 & $0.30(0.07)$ & +41 & +1.8 \\
\hline & Local SD & 0.23 & $0.17(0.03)$ & +31 & +1.5 \\
\hline \multirow[t]{3}{*}{ IVIM - PF (\%) } & Median & $7 \cdot 9$ & $8.8(3.3)$ & -9 & -0.3 \\
\hline & IQR & 10.5 & $10.3(3.1)$ & +1 & o \\
\hline & Local SD & 5.6 & $5.2(1.2)$ & +8 & +0.3 \\
\hline
\end{tabular}

IQR: interquartile range; SD: standard deviation; BOLD: blood oxygenation level-dependent MRI; ASL: arterial spin labelling; AT: arterial transit time; 2DPC: 2D phase contrast; DTI: diffusion tensor imaging; MD: mean diffusivity; FA: fractional anisotropy; IVIM: intravoxel incoherent motion; D: diffusion coefficient; PF: perfusion fraction 

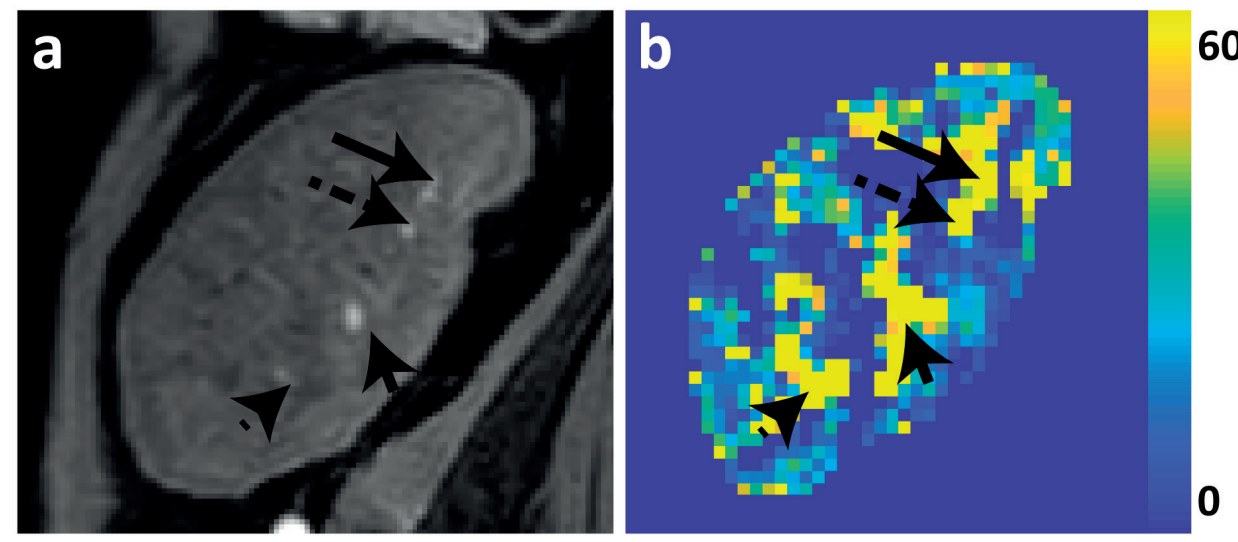

Figure 3. Comparison of anatomical $T_{1}$ weighted Dixon (a) to perfusion map as obtained by ASL (b). In the Dixon, vessels have high signal thanks to inflow of fresh blood. On the perfusion map, vessels can be recognized as areas with very high flow $(\sim 600 \mathrm{~mL} / 100 \mathrm{~mL} /$ min). Because the spatial resolution of the Dixon is much higher compared to the perfusion map (1x1x2 $\mathrm{mm}$ for the Dixon compared to $3 \times 3 \times 6 \mathrm{~mm}$ for the perfusion map), the vessels seem larger and more extensive on the perfusion map. Arrows show the locations of corresponding vessels on the Dixon and on the perfusion map.
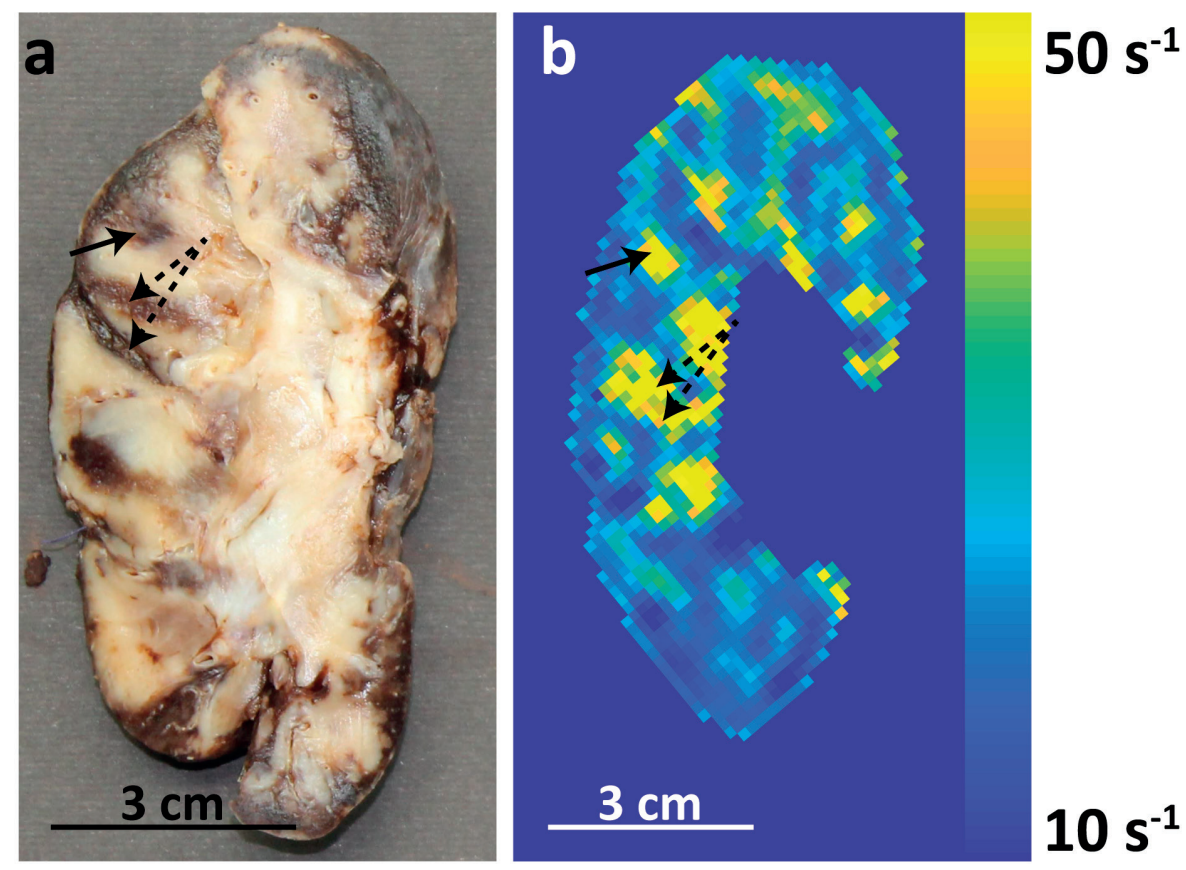

Figure 4. Macroscopic image of the explanted kidney (panel A)shown alongside the corresponding $\mathrm{R}_{2}^{*}$ map (panel $\mathrm{B}$ ). The $\mathrm{R}_{2}{ }^{*}$ map clearly shows the hemorrhages which are denoted by the arrows. The characteristic shape of the hemorrhage at the dashed arrows can also be recognized on the $\mathrm{R}_{2}{ }^{*}$ map 

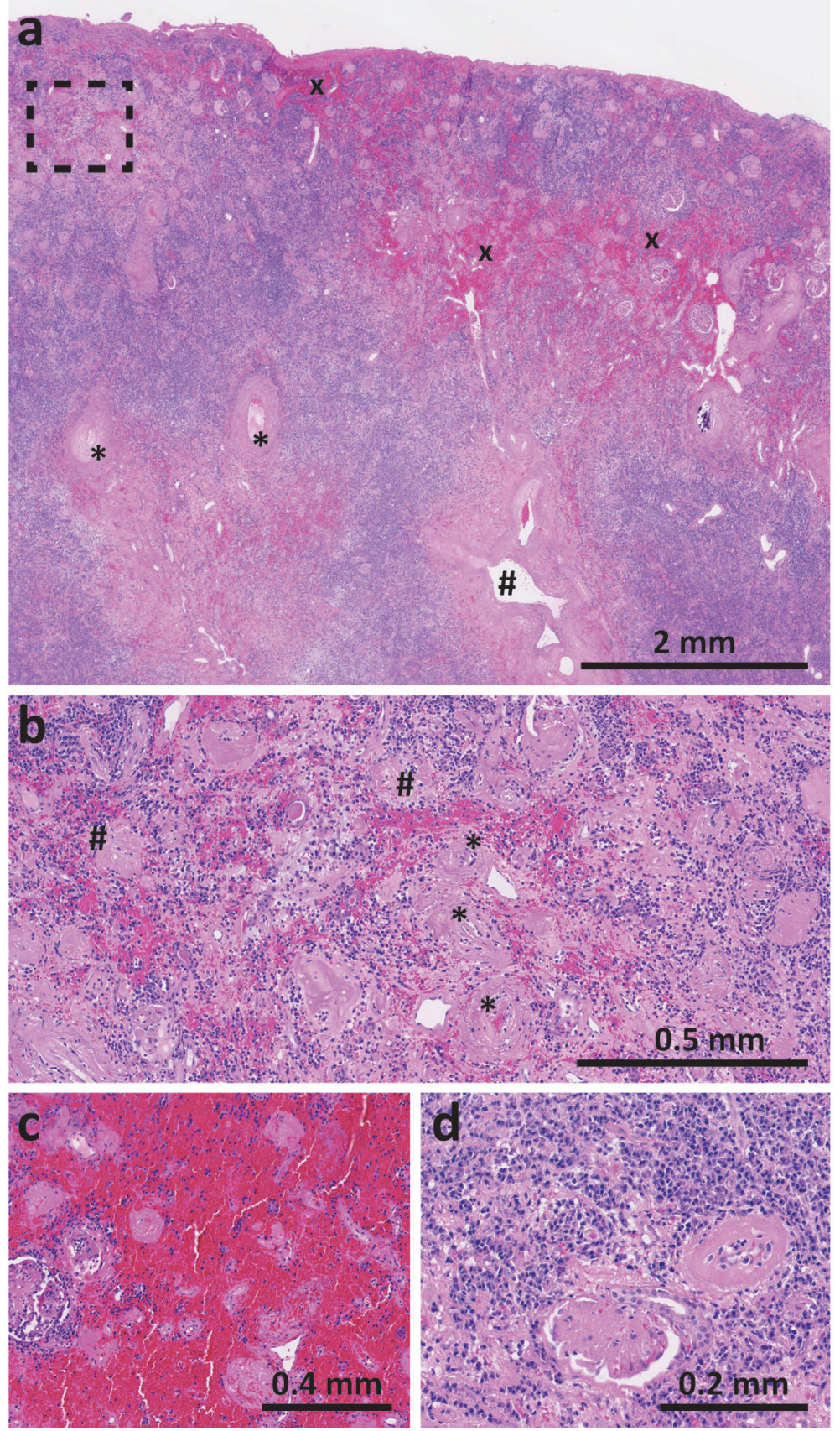

Figure 5. Histology findings of the explanted kidney (all H\&E stain); a) Overview of cortex and medulla. The medulla contains patent interlobar arteries (\#) and arcuate arteries $\left(^{*}\right)$. Regions affected by hemorrhaging are denoted with an $\mathrm{x}$; b) Higher magnification of cortex annotated by the dashed rectangle in A showing completely obliterated interlobular arteries $\left(^{*}\right)$ and globally sclerosed glomeruli (\#); c) Cortical area with extensive interstitial hemorrhage; d) Cortical area with dense interstitial inflammatory infiltrate. 


\section{Histology - Comparison to MRI}

Macroscopically, the explanted kidney was small (10 x 4,5 x 4,5 cm) and multiple hematomas were seen (Figure 4a). Hematomas are expected to be visible at $\mathrm{R}_{2}{ }^{*}$ (BOLD) maps as area's with high $\mathrm{R}_{2}^{*}$. The areas with $\mathrm{R}_{2}{ }^{*}$ close to $5 \mathrm{O} \mathrm{s}^{-1}$ (the cut-off value) seen on the $R_{2}^{*}$ map correlated with the locations of hematomas (Figure $4 \mathrm{a}$ and b).

Microscopic examination revealed extensive microstructural damage as compared to healthy histology (Supplemental Figure 1). Most proximal vasculature, including the interlobar and arcuate arteries in the medulla, was still patent (Figure $5 \mathrm{a}$ ), while the distal vasculature including the interlobular arteries in the cortex were severely obliterated due to concentric intimal fibrosis with infiltration of plasma cells and lymphocytes (Figure 5b). This was consistent with the perfusion maps obtained from ASL, which showed diminished perfusion of the parenchyma with very high perfusion around large vessels (Figure 1).

Throughout the kidney but particularly at the dorsal side of the upper pole and lateral side, extensive areas with interstitial hemorrhages were seen (Figure 5c). Most erythrocytes within these hemorrhages were intact and showed properties consistent with acute hemorrhage, although some scattered areas with hemosiderinladen macrophages were found, indicative of older hemorrhage. The cortex was affected by severe glomerulosclerosis, tubular atrophy and interstitial fibrosis, and contained a dense inflammatory infiltrate consisting mainly of plasma cells (Figure 5d). The medulla displayed a similar severity of inflammation. Although some nonsclerosed glomeruli were present focally, atrophy, inflammation and hemorrhage affected almost all proximal tubules. Those global changes in the renal parenchyma were in line with the MRI findings. These showed increased $\mathrm{T}_{1}$ and restricted diffusion consistent with inflammatory infiltrate and fibrosis and a decreased $\mathrm{T}_{2}$ which is consistent with fibrosis and hemorrhages.

\section{Discussion}

In this study, we aimed to validate multiparametric MRI of a kidney transplant with end-stage disease to whole-kidney histology obtained after explantation. Multiparametric MRI findings were compared to normative values and to histology. Important clinical predictors of graft survival, IF/TA and capillary rarefaction, could be measured with MRI. IF/TA resulted in restriction of diffusion as detected by a decrease in $\mathrm{MD}$ and $\mathrm{D}$ and in an increase in $\mathrm{T}_{1}$. Capillary rarefaction could be identified by reduced parenchymal perfusion, which was detected as a decrease in total perfusion by ASL and 2DPC, as well as by a radical shift in perfusion distribution on ASL perfusion maps. 
The combination of fibrosis and inflammation in the allograft parenchyma was reflected by increased $\mathrm{T}_{1}$ and diffusion restriction ${ }^{(12,13)}$. On the other hand, $\mathrm{T}_{2}$ was decreased, probably due to the severe fibrosis ${ }^{(14,15)}$ and the scattered hemorrhages since dominance of the widespread inflammation would have been expected to result in increase of $\mathrm{T}_{2}^{(16)}$.

Global changes in median $\mathrm{R}_{2}{ }^{*}$ and $\mathrm{R}_{2}$ ' were minimal. However, heterogeneity of $\mathrm{R}_{2}{ }^{*}$ as measured with the local SD was markedly increased (+4.0 SD compared to controls). This probably was caused by the widespread fresh hemorrhages that caused local increases in $R_{2}^{*}$ values. While the hemorrhages also increased $R_{2}^{*}$ and $\mathrm{R}_{2}$ ' on a global level, this was compensated by a decrease in $\mathrm{R}_{2}^{*}$ (and $\mathrm{R}_{2}$ ) due to capillary rarefaction which reduced blood volume and perfusion. Diminished oxygen demand in the virtually nonfunctioning kidney decreased deoxyhemoglobin synthesis as well, decreasing $\mathrm{R}_{2}^{*}{ }^{(17)}$. However, this was compensated by the oxygen demand of active inflammatory cells.

Perfusion of the diseased kidney was less than half of that measured in control subjects as demonstrated by both ASL and 2DPC. The large increase in median ATT indicated that arterial blood supply to the renal parenchyma was heavily delayed. Perfusion maps showed diminished parenchymal perfusion with maintained perfusion in the large vessels. This was consistent with histology showing patent interlobar and arcuate arteries, whereas the most distal interlobular arteries and arterioles were obliterated.

The obliteration of smaller vessels would have been expected to result in a decreased PF as measured with $\mathrm{DWI}^{(5)}$. In repeatability studies in healthy volunteers and patients, large variations in repeat measurements of PF have been observed [5,18-20,9]. The lack of difference in PF in might therefore be attributable to an unreliable measurement, which makes it currently less suitable for diagnostic purposes.

Changes in most MRI measurements in the diseased transplant kidney correlated well with pathological changes seen at macroscopic as well as microscopic analysis of the explanted allograft. Since the diseased allograft had been nonfunctioning for years prior to MRI and explantation, not all results of this study can be simply extrapolated to predict and interpret MRI findings in less damaged transplants. For example, the heterogeneity seen on virtually all MRI measurements was probably caused by scattered hemorrhages, which usually do not occur in earlier stages allograft nephropathy. The histological lesions that are routinely seen in less damaged transplants, such as fibrosis and inflammation, were present in an extreme form that is usually not seen in a functioning graft.However, the changes in $\mathrm{T}_{1}$, diffusion coefficients and perfusion which we found are in line with literature on chronic kidney disease both in native kidneys and transplants and probably do 
reflect the final stage of a continuous process. $\mathrm{T}_{1}$, diffusion coefficients and perfusion measurements are promising non-invasive surrogate markers for severity of fibrosis and IF/TA, inflammation, and capillary rarefaction. However, for clinical diagnostic use of MRI, its lack of specificity remains a problem. The decrease in $\mathrm{T}_{2}$ we found is interesting in this context, since it is expected to change differently in response to inflammation then to fibrosis, but more research in renal disease is needed to confirm this.

A methodological limitation of this study was that the explanted kidney was sectioned at only three levels in the coronal plane (ventral, dorsal and lateral) of the upper- and lower pole. These sections therefore did not exactly match the coronal plane of the MRI scans. This might have led to underestimation of the accuracy by which MRI scans can detect histological abnormalities. During explantation, superficial damage was inflicted to the kidney which might have caused superficial hemorrhaging and consequently might have influenced histology. In addition, the damage to the graft was to an extent usually not seen in healthy grafts. Although our findings are in line with previous studies on MRI in patients with chronic kidney disease, it is unknown how they will translate to less severely damaged grafts. However, if in such an extreme case no changes in MRI parameters could be observed, changes are likely also not observed in less severe cases. Furthermore, the MRI images were compared to images and normative values obtained in healthy volunteers, as opposed to patients with well-functioning transplants which would have been more appropriate, but this was beyond the scope of this study. Kidney transplantation is known to induce physiological and microstructural changes in the graft, which can be measured with MRI, ${ }^{(5)}$ so a comparison to well-functioning grafts might yield slightly different results. In future studies, we aim to make a comparison between ill-functioning and relatively healthy transplant kidneys.

Currently, acquisition time of this multiparametric protocol was approximately one hour, which might be challenging to fit in clinical timeslots. Future studies will most likely determine which acquisitions provide clinically valuable information and which acquisitions do not contribute and can therefore be discarded. This will shorten acquisition time.

In conclusion, this patient gave us the unique opportunity to directly compare MR images obtained shortly before explantation with whole organ histology. Important histological predictors of long term graft survival after kidney transplantation, like IF/TA and capillary rarefaction, could be identified with a combination of multiple functional MRI techniques, which underscores the high potential of the latter for non-invasive acquisition of valuable information for clinical decision making. 


\section{References}

1. Abecassis M, Bartlett ST, Collins AJ et al. (2008) Kidney transplantation as primary therapy for end-stage renal disease: a National Kidney Foundation/Kidney Disease Outcomes Quality Initiative (NKF/KDOQITM) conference. Clin J Am Soc Nephrol 3 (2):471-480. doi:10.2215/CJN.05021107

2. Josephson MA (2011) Monitoring and managing graft health in the kidney transplant recipient. Clin J Am Soc Nephrol 6 (7):1774-1780. doi:10.2215/CJN.01230211

3. Brachemi S, Bollee G (2014) Renal biopsy practice: What is the gold standard? World J Nephrol 3 (4):287-294. doi:10.5527/wjn.v3.i4.287

4. Selby NM, Blankestijn PJ, Boor P et al. (2018) Magnetic resonance imaging biomarkers for chronic kidney disease: a position paper from the European Cooperation in Science and Technology Action PARENCHIMA. Nephrol Dial Transplant 33 (suppl_2):ii4-ii14. doi:10.1093/ndt/gfy152

5. Bane O, Hectors SJ, Gordic S et al. (2020) Multiparametric magnetic resonance imaging shows promising results to assess renal transplant dysfunction with fibrosis. Kidney Int 97 (2):414-420. doi:10.1016/j.kint.2019.09.030

6. Friedli I, Crowe LA, Berchtold L et al. (2016) New Magnetic Resonance Imaging Index for Renal Fibrosis Assessment: A Comparison between Diffusion-Weighted Imaging and T1 Mapping with Histological Validation. Sci Rep 6:30088. doi:10.1038/srep30088

7. Wang W, Yu Y, Wen J et al. (2019) Combination of Functional Magnetic Resonance Imaging and Histopathologic Analysis to Evaluate Interstitial Fibrosis in Kidney Allografts. Clin J Am Soc Nephrol 14 (9):1372-1380. doi:10.2215/cjn.00020119

8. Cutajar M, Thomas DL, Hales PW et al. (2014) Comparison of ASL and DCE MRI for the non-invasive measurement of renal blood flow: quantification and reproducibility. Eur Radiol 24 (6):1300-1308. doi:10.1007/so0330-014-3130-o

9. De Boer A, Harteveld AA, Stemkens B et al. (2020) Multi-Parametric Renal MRI - an Intra-Subject Test-Retest Repeatability Study.

10. Cosio FG, El Ters M, Cornell LD, Schinstock CA, Stegall MD (2016) Changing Kidney Allograft Histology Early Posttransplant: Prognostic Implications of 1-Year Protocol Biopsies. American journal of transplantation : official journal of the American Society of Transplantation and the American Society of Transplant Surgeons 16 (1):194-203. doi:10.1111/ajt.13423

11. Steegh FMEG, Gelens MACJ, Nieman FHM et al. (2011) Early Loss of Peritubular Capillaries after Kidney Transplantation. Journal of the American Society of Nephrology 22 (6):1024. doi:10.1681/ASN.2010050531

12. Caroli A, Schneider M, Friedli I et al. (2018) Diffusion-weighted magnetic resonance imaging to assess diffuse renal pathology: a systematic review and statement paper. Nephrol Dial Transplant 33 (suppl_2):ii29-ii40. doi:10.1093/ndt/gfy163 
13. Wolf M, de Boer A, Sharma K et al. (2018) Magnetic resonance imaging T1- and T2mapping to assess renal structure and function: a systematic review and statement paper. Nephrol Dial Transplant 33 (suppl_2):ii41-ii5o. doi:10.1093/ndt/gfy198

14. Di Cesare E (2001) MRI of the cardiomyopathies. Eur J Radiol 38 (3):179-184. doi:10.1016/ s0720-048x(01)00311-4

15. Matsumoto S, Mori H, Miyake $\mathrm{H}$ et al. (1998) MRI signal characteristics of progressive massive fibrosis in silicosis. Clin Radiol 53 (7):510-514. doi:10.1016/sooo99260(98)80171-2

16. Kim PK, Hong YJ, Im DJ et al. (2017) Myocardial T1 and T2 Mapping: Techniques and Clinical Applications. Korean J Radiol 18 (1):113-131. doi:10.3348/kjr.2017.18.1.113

17. Niendorf T, Pohlmann A, Arakelyan K et al. (2015) How bold is blood oxygenation leveldependent (BOLD) magnetic resonance imaging of the kidney? Opportunities, challenges and future directions. Acta Physiol (Oxf) 213 (1):19-38. doi:10.1111/apha.12393

18. Cox EF, Buchanan CE, Bradley CR et al. (2017) Multiparametric Renal Magnetic Resonance Imaging: Validation, Interventions, and Alterations in Chronic Kidney Disease. Front Physiol 8:696. doi:10.3389/fphys.2017.00696

19. Bane O, Wagner M, Zhang JL et al. (2016) Assessment of renal function using intravoxel incoherent motion diffusion-weighted imaging and dynamic contrast-enhanced MRI. J Magn Reson Imaging 44 (2):317-326. doi:10.1002/jmri.25171

20. Pan J, Zhang H, Man F et al. (2018) Measurement and scan reproducibility of parameters of intravoxel incoherent motion in renal tumor and normal renal parenchyma: a preliminary research at 3.0 T MR. Abdom Radiol (NY) 43 (7):1739-1748. doi:10.1007/ soo261-017-1361-7 


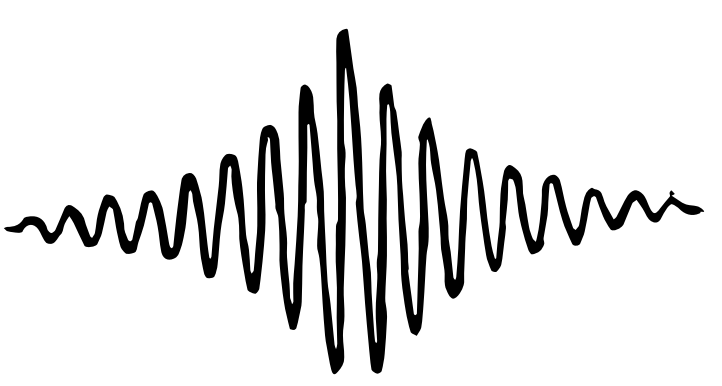




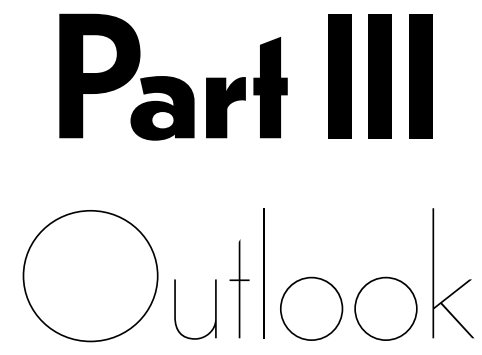





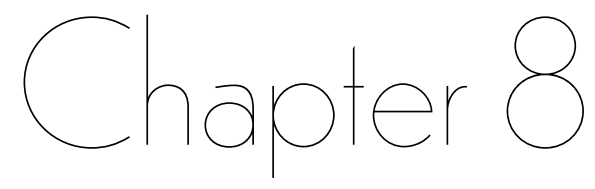

\section{T Renal MRI: Challenges and Promises}

$$
\frac{d}{d t}\left(\begin{array}{l}
M_{x} \\
M_{y} \\
M_{2}
\end{array}\right)=\left(\begin{array}{ccc}
-\frac{1}{T_{2}} & \gamma B_{2} & -\gamma B_{y} \\
-\gamma B_{2} & -\frac{1}{T_{2}} & \gamma B_{x} \\
\gamma B_{y} & -\gamma B_{x} & -\frac{1}{T_{1}}
\end{array}\right)\left(\begin{array}{c}
M_{x} \\
M_{y} \\
M_{2}
\end{array}\right)+\left(\begin{array}{c}
0 \\
0 \\
\frac{M_{0}}{T_{1}}
\end{array}\right)
$$

\section{Author contributions}

de Boer A, Hoogduin JM, Blankestijn PJ, Li X, Luijten PR, Metzger GJ, Raaijmakers AJ, Umutlu L, Visser F, Leiner T

$$
\text { Published in }
$$

Magn Reson Mater Phy 2016; doi.org/10.1007/s10334-016-0538-3 


\section{Abstract}

The progression to $7 \mathrm{~T}$ MRI yields promises of substantial increase in signal-to-noise (SNR) ratio. This increase can be traded off to increase image spatial resolution or to decrease acquisition time. However, renal $7 \mathrm{~T}$ MRI remains challenging due to inhomogeneity of the radiofrequency field and due to specific absorption rate (SAR) constraints. A number of studies has been published in the field of renal $7 \mathrm{~T}$ imaging. While the focus initially was on anatomic imaging and renal MR angiography, later studies have explored renal functional imaging. Although anatomic imaging remains somewhat limited by inhomogeneous excitation and SAR constraints, functional imaging results are promising. The increased SNR at $7 \mathrm{~T}$ has been particularly advantageous for blood oxygen level dependent and arterial spin labelling MRI, as well as sodium MR imaging, thanks to changes in field-strength dependent magnetic properties. Here we provide an overview of the currently available literature on renal 7T MRI. In addition, we provide a brief overview of challenges and opportunities in renal $7 \mathrm{~T}$ MR imaging 


\section{Introduction}

Since publication of the first in vivo MRI study of the human brain at $8 \mathrm{~T}^{(1)}$, imaging at fields equal to or higher than $7 \mathrm{~T}$ has been mostly limited to neuroimaging and, to a lesser degree, extremities ${ }^{(2)}$. The first body images were presented in $2007^{(3)}$ showing the potential of prostate imaging at $7 \mathrm{~T}$. Driven by the ambition to increase spatial and temporal resolution and to improve contrast, $7 \mathrm{~T}$ body MRI emerged further, and the first abdominal images were published in $2009^{(4)}$. Nowadays 7 T MRI even is used in cardiac imaging, one of the most challenging fields in MR imaging ${ }^{(5,6)}$.

The increase in signal-to-noise ratio (SNR) at higher field strengths can be used to increase spatial resolution or to decrease imaging time by using under sampling strategies such as parallel imaging or compressed sensing. In addition to the increased SNR several other features need to be considered when imaging at high fields. First, there is the increased water-fat shift due to the higher proton Larmor frequency, which can result in larger chemical shift artefacts. However, the larger water-fat shift can potentially be utilized to improve fat suppression. The second issue that needs to be considered is the increased severity of susceptibility effects at $7 \mathrm{~T}$, resulting in a decrease in $\mathrm{T}_{2}{ }^{*}$ relaxation times ${ }^{(7)}$. Just as with the increase in water-fat shift, this has both advantages and drawbacks. In imaging techniques utilizing susceptibility differences, like blood oxygen level dependent (BOLD) MRI or dynamic susceptibility contrast perfusion imaging, an increase in signal can be achieved. However, increased susceptibility effects may also give rise to larger geometric distortions and/or signal loss. Third, relaxation times change at increasing field strengths. In the kidney, this results in an increase in $\mathrm{T}_{1}$ relaxation time and to a lesser degree a decrease in $\mathrm{T}_{2}$ relaxation times ${ }^{(8)}$. For standard paramagnetic contrast agents, the $R_{1}$ relaxivity remains similar or decreases slightly ${ }^{(9-1)}$ while $R_{2}{ }^{*}$ relaxivity increases dramatically in inhomogeneous compartments such as blood ${ }^{(10)}$. To date, standard recommended doses of contrast have been administered at $7 \mathrm{~T}$ for providing the desired $\mathrm{T}_{1}$ enhancement, however optimal dosing has not yet been investigated. Taking advantage of the increased $\mathrm{T}_{1}$ at $7 \mathrm{~T}$ along with attempts to minimize $\mathrm{R}_{2}{ }^{*}$ effects allow for improved performance when using lower doses while simultaneously addressing concerns related to long term effects of contrast agent administration. The increase in $\mathrm{T}_{2}{ }^{*}$ signal attenuation is also of particular interest in dynamic contrast enhanced (DCE) MRI studies, where contrast agent concentrations are calculated for the quantification of pharmacokinetic parameters or flow. Without correction for $\mathrm{T}_{2}{ }^{*}$ weighting, the increased $\mathrm{T}_{2}{ }^{*}$ weighting could introduce large errors in quantitative results ${ }^{(10)}$. Fourth, the increase in Larmor frequency at high field strengths implies a decrease in radiofrequency (RF) wavelength, which is about $12 \mathrm{~cm}$ in tissue at $7 \mathrm{~T}$ - shorter than the typical diameter of the human torso. This results in RF interference patterns creating inhomogeneous excitation and possibly 
areas devoid of signals where interference is destructive (4). Fifth, the global specific absorption rate (SAR), a measure for energy absorbed by the body per unit of time, increases with the square of the electric component of the electromagnetic field ${ }^{(12)}$. Since SAR is limited to maintain temperature rises in the body below $1^{\circ} \mathrm{C}$, this imposes restrictions on the sequences that can be used ${ }^{(12)}$. At $7 \mathrm{~T}$, the large $\mathrm{RF}$ inhomogeneity results in potential increases in local SAR, and even more importantly local heating, which significantly impacts the types of acquisition methods, RF pulses and sequence timings that can be used ${ }^{(13,14)}$.

Multiple groups have worked on these opportunities and challenges, and $7 \mathrm{~T}$ MRI is increasingly used for abdominal MRI, including renal imaging. This review aims to provide an overview of the work currently published on renal 7T MRI in humans from the perspective of relevant renal anatomy, physiology and current clinical practice. In the second part, challenges in renal imaging at $7 \mathrm{~T}$ will be addressed and a short overview of possible solutions will be provided.

\section{Currently Published Work on Renal 7T MRI}

\section{Anatomical Imaging}

The first to investigate feasibility of renal MRI at $7 \mathrm{~T}$ were Umutlu et al. ${ }^{(15)}$. Of the $\mathrm{T}_{1}$ weighted sequences used, 2D spoiled gradient echo (2D FLASH, Figure 1) and 2D inand opposed phase gradient echo performed best in depicting the renal structures. Other $\mathrm{T}_{1}$ weighted images showing better contrast between cortex and medulla were acquired by Metzger et al. ${ }^{(14)}$ (Figure 2). Hoogduin et al. ${ }^{(16)}$ have aimed to extend the work of Umutlu ${ }^{(15)}$ and Metzger et al. ${ }^{(14)}$ by using a multi-echo turbo field echo sequence with Dixon reconstruction for fat suppression (Figure 3). Initial results indicate that good fat suppression can be obtained, although contrast between medulla and cortex is somewhat less than in the images acquired by Metzger et al. As clearly seen in Figure 1, 2 and 3, arteries were hyperintense on $\mathrm{T}_{1}$ weighted sequences. This can be explained by inflow effects due to the absence of saturation of blood entering from regions outside the excitation area ${ }^{(17)}$.

Umutlu et al. ${ }^{(15)}$ performed $\mathrm{T}_{2}$ weighted imaging, but the images were heavily impaired by artefacts due to B1 field inhomogeneity and SAR limitations (Figure 4). $T_{2}$ weighted imaging also was performed by Hoogduin et al. ${ }^{(16)}$ using a turbo spin echo sequence, yielding good quality images with minor artefacts (Figure 5). To keep SAR within limits, a long TR ( $16000 \mathrm{~ms}$ ) and SENSE factor of 5 had to be used. In Tables 1 and 2 parameters of the $\mathrm{T}_{1}$ and $\mathrm{T}_{2}$ weighted sequences are provided, respectively. 

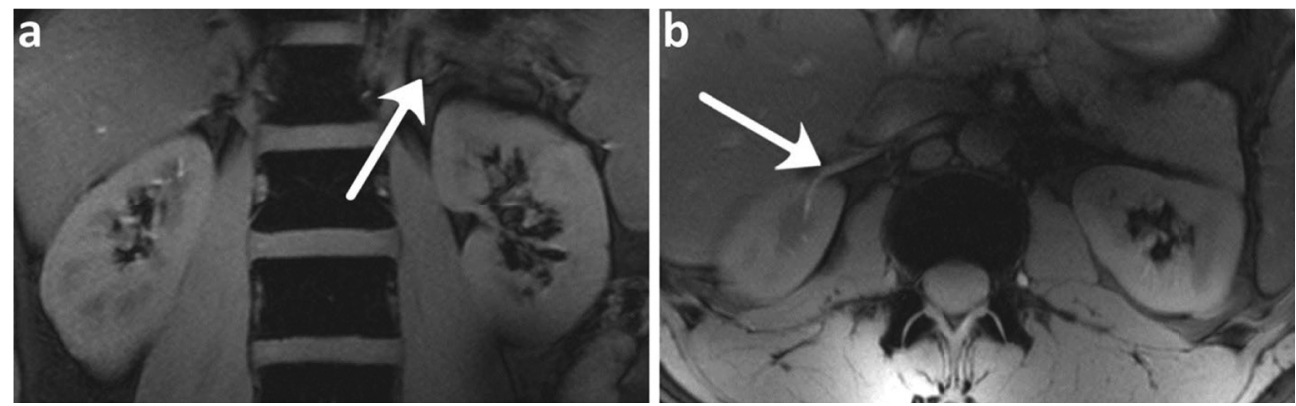

Figure 1. Coronal $T_{1} w_{2} D$ FLASH images acquired $\mathbf{a}$ arrow: adrenal gland $\mathbf{b}$ arrow: renal vasculature with high signal intensity. (Umutlu et al., unpublished results)
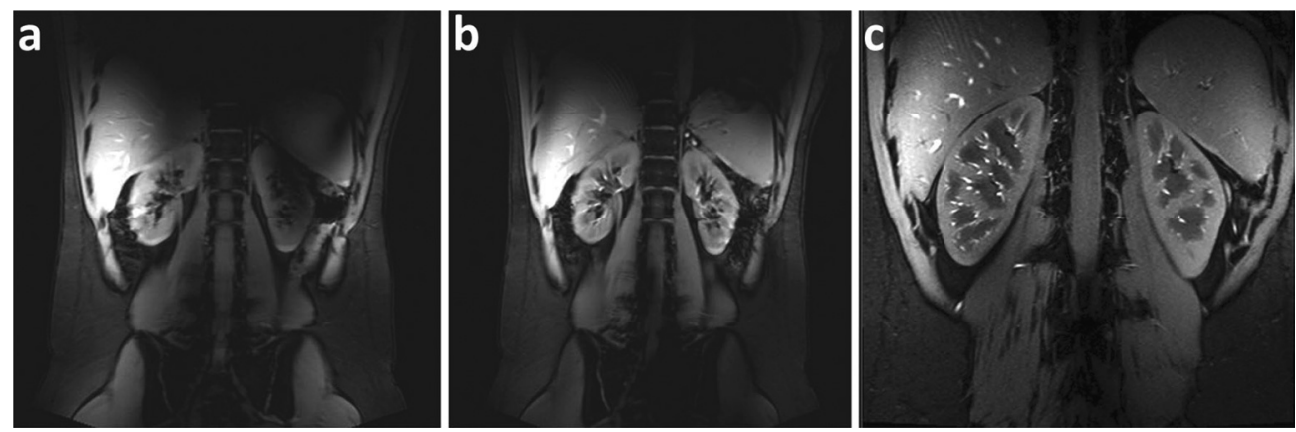

Figure 2. Coronal $\mathrm{T}_{1} \mathrm{w}$ gradient echo images a without $\mathrm{B}_{1}^{+}$shimming, $\mathbf{b}$ with local $\mathrm{B}_{1}^{+}$ shimming and $\mathbf{c}$ a high resolution version of b (FOV $240 \mathrm{~mm}$, slice thickness $2.2 \mathrm{~mm}$, remaining parameters the same). (Metzger et al. ${ }^{(14)}$ )
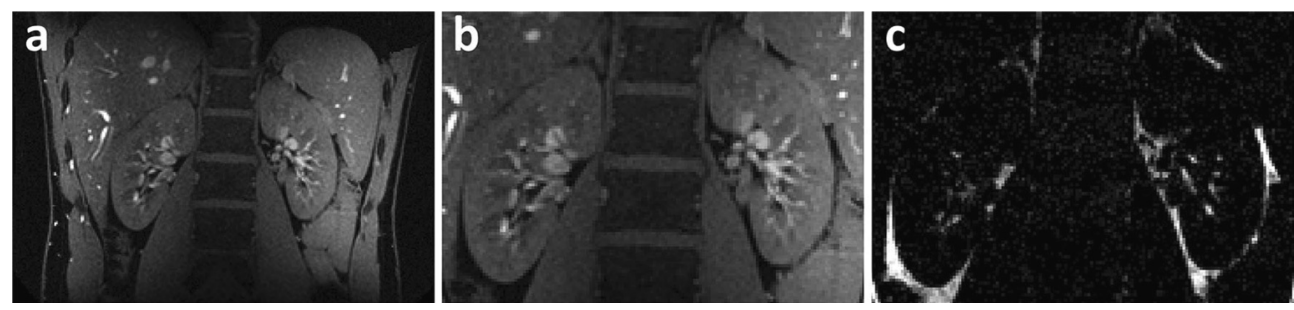

Figure 3. Anatomical images acquired coronal TFE images with Dixon reconstruction; a water image; b zoom of a and c fat image. (Hoogduin et al. ${ }^{(16)}$ )
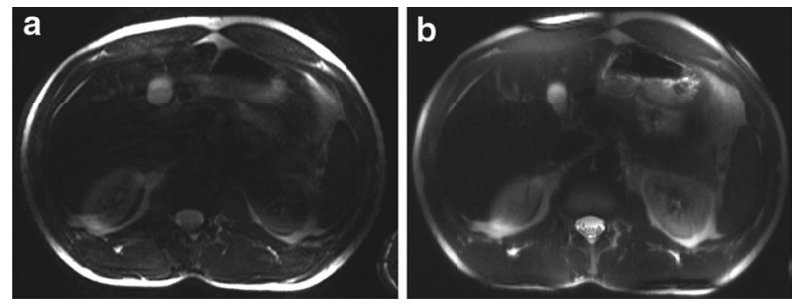

Figure 4. Axial $\mathrm{T}_{2} \mathrm{w}$ TSE images acquired in two subjects a strongly impaired and $\mathbf{b}$ with medium image quality. (Umutlu et al., unpublished results). 

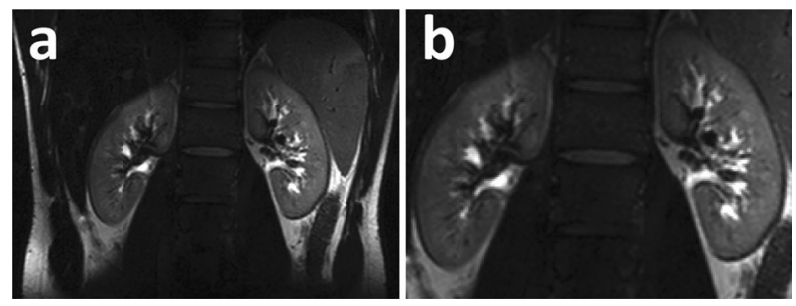

Figure 5. a Coronal $\mathrm{T}_{2} \mathrm{w}$ TSE images; b zoom of a. (Hoogduin et al. ${ }^{(16)}$ )

Table 1. Imaging parameters for the $T_{1}$ weighted anatomical sequences.

\begin{tabular}{l|cccc}
\hline Sequence & 2D FLASH $^{(15,18)}$ & 3D FLASH $^{(\mathbf{1 5}, 18)}$ & T1w GE $^{(14)}$ & T1w ME TFE $^{(\mathbf{1 6})}$ \\
\hline Slice orientation & coronal & coronal & coronal \& axial & coronal \\
TR/TE [ms] & $130 / 3.57$ & $2.9 / 1.02$ & $150 / 3.7$ & $5.0 / 2.1 ; 2.7 ; 3.3$ \\
Nominal flip angle $\left[^{\circ}{ }^{\circ}\right.$ & 70 & 10 & 60 & 10 \\
FOV [mm] & 400 & 400 & 450 & 370 \\
Voxel volume [mm] & $0.8 \times 0.8 \times 2.0$ & $1.3 \times 1.3 \times 1.6$ & $1.9 \times 1.2 \times 5$ & $1.49 \times 1.49 \times 3.00$ \\
Slices & 13 & 128 & 14 & 12 \\
Acquisition time [s] & $31^{\mathrm{a}}$ & $27^{\mathrm{a}, \mathrm{b}}$ & $18^{\mathrm{a}}$ & $44^{\mathrm{c}}$ \\
Parallel imaging & GRAPPA 2 & GRAPPA 2 & GRAPPA 2 & SENSE 2 \\
\hline
\end{tabular}

a: equals breath hold length; b: one slice per breath hold ( $3.6 \mathrm{~s})$; b: Using $66 \%$ over contiguous slices and 6/8 partial Fourier in phase and slice direction; c: One slice per breath hold (3.6 s); ME: multi-echo; TFE: turbo field echo.

Table 2. Imaging parameters for the $\mathrm{T}_{2}$ weighted anatomical sequences.

\begin{tabular}{l|ll}
\hline Sequence & T2w TSE ${ }^{(15,18)}$ & T2w TSE $^{(16)}$ \\
\hline Slice orientation & axial & coronal \\
TR/TE [ms] & $3060 / 99$ & $16000 / 187$ \\
Nominal flip angle $\left[^{\circ}\right]^{-120}$ & $150 \times 240$ & 125 \\
FOV [mm] & $1.4 \times 1.4 \times 5.5$ & $375 \times 518$ \\
Voxel volume [mm] & 16 & $1.4 \times 1.6 \times 5.0$ \\
Slices & $34^{\mathrm{a}}$ & 5 \\
Acquisition time [s] & GRAPPA 2 & $80^{\mathrm{b}}$ \\
Parallel imaging & & SENSE $_{5}$ \\
\hline
\end{tabular}

a: equals breath hold time; b: respiratory triggering.

The effect of contrast administration both on renal vasculature and corticomedullary differentiation was investigated by Umutlu et al. ${ }^{(18)}$. They acquired 3D FLASH images before and 20, 70 and 120 seconds after contrast administration. Contrast administered in a dose of $0.1 \mathrm{mmol} / \mathrm{kg}$ resulted in a slight improvement in contrast between cortex and medulla and better depiction of the renal arteries on $3 \mathrm{D}$ FLASH images with respect to 2D FLASH, but only in the image acquired during the arterial phase (20 s post-contrast). 


\section{$T_{1}$ and $T_{2}$ Measurements}

Since proton relaxation times depend on field strength, measurements of kidney $\mathrm{T}_{1}$ and $\mathrm{T}_{2}$ are desirable to optimize scan protocols. Thus far, only one group performed measurements of relaxation times ${ }^{(8)}$. Measurements were performed in eleven healthy volunteers and five subjects were scanned twice to evaluate reproducibility. Values were compared to measurements on $3 \mathrm{~T}$ using the same sequences with similar parameters. To assure a uniform inversion, $\mathrm{B}_{1}^{+}$shimming was applied only for one kidney, resulting in a coefficient of variation of $\mathrm{B}_{1}^{+}$inhomogeneity of $8 \%$. For $\mathrm{T}_{1}$ measurements, a single-shot fast spin echo (ss-FSE) was used with adiabatic inversion pulses at six different inversion times. For $\mathrm{T}_{2}$ measurement, a Carr-PurcellMeiboom-Gill (CPMG) refocusing pulse train was inserted as a preparation module in between excitation and readout to minimize signal loss due to diffusion. Within this preparation module on each side of the refocusing pulses spoiling gradients were applied to prevent the formation of stimulated echoes. Measurements consisting of a single transversal slide could be performed in a single breath hold. $\mathrm{T}_{1}$ and $\mathrm{T}_{2}$ maps are shown in Figure 6 and mean relaxation times are given in Table $3 . \mathrm{T}_{2}$ values measured for comparison at $3 \mathrm{~T}$ were significantly higher than values known in the literature, possibly due to the pulse sequence which was designed to reduce signal loss due to diffusion by insertion of a CPMG refocusing pulse train.
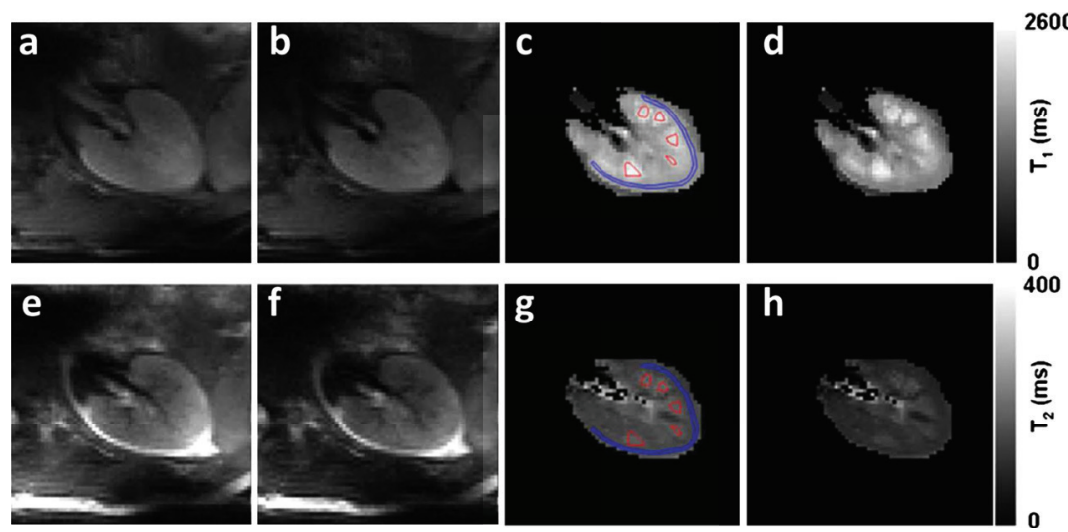

Figure 6. a and $\mathbf{b}$ ss-FSE images for $\mathrm{T}_{1}$ measurement with inversion time 100 and $150 \mathrm{~ms}$, respectively; $\mathbf{c}$ and $\mathbf{d} \mathrm{T}_{1}$ maps with and without ROIs for $\mathrm{T}_{1}$ estimation; $\mathbf{e}$ and $\mathbf{f}$ ss-FSE images for $\mathrm{T}_{2}$ measurement with effective echo time 20 and $40 \mathrm{~ms}$; $\mathbf{g}$ and $\mathbf{h} \mathrm{T}_{1}$ maps with and without ROIs for $\mathrm{T}_{1}$ estimation (all ss-FSE images were acquired at six different inversion times or effective echo times to minimize short-term SAR) (Li et al. ${ }^{(8)}$ )

Table 3. $\mathrm{T}_{1}$ and $\mathrm{T}_{2}$ values on 3 and $7 \mathrm{~T}(8)$.

\begin{tabular}{l|c|c|c|c}
\hline & \multicolumn{2}{|c}{ Cortex } & \multicolumn{2}{c}{ Medulla } \\
& $\mathbf{T}_{\mathbf{1}}$ & $\mathbf{T}_{\mathbf{2}}$ & $\mathbf{T}_{\mathbf{1}}$ & $\mathbf{T}_{\mathbf{2}}$ \\
\hline $\mathbf{3 T}$ & $1261 \pm 68$ & $121 \pm 5$ & $1676 \pm 94$ & $138 \pm 7$ \\
$7 \mathbf{T}$ & $1668 \pm 46$ & $109 \pm 6$ & $2095 \pm 52$ & $125 \pm 5$ \\
\hline
\end{tabular}




\section{Angiography}

In two separate publications, Umutlu et al. described the possibility of $7 \mathrm{~T}$ renal MR angiography using both native 2D gradient echo time of flight (TOF) MRA as well as using contrast-enhanced techniques ${ }^{(19,20)}$. In both studies, image quality of angiographic sequences was assessed on a 5-point scale with 1 denoting nondiagnostic and 5 denoting excellent delineation. To image renal vasculature, gradient echo TOF MRA was compared to $\mathrm{T}_{1}$ weighted 2D and 3D FLASH, where the vasculature was hyperintense as observed earlier ${ }^{(19)}$. While 2D FLASH suffered from artefacts (mainly inflow effects and motion) and low SNR and CNR, both 3D FLASH and TOF MRA performed well. In 3D FLASH moderate background suppression led to a low contrast to noise ratio (CNR) with respect to the psoas major muscle, despite good SNR. The authors concluded that TOF MRA was capable of superior delineation of the aorta and left and right renal arteries compared to both $2 \mathrm{D}$ and $3 \mathrm{D}$ FLASH and superior CNR (Figure 7), although some venous overlay was present due to insufficient background suppression. For TOF MRA mean SNR was 54 and mean quality score was 4.7 out of 5 .
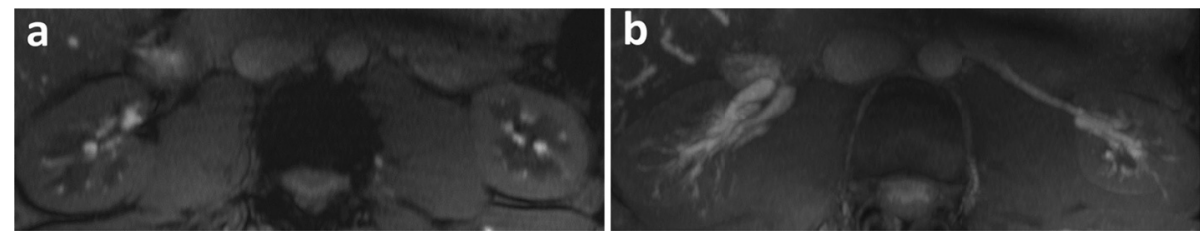

Figure 7. a TOF MRA and $\mathbf{b}$ maximum intensity projection of TOF MRA acquired (Umutlu et al., unpublished results).

Umutlu et al. also compared contrast enhanced (CE) MRA to unenhanced 3D FLASH MRI ${ }^{(20)}$. Contrast enhancement mainly improved delineation of the right renal artery (Figure 8), while improvement was less pronounced in the left renal artery and the aorta. CNR improved about two fold for all arteries, ranging from 45 to 60 , with SNR ranging from 90 to 105 . Mean quality score of CE 3 D FLASH was 4.8. However, measurements were only performed in healthy volunteers, so no information is available on the grading of renal artery stenosis on $7 \mathrm{~T}$.
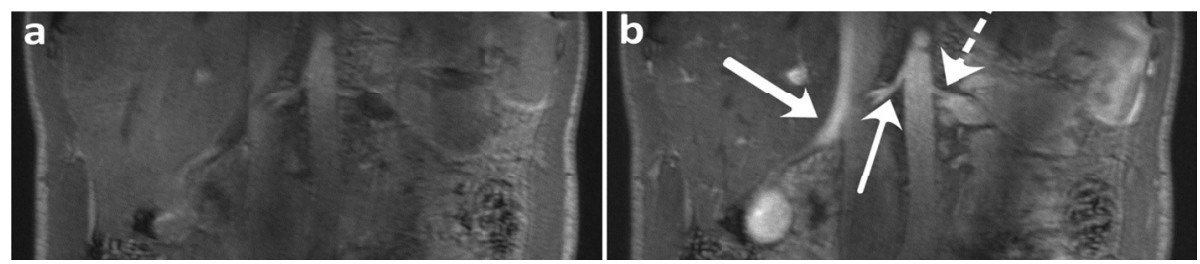

Figure 8. a Unenhanced and b CE 3D FLASH. Wide arrow: right renal vein; slim arrow: right renal artery; dashed arrow: left renal artery. (Umutlu et al., unpublished results). 
Metzger et al. also explored the possibility of renal artery imaging at $7 \mathrm{~T}$ using non-CE MRA with a 3D turbo FLASH sequence ${ }^{(14)}$. To minimize background signals, a frequency selective saturation pulse was used to reduce lipid signals and a slab selective adiabatic inversion was used to null the renal parenchyma. Different $\mathrm{B}_{1}^{+}$shimming solutions were used for the adiabatic inversion and the other $\mathrm{RF}$ pulses in the sequence (i.e. the fat saturation and excitation). The inversion pulse required a much higher $\mathrm{B}_{1}^{+}$thus benefitted from an efficiency solution optimized over the kidneys while the excitation and lipid suppression benefitted from more homogeneous $\mathrm{B}_{1}^{+}$over a larger region of interest. Dynamically applying these shim solutions within a sequence addressed the fact there is an implicit trade-off between RF homogeneity and efficiency preventing both conditions from being accomplished simultaneously. In Figure 9, maximum intensity projections (MIPs) are shown in multiple subjects in which this acquisition strategy was employed. Note the excellent depiction of small vessels which are not obscured by the renal parenchyma as is often the case when using intravenously injected contrast agents. Imaging parameters for all sequences are given in table 4 .

Table 4. Imaging parameters for MR angiography sequences.

\begin{tabular}{l|ccc}
\hline Sequence & CE $^{\text {3D FLASH }}{ }^{(20)}$ & 2D GE TOF $^{(19)}$ & 3D Turbo FLASH $^{(14)}$ \\
\hline Slice orientation & coronal & transverse & coronal \& axial \\
TR/TE [ms] & $2.98 / 0.97$ & $17 / 4.70$ & $3.8 / 1.76$ \\
Nominal flip angle $\left[{ }^{\circ}\right.$ ] & 25 & 60 & 8 \\
FOV [mm] & 400 & $250 \times 188$ & $300-380$ \\
Voxel volume [mm] & $1.5 \times 1.0 \times 1.0$ & $1.0 \times 2.0 \times 2.5$ & $1.1-1.2 \times 1.1-1.2 \times 1.0-1.3$ \\
Slices & $\mathrm{NR}$ & 20 & 72 \\
Acquisition time & $20 \mathrm{~s}^{\mathrm{a}}$ & $33 \mathrm{~s}^{\mathrm{a}}$ & 9 min $^{\mathrm{b}}$ \\
Parallel imaging & GRAPPA 2 & GRAPPA 2 & GRAPPA 2 \\
Saturation & Fat saturation & Fat Saturation & Fat saturation \& Adiabatic \\
& & & inversion \\
\hline
\end{tabular}

a: equals breath hold length; b: respiratory triggering; GE: gradient echo; NR: not reported. 

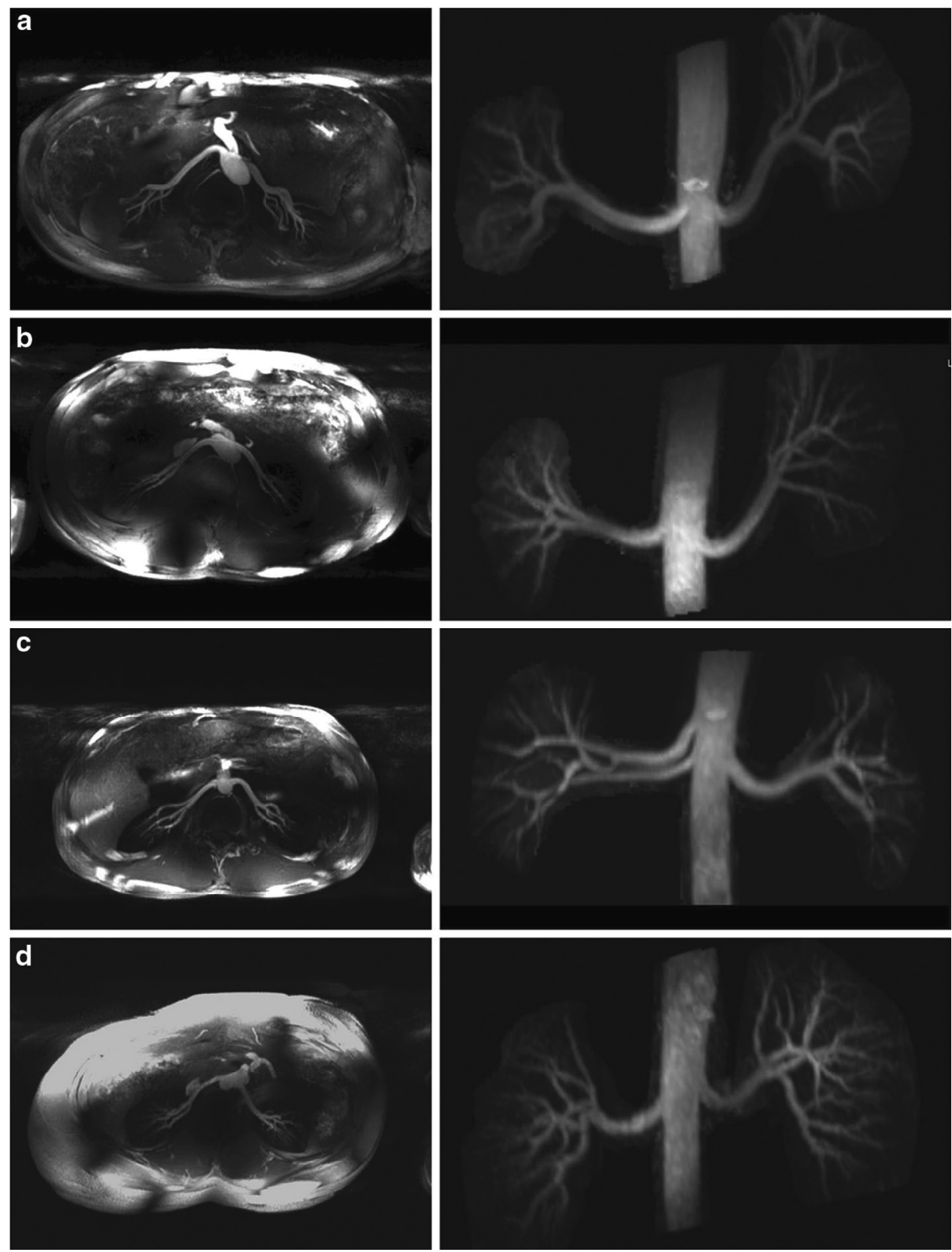

Figure 9. Axial (left) and cropped coronal (right) MIPs from multiple volunteers with different shimming strategies: a phase only homogeneity shim both for saturation and conventional pulses; $\mathbf{b}$ efficiency shim for saturation pulse; $\mathbf{c}$ and $\mathbf{d}$ trade-off solution for saturation pulse, magnitude and phase homogeneous shim for conventional pulses. (Metzger et al. ${ }^{(14)}$ ) 


\section{Functional Imaging}

BOLD MRI

BOLD MRI relies on the difference between deoxygenated and oxygenated haemoglobin, the first being paramagnetic while the second is diamagnetic. Three groups performed renal BOLD MRI at $7 \mathrm{~T}$. Brinkmann et al. ${ }^{(21)}$ performed BOLD imaging using a multi-echo 2D FLASH sequence during water loading both on 3 and $7 \mathrm{~T}$. The decrease in medullary $\mathrm{R}_{2}{ }^{*}$ value during water loading ( $23 \%$ at $3 \mathrm{~T}$ ) was more pronounced on $7 \mathrm{~T}$, where a decrease of $33 \%$ was measured. In addition, the $\mathrm{R}_{2}{ }^{*}$ values were higher at 7T: $90 \mathrm{~s}-1$ versus $29 \mathrm{~s}^{-1}$ on $3 \mathrm{~T}$ in the medulla and $69 \mathrm{~s}^{-1}$ versus $25 \mathrm{~s}^{-1}$ for renal cortex. Hoogduin et al. found similar results using a multi-echo gradient echo sequence. To derive $\mathrm{R}_{2}{ }^{*}$ values for cortex and medulla, they used a compartmental analysis as proposed by Ebrahimi et al. ${ }^{(22)}$. Here, a Gaussian function representing the cortex and a gamma function for the medulla are fitted to the histogram of the data (Figure 10). In comparison with data measured earlier at $3 \mathrm{~T}$ in the same centre ${ }^{(23)}$, at $7 \mathrm{~T}$ a distinct peak for the medulla was visible on the histogram (Figure 10c). $\mathrm{R}_{2}^{*}$ values of 66 and $41 \mathrm{~Hz}$ for the medulla and cortex were measured, respectively. While the $\mathrm{R}_{2}^{*}$ ratio between medulla and cortex in the study by Hoogduin et al. was nearly constant, 1.54 on $3 \mathrm{~T}$ versus 1.60 on $7 \mathrm{~T}$, Brinkmann et al. measured slightly different ratios: 1.16 on $3 \mathrm{~T}$ versus 1.30 on $7 \mathrm{~T}$. Li et al. also performed $\mathrm{T}_{2}{ }^{*}$ mapping, but they did not publish quantitative results ${ }^{(8)}$. Scan parameters are provided in Table 5 .

Table 5. Imaging parameters for BOLD MRI sequences. ME: multi-echo.

\begin{tabular}{l|cc}
\hline Sequence & CE 3D FLASH ${ }^{\text {(2o) }}$ & 2D GE TOF ${ }^{\text {(19) }}$ \\
\hline Slice orientation & coronal & coronal \\
TR [ms] & 96 & 102 \\
Number of echoes & 10 & 20 \\
TE [ms] & $2 ; 6.6 ; \ldots ; 29.1$ & $4.93 ; 9.86 ; \ldots ; 98.6$ \\
FOV [mm] & 256 & $375 \times 381$ \\
Voxel volume [mm] & $1.0 \times 1.0 \times 6$ & $1.5 \times 1.5 \times 5$ \\
Slices & 3 & 3 \\
Acquisition time [s] & 24 & $47^{\mathrm{a}}$ \\
Parallel imaging & $\mathrm{NR}$ & SENSE 2 \\
\hline
\end{tabular}

a: one slice per breath hold (16 s); NR: not reported. 

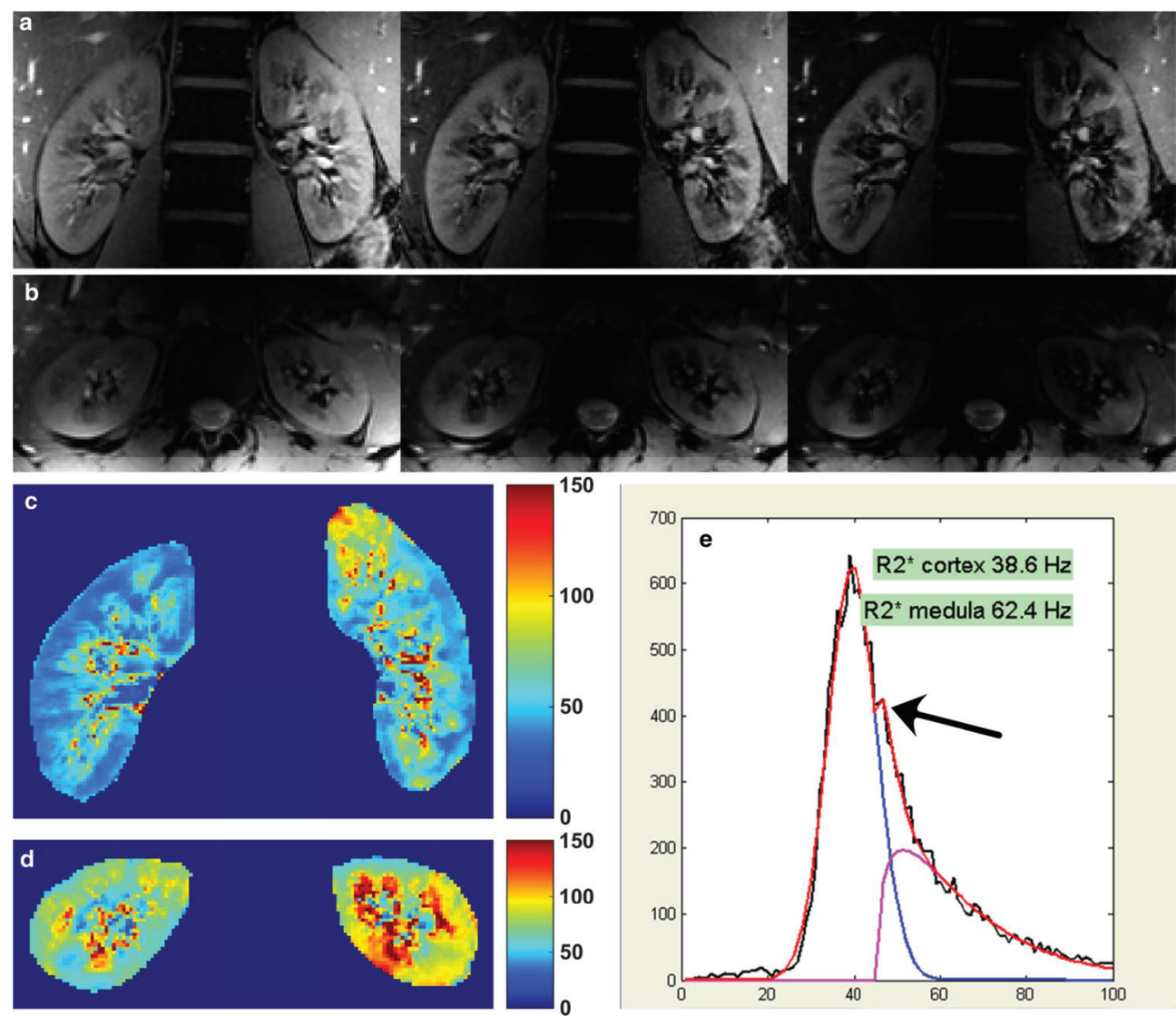

Figure 10. $\mathrm{T}_{2}{ }^{*}$ weighted images: a coronal images (echo times 4.9, 9.8 and $14.7 \mathrm{~ms}$ ); b transversal image (echo times 4.9, 9.8 and $14.7 \mathrm{~ms}$ ); c and $\mathbf{d}$ corresponding $\mathrm{R}_{2}^{*}$ maps; e histogram of the $\mathrm{R}_{2}{ }^{*}$ values. In the compartmental method, the sum of a Gaussian function (red) representing the cortex and a gamma function (purple) for the medullary values is fitted to the histogram. Arrow: distinct peak of medullary voxels, not visible on $3 \mathrm{~T}$ data. (Hoogduin et al. $\left.{ }^{(16)}\right)$.

\section{Arterial Spin Labelling}

Non-contrast enhanced renal perfusion imaging using ASL is an attractive approach for studying renal physiology and assessing renal diseases, and is well suited for the longitudinal monitoring of renal function. In ASL MRI, the arterial blood is used as an endogenous tracer ${ }^{(24)}$ by labelling it either with an adiabatic inversion RF pulse as in flow sensitive alternating inversion recovery (FAIR) or a pseudo-continuous RF pulse train as in pseudo continuous spin labelling (PCASL). Unfortunately, the intrinsically low SNR of ASL requires a large number of signal averages and correspondingly long imaging acquisition times, thus imposing critical limitations on its clinical application. After the inversion used in both FAIR and PCASL, the 
labelled spins that perfuse the area of interest disappear at a rate $R_{1}=1 / T_{1}$, thus the longer $\mathrm{T}_{1} \mathrm{~s}$ at $7 \mathrm{~T}$ increases the much needed SNR in ASL studies. It has been shown that $7 \mathrm{~T}$ can specifically benefit renal ASL perfusion imaging due to increased SNR, prolonged blood ${ }^{(25)}$ and renal tissue ${ }^{(8)}$ longitudinal relaxation times, and improved parallel imaging performance ${ }^{(26)}$. Theoretical simulations of renal perfusion SNR efficiency at $7 \mathrm{~T}$ and $3 \mathrm{~T}$ by using EPI as an imaging readout suggest that, compared to $3 \mathrm{~T}, 7 \mathrm{~T}$ can benefit renal ASL perfusion imaging by providing higher SNR efficiency even if longer repetition times are needed due to possible SAR constraints (Figure 11a) ${ }^{(27)}$. Initial 7 T studies suggest that there are no SAR issues for renal perfusion imaging using FAIR EPI with respiratory triggering acquisition ${ }^{(28,29)}$. The renal perfusion images from such a study are presented in Figure $11 b^{(27)}$.
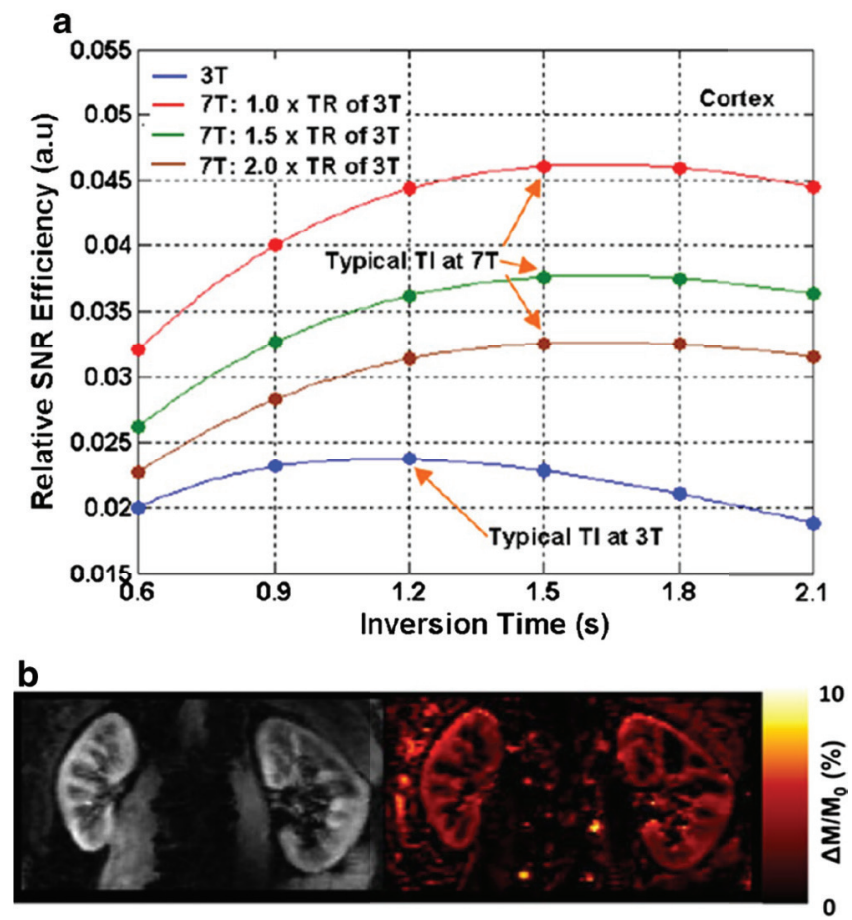

Figure 11. a Theoretical simulations of renal perfusion SNR efficiencies at $3 \mathrm{~T}$ and $7 \mathrm{~T}$ for renal perfusion imaging using FAIR EPI. TR represents repetition time. b One subject's proton (left) and normalized perfusion weighted (right) images from perfusion study using FAIR-EPI at $7 \mathrm{~T}$ with $2 \times 2 \times 5 \mathrm{~mm} 3$ resolution. $\Delta \mathrm{M}$ represents perfusion-weighted signal evaluated as the signal difference between label and control images, and Mo the fully relaxed renal tissue signal. (Li et al. ${ }^{(27)}$ ) . 
To avoid image distortions due to the sensitivity of an EPI readout to susceptibility effects and to take advantage of the dramatically increased perfusion SNR at $7 \mathrm{~T}$, the feasibility of single breath-hold renal ASL perfusion imaging was demonstrated by using a single shot-fast spin echo (ss-FSE) as the imaging readout (29) in combination with either pulsed ASL (PASL) using FAIR or PCASL. Although it is expected that PCASL can provide a higher perfusion SNR efficiency than FAIR, the limited coverage of the transceive body arrays used in these studies greatly limits the ability to label the blood in the descending aorta above the imaging field of view. This reduces the possible gains that PCASL theoretically can provide. In addition, compared to FAIR, PCASL is a much more power-intensive method, which poses additional problems at $7 \mathrm{~T}^{(24)}$. To limit SAR, the TR had to be lengthened with this approach, resulting in an increased imaging time that made it challenging to complete the sequence within a single breath hold ${ }^{(29)}$. Also the use of ss-FSE as readout yielded an increase in SAR compared to EPI, which necessitated the use of high parallel imaging factors and hyper-echoes for reducing the time averaged power delivered. In Figure 12, images acquired using FAIR and an ss-FSE readout are shown.
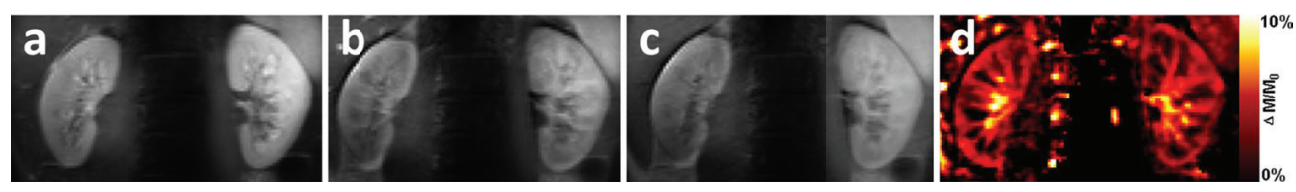

Figure 12. PASL images acquired with ss-FSE readout by Li et al. (29): a proton density; b control image; c labelling d perfusion weighted imaging normalized to proton density.

\section{Sodium Imaging}

Haneder et al. ${ }^{(30)}$ published the only work on renal sodium imaging at $7 \mathrm{~T}$. Due to a low sodium concentration, very short transverse relaxation times and intrinsically low MR sensitivity of the ${ }^{23} \mathrm{Na}$ nucleus in vivo sodium imaging is challenging. Theoretically, the ${ }^{23} \mathrm{Na}$ SNR at $7 \mathrm{~T}$ would be 2.33 times the SNR at $3 \mathrm{~T}^{\left({ }^{(30)}\right.}$. In addition, due to the lower Larmor frequency of the ${ }^{23} \mathrm{Na}$ nucleus resulting in a wavelength of about $45 \mathrm{~cm}$, sodium imaging at $7 \mathrm{~T}$ is hardly complicated by $\mathrm{B}_{1}$ inhomogeneity effects.

The transverse relaxation of the ${ }^{23} \mathrm{Na}$ nucleus can be described by a biexponential function, consisting of a fast and a slow component ${ }^{\left({ }^{0}, 31\right)}$. The aim of the study was to perform the first measure of the slow ${ }^{23} \mathrm{Na} \mathrm{T}_{2}{ }^{*}$ component in humans. In addition morphological ${ }^{23} \mathrm{Na}$ images were made (Figure 13). Imaging parameters for the morphological $3 \mathrm{D}$ spoiled GE sequence and the multi-echo $3 \mathrm{D}$ variable echo time (vTE) GE sequence for $\mathrm{T}_{2}{ }^{*}$ mapping are given in Table 6. The authors concluded that sodium imaging benefits from the increased field strength. The spatial resolution of morphological ${ }^{23} \mathrm{Na}$ images could be increased to $4 \times 4 \mathrm{~mm}^{2}$, 
compared to $5 \times 5 \mathrm{~mm}^{2}$ on $3 \mathrm{~T}^{\left({ }^{22}\right)}$, with improved image quality compared to lower field strengths. As in earlier studies, a cortico-medullary gradient could be observed with increasing sodium concentration in the medulla. The value was comparable: $4.1 \pm 0.35 \mathrm{mmol} / \mathrm{l} / \mathrm{mm}$ compared to $3.38 \pm 0.35 \mathrm{mmol} / \mathrm{l} / \mathrm{mm}$, as the same group measured earlier ${ }^{(32)}$. The measured slow component of $\mathrm{T}_{2}{ }^{*}$ values of 17.9 and 20.6 ms for cortex and medulla were in agreement with values published in animal studies on different field strengths (ranging from 11 to $36 \mathrm{~ms}$ on 2.1 to $17.6 \mathrm{~T}$ ) ${ }^{(30)}$.
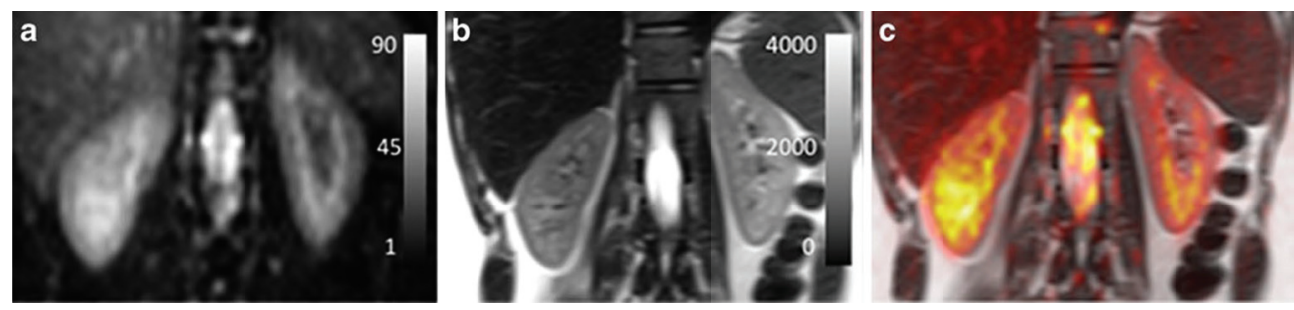

Figure 13. Coronal images acquired: $\mathbf{a}^{23} \mathrm{Na}$ image on $7 \mathrm{~T}$ with scale representing ${ }^{23} \mathrm{Na} \mathrm{SNR}$ in arbitrary units and $\mathbf{b}$ corresponding $\mathrm{T}_{2}$ weighted proton image in the same subject on $3 \mathrm{~T}$ with scale representing signal intensity in arbitrary units, $\mathbf{c} \mathrm{T}_{2}$ weighted image with overlaid colour encoded ${ }^{23} \mathrm{Na}$ image. (Haneder et al. ${ }^{(30)}$ ).

Table 6. Imaging parameters for the sodium imaging sequences ${ }^{\left({ }^{\circ}\right)}$.

\begin{tabular}{l|cc}
\hline Sequence & 3D spoiled GRE & Multi-echo 3D vTE GRE \\
\hline Slice orientation & coronal & coronal \\
TR [ms] & $49(38-61)$ & 75 \\
TE [ms] & 4.19 & $2.64 ; 4.93 ; 13.76 ; 19.18 ; 24.59 ; 30.01 ; 40.00 ;$ \\
& & $45.42 ; 55.00 ; 60.42$ \\
Nominal flip angle $\left[{ }^{\circ}{ }^{-}\right.$ & $\mathrm{NR}$ & $\mathrm{NR}$ \\
FOV [mm] & 256 & 256 \\
Voxel volume [mm] & $4 \times 4 \times 5$ & $4 \times 4 \times 15$ \\
Slices & 24 & 12 \\
Acquisition time [min] & $42(37-44)$ & 47 \\
\hline
\end{tabular}

NR: not reported.

\section{Challenges and Opportunities of Renal MRI at $7 \mathbf{T}$}

\section{RF Inhomogeneity}

One of the main difficulties in abdominal imaging at $7 \mathrm{~T}$ results from the short RF wavelength which negatively impacts transmit $B_{1}$ homogeneity and efficiency while increasing local SAR concerns. Transmit $\mathrm{B}_{1}$ homogeneity and efficiency are interrelated as increasing one often decreases the other when using standard static 
$\mathrm{RF}$ shimming techniques as discussed by Metzger et al. ${ }^{(14)}$. Inhomogeneous $\mathrm{B}_{1}$ can result in spatially dependent SNR and contrast. ${ }^{(4)}$ Loss in transmit efficiency can limit the achievable $\mathrm{B}_{1}{ }^{+}$needed for certain RF pulses such as those needed for refocusing and inversion. Finally, local SAR issues can greatly limit sequence and RF pulse design and timing in both cases. Even in low flip angle acquisitions, SAR limits become an issue as homogeneous solutions are often desired which, because of their decreased efficiency, require increased input power.

Although other solutions exist, most work on renal imaging at $7 \mathrm{~T}$ was performed using phase-only static RF-shimming ${ }^{(14,15,21)}$. RF-shimming consists of the use of a multiple channel transmit coil, usually 8 channels or more, where each can be driven with unique amplitudes, phases, and/or pulse waveforms. These channels are connected to a transmit/receive or transceive array, positioned close to the subject, similar to traditional receive arrays, but where each element transmits RF signals as well. This multi-channel RF system provides the flexibility needed to address the B1 transmit inhomogeneity and efficiency issues when imaging the kidneys at $7 \mathrm{~T}$.

Results presented in this paper have been acquired with three distinctly different coil arrays. Umutlu et al. used an $8 \times 1 \mathrm{~kW}$ amplifier setup to drive an 8 -channel array of microstrip elements (Figures 14 a and b) ${ }^{(15)}$ Hoogduin et al. used an $8 \times 2 \mathrm{~kW}$ amplifier setup to drive an array of eight fractionated dipole antennas (Figures $14 \mathrm{c}$ and $\mathrm{d}$ ) ${ }^{(33)}$ An extension of this array was recently presented where the dipole array is combined with a 16-element receive array, providing extra SNR. ${ }^{(34)}$ Metzger et al. and Li et al. made use of $16 \times 1 \mathrm{~kW}$ amplifiers to drive a 16-channel microstrip array (Figures $14 \mathrm{e}$ and f). ${ }^{(35)}$ Technical details of these arrays are provided in Figure 14. Sodium imaging results by Haneder et al. were acquired using a commercial 6-element spine array (Quality Electrodynamics, Mayfield Village, OH, USA). ${ }^{(30)}$

Using these multiple-channel coils, on each channel separately the phase of the RF signal can be adjusted to achieve $\mathrm{B}_{1}^{+}$homogeneity in a user-defined region of interest (ROI). In Figure 15 an example of phase-only shimming is shown. To calculate the desired phase for each RF-channel, the magnitude and phase estimates of each transmit channels' $\mathrm{B}_{1}^{+}$distribution are acquired. A relatively straightforward approach is to calculate the average $\mathrm{B}_{1}{ }^{+}$-phase over a user-defined ROI for each channel. Subsequently, this phase is subtracted from the transmit channel to obtain the same phase within the ROI for each channel ${ }^{(13)}$. Consequently, destructive interference of the fields within the ROI is minimized, maximizing the $\mathrm{B}_{1}^{+}$magnitude.

As demonstrated in the work by Metzger et al. ${ }^{(14)}$, multiple $\mathrm{B}_{1}^{+}$shim solutions can also be used, each optimized for specific requirement of RF pulses in the pulse sequence: homogeneity, efficiency or a trade-off between the two. These methods included a combination of both magnitude and phase shimming. This strategy was 

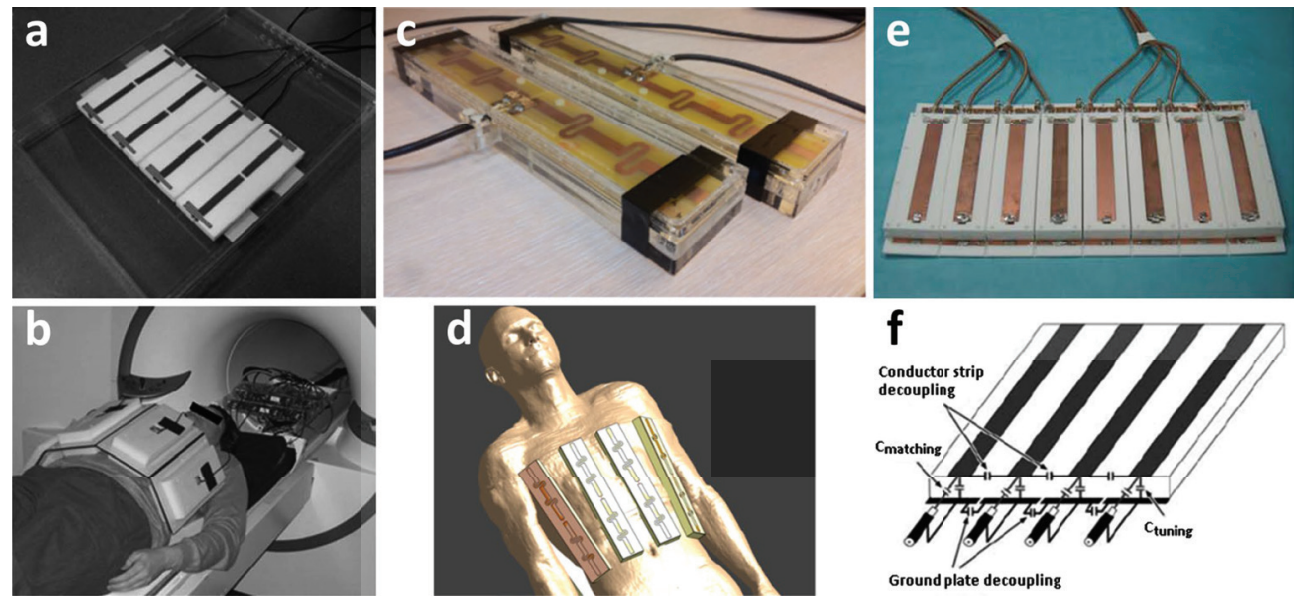

Figure 14. Different array coils and setups for renal $7 \mathrm{~T}$ imaging: a dorsal array of 8-channel array with microstrip meander elements used by Umutlu et al. ${ }^{(15)}$ These elements consist of a central conductor over a ground plane, which are connected to each other by capacitors at both ends of the element while the element is fed in the centre. At each end of the element, extra inductance is added by a meander in the central conductor that effectively lowers SAR and reduces inter-element coupling; $\mathbf{b}$ setup of a; $\mathbf{c}$ two coil elements and $\mathbf{d}$ setup used by Hoogduin et al. ${ }^{(33)}$ The array consists of eight 'fractionated dipole antennas'. Here, the legs of each dipole are divided into segments and the segments are interconnected by meanders (inductors). This element structure also reduces SAR levels and coupling in comparison to plain dipoles; e anterior array of 16-channel microstrip array used by Metzger et al. ${ }^{(26,35)}$. Here, a conductor is placed over a ground plate with capacitors connecting the two at both ends of the element (f). Capacitive coupling is used between the conductors and ground planes of adjacent elements to permit closer element spacing and higher element density. This element is driven from one side, over one of the connecting capacitors.
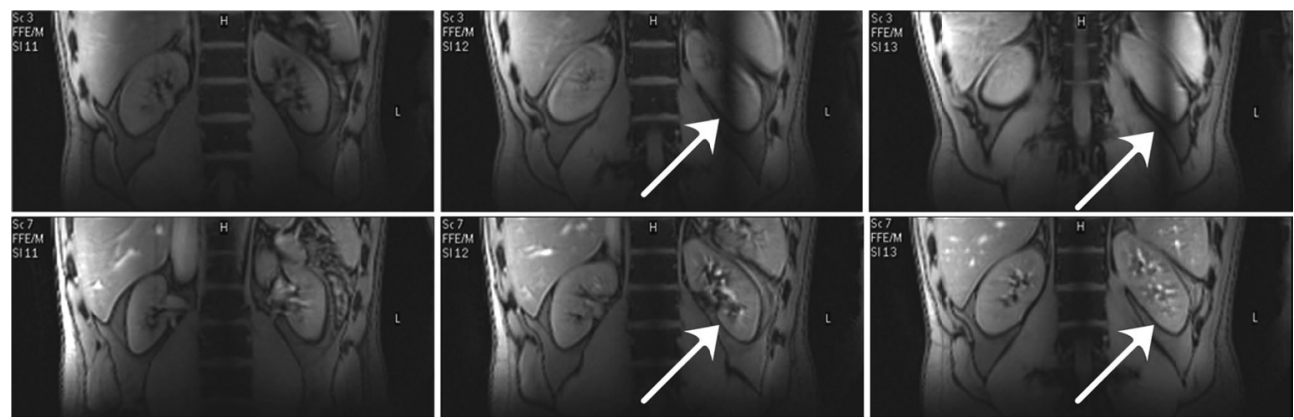

Figure 15. Survey pre (upper row) and post (lower row) RF shimming. Only phase shimming was performed. Arrows: region of destructive interference in the left kidney in two of three images acquired before RF shimming, disappearing after shimming. (Hoogduin et al. ${ }^{(16)}$ ).

used in non-CE MRA of the renal arteries at $7 \mathrm{~T}^{(14)}$. Here, an adiabatic inversion RF pulse was applied during the MRA sequence to suppress background signal. This pulse is relatively insensitive to $\mathrm{B}_{1}$ inhomogeneity but requires a $\mathrm{B}_{1}^{+}$magnitude 
above a certain threshold. In contrast, a conventional excitation pulse requires a homogeneous $\mathrm{B}_{1}^{+}$field to assure a constant flip angle throughout the ROI. This is important since areas with a small $\mathrm{B}_{1}^{+}$field can mimic occluded vessels in MR angiograms. To avoid these artefacts, RF shimming can be performed twice, once to optimize $\mathrm{B}_{1}^{+}$efficiency and once to optimize homogeneity or a trade-off between the two. Complex $\mathrm{B}_{1}^{+}$maps were acquired for each channel using a quick algorithm proposed by Van de Moortele ${ }^{(36)}$. Optimization was performed by phase or magnitude and phase. Although varying both parameters potentially yields the best results in terms of $\mathrm{B}_{1}^{+}$homogeneity and magnitude, sometimes most of the RF power is distributed over a few channels. This yields the risk of exceeding the local specific absorption (SAR) limits. Therefore phase-only optimization was preferentially used. However, when only small flip-angles are desired, the risk of exceeding SAR limits is smaller and magnitude can be included in the calculations.

Alternatives for RF shimming are for example, transmit SENSE (37) and time interleaved acquisition of modes (TIAMO) ${ }^{(38)}$. In transmit SENSE, using the transmit profile of each coil and a user defined gradient trajectory, a unique RF-waveform is calculated for each transmit channel. Consequently, transmit SENSE requires hardware that is capable of producing an arbitrary RF waveform for individual channels. In TIAMO, two acquisitions are performed with different excitation modes of the RF pulse for example, a $45^{\circ}$ phase increment along RF coils followed by a $90^{\circ}$ increment. Using parallel imaging techniques, the two acquisitions can be acquired in the same time as needed for a single image ${ }^{\left({ }^{38}\right)}$. Using GRAPPA the final image is reconstructed from the two acquisitions.

\section{SAR Limitations}

MR imaging at $7 \mathrm{~T}$ is associated with higher SAR levels. More power is needed since the penetration depth becomes smaller. This is because the electric fields that are induced are larger, which also results in higher SAR levels. However, at $7 \mathrm{~T}$, local transmit coil arrays are being used. These arrays are positioned much closer to the patient and are therefore much more efficient. The local transmit array of dipole antennas only needs $8 \times 2 \mathrm{~kW}$ to reach the same B1 levels for which a $3 \mathrm{~T}$ body coil requires 2x16 kW. So although $7 \mathrm{~T}$ Bo fields are associated with higher SAR levels, this does not apply for global SAR since much more efficient local transmit arrays are being used at $7 \mathrm{~T}$. Nevertheless, to ensure that global SAR does not exceed limits, multiple groups implemented real-time power monitoring ${ }^{(14,15)}$.

However, the use of local transmit arrays can lead to large variations in energy deposition ${ }^{(14)}$. Therefore, also local SAR has to be taken into account. The IEC guidelines ${ }^{(39)}$ prescribe a limit for the maximum allowed $10 \mathrm{~g}$ averaged local SAR value anywhere in the exposed region (peak local SAR). The exact limit depends on the body part and the surveillance mode but ranges from 10 to $40 \mathrm{~W} / \mathrm{kg}$. Unlike 
global SAR, local SAR cannot be measured; it has to be calculated from numerically simulated field distributions and the applied phase and amplitude settings of the transmit array. An example of such simulated local SAR distributions is provided in Figure 16 for two different shim settings, both optimized for kidney imaging. The peak local SAR in these simulated distributions dictates the minimum repetition time, taking into account an uncertainty margin because of inter-subject variability. ${ }^{(40)}$ Thus, the peak local SAR limits are what constrain $7 \mathrm{~T}$ kidney imaging.
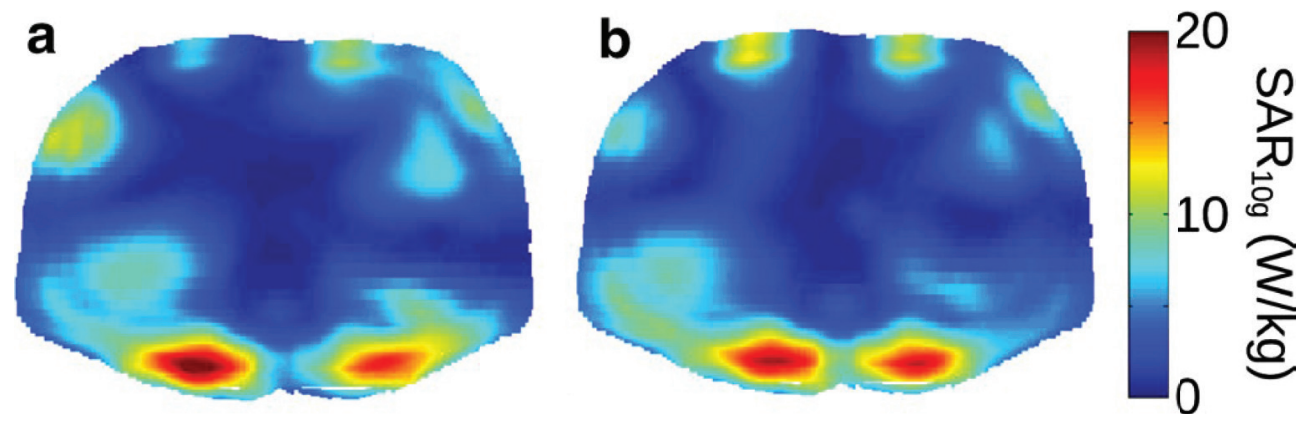

Figure 16. Local SAR distributions (10 g averaged) for two phase-amplitude settings that are both designed for constructive interference of $\mathrm{B} 1$ in the kidneys. Distributions are in the transverse plane crossing the maximum value in the distribution. Values are for $8 \times 800 \mathrm{~W}$, with $1 \%$ duty cycle. (Raaijmakers, unpublished results)

An important method with which to reduce SAR is parallel imaging. Since fewer phase encoding steps are required, less RF-pulses are needed and acquisition time is decreased. However, it does reduce SNR - so in principle it enables us to acquire the same SNR at the same SAR in less time on high fields as compared to low fields ${ }^{(41)}$. Other options to reduce SAR include reducing echo train length or increasing repetition time, but this directly influences acquisition time ${ }^{(12)}$. Simply decreasing the flip angle has a significant influence on SAR, since SAR changes quadratically with flip angle for a given RF pulse ${ }^{(12)}$. However, a decreased flip angle also influences image contrast. Alternatively, the power required by the RF pulse can be minimized, for example using variable-rate selective excitation (VERSE) ${ }^{(42)}$ or, when adiabatic pulses are desired, using gradient-modulated offset-independent adiabaticity (GOIA) ${ }^{(43)}$.

TSE sequences have high energy deposition due to the refocusing $180^{\circ}$ pulse trains used. It is possible to reduce the flip angles in this pulse train using transition between pseudo steady states (TRAPS) ${ }^{(44)}$ or using hyperechoes ${ }^{(45)}$. Both techniques employ the fact that image contrast is stored in the centre of k-space. While the centre of k-space should be sampled with a sufficiently large flip angle, smaller flip angles can be used for the periphery. 


\section{Conclusion and Discussion}

Although the feasibility of numerous sequences on $7 \mathrm{~T}$ in the kidney has been demonstrated, there are still a number of difficulties that need to be overcome. SAR limitations seem to impose most restrictions on sequence optimization. Techniques as parallel imaging and the use of hyperechoes have partially overcome these problems, but come at the cost of SNR. RF inhomogeneity can be successfully dealt with using RF shimming, and more advanced techniques are being developed, potentially leading to superior $\mathrm{B}_{1}^{+}$homogeneity ${ }^{(37,38)}$.

Anatomical imaging seems to suffer most from SAR constraints and to a lesser degree from RF inhomogeneity. In $\mathrm{T}_{1}$ weighted sequences, resolutions of about $1 \mathrm{~mm}$ are reached within about half a minute acquisition time. In conventional clinical imaging at $3 \mathrm{~T}$, results are comparable in terms of spatial resolution and scan duration ${ }^{(46)}$. $T_{2}$ weighted imaging, however, is strongly impaired by SAR constraints, resulting in artefacts or increased imaging times, although spatial resolution could be slightly improved.

Excellent image quality of the renal vascular system at $7 \mathrm{~T}$ using non-CE sequences suggest a bright future for this technique. Although the feasibility was shown by two groups ${ }^{(14,19)}$, no direct comparison between non-CE and CE MRA is available. Comparison of the CNRs measured for TOF MRA and CE MRA yields a two fold increase in CNR for CE MRA (28 versus a range of 45 to 60), but quality scores barely differed (4.7 versus 4.8$)^{(19,20)}$. In addition, no information on dosage and timing of contrast agent administration is yet available and no pathology was studied.

Benefits of functional renal MRI at $7 \mathrm{~T}$ potentially are large and address the important clinical need for techniques that can better assess local and split renal function. Theoretically, both techniques currently applied in the kidneys profit from the high field strength. BOLD MRI benefits from increased sensitivity to susceptibility effects, while ASL utilizes the increase in $\mathrm{T}_{1}$ relaxation times. Promising results are obtained in sodium imaging. Since the RF wavelength related to the sodium nucleus is about $45 \mathrm{~cm}$ in tissue, transmit inhomogeneity is not an issue. Thanks to the increased SNR at $7 \mathrm{~T}$ compared to $3 \mathrm{~T}$, resolution could be increased by $20 \%{ }^{\left({ }^{30}\right)}$.

In conclusion, although numerous challenges still have to be overcome, the future of renal 7T MRI is promising, especially for functional imaging techniques. 


\section{Bibliography}

1. Robitaille PML, Abduljalil AM, Kangarlu A, Zhang X, Yu Y, Burgess R, et al. Human magnetic resonance imaging at 8 T. NMR Biomed. 1998;11(6):263-5.

2. Ashman CJ, Farooki S, Abduljalil AM, Chakeres DW. In vivo high resolution coronal MRI of the wrist at 8.o tesla. J Comput Assist Tomogr. 2002;26(3):387-91.

3. Metzger GJ, Van de Moortele PF, C.J. S, J.T. V, K. U, editors. Local B1 Shimming for Imaging the Prostate at 7 Tesla. Proceedings of the 15th scientific meeting, International Society for Magnetic Resonance in Medicine; 2007. Berlin.

4. Vaughan JT, Snyder CJ, Delabarre LJ, Bolan PJ, Tian J, Bolinger L, et al. Whole-body imaging at 7T: Preliminary results. Magn Reson Med. 2009;61(1):244-8.

5. Snyder CJ, DelaBarre L, Metzger GJ, van de Moortele PF, Akgun C, Ugurbil K, et al. Initial results of cardiac imaging at 7 Tesla. Magn Reson Med. 2009;61(3):517-24.

6. Dieringer MA, Renz W, Lindel T, Seifert F, Frauenrath T, von Knobelsdorff-Brenkenhoff F, et al. Design and application of a four-channel transmit/receive surface coil for functional cardiac imaging at 7T. J Magn Reson Imaging. 2011;33(3):736-41.

7. Moser E, Stahlberg F, Ladd ME, Trattnig S. 7-T MR-from research to clinical applications? NMR Biomed. 2012;25(5):695-716.

8. Li X, Bolan PJ, Ugurbil K, Metzger GJ. Measuring renal tissue relaxation times at 7 T. NMR Biomed. 2015;28(1):63-9.

9. Shen Y, Goerner FL, Snyder C, Morelli JN, Hao D, Hu D, et al. T1 relaxivities of gadolinium-based magnetic resonance contrast agents in human whole blood at 1.5, 3, and 7 T. Invest Radiol. 2015;50(5):330-8.

10. Kalavagunta C, Michaeli S, Metzger GJ. In vitro Gd-DTPA relaxometry studies in oxygenated venous human blood and aqueous solution at 3 and 7 T. Contrast Media Mol Imaging. 2014;9(2):169-76.

11. Noebauer-Huhmann IM, Szomolanyi P, Juras V, Kraff O, Ladd ME, Trattnig S. Gadolinium-based magnetic resonance contrast agents at 7 Tesla: in vitro T1 relaxivities in human blood plasma. Invest Radiol. 2010;45(9):554-8.

12. McRobbie DW, Moore EA, Graves MJ, R. PM. MRI From Picture to Proton. Second ed. Cambridge: Cambridge University Press; 2015.

13. Metzger GJ, Snyder C, Akgun C, Vaughan T, Ugurbil K, Van De Moortele PF. Local $\mathrm{B} 1+$ shimming for prostate imaging with transceiver arrays at $7 \mathrm{~T}$ based on subjectdependent transmit phase measurements. Magn Reson Med. 2008;59(2):396-409.

14. Metzger GJ, Auerbach EJ, Akgun C, Simonson J, Bi X, Uğurbil K, et al. Dynamically applied B1 + shimming solutions for non-contrast enhanced renal angiography at 7.0 tesla. Magn Reson Med. 2013;69(1):114-26.

15. Umutlu L, Orzada S, Kinner S, Maderwald S, Brote I, Bitz AK, et al. Renal imaging at 7 Tesla: Preliminary results. Eur Radiol. 2011;21(4):841-9. 
16. Hoogduin H, Raaijmakers A, Visser F, Luijten P, editors. Initial experience with BOLD imaging of the kidneys at $7 \mathrm{~T}$. Proceedings of the 22nd scientific meeting, International Society for Magnetic Resonance in Medicine; 2014. Milan.

17. Grinstead JW, Rooney W, Laub G, editors. The Origins of Bright Blood MPRAGE at 7 Tesla and a Simultaneous Method for T1 Imaging and Non-Contrast MRA. Proceedings of the 18th scientific meeting, International Society for Magnetic Resonance in Medicine; 2010. Stockholm.

18. Umutlu L, Kraff O, Orzada S, Fischer A, Kinner S, Maderwald S, et al. Dynamic contrastenhanced renal MRI at 7 Tesla preliminary results. Invest Radiol. 2011;46(7):425-33.

19. Umutlu L, Maderwald S, Kraff O, Kinner S, Schaefer LC, Wrede K, et al. New look at renal vasculature: 7 tesla nonenhanced T1-weighted FLASH imaging. J Magn Reson Imaging. 2012;36(3):714-21.

20. Umutlu L, Maderwald S, Kinner S, Kraff O, Bitz AK, Orzada S, et al. First-pass contrastenhanced renal MRA at 7 Tesla: Initial results. Eur Radiol. 2013;23(4):1059-66.

21. Brinkmann I, Darji N, Speck O, Bock M, editors. BOLD MRI of the Kidneys under water loading at 7 Tesla using parallel Transmission and RF shimming of individual slices. Proceedings of the 22nd scientific meeting, International Society for Magnetic Resonance in Medicine; 2014. Milan.

22. Ebrahimi B, Gloviczki M, Woollard JR, Crane JA, Textor SC, Lerman LO. Compartmental analysis of renal BOLD MRI data: introduction and validation. Invest Radiol. 2012;47(3):175-82.

23. Vink EE, de Boer A, Hoogduin HJ, Voskuil M, Leiner T, Bots ML, et al. Renal BOLDMRI relates to kidney function and activity of the renin-angiotensin-aldosterone system in hypertensive patients. J Hypertens. 2015;33(3):597-603; discussion -4.

24. Wong EC. An introduction to ASL labeling techniques. J Magn Reson Imaging. 2014;40(1):1-10.

25. Zhang X, Petersen ET, Ghariq E, De Vis JB, Webb AG, Teeuwisse WM, et al. In vivo blood T(1) measurements at 1.5 T, 3 T, and 7 T. Magn Reson Med. 2013;70(4):1082-6.

26. Snyder CJ, Delabarre L, Moeller S, Tian J, Akgun C, Van de Moortele PF, et al. Comparison between eight- and sixteen-channel TEM transceive arrays for body imaging at 7 T. Magn Reson Med. 2012;67(4):954-64.

27. Li X, Ugurbil K, Metzger G, editors. Theoretical evaluation of ultrahigh field benefits to non-contrast enhanced renal perfusion imaging using FAIR-EPI. Proceedings of the 21th scientific meeting, International Society for Magnetic Resonance in Medicine; 2013. Salt Lake City.

28. Li X, Snyder C, Van de Moortele PF, Ugurbil K, Metzger G, editors. Non-contrast enhanced human renal perfusion imaging using arterial spin llabeling at $7 \mathrm{~T}$ : initial experience. Proceedings of the 2oth scientific meeting, International Society for Magnetic Resonance in Medicine; 2012. Melbourne. 
29. Li X, Snyder C, Van de Moortele PF, Ugurbil K, Metzger G, editors. Feasibility of single breath-hold renal perfusion imaging at $7 \mathrm{~T}$. Proceedings of the 21st scientific meeting, International Society for Magnetic Resonance in Medicine; 2013. Salt Lake City.

30. Haneder S, Juras V, Michaely HJ, Deligianni X, Bieri O, Schoenberg SO, et al. In vivo sodium $(23 \mathrm{Na})$ imaging of the human kidneys at 7 T: Preliminary results. Eur Radiol. 2014;24(2):494-501.

31. Zollner FG, Konstandin S, Lommen J, Budjan J, Schoenberg SO, Schad LR, et al. Quantitative sodium MRI of kidney. NMR Biomed. 2016;29(2):197-205.

32. Haneder S, Konstandin S, Morelli JN, Nagel AM, Zoellner FG, Schad LR, et al. Quantitative and qualitative $23 \mathrm{Na}$ MR imaging of the human kidneys at $3 \mathrm{~T}$ : Before and after a water load. Radiology. 2011;260(3):857-65.

33. Raaijmakers AJ, Italiaander M, Voogt IJ, Luijten PR, Hoogduin JM, Klomp DW, et al. The fractionated dipole antenna: A new antenna for body imaging at 7 Tesla. Magn Reson Med. 2015.

34. Voogt IJ, Klomp D, Hoogduin H, Luttje MP, Luijten P, van den Berg CAT, et al., editors. Combined 8-Channel Transceiver Fractionated Dipole Antenna Array with a 16-Channel Loop Coil Receive Array for Body Imaging at 7 Tesla. Proceedings of the 23rd scientific meeting, International Society for Magnetic Resonance in Medicine; 2015. Toronto.

35. Metzger GJ, van de Moortele PF, Akgun C, Snyder CJ, Moeller S, Strupp J, et al. Performance of external and internal coil configurations for prostate investigations at 7 T. Magn Reson Med. 2010;64(6):1625-39.

36. Van de Moortele PF, K. U, editors. Very Fast Multi Channel B1 Calibration at High Field in the Small Flip Angle Regime. Proceedings of the 17th scientific meeting, International Society for Magnetic Resonance in Medicine; 2009. Honolulu.

37. Katscher U, Börnert P, Leussler C, Van den Brink JS. Transmit SENSE. Magn Reson Med. 2003;49(1):144-50.

38. Orzada S, Maderwald S, Poser BA, Bitz AK, Quick HH, Ladd ME. RF excitation using Time Interleaved Acquisition of Modes (TIAMO) to address B1 inhomogeneity in highfield MRI. Magn Reson Med. 2010;64(2):327-33.

39. IEC. 60601-2-33 Medical Electrical Equipment. Part 2-33 Particular requirements for the safety of Magnetic Resonance equipment for medical diagnosis 2nd ed. Geneva: International Electrotechnical Commision; 2002.

40. Ipek O, Raaijmakers AJ, Lagendijk JJ, Luijten PR, van den Berg CA. Intersubject local SAR variation for $7 \mathrm{~T}$ prostate MR imaging with an eight-channel single-side adapted dipole antenna array. Magn Reson Med. 2014;71(4):1559-67.

41. Schmitz BL, Aschoff AJ, Hoffmann MH, Gron G. Advantages and pitfalls in $3 \mathrm{~T}$ MR brain imaging: a pictorial review. AJNR Am J Neuroradiol. 2005;26(9):2229-37.

42. Conolly S, Nishimura D, Macovski A, Glover G. Variable-rate selective excitation. J Magn Reson (1969). 1988;78(3):440-58. 
43. Tannus A, Garwood M. Adiabatic pulses. NMR Biomed. 1997;10(8):423-34.

44. Hennig J, Weigel M, Scheffler K. Multiecho sequences with variable refocusing flip angles: optimization of signal behavior using smooth transitions between pseudo steady states (TRAPS). Magn Reson Med. 2003;49(3):527-35.

45. Hennig J, Scheffler K. Hyperechoes. Magn Reson Med. 2001;46(1):6-12.

46. De Keyzer F, Thoeny HC. Renal and perfusion imaging at 3 T. Top Magn Reson Imaging. 2010;21(3):157-63. 



\section{Chapter 9}

Discussion and Conclusion

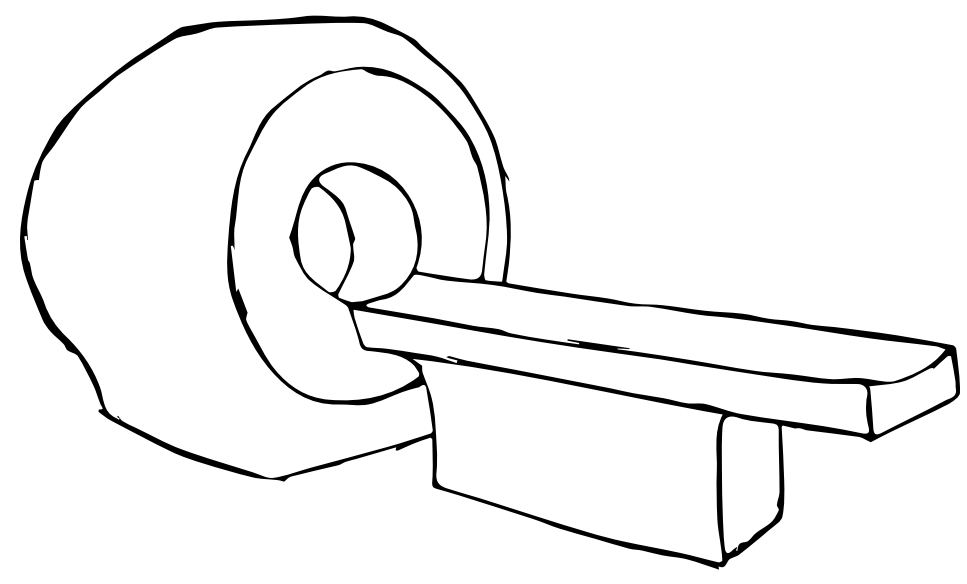




\section{Discussion}

\section{The Future of Renal MRI}

The first report on MRI of a human kidney dates back to 1981.(1) Although this report is only cited 12 times, the title remains relevant: "Renal cyst or tumour? Differentiation by whole-body nuclear magnetic resonance imaging." Cyst or tumor? Still, that is one of the main questions asked to radiologists if a renal MRI is requested. Despite the remaining challenges, I believe that in the near future clinical indications for renal MRI will extend beyond the detection of anatomical abnormalities. As a first step, MRI might prove its use as "virtual biopsy" for kidney patients. For patients, a renal biopsy is a painful procedure which requires them to remain in hospital in supine position for 6 hours. Furthermore, complications like hemorrhage can occur (0.3-10\% of cases).(2)

In general, clinically relevant findings on biopsies in both kidney transplantation patients as well as patients with chronic kidney disease (CKD), typically are of inflammatory or fibrotic origin. Inflammation can be treated, while detection of fibrosis is important for prognosis. MRI has the potential to detect both. Multiple studies have found correlations between fibrosis and increased renal $\mathrm{T}_{1}$ or restriction of renal diffusion.(3-6) Inflammation has also been shown to induce changes measureable using MRI: changes in perfusion, $(7)$ restricted diffusion $(8,9)$ and increased $\mathrm{T}_{1}$ and $\mathrm{T}_{2}(10)$. However, specificity of MRI remains a point of concern. Fibrosis and inflammation induce the same changes in diffusion constants and $\mathrm{T}_{1}$. In this context, $\mathrm{T}_{2}$ is an interesting measure, since it would be expected to decrease in response to fibrosis, while it would increase in inflammatory conditions.(10-13) In general, literature on changes in $\mathrm{T}_{2}$ in response to parenchymal renal diseases is limited. In chapter 7 , we report a decrease in $\mathrm{T}_{2}$ in a fibrotic kidney. However, this single case report cannot be conclusive, since only a single kidney was analyzed and other factors influencing $\mathrm{T}_{2}$, like inflammatory changes and hemorrhages, were present. More research is needed to confirm whether the addition of $\mathrm{T}_{2}$ mapping can increase the specificity of multiparametric MRI.

Early after kidney transplantation, discrimination between acute tubular necrosis (ATN) and acute rejection (AR) of the allograft is crucial to guide proper treatment. Especially BOLD MRI seems promising in discrimination between those conditions, since AR is associated with decreased medullary $\mathrm{R}_{2}^{*}$ values while cortical $\mathrm{R}_{2}{ }^{*}$ values tend to be increased in ATN.(14) This difference in $\mathrm{R}_{2}{ }^{*}$ between ATN and $\mathrm{AR}$ probably arises from the marked reduction in perfusion demonstrated in AR compared to ATN, especially in the medulla.(15) A severe reduction in perfusion leads to a decrease in blood volume and therefore hemoglobin (both oxygenated and deoxygenated), resulting in a decreased $\mathrm{R}_{2}^{*}$.

Considering the abovementioned capabilities and limitations of renal MRI, 
several possible clinical implementations of renal MRI in clinical care are possible. Renal multiparametric MRI might be used to indicate, replace or guide renal biopsies. A biopsy might be indicated if an MRI shows inflammatory changes or if it is inconclusive. Conversely, if the MRI shows a fibrotic kidney, a biopsy is not expected to yield any further clinical relevant information. Currently, the ongoing ACRADYS (Assessment of Chronic Renal Allograft DYSfunction with MRI) study in our center focuses on kidney transplant patients whose transplant is biopsied later than 3 months post transplantation. Participants undergo an MRI to enable a comparison of the results of the biopsy and the MRI. While this study is focused on chronic changes in kidney transplants, a similar study design may be extended to other domains, for example acute conditions following transplantation and acute or chronic disease in native kidneys. Several studies in different renal diseases have been reported $(3-5,16-18)$ mostly assessing a limited number of MRI sequences simultaneously. If other MRI sequences are introduced as well, multiparametric evaluation could allow for recognition of patterns in MR parameters characteristic for different pathologies.

Another promising application of MRI is risk stratification in patients who often have subclinical renal disease such as patients with diabetes mellitus or hypertension. In chapter 6, we showed that medullary $\mathrm{R}_{2}^{*}$ in patients with hypertension increased with decreasing estimated glomerular filtration rate (eGFR), indicating medullary hypoxia. Markers of activity of the renin angiotensin aldosterone system (RAAS) also correlated with renal cortical and medullary $\mathrm{R}_{2}{ }^{*}$. Another study described a correlation between cortical $R_{2}^{*}$ value and corticomedullary differentiation in terms of $\mathrm{R}_{2}{ }^{*}$ and future eGFR decline.(19) In kidney transplantation patients, reduced perfusion as measured with ASL has been shown to predict worse renal function one year post-transplantation in patients with delayed graft function.(16) Those promising findings indicate that functional MRI can be used to determine risk of progression in kidney disease patients. To confirm this, and to determine the added value of other MRI measures, large, long-term studies are required.

Risk stratification is clinically important to enable personalization of treatment. This will gain relevance if targeted drugs are developed in the future. Because of the expected high costs of targeted medication, eligible patients would ideally be selected in advance. Alternatively, drug effectiveness could be assessed shortly after initiation of treatment. Renal MRI might play a role in that. Last, renal MRI might be used to select patients at high risk of progression for inclusion in drug trials, reducing the required sample size.

In research settings MRI can be used to obtain functional information which cannot otherwise be obtained noninvasively. In chapter 5, BOLD MRI was used to evaluate the effect of renal denervation (a potential treatment for hypertension) on renal oxygenation. Despite a clinically significant drop in blood pressure in 
a subgroup of patients, no change in renal $\mathrm{R}_{2}{ }^{*}$ was detected in those responders. This was in line with results of another study (20) which reported no difference in $\mathrm{R}_{2}{ }^{*}$ between hypertensives and controls. Only after administration of furosemide, the drop in medullary $\mathrm{R}_{2}{ }^{*}$ found in healthy controls was reduced in hypertensives, indicating alterations in medullary function.(20)

The use of 7 T MRI might prove to be of additional value in research settings. As discussed in chapter $\boldsymbol{8}$, some functional MRI techniques including BOLD and ASL MRI are expected to benefit from high field MR systems. The BOLD effect increases at higher field strength, increasing sensitivity to subtle changes in oxygenation. For ASL, a recent study showed that good-quality perfusion images could be obtained with only 4 signal averages, compared to around 20 at $3 \mathrm{~T}$, which results in a significant reduction of scan time.(21, 22) Furthermore, 7 T ASL might allow for quantification of medullary perfusion, which is considered unreliable at 1.5 and $3 \mathrm{~T}$ currently (22) since the ASL label has mostly decayed by the time the labeled bolus enters the renal medulla. At $7 \mathrm{~T}$, the longitudinal relaxation time of blood is significantly longer (23), resulting in an increase of signal in the medulla. Nevertheless, $7 \mathrm{~T}$ renal MRI has not yet been widely adopted, probably due to the challenges associated with (abdominal) imaging at this field strength.

\section{Challenges in Clinical Adoption of Renal MRI}

BOLD, DWI and ASL MRI all existed before 2000. Now, 20 years later, clinical use of those techniques in clinical care is limited to some well-defined areas such as malignancies and brain infarctions. Clinical implementation of new inventions is notoriously slow - also in MRI. Why?

In case of MRI, it might have to do with its complexity. Renal volume arguably the most straightforward measure one can obtain using an MRI scanner - has been the first official biomarker of renal health recognized by the FDA.(24) Even measurement of $\mathrm{T}_{1}$ or $\mathrm{T}_{2}$, which $\mathrm{I}$ in the introduction dared to describe as "an actual, physical property of tissue", does not yield the same values when measured with two different protocols or in two different centers. In a recent recommendation paper on $\mathrm{T}_{1}$ and $\mathrm{T}_{2}$ mapping(25), four different techniques for measurement of $\mathrm{T}_{1}$ were discussed - and those were only the most commonly used approaches. Within such a technique, there are dozens of different parameters which can be tuned, all depending on each other and with a more or less substantial influence on the $\mathrm{T}_{1}$ measured. And don't forget patient related factors. For example: does hydration state matter or smoking before the examination? Should the patient be fasting prior to the examination? Is he capable of holding his breath? Does it matter that she had a heavy training yesterday? For most techniques we simply don't know yet. For example: BOLD MRI is thought to be sensitive to hydration status, but this has only be shown for an acute water load of $20 \mathrm{~mL} / \mathrm{kg}$ in 15 minutes (or $1.4 \mathrm{~L}$ for a $70 \mathrm{~kg}$ 
subject) (26), which can hardly be considered physiological.

Considering the results of our repeatability study (chapter 3) the influence of those parameters is probably limited. We only loosely controlled hydration state and salt intake, and yet reproducibility of most measurements was at least satisfactory, and in line with other reports. Nevertheless, the repeatability study was not designed to measure the influence of physiological variations and dedicated studies should be performed to assess this. What if those studies show that influence physiological variations is substantial? How would kidney MRI then ever be used in clinical reality, where patients are quite likely to forget or misunderstand your instructions - or simply consider them too cumbersome? If influence of real-life physiological variations on a particular MR measurement is larger than, or as large as the influence of the pathologies we are interested in, we might have to conclude that this MR measurement is not suitable for use in clinical care.

In the UK, an interesting study is currently being performed to tackle the aforementioned technical challenges. In more than ten centers with scanners of three different vendors, harmonized multiparametric kidney protocols are acquired in the same group of volunteers (the so called "travelling kidneys"). (https://www. nottingham.ac.uk/research/groups/spmic/research/uk-renal-imaging-network/ ukrin-maps.aspx) Furthermore, phantoms with known and fixed properties will be scanned. "Harmonized or standardized multiparametric protocols" might sound easy and straightforward, but it is not. Different vendors describe their acquisition sequences differently. For example, one specific vendor typically only reports a reconstructed voxel size, which might differ from the voxel size which actually is acquired. Calculation of the acquired voxel size is not straightforward since it depends on numerous parameters but is crucial to perform the exact same scan on an MR system of another vendor. Furthermore, due to differences in both hardware and software, scans often cannot even be performed with the exact same settings on different MR systems. Consequently, across-vendor harmonization of scan protocols is a time-consuming task. The relevant question is to what extent acquisition protocols should be harmonized to obtain comparable results. A recent study on cross-vendor stability of some brain MR measurements (including hippocampal volume, resting state fMRI and DTI) yielded promising results with CoVs below $7.8 \%$ and intrascanner variability comparable to interscanner variability, even though perfect harmonization of scan protocols was not possible.(27) Instead of pursuing perfect harmonization of scan protocols, MR systems might be calibrated based on functional measurements on phantoms with known properties. 


\section{Outlook: Technical Developments}

\section{MRI Acquisition - Decreasing Acquisition Time}

A typical renal multiparametric MRI protocol currently takes around one hour. For clinical use in patients, this is too long and uncomfortable. Therefore, efforts should be taken to reduce acquisition time.

Fast scanning would also be desirable for different reasons. The kidneys move with respiration and motion during acquisition, resulting in respiratory artefacts. This problem is usually solved by either breath holding, respiratory triggering or the use of very quick readouts (for example echo planar imaging (EPI) readouts of a few hundred milliseconds). Breath holds however are limited in length, since patients cannot be expected to hold their breath for longer than $\sim 10$ seconds. Respiratory triggering heavily increases scan time, since the acquisition is effectively partitioned in small pieces, and in each respiratory cycle ( $\sim 6$ seconds) only one of those partitions can be measured. Furthermore, those EPI readouts can only acquire one slice at a time, obtaining whole kidney coverage takes time. When very quick readouts are used, the images have to be realigned after image acquisition. Although numerous strategies exist to achieve this, it might be challenging when there is a significant change or even inversion of contrast during the scan (for example in $\mathrm{T}_{1}$ mapping or DCE MRI). In chapter 2, we proposed to use Dixon fat images, which exhibit a constant contrast throughout the DCE examination, for realignment. Indeed, this approach outperformed realignment on Dixon water images, which did show contrast changes.

Acceleration of MRI acquisitions is a large area of research. One promising technique is compressed sensing.(28) This technique exploits "sparsity" in MR images. It is most easily explained in the time domain. Imagine a DCE acquisition, where contrast is injected while continuously acquiring images (see chapter 2 and 3). Although image contrast changes due to inflow of contrast agent, most parts of the image are pretty similar over time. Therefore, it is not necessary to acquire the entire image at every timepoint when using data from other timepoints to fill in the missing information. The technique has some limitations: large changes in contrast might not be captured correctly (see chapter 3) so it has to be used with care. On recent software releases of all major vendors, compressed sensing is commercially available.

Another approach to reduce scan time is the use of high-density receive arrays. $(29,30)$ On the scanner where most of the research presented in this thesis is performed, we use a receive antenna with 28 channels. This allows a decrease in scan time by a factor (called the acceleration factor) of 2-3, depending on the image orientation and field of view. Theoretically, the number of receive channels can be increased indefinitely, with up to 256-channel arrays currently being developed.(30) With those high-density receive arrays, acceleration factors of at least 10 should be 
achievable. An acceleration factor of 10 means that a BOLD image, which with the current acceleration factor of 2 takes $\sim 50$ seconds to acquire, can be acquired in a single breath hold of $\sim 10$ seconds. The EPI readouts now used for $\mathrm{T}_{1}$ and $\mathrm{T}_{2}$ mapping could become $3 \mathrm{D}$ instead of $2 \mathrm{D}$, acquiring the entire kidney volume in the same scan time previously needed for a single slice (Figure 1). Using those techniques, acquisition of a multiparametric dataset including planning and patient preparation in a conventional scan slot of 35 minutes is within reach.

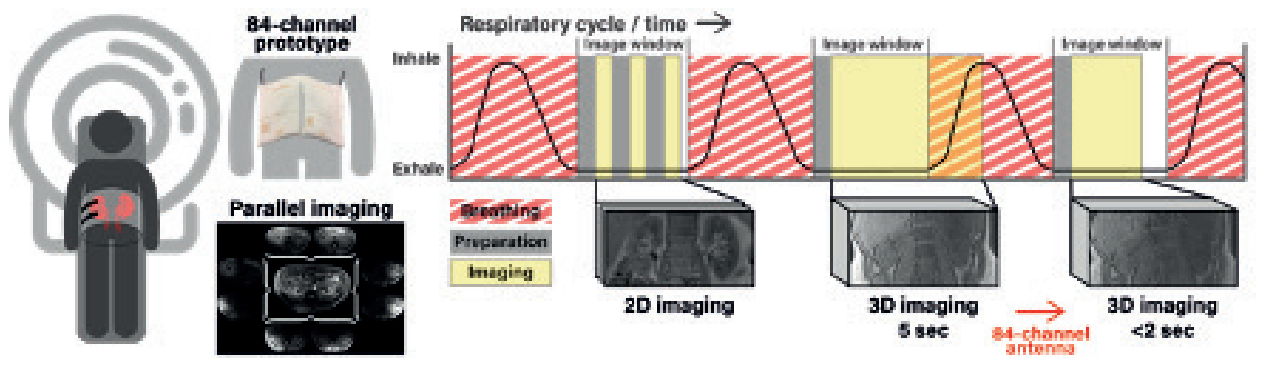

Figure 1. By parallel imaging, 256 images can be acquired simultaneously, each with their own field of view, reducing acquisition time. These images can be combined in a single image using a technique called SENSE (sensitivity encoding) or GRAPPA (generalized autocalibrating partial parallel acquisition). Currently, multiple 2D images are acquired to obtain full kidney coverage, for which multiple breathing cycles are needed (first part graph). 3D imaging, or the acquisition of an entire volume instead of one slice, is not possible because it does not fit in one image window, which leaves the image corrupted by motion (middle part graph). A prototype antenna with 84 channels, already enabled acquisition of a $3 \mathrm{D}$ image in less than 2 seconds (SENSE acceleration factor 6) which easily fits in one image window (last part graph).

Other techniques with the potential to significantly reduce acquisition time include MR-fingerprinting (31) and magnetic resonance spin tomography in timedomain (MR-STAT) (32). In both techniques, the carefully designed pulse sequences traditionally used to acquire MR images are abandoned, heavily reducing scan time. Instead of employing different sequences to measure different parameters, several parameters are measured in a single examination. Theoretically, the behavior of the MR signal for each pulse sequence can be modelled using the Bloch equations. However, doing this for each voxel at each time point requires a significant amount of computational power. In MR fingerprinting, this problem is partly circumvented by the construction of a library of signal behaviors over time for each possible combination of values of the parameters of interest. The actual measured signal can be matched to the corresponding library entry. In MR-STAT, the image is reconstructed by directly solving the Bloch equations for the signal behavior in each voxel. Currently, reconstruction takes hours for a single 2D brain slice (1x1 $\mathrm{mm}^{2}$ voxel size) on a high performance computing cluster,(33) but according to Moore's law, clinical MR-STAT should soon be within reach.(34) 


\section{Data Analysis}

A single multiparametric kidney examination as presented in this thesis generates around 1-2 GB of data - unprocessed. After processing, currently around 10 parameter maps are generated per kidney. To perform quantitative comparisons between groups of patients, numbers have to be coupled to these maps. Most commonly, region of interest-based analyses are used, where a researcher manually delineates kidney parenchyma and discriminates cortex from medulla. The mean value of those regions denotes the right medullary $\mathrm{T}_{1}$, or the perfusion of the left kidney.

This approach is far from ideal and a potential cause of burnout or RSI thanks to the numerous hours that need to be spent contouring MR images. Many algorithms exist to achieve this in a fully or semi-automated way: thresholding based; numerous classification and clustering based methods like k-means clustering as I used in chapter 2 and 3; region growing based methods and various combinations of those and other approaches, but these are far from perfect.(35) A solution seems close in the form of automated contouring by machine-learning algorithms. If enough data is available, neural networks (especially U-NET-type networks (36)) are capable of "learning" to recognize features on images, in case of the kidneys including cortex and medulla. In machine learning, the computer looks at a database of images and corresponding segmentations over and over, while continuously checking whether the predictions (kidney segmentations) improve when the neural network parameters are adjusted in a certain direction. After every few images, the parameters are adjusted. Step by step, it gets better and better in segmenting images. The one main reason that not everyone is using neural networks yet for medical image segmentation, is that it requires a significant amount of data to properly train a network. This data has not only to be acquired, but also high-quality manual segmentations have to be made for the network to train on. A network trained on anatomical $\mathrm{T}_{1}$ weighted images needs additional training to learn how to segment BOLD images. Considering this, it is only a question of time, RSI and burnouts for neural networks to become readily available for the analysis of our multiparametric kidney MRI data.

A 25 minute multiparametric MRI examination, highly accelerated thanks to compressed sensing and high density receive arrays, analyzed fully automatically within two hours after acquisition on a server directly connected to the MRI scanner, thanks to the neural network which could do the ROI selection - and we are left with an array of numbers. We had $2 \mathrm{~GB}$ of data - we reduced it to less than $1 \mathrm{~KB}$.

Reducing a series of pictures to a series of numbers - or a series of two numbers, one for cortex, one for medulla - essentially means discarding valuable information. Subtle, local changes are lost in the mean. In chapter 7 I tried to tackle this by a histogram-based analysis, which allows assessment of the distribution of values, rather than just the mean. In research however, statistical comparison of 
histograms between groups is not straightforward. And also in clinical practice, assessment of multiple histograms for each MRI might not be straightforward.

So, how should we handle the data we are generating? This is an open question and relevant in more fields than just renal imaging. It is certain that machine learning approaches will be part of the solution, but computers are only as good and unbiased as the data they are fed with and as the people writing their algorithms. For multiparametric (renal) imaging, I imagine that computers will learn to recognize patterns corresponding to common pathologies. An approach that is particularly promising in this regard is radiomics. In radiomics, images are considered a source of data from which complex features can be extracted which, ideally, are characteristic for specific pathologies. For example, it is used in research settings for subtyping renal malignancies and monitoring of treatment response.(37) In radiomics, image segmentations are required, but it might be possible to skip this step altogether and feed entire images to algorithms. And why only images? Why not add other clinically relevant information? Computing power and memory might currently be a problem, but this is probably only temporary. Another challenge might be to maintain understanding of the reasons and assumptions underlying predictions generated by such large networks - to avoid the "black box" problem. However, the network can be forced to produce interim outputs or alternatively, several networks might be linked together, which enables assessment of the interim results on which predictions are based. To train such algorithms however, data is needed - lots of data.

\section{Social and Environmental Impact}

An MRI scanner is expensive to purchase, use and maintain - both from a financial as well as an environmental perspective. The carbon footprint for a single MRI examination was calculated to be around $22.4 \mathrm{~kg} \mathrm{CO}_{2}$ equivalents $\left(\mathrm{CO}_{2} \mathrm{eq}\right)$, which is roughly the same as a $200 \mathrm{~km}$ car drive.(40) However, this number heavily depends on the utilization of the scanner. Interestingly, only $38 \%$ of emissions are caused by the actual scan.(40) The rest is caused by the energy consumption of the MR system while idle, which is considerable, since it can only partly shut down (the magnetic field cannot be turned off and therefore the cooling system has to keep running). The $22.4 \mathrm{~kg} \mathrm{CO}_{2}$ eq for one patient examination is based on a $72 \%$ utilization rate during a 12-hour shift on weekdays, but it increases about threefold for a scanner with a utility rate of $50 \%$ during an 8-hour shift. Thus, in order to save energy an MR scanner should be used as efficient as possible.

Ultimately, it is up to the vendors to reduce energy consumption of medical systems. The use of high-temperature superconducting materials might be beneficial to reduce energy consumption of the cooling system.(41) Furthermore, it might be possible to switch amplifiers off instead of using the stand-by mode. In addition, 
hospitals can contribute by critically assessing workflows regarding energy consumption and resource use.

Regarding potential uses of MRI as discussed in the first section, performing an MRI instead of a biopsy might be cost effective. Furthermore, if MRI can help in early detection of renal disease and in preventing progression to end-stage renal disease, this might be beneficial from both a financial and environmental perspective, However, there is a risk of mitigating these expenses to other departments of the healthcare system, since in the end, everyone has to die of something. ${ }^{1}$

\section{Conclusion}

This thesis covers the road from technical development to clinical validation of renal MRI. In the first part, multiparametric MRI, including challenging techniques like DCE MRI, was shown to be feasible and repeatable in kidneys. MRI is generally considered safe since patients are not exposed to radiation as in CT. In chapter 4 we report that the pharmacokinetics of gadolinium-based contrast agents in humans are not fully understood after the first phases of distribution and elimination. Although the remnant amounts detected in the kidney one week after administration are not considered harmful, further research is required to fully understand the kinetics.

The next step is to determine how the potential of renal multiparametric MRI can be fully employed for patients with kidney disease. As a diagnostic and prognostic tool, MRI may help keeping patients away from renal replacement therapies as dialysis and transplantation. In the second part of this thesis, it was shown that MRI can detect crucial physiological and structural changes in patients suffering from hypertension, where $\mathrm{R}_{2}^{*}$ correlated with activity of the renin angiotensin aldosterone system, but also in a patient suffering from end-stage failure of her renal transplant. In this patient, the findings of the multiparametric MRI examination could be correlated with histology and clinically crucial changes as interstitial fibrosis, tubular atrophy and capillary rarefaction could be successfully detected with MRI.

In conclusion, MRI can detect clinically important functional and microstructural determinants of renal health. This justifies the initiation of largescale prospective studies. Cooperation of nephrologists, radiologists and MR physicists is crucial to determine how exactly MRI can be deployed to stop further loss of kidney function in patients.

$1 \quad$ P.J. Blankestijn, personal communication, 2019 


\section{References}

1. Smith FW, Hutchison JM, Mallard JR, Reid A, Johnson G, Redpath TW, et al. Renal cyst or tumour? Differentiation by whole-body nuclear magnetic resonance imaging. Diagnostic imaging. 1981;50(2):61-5.

2. Brachemi S, Bollee G. Renal biopsy practice: What is the gold standard? World journal of nephrology. 2014;3(4):287-94.

3. Friedli I, Crowe LA, Berchtold L, Moll S, Hadaya K, de Perrot T, et al. New Magnetic Resonance Imaging Index for Renal Fibrosis Assessment: A Comparison between Diffusion-Weighted Imaging and T1 Mapping with Histological Validation. Scientific reports. 2016;6:30088.

4. Bane O, Hectors SJ, Gordic S, Kennedy P, Wagner M, Weiss A, et al. Multiparametric magnetic resonance imaging shows promising results to assess renal transplant dysfunction with fibrosis. Kidney international. 2020;97(2):414-20.

5. Wang W, Yu Y, Wen J, Zhang M, Chen J, Cheng D, et al. Combination of Functional Magnetic Resonance Imaging and Histopathologic Analysis to Evaluate Interstitial Fibrosis in Kidney Allografts. Clinical journal of the American Society of Nephrology : CJASN. 2019;14(9):1372-80.

6. Graham-Brown MP, Singh A, Wormleighton J, Brunskill NJ, McCann GP, Barratt J, et al. Association between native T1 mapping of the kidney and renal fibrosis in patients with IgA nephropathy. BMC nephrology. 2019;20(1):256.

7. Rapacchi S, Smith RX, Wang Y, Yan L, Sigalov V, Krasileva KE, et al. Towards the identification of multi-parametric quantitative MRI biomarkers in lupus nephritis. Magn Reson Imaging. 2015;33(9):1066-74.

8. Caroli A, Schneider M, Friedli I, Ljimani A, De Seigneux S, Boor P, et al. Diffusionweighted magnetic resonance imaging to assess diffuse renal pathology: a systematic review and statement paper. Nephrology, dialysis, transplantation : official publication of the European Dialysis and Transplant Association - European Renal Association. 2018;33(suppl_2):ii29-ii40.

9. Marticorena Garcia SR, Grossmann M, Bruns A, Durr M, Tzschatzsch H, Hamm B, et al. Tomoelastography Paired With $\mathrm{T}^{*}$ Magnetic Resonance Imaging Detects Lupus Nephritis With Normal Renal Function. Investigative radiology. 2019;54(2):89-97.

10. Ferreira VM, Schulz-Menger J, Holmvang G, Kramer CM, Carbone I, Sechtem U, et al. Cardiovascular Magnetic Resonance in Nonischemic Myocardial Inflammation: Expert Recommendations. Journal of the American College of Cardiology. 2018;72(24):3158-76.

11. Spieker M, Katsianos E, Gastl M, Behm P, Horn P, Jacoby C, et al. T2 mapping cardiovascular magnetic resonance identifies the presence of myocardial inflammation in patients with dilated cardiomyopathy as compared to endomyocardial biopsy. European heart journal cardiovascular Imaging. 2018;19(5):574-82.

12. Di Cesare E. MRI of the cardiomyopathies. European journal of radiology. 2001;38(3):179-84. 
13. Matsumoto S, Mori H, Miyake H, Yamada Y, Ueda S, Oga M, et al. MRI signal characteristics of progressive massive fibrosis in silicosis. Clinical radiology. 1998;53(7):510-4.

14. Pruijm M, Mendichovszky IA, Liss P, Van der Niepen P, Textor SC, Lerman LO, et al. Renal blood oxygenation level-dependent magnetic resonance imaging to measure renal tissue oxygenation: a statement paper and systematic review. Nephrology, dialysis, transplantation : official publication of the European Dialysis and Transplant Association - European Renal Association. 2018;33(suppl_2):ii22-ii8.

15. Wentland AL, Sadowski EA, Djamali A, Grist TM, Becker BN, Fain SB. Quantitative MR measures of intrarenal perfusion in the assessment of transplanted kidneys: initial experience. Academic radiology. 2009;16(9):1077-85.

16. Hueper K, Gueler F, Bräsen JH, Gutberlet M, Jang M-S, Lehner F, et al. Functional MRI detects perfusion impairment in renal allografts with delayed graft function. American Journal of Physiology-Renal Physiology. 2015;308(12):F1444-F51.

17. Hueper K, Khalifa AA, Brasen JH, Vo Chieu VD, Gutberlet M, Wintterle S, et al. Diffusion-Weighted imaging and diffusion tensor imaging detect delayed graft function and correlate with allograft fibrosis in patients early after kidney transplantation. Journal of magnetic resonance imaging : JMRI. 2016;44(1):112-21.

18. Steiger P, Barbieri S, Kruse A, Ith M, Thoeny HC. Selection for biopsy of kidney transplant patients by diffusion-weighted MRI. European radiology. 2017;27(10):4336-44.

19. Pruijm M, Milani B, Pivin E, Podhajska A, Vogt B, Stuber M, et al. Reduced cortical oxygenation predicts a progressive decline of renal function in patients with chronic kidney disease. Kidney international. 2018;93(4):932-40.

20. Pruijm M, Hofmann L, Piskunowicz M, Muller ME, Zweiacker C, Bassi I, et al. Determinants of renal tissue oxygenation as measured with BOLD-MRI in chronic kidney disease and hypertension in humans. PloS one. 2014;9(4):e95895.

21. Li X, Auerbach EJ, Van de Moortele PF, Ugurbil K, Metzger GJ. Quantitative single breath-hold renal arterial spin labeling imaging at $7 \mathrm{~T}$. Magnetic resonance in medicine. 2018;79(2):815-25.

22. Nery F, Buchanan CE, Harteveld AA, Odudu A, Bane O, Cox EF, et al. Consensus-based technical recommendations for clinical translation of renal ASL MRI. Magma (New York, NY)2019.

23. Zhang X, Petersen ET, Ghariq E, De Vis JB, Webb AG, Teeuwisse WM, et al. In vivo blood $\mathrm{T}(1)$ measurements at $1.5 \mathrm{~T}, 3 \mathrm{~T}$, and $7 \mathrm{~T}$. Magnetic resonance in medicine. 2013;70(4):1082-6.

24. Qualification of Biomarker - Total Kidney Volume in Studies for Treatment of Autosomal Dominant Polycystic Kidney Disease2 February 2020:[2 p.].

25. Dekkers IA, de Boer A, Sharma K, Cox EF, Lamb HJ, Buckley DL, et al. Consensus-based technical recommendations for clinical translation of renal T1 and T2 mapping MRI. Magma (New York, NY)2019.

26. Prasad PV, Epstein FH. Changes in renal medullary pO2 during water diuresis as evaluated by blood oxygenation level-dependent magnetic resonance imaging: effects of 
aging and cyclooxygenase inhibition. Kidney international. 1999;55(1):294-8.

27. Deprez S, de Ruiter MB, Bogaert S, Peeters R, Belderbos J, De Ruysscher D, et al. Multicenter reproducibility of structural, diffusion tensor, and resting state functional magnetic resonance imaging measures. Neuroradiology. 2018;60(6):617-34.

28. Lustig M, Donoho D, Pauly JM. Sparse MRI: The application of compressed sensing for rapid MR imaging. Magnetic resonance in medicine. 2007;58(6):1182-95.

29. Hardy CJ, Giaquinto RO, Piel JE, Rohling KW, Marinelli L, Blezek DJ, et al. 128-channel body MRI with a flexible high-density receiver-coil array. Journal of magnetic resonance imaging : JMRI. 2008;28(5):1219-25.

30. Hendriks AD, Luijten PR, Klomp DWJ, Petridou N. Potential acceleration performance of a 256-channel whole-brain receive array at $7 \mathrm{~T}$. Magnetic resonance in medicine. 2019;81(3):1659-70.

31. Ma D, Gulani V, Seiberlich N, Liu K, Sunshine JL, Duerk JL, et al. Magnetic resonance fingerprinting. Nature. 2013;495(7440):187-92.

32. Sbrizzi A, Heide OV, Cloos M, Toorn AV, Hoogduin H, Luijten PR, et al. Fast quantitative MRI as a nonlinear tomography problem. Magn Reson Imaging. 2018;46:56-63.

33. van der Heide O, Sbrizzi A, Luijten PR, van den Berg CAT. High-resolution in vivo MRSTAT using a matrix-free and parallelized reconstruction algorithm. NMR in biomedicine. 2020:e4251.

34. Moore GE. Cramming more components onto integrated circuits, Reprinted from Electronics, volume 38, number 8, April 19, 1965, pp.114 ff. IEEE Solid-State Circuits Society Newsletter. 2006;11(3):33-5.

35. Norouzi A, Rahim MSM, Altameem A, Saba T, Rad AE, Rehman A, et al. Medical Image Segmentation Methods, Algorithms, and Applications. IETE Technical Review. 2014;31(3):199-213.

36. Ronneberger O, Fischer P, Brox T, editors. U-Net: Convolutional Networks for Biomedical Image Segmentation2015; Cham: Springer International Publishing.

37. de Leon AD, Kapur P, Pedrosa I. Radiomics in Kidney Cancer: MR Imaging. Magn Reson Imaging Clin N Am. 2019;27(1):1-13.

38. Eurostat. Healthcare expenditure statistics 2019 [cited 20206 February]. Available from: https://ec.europa.eu/eurostat/statistics-explained/index.php?title=Healthcare expenditure_statistics.

39. Karlsson M, Pigretti Öhman D. Material consumption in the healthcare sector: Strategies to reduce its impact on climate change-The case of Region Scania in South Sweden. Journal of Cleaner Production. 2005;13(10):1071-81.

40. Esmaeili A, McGuire C, Overcash M, Ali K, Soltani S, Twomey J. Environmental impact reduction as a new dimension for quality measurement of healthcare services. Int $\mathrm{J}$ Health Care Qual Assur. 2018;31(8):910-22.

41. Cheong H-S, Wild J, Alford N, Valkov I, Randell C, Paley M. A high temperature superconducting imaging coil for low-field MRI. Concepts in Magnetic Resonance Part B: Magnetic Resonance Engineering. 2010;37B(2):56-64. 

Appendices

Nederlandse Samenvatting

(English) Summary

List of Publications and Presentations

Dankwoord (Acknowledgements)

Curriculum Vitae 


\section{Nederlandse Samenvatting}

Een tekort aan orgaandonoren - daar heeft iedereen wel eens van gehoord. Of van mensen die een nier doneren aan een familielid. Of misschien weet je iets van dialyse, een levensreddende behandeling voor mensen wiens nieren niet meer werken waarbij je wel drie tot vier keer per week urenlang aan een dialyseapparaat moet liggen.

Nierziektes zijn niet zeldzaam. Bij zo'n tien procent van de mensen functioneren de nieren niet optimaal. De meeste mensen merken daar niets van, want als je nieren langzaam achteruit gaan geeft dat niet of nauwelijks klachten. Het probleem is: de schade die op deze manier ontstaat is niet meer te repareren.

Daarom is het zo belangrijk om nierproblemen op tijd op te sporen. Maar hierdoor is het ook zo moeilijk om dat te doen. Zonder klachten gaat iemand immers niet naar de dokter.

Wel zijn er mensen die een grotere kans hebben op nierproblemen: bijvoorbeeld mensen met suikerziekte (diabetes) of een hoge bloeddruk. Zij komen wel regelmatig bij een arts. Maar de onderzoeken die hun arts op dit moment doet om nierschade op te sporen, geven pas in een heel laat stadium afwijkende resultaten. Bovendien zeggen ze nauwelijks iets over wát er precies mis is met de nieren. Een groeiende groep onderzoekers en nefrologen denkt dat uitgebreide MRI onderzoeken die informatie wel kunnen geven, en bovendien al in een vroeg stadium veranderingen laten zien. En dat is waar dit proefschrift over gaat.

De introductie van dit proefschrift is opgedeeld in twee delen. Het eerste deel (hoofdstuk 1.2, Nephrology for Physicists) geeft wat achtergrond over de anatomie (hoe ze in elkaar zitten) en de fysiologie (hoe ze werken) van de nieren. Het tweede deel (hoofdstuk 1.3, MR Physics for Physicians) is een introductie over de natuurkunde achter een MRI scanner.

In hoofdstuk 2 wordt een uitgebreid MRI protocol beschreven wat we tijdens mijn promotie samengesteld en ontwikkeld hebben. Dat werd getest op reproduceerbaarheid: als je twee keer hetzelfde meet, komt er dan ook twee keer hetzelfde uit? Om dat te testen werden negentien gezonde vrijwilligers twee keer gescand met ongeveer een week ertussen, en werden de resultaten met elkaar vergeleken. 
In het MRI protocol zitten verschillende scans. Zo meten we de zuurstofvoorziening met een techniek die BOLD (Blood Oxygenation Level Dependent) MRI heet. Er zijn verschillende technieken waarmee de doorbloeding gemeten wordt: ASL (Arterial Spin Labeling) en DCE (Dynamic Contrast Enhanced). Het voordeel van ASL is dat er geen contrastmiddel toegediend hoeft te worden via een infuus. Bovendien bleken de metingen met ASL reproduceerbaarder. Het voordeel van DCE is dat je daarmee een heel belangrijke functie van de nier kunt meten: de snelheid waarmee het bloed gefilterd wordt oftewel de GFR. Terwijl ASL en DCE de doorbloeding van de nier in kaart brengen, kun je met weer een andere techniek meten hoeveel bloed er in totaal door de nierslagader stroomt.

Ook voor het in kaart brengen van de structuur van het nierweefsel werden verschillende technieken gebruikt. Met DWI (Diffusion Weighted Imaging) kun je kijken hoe watermoleculen zich precies door de nieren bewegen. Door bindweefsel, maar ook door de aanwezigheid van ontstekingscellen, kunnen die minder vrij bewegen. Tot slot meten we met $\mathrm{T}_{1}$ en $\mathrm{T}_{2}$ mapping magnetische eigenschappen van het weefsel, die snel veranderen bij nierschade.

Uit de resultaten bleek dat de meeste metingen ongeveer even reproduceerbaar waren als veelgebruikte klinische onderzoeken.

In hoofdstuk 3 wordt op één specifieke techniek ingegaan: DCE MRI. Bij DCE MRI worden gedurende meer dan 5 minuten continu snelle beelden van de nieren gemaakt, terwijl via een infuus contrastmiddel wordt gegeven. Hierdoor veranderen de nieren op de MRI beelden van kleur. Uit die kleurverandering (contrastverandering) kunnen de doorbloeding en filtreersnelheid (GFR) gemeten worden. Het probleem is dat de nieren meebewegen op de ademhaling. En doordat ze van kleur veranderen is dat moeilijk te corrigeren met behulp van automatische algoritmes. Wij stellen voor om net iets andere beelden te verzamelen, namelijk zogenaamde "vet-" en "waterbeelden", waar respectievelijk vet en water een hoge intensiteit hebben. Omdat het contrastmiddel het vetweefsel niet bereikt, is de kleur van het vetbeeld constant. Daardoor kan het vetbeeld gebruikt worden voor de bewegingscorrectie, en dat bleek inderdaad goed te werken.

Bij de reproduceerbaarheidsstudie (hoofdstuk 2) merkten we iets vreemds: de $\mathrm{T}_{1}$ waarde van de nieren bleek de tweede keer structureel lager te zijn dan de eerste keer, terwijl we twee keer precies hetzelfde gedaan hadden. Na onze hersens gepijnigd te hebben, kwamen we tot de conclusie dat het contrastmiddel wat de deelnemers kregen waarschijnlijk langer in het lichaam bleef dan we dachten - dat zorgt namelijk voor 
een daling in de $\mathrm{T}_{1}$. In hoofdstuk 4 proberen we dit te bevestigen. Zo controleerden we of het verschil in $\mathrm{T}_{1}$ samenhing met de tijd verstreken sinds de eerste scan (en de toediening van het contrastmiddel). Dat was zo. Vervolgens keken we of het $\mathrm{T}_{1}$ verschil samenhing met de nierfunctie, want je zou verwachten dat mensen met een betere nierfunctie het contrastmiddel sneller uitscheiden en daardoor een kleiner verschil in $\mathrm{T}_{1}$ hebben. Dat was ook zo. Dit zou betekenen dat het laatste beetje contrastmiddel in het lichaam veel trager uitgescheiden wordt dan gedacht. Dat is niet geheel onverwacht, want er zijn verschillende studies geweest die laten zien dat contrastmiddel na herhaaldelijk toedienen nog jaren later aantoonbaar is in brein, botten en huid. Onze studie bevestigt nog maar eens dat hier verder onderzoek naar gedaan moet worden.

Hoofdstuk 5 en 6 beschrijven twee onderzoeken naar de zuurstofvoorziening van de nieren bij patiënten met een te hoge bloeddruk. Artsen denken dat een hoge bloeddruk zorgt voor een zuurstoftekort in de nieren, maar dat dóór dat zuurstoftekort de bloeddruk ook weer omhoog gaat. Een vicieuze cirkel dus. Daarom dachten we dat een daling in de bloeddruk voor een betere zuurstofvoorziening zou zorgen, maar dat werd in hoofdstuk 5 niet gevonden. Wel vonden we in hoofdstuk 6 een relatie tussen de zuurstofvoorziening van de nieren en de activiteit van een belangrijk hormoonsysteem dat de bloeddruk in het lichaam reguleert.

In hoofdstuk 7 kijken we specifiek naar één patiënt met een transplantatienier. Deze nier moest verwijderd worden vanwege bloedplassen en pijnklachten. De patiënte ging akkoord met een uitgebreide MRI scan (zoals beschreven in hoofdstuk 2) vóór de operatie. Dit gaf ons de kans om de resultaten van de MRI te vergelijken met de het microscopische onderzoek van de hele nier, dat na de operatie gedaan werd. De microscopie en de resultaten van de MRI bleken heel goed met elkaar te kloppen. Zo kon het verdwijnen van de klein vaatjes met ASL MRI in beeld gebracht worden. En ook de DWI, $\mathrm{T}_{1}$ mapping en $\mathrm{T}_{2}$ mapping gaven sterk afwijkende resultaten die pasten bij de ontstekingsreactie en verbindweefseling die met de microscoop gezien werd. Het detecteren van bindweefsel en ontstekingsreacties is heel belangrijk om een behandelplan te kunnen maken, maar ook om de patiënt een betrouwbare prognose te kunnen geven. Het is dus veelbelovend dat de MRI deze veranderingen inderdaad kan detecteren.

Tot slot wordt in hoofdstuk 8 een uitstapje gemaakt naar MRI van de nieren op een speciale MRI scanner met een zeer hoog magneetveld (7 Tesla). Bij normale klinische onderzoeken wordt een veldsterkte van 1.5 of 3 Tesla gebruikt. Het verhogen van het magnetisch veld van de MRI scanner naar 7 Tesla, geeft nieuwe mogelijkheden, 
maar creëert ook nieuwe uitdagingen. In dit overzichtsartikel beschrijven we welke technieken waarschijnlijk beter werken op 7 Tesla, wat de uitdagingen zijn en wat mogelijke oplossingen zijn.

Tenslotte proberen we in hoofdstuk 9 de resultaten van deze thesis in een bredere (maatschappelijke) context te plaatsen. Zo kijken we hoe MRI van de nieren in het ziekenhuis gebruikt kan worden om die patiënten met schade aan hun nieren zo vroeg mogelijk op te sporen, zodat we verdere achteruitgang kunnen voorkomen. Daarnaast worden een aantal technische ontwikkelingen beschreven, waarmee de duur van de MRI (nu een uur) teruggebracht kan worden tot een half uur, wat goedkoper is en ook logistiek haalbaarder. Tot slot kijken we of en hoe een techniek als MRI, die gepaard gaat met zowel een significante milieubelasting als behoorlijke kosten, op een duurzame manier ingezet kan worden in een tijd waarin de noodzaak tot verduurzaming en kostenbeheersing ook doorgedrongen is tot de gezondheidszorg. 


\section{Summary}

Around $10 \%$ of the general population suffers from some form of chronic kidney damage. Yet, treatment options for chronic kidney diseases are limited. Current therapies can only slow down progression of chronic kidney disease. Early detection therefore is crucial to initiate treatment in an early stage and to prevent progression to end-stage renal disease. Existing diagnostic tests, like analysis of blood plasma and urine, only detect damage in a later stage and do not provide insight in the underlying structural and functional problems. In this thesis, we will show that MRI potentially addresses those problems and can proof to be a valuable diagnostic tool for nephrologists.

Currently application of renal MRI is limited to imaging of the renal arteries, malignancies or in case of a contra-indication for CT. We will show that advanced MR techniques provide information on both function and microstructure of the kidneys. The thesis covers the road from technical development to clinical application of multiparametric renal MRI.

The introduction is split in two parts: for readers with a background in medical imaging the section on nephrology (Chapter 1.2, Nephrology for Physicists) is probably interesting, while chapter 1.3, Physics for Physicians, is meant as an introduction to MR physics for medical doctors.

In chapter 2 a multiparametric MRI protocol designed specifically for renal imaging is tested in terms of repeatability. Nineteen healthy volunteers are scanned twice with an interval of about a week. The results of both examinations are compared. In the multiparametric MRI examination multiple techniques are combined, yielding a comprehensive image of renal microstructure and function. To obtain a measure for oxygenation, blood oxygenation level-dependent (BOLD) MRI is used. Renal perfusion is measured using both arterial spin labeling (ASL) and dynamic contrast enhanced (DCE) MRI. Contrarily to DCE, ASL does not require administration of exogenous contrast agent. In chapter 2, we show that measurement of perfusion using ASL is more reliable compared to DCE. However, with DCE the glomerular filtration rate can be measured per kidney, which is not possible with ASL. Blood flow through the renal artery is measured using 2D PC (two-dimensional phase contrast) MRI. Furthermore, diffusion weighted imaging is used to quantify and map the diffusion of water molecules. This diffusion is restricted in inflammatory conditions but also in fibrotic tissues. Finally, $\mathrm{T}_{1}$ and $\mathrm{T}_{2}$ are measured, magnetic properties of tissue sensitive to changes in microstructure, like fibrosis or edema. Based on the 
results of this study, we can conclude that repeatability of most measurements is comparable to clinical tests for renal function like eGFR and inulin clearance.

In chapter 3 an important problem in renal imaging is addressed: respiratory motion. In DCE MRI, for several minutes quick images of the kidney are continuously acquired, but due to respiratory motion those images are misaligned. Furthermore, renal contrast differs heavily between images due to inflow of contrast agent during the acquisition. This complicates re-alignment of the images using automated registration algorithms. We propose to acquire slightly different images using the Dixon technique: "water" and "fat" images with high intensity of water and fat, respectively. Contrast agent does not reach adipose tissue, so the contrast in fat images remains constant. Therefore, the fat images can be used for motion correction. In chapter 3 , we show that the use of fat images for image registration indeed outperforms the conventional approach. Subsequently, for the DCE analysis water images are used.

Chapter 4 describes an incidental finding in the repeatability study (chapter 3). Renal $\mathrm{T}_{1}$ was significantly lower in the second measurements compared to the first. We hypothesized that this bias was caused by delayed excretion of the contrast agent, which was administered during the first scan. Indeed, the $\mathrm{T}_{1}$ bias was not seen in subjects who did not receive contrast agent. Furthermore, the $T_{1}$ difference correlated with both renal function as well as time in between scans (e.i. time since administration). The latter suggests that a week after administration still contrast agent is being excreted. In the summary of product characteristics of the contrast agent however, de half-life of is given as 1.8 hour. This implies that after a week, only an undetectable fraction of $10^{-28}$ of the original dose remains. Delayed excretion of contrast agents was previously only described in animal studies, usually carried out with relative dosages orders of magnitude higher than dosages used in humans. This study should be seen in the light of other studies which showed retention of MR contrast agents in brain, bones and skin. The current study once again shows that the mechanism of excretion of MR contrast agents is not fully understood. Further research is therefore indicated.

In chapter 5 and 6 BOLD MRI is used to assess renal oxygenation in patients with hypertension. Hypertension can cause renal damage, but renal disease is almost always accompanied by hypertension. The hypothesis is that hypertension causes renal hypoxia, which in turn increases blood pressure via feedback mechanisms. Therefore, one would think that a decrease in blood pressure leads to improved oxygenation. However, in chapter 5 this could not be demonstrated. Nevertheless, 
in chapter 6 a relation was found between activity of the renin-angiotensinaldosterone system and renal oxygenation. This system plays an important role in blood pressure regulation, and increased activity increases blood pressure. Indeed renal hypoxia as measured by BOLD MRI increased with increasing activity of the renin-angiotensin-aldosterone system.

Chapter 7 describes a case of a patient with end-stage failure of her transplant kidney. The transplant kidney had to be explanted due to hematuria and persistent pain in the region of the allograft. This patient agreed to undergo a multiparametric MRI as described in chapter 2, a few days before the explantation surgery. Only DCE MRI was not carried out since administration of contrast-agent is contra-indicated in patients with severe renal failure. Since the transplant kidney was explanted, we were able to compare whole-kidney histology with the results of the multiparametric MRI. The MR measures correlated well with histological findings. For example, the disappearance of microvasculature (capillary rarefaction) could be visualized with ASL MRI. The presence of inflammation, interstitial fibrosis and tubular atrophy caused changes in the diffusivity, $\mathrm{T}_{1}$ and $\mathrm{T}_{2}$. Detection of those entities is crucial in treatment planning and prognosis, so the ability of MRI to detect this is promising.

Chapter $\boldsymbol{8}$ explores the possibilities of renal MRI at ultra-high field. Switching from a magnetic field strength of 1.5 or 3 Tesla (T), as clinically used, to 7 T creates new possibilities, but imposes additional challenges as well. This review article describes which techniques are expected to profit from 7 T MRI, what the challenges are and where to look for solutions.

Finally, chapter 9 places these results in context. How should renal MRI be used in clinical practice to aid patients and doctors in early detection of renal damage and prevention of progression to end-stage renal disease? New technical developments can bridge the gap between research and clinical use. And we will touch upon the question how to employ MRI, a technique associated with both significant environmental as well as financial costs, in a sustainable way. 


\section{List of Publications and Presentations}

\section{Peer Reviewed Articles}

First Author Only

de Boer A,* Pieters TT,* Harteveld AA, Bos C, Froeling M, Goldschmeding R, Hoogduin JM, Joles JA, Petri BJ, Verhaar MC, Leiner T, Nguyen TQ, van Zuilen AD. Multiparametric MRI evaluation of end-stage kidney disease in a kidney transplant recipient and validation by histopathology after nephrectomy. *: these authors contributed equally. MAGMA. 2020 Sep. doi: 10.1007/s10334-020-00887-9. Online ahead of print.

de Boer A, Harteveld AA, Stemkens B, Blankestijn PJ, Bos C, Franklin SL, Froeling M, Joles JA, Verhaar MC, van den Berg CAT, Hoogduin JM, Leiner T. Multiparametric Renal MRI: An Intrasubject Test-Retest Repeatability Study. J Magn Reson Imaging. 2020 Apr. doi: 10.1002/jmri.27167. Online ahead of print.

de Boer A, Harteveld AA, Pieters T, Blankestijn PJ, Bos C, Froeling M, Joles JA, Verhaar MC, Leiner T, Hoogduin JM. Decreased native renal T1 up to one week after gadobutrol administration in healthy volunteers. J Magn Reson Imaging. 2020 Aug;52(2):622-631. doi: 10.1002/jmri.27014.

de Boer A, Leiner T, Vink EE, Blankestijn PJ, van den Berg CAT. Modified Dixon based renal dynamic contrast-enhanced MRI facilitates automated registration and perfusion analysis. Magn Reson Med. 2018 Jul;80(1):66-76.

de Boer A, Hoogduin JM, Blankestijn PJ, Li X, Luijten PR, Metzger GJ, Raaijmakers AJ, Umutlu L, Visser F, Leiner T. 7 T renal MRI: challenges and promises. MAGMA. 2016 Jun;29(3):417-33.

In Preparation

de Boer A, Villa G, Bane O, Bock M, Cox EF, Dekkers IA, Eckerbom P, FernándezSeara MA, Francis ST, Haddock B, Hall ME, Hall Barrientos P, Hermann I, Hockings PD, Lamb HJ, Laustsen C, Lim RP, Morris DM, Ringgaard S, Serai SD, Sharma K, Sourbron S, Takehara Y, Wentland AL, Wolf M, Zöllner FG, Nery F, Caroli A. Consensus-based technical recommendations for clinical translation of renal phase contrast MRI. Accepted for publication in J Magn Reson Imaging. 


\section{Other}

Mendichovszky I, Pullens P, Dekkers IA, MSc; Nery F, Bane O, Pohlmann A, de Boer A, Ljimani A, Odudu A, Buchanan C, Sharma K, Laustsen C, Harteveld AA, Golay X, Pedrosa I, Alsop D, Fain S, Caroli A, Prasad PV, Francis S, Sigmund E, Seara-Fernandez M, Sourbron S. Technical recommendations for clinical translation of renal MRI: a consensus project of the Cooperation in Science and Technology Action PARENCHIMA. MAGMA. 2020 Feb;33(1):163-176. doi: 10.1007/s10334019-00784-w.

Dekkers IA, de Boer A, Sharma K; Cox E, Lamb H, Buckley DL; Bane O, Morris DM, Prasad P, Semple SIK, Gillis KA, Hockings P, Buchanan C, Wolf M; Laustsen C, Leiner T, Haddock B, Hoogduin JM, Pullens P., Sourbron S, Francis S. Consensusbased technical recommendations for clinical translation of renal T1 and T2 mapping MRI. MAGMA. 2020 Feb;33(1):163-176. doi: 10.1007/s10334-019-00797-5.

Harteveld AA, de Boer A, Franklin SL, Leiner T, van Stralen M, Bos C. Comparison of multi-delay FAIR and pCASL labeling approaches for renal perfusion quantification at 3T MRI. MAGMA. 2020 Feb;33(1):81-94. doi:10.1007/s10334-019-0o806-7.

Spit KA, Muskiet MHA, Tonneijck L, Smits MM, Kramer MHH, Joles JA, de Boer A, van Raalte DH. Renal sinus fat and renal hemodynamics: a cross-sectional analysis. MAGMA. 2020 Feb;33(1):73-80. doi: 10.1007/s10334-019-00773-z.

Wolf M, de Boer A, Sharma K, Boor P, Leiner T, Sunder-Plassmann G, Moser E, Caroli A, Jerome NP. Magnetic resonance imaging T1- and T2-mapping to assess renal structure and function: a systematic review and statement paper. Nephrol Dial Transplant. 2018 Sep 1;33(suppl_2):ii41-ii5o.

van Eijs MJM, van Zuilen AD, de Boer A, Froeling M, Nguyen TQ, Joles JA, Leiner T, Verhaar MC. Innovative Perspective: Gadolinium-Free Magnetic Resonance Imaging in Long-Term Follow-Up after Kidney Transplantation. Front Physiol. 2017 May;8:296.

Vink EE, de Boer A, Verloop WL, Spiering W, Voskuil M, Vonken E, Hoogduin JM, Leiner T, Bots ML, Blankestijn PJ. The effect of renal denervation on kidney oxygenation as determined by BOLD MRI in patients with hypertension. Eur Radiol. 2015 Jul;25(7):1984-92. 
Vink EE, de Boer A, Hoogduin HJ, Voskuil M, Leiner T, Bots ML, Joles JA, Blankestijn PJ. Renal BOLD-MRI relates to kidney function and activity of the renin-angiotensin-aldosterone system in hypertensive patients. J Hypertens. 2015 Mar;33(3):597-603.

\section{Conference Presentations}

\section{Oral Presentations}

de Boer A, "Renal MRI to detect tissue perfusion, oxygenation, inflammation and fibrosis. From technology to clinic"

- Invited presentation at the $57^{\text {th }}$ meeting of the ERA-EDTA congress, Milan, Italy, June 2020

de Boer A, "Vascular imaging - the renal arteries"

- Invited presentation at the $2^{\text {nd }}$ meeting on functional renal imaging, Nottingham, United Kingdom, 2019.

de Boer A, Harteveld AA, Blankestijn PJ, Bos C, Froeling M, Joles JA, Verhaar MC, Hoogduin JM, Leiner T. Decreased native renal $\mathrm{T}_{1}$ one week after gadobutrol administration in healthy volunteers.

- Oral presentation. In: Proceedings of the $36^{\text {th }}$ Annual Scientific Meeting of European Society of Magnetic Resonance in Medicine, Rotterdam, The Netherlands, 2019.

- Combined Oral and Poster presentation. In: Proceedings of the $2^{\text {nd }}$ meeting on functional renal imaging, Nottingham, United Kingdom, 2019. Granted a poster price.

de Boer A, Harteveld AA, Blankestijn PJ, Bos, C, Franklin SL, Froeling M, Joles JA, Verhaar MC, Hoogduin JM, Leiner T. Renal multi-parametric MRI: Ready to launch? A reproducibility study.

- Oral presentation. In: Proceedings of the $27^{\text {th }}$ Annual Meeting of ISMRM, Montreal, Canada, 2019. p. 3866.

- Oral presentation. In: Proceedings of the 11th annual meeting of ISMRM Benelux chapter, Leiden, The Netherlands, 2019.

- Combined oral and poster presentation. In: Proceedings of the Nederlandse Nefrologiedagen, Veldhoven, The Netherlands, 2019. 
de Boer A, Sanders MF, Hoogduin JM, Blankestijn PJ, Leiner T. Repeatability of renal BOLD MRI.

- Combined oral and poster presentation. In: Proceedings of the $2^{\text {nd }}$ meeting on functional renal imaging: Where Physiology, Nephrology, Radiology and Physics Meet, Berlin, Germany, 2017. p. 11

de Boer A, van den Berg CAT, Leiner T, Hoogduin JM. Semi-automated kidney delineation on BOLD images using k-means clustering of $\mathrm{R}_{2}{ }^{*}$ signal decay.

- Combined oral and poster presentation. In: Proceedings of the $2^{\text {nd }}$ meeting on functional renal imaging: Where Physiology, Nephrology, Radiology and Physics Meet, Berlin, Germany, 2017. p. 10

de Boer A, Leiner Tnd van den Berg CAT. Automated renal motion correction using fat-images derived from Dixon reconstruction of DCE MRI.

- Oral presentation. In: Proceedings of the $25^{\text {th }}$ Annual Meeting of ISMRM, Honolulu, Hawaii, USA, 2017. p. 2266. Granted a Magna Cum Laude Merit Award.

\section{Poster Presentations}

de Boer A, Sanders MF, van den Berg CAT, Blankestijn PJ, and Leiner T. Renal perfusion is decreased in kidneys with multiple renal arteries as demonstrated by renal DCE MRI.

- Poster presentation. In: Proceedings of the $25^{\text {th }}$ Annual Meeting of ISMRM, Honolulu, Hawaii, USA, 2017. p. 6600

de Boer A, Vink EE, Leiner T, Blankestijn PJ, and Hoogduin JM. The effect of renal denervation on renal oxygenation as measured on BOLD MRI.

- $\quad$ Electronic poster presentation. In: Proceedings of the $22^{\text {th }}$ Annual Meeting of ISMRM, Milan, Italy, 2014. p. 3473 


\section{Dankwoord (Acknowledgements)}

Tim, professor Leiner, voor mijn gevoel een hele tijd geleden bood je me een promotieplek aan. Geen idee of daar toen geld voor was, en dat is misschien ook wel typerend voor jou: je ziet geen obstakels, alleen mogelijkheden. Heel erg bedankt voor je enthousiaste steun!

Marianne, professor Verhaar, op de achtergrond was je altijd nauw betrokken. Ik heb ontzettend bewondering voor jouw kalme, vriendelijke en doordachte manier van leiding geven. Bedankt dat je mijn promotor wilde zijn, voor je steun en voor je betrokkenheid bij meer dan alleen mijn onderzoek.

Hans, verfrissend eerlijk en heerlijk recht voor zijn raap - meester in doorvragen tot de meest onnozel lijkende details, om er achter te komen dat dat ene detail toch niet zo onnozel was - je gaat er helemaal voor. We hebben heel wat uren doorgebracht in jouw kamer of achter de scanner, waarna ik het meestal pas echt snapte. Als het erop aankwam stond je altijd voor me klaar, bedankt!

Peter - ik kan me nog goed herinneren dat ik in de bibliotheek nefrologie zat met jou en Eva om te solliciteren op die stage over de MRI analyse van het renale denervatie project, al voelt het echt heel lang geleden. Bij jou kon ik altijd even langslopen voor goed doordacht advies, of het nou gaat over een beursaanvraag, sustainability of een ANIOS cardiologie. Dank je wel!

Ook wil ik graag de leden van de lees- en beoordelingscommissie van harte bedanken voor de tijd en moeite die jullie gestoken hebben in het beoordelen van dit proefschrift. Professor Sourbron, Steven, bedankt voor de kans om naar Engeland te komen. Het ging niet altijd van een leien dakje (of is dat een Nederlandse uitdrukking?), maar ik ben ontzettend benieuwd wat ons model op gaat leveren met de data van de Rockies studie.

Jaap, officieel was je geen copromotor maar het voelt wel zo. Als ik jou een mailtje stuurde heb ik altijd kunnen rekenen op een vrijwel instantaan antwoord. Het schijnt dat je met pensioen bent? Je zit altijd vol ideeën en ik dank nogal wat aan jouw goede oog voor mogelijke samenwerkingsverbanden. Dank je wel!

Clemens, hetzelfde geldt voor jou. Vanaf het begin was je nauw betrokken en dat is zo gebleven. Tijdens AMRP en ook daarbuiten heb je me ontzettend veel geleerd over MRI en was het ook nog eens ontzettend gezellig. Bedankt daarvoor! 
Anita en Tobias, ACRADYS buddies: als een zeker coronavirus er niet tussen was gekomen hadden we het voor elkaar gekregen om iedereen te includeren. Aan onze samenwerking heeft het in ieder geval niet gelegen. Hebben we niet nog een samenwerking-bezegelings-etentje tegoed?

Alex, Sander, Vitaliy: I honestly think our room was the "gezelligste" in the hallway. Alex, thanks for the talks about life and research, and by the way, you owe me some nuts. Sander, je vele pogingen iets van interesse bij bij me te wekken voor voetbal, NBA en wielrennen zijn helaas jammerlijk mislukt. Onze promotietrajecten begonnen ongeveer tegelijk en nu zijn we ongeveer tegelijkertijd klaar - het was heel fijn om een kamergenootje te hebben om alle onderzoeksfrustraties en -hoogtepunten (vooral frustraties) mee te delen.

Martijn: bij jou was ik altijd welkom voor elke vraag en een kort antwoord bestond niet. Heel erg bedankt voor alle hulp en natuurlijk de onvergetelijke vrimibo's!

Edwin, Jacob-Jan, Kees, Lieke, Carlo, Evita, Jeanine, Zhara, Ayhan, Kyung-Min, Seb: zonder jullie was er geen koffietrein geweest, en dat was toch wel dé reden om enigszins op tijd op werk te verschijnen (je zou hem maar mislopen).

Dennis (professor Klomp), Janot, Oscar, Peter, Arjen, Tim, Catalina, Jannie, Wouter, Jeroen, Tijl, Erwin, Bart, Carel, Sara, Lisa, Tine, Mario, Kyung-Min, Seb, Rick, Sarah, Ria, Quincy, Lennart, Anna, Carrie, Fredy, Natalia, Jaco, Wiebe, Sylvia, Mike, Mike, Hannah, Niek, Ayhan, iedereen van het coil-lab en iedereen van de $7 \mathrm{~T}$ groep die ik nu onvermijdelijk vergeet... Zonder jullie zou de $7 \mathrm{~T}$ groep de $7 \mathrm{~T}$ groep niet zijn, wat natuurlijk redelijk voor zich spreekt, maar waarmee ik dus eigenlijk wil zeggen dat de $7 \mathrm{~T}$ groep volgens mij best wel bijzonder is. Dankzij jullie waren de afgelopen jaren onvergetelijk gezellig, leerzaam, stimulerend en vooral gewoon heel erg leuk. Bedankt!

Eva, Margreet, Rosa, jullie hebben me wegwijs gemaakt bij de nefrologie, de renale denervatie, en klinisch onderzoek in het algemeen - bedankt daarvoor!

En laat ik vooral ook niet vergeten de mensen van de radiotherapie en de Drag 'n Drop ASL-groep te bedanken voor hun samenwerking, voorzover ik dat nog niet gedaan heb: Nico, Bjorn, Matteo, Bjorn, Nico, Tom, Ellis, Marijn, Isabell, Suzanne (en Clemens en Anita horen hier ook weer bij natuurlijk).

Hanneke, Eelco, Jasmijn, Wouter, Suze, Mimount, Rob, Nicola, Zonne, Willemiek, 
Floris, Maartje, Marijke, Arjan: van rolstoelvoetbal tot intervisie - we hebben hoogtepunten en dieptepunten met elkaar gedeeld. Hanneke, we hebben die website toch maar mooi voor elkaar gekregen, al heeft de Suerman het dan niet overleefd... En Lisan, bedankt voor alles, voor de geweldige masterclasses, de intervisie, en dat je deur altijd open stond. Ik heb er ontzettend van genoten en heel veel van geleerd!

Carin en Arda: zonderjullie was het niets geworden. Bedankt voor alleorganisatorische en logistieke steun, en alle moeite die jullie je getroost hebben om Tim en Marianne telkens weer op dezelfde tijd in dezelfde kamer te krijgen.

Arjan van Zuilen, Maarten Rookmaker, Daniël van Raalte, Anne Hesp (en eigenlijk horen Marianne Verhaar, Peter Blankestijn en Tobias Pieters ook weer in dit rijtje): jullie gaven een extra dimensie aan mijn promotietraject. Alles wat ik bij de divisie Beeld op MRI-vlak heb geleerd kon ik dankzij jullie meteen toepassen. Heel erg bedankt daarvoor, jullie hebben deze thesis naar een hoger vlak getild.

Kanishka, Claudio, Graham, Guilhem, Fung, Adriana, Samer, Jimmy, Nick, Jim and everyone I am inevitably forgetting from Polaris: thanks for welcoming me in your group and letting me join for Indian food, climbing, poker, gigs and scanning!

Anna, Giulia, thank you for the invitation to join in the recommendation project. It has been, and still is, a honour and pleasure working with you.

Peter-Paul, Marloes, Dominique, Lucy, Simone, Robert, Eke, Dianne, Marloes, Romy, Paul, Hind, Michiel, Lena, bedankt voor het warme welkom in de assistentengroep. Alle cardiologen in het JBZ, bedankt dat ik bij jullie weer wegwijs mocht worden in de kliniek en voor de kennismaking met jullie mooie vak.

Ilya, Stephanie, Jolien - we zien elkaar veel te weinig maar dat maakt niet uit, want als we elkaar zien is het altijd weer als vanouds.

Hans, Netty, Thomas: natuurlijk moest iemand als Pim uit zo'n warm nest komen. $\mathrm{Na}$ meer dan tien jaar voelen jullie als een tweede familie.

Pa, Ma, Maartje, Josine, Steven, Iris, Noortje - een paar maandjes Sheffield hebben me nog wat meer doen realiseren wat jullie voor me betekenen. Ma, ons uitje in the peaks was onvergetelijk, ik zie je nog die rotsen op klauteren in de kou. Jij hebt me geleerd dat ik me moet blijven afvragen wáárom iemand doet wat hij doet, en daar heb ik nog elke dag baat bij. Pa, je mag dan altijd zoek zijn, je bent er ook altijd om 
op terug te vallen, niet alleen voor mij, maar ook voor Margreet en voor Josine en Maartje en je ouders en je kleindochters. Maartje, bedankt dat je me er aan blijft herinneren dat er meer is dan werk, hoe leuk ook. Ik denk dat ik eindelijk door heb dat je gelijk hebt. Josine en Steven, Iris en Nora, zo af en toe waaien we eens bij elkaar aan voor koffie of eten, een fietstochtje misschien - het was eigenlijk toeval dat we (bijna) in dezelfde plaats belandden, maar dankzij jullie wil ik eigenlijk niet weg. Dan maar nog iets dichterbij in een kolengestookt boerderijtje.

Pim, ik vraag me soms af waar ik jou aan verdiend heb. Je bent oprecht de meest vriendelijke, geduldige, integere en betrouwbare man die ik ooit ontmoet heb. Met jou naast me zou ik haast in god gaan geloven. En dan heb je ook nog eens een geweldig gevoel voor humor en design. ${ }^{1}$ Bedankt dat je er bent.

$1 \quad$ Zie omslag 


\section{Curriculum Vitae}

Anneloes de Boer was born in 1990 as the third daughter of an engineer and a pedagogue. After finishing high-school cum laude in 2008 she moved to Enschede to study Applied Physics, driven by the desire to learn something really complicated. She extremely enjoyed the mathematical puzzling to construct complex models describing physical phenomena. However, after some years she realized that she merely was learning to describe things in a sufficiently accurate but often rather simplified way. And, rather unforeseen, she missed the actual

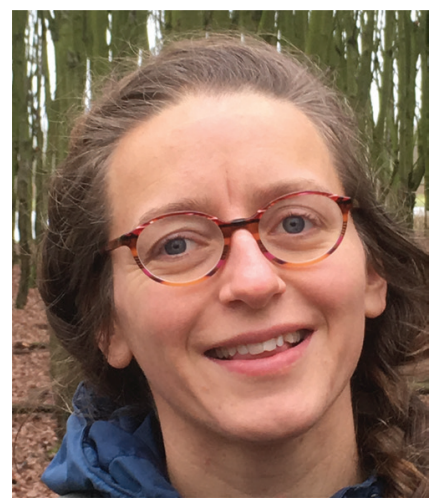
learning, the studying and gathering of new insights in topics broader than just physics. Since medicine always seemed quite attractive to her as a profession, she switched to Medicine at Utrecht University in 2010. She was able to finish her bachelor's Medicine in 2013, in parallel to her bachelor's degree in Applied Physics in 2012.

During med school she started to miss the puzzles. Therefore, she applied for the Honours program and started some research at the nephrology department alongside her Medicine study. In the analysis of comprehensive renal MRI data, her physics background was of great use. An Alexandre Suerman personal stipend allowed her to continue her research on renal MRI as part of an MD-PhD project after her graduation in 2016. During her PhD, she was supervised by prof. Tim Leiner, prof. Marianne Verhaar, dr. Hans Hoogduin and dr. Peter Blankestijn.

In her spare time she likes to play her violin, a childhood passion which never faded. In summertime, she loves sailing and she inherited a strong and irrational preference for wooden dinghies. During winters, she prays for frost and ice but is usually banished to indoor ice-rinks to at least maintain her skating skills. Last year, she discovered yoga as an enjoyable and efficient way to retain some range of motion in her neck and shoulders and started horse riding lessons.

Currently (October/November 2020), Anneloes is working at the Jeroen Bosch Hospital at the internal medicine department. 



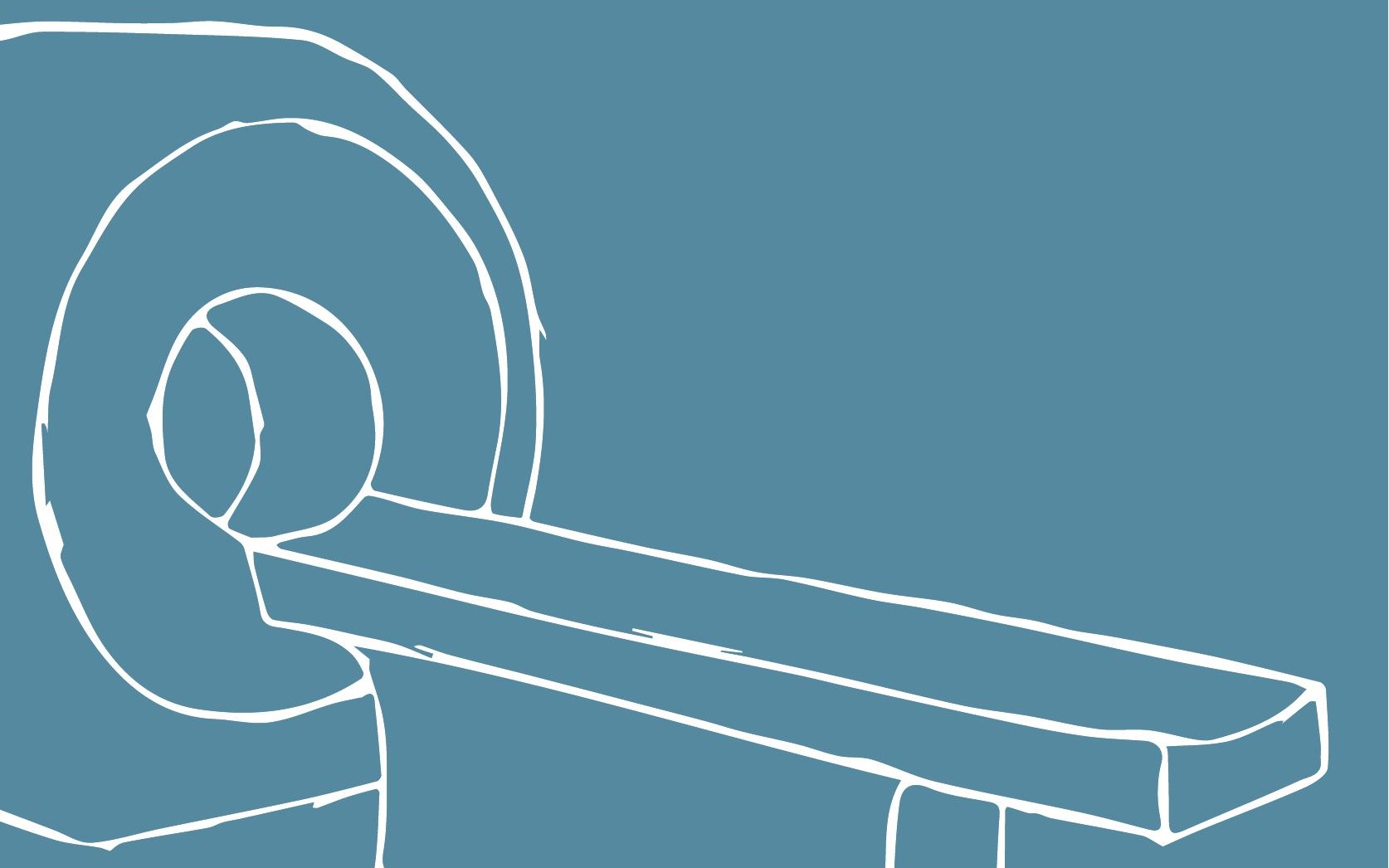

